Supporting Information

\title{
"Clickable" albumin binders for modulating the tumor uptake of targeted radiopharmaceuticals
}

Florian Brandta,b, Martin Ullrich ${ }^{\mathrm{a}}$, Markus Laube ${ }^{\mathrm{a}}$, Klaus Kopka ${ }^{\mathrm{a}, \mathrm{b}}$, Michael Bachmann ${ }^{\mathrm{a}, \mathrm{c}}$, Reik Löser ${ }^{a, b}$, Jens Pietzscha,b, Hans-Jürgen Pietzscha,b, Jörg van den Hoffa,d, Robert Wodtke ${ }^{a,{ }^{*}}$

[a] Helmholtz-Zentrum Dresden-Rossendorf, Institute of Radiopharmaceutical Cancer Research, Bautzner Landstraße 400, 01328 Dresden, Germany

[b] Technische Universität Dresden, Faculty of Chemistry and Food Chemistry, Mommsenstraße 4, 01069 Dresden, Germany

[c] National Center for Tumor Diseases (NCT) Dresden, University Hospital Carl Gustav Carus, Fetscherstraße 74, Dresden 01307, Germany

[d] Technische Universität Dresden, Department of Nuclear Medicine, University Hospital Carl Gustav Carus, Fetscherstraße 74, Dresden 01307, Germany

*E-mail: r.wodtke@hzdr.de 


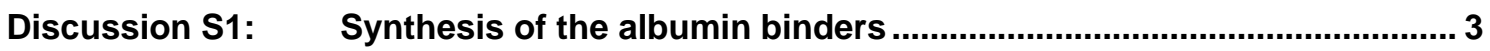

Discussion S2: $\quad$ Prediction of $K_{d}$ values for albumin binders (S)-2b and (S)-2c............ 6

Discussion S3: $\quad$ Association and dissociation experiments .......................................... 7

Figure S1: $\quad$ Binding curves obtained with MST and fluorescence-based competition assay for the different albumin binders ........................... 9

Figure S2: $\quad$ Results for the SDS denaturation tests.............................................. 14

Figure S3: $\quad$ Relationship between the electronic properties $\left(\sigma_{p}\right.$ and $\left.\sigma_{p-}\right)$ of the substituents in para position of the phenyl residue and the $K_{d}$ values of the albumin binders $(S)-1 f-(S)-11$

Figure S4: $\quad$ HSA binding curve for $\left[{ }^{64} \mathrm{Cu}\right] \mathrm{Cu}-(S)-10$ obtained by the

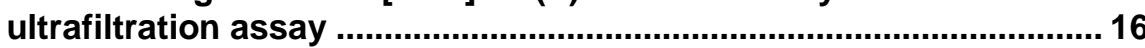

Figure S5: $\quad$ Saturation binding curves for the different ${ }^{64} \mathrm{Cu}$-labeled TATE derivatives towards $\mathrm{SST}_{2}$ using MPC cell lysates.............................. 17

Figure S6: $\quad$ Non-specific binding curves ............................................................... 19

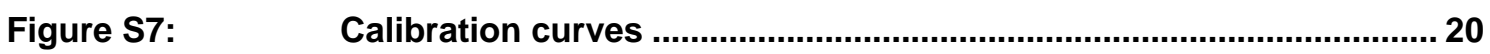

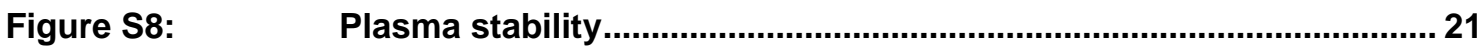

Figure 59: $\quad$ Time-dependent SUV data of muscle and liver for the different ${ }^{64} \mathrm{Cu}$-labeled TATE derivatives and the isolated albumin binder....... 23

Figure S10: $\quad$ Association experiments for $\left[{ }^{64} \mathrm{Cu}\right] \mathrm{Cu}$-NODAGA-TATE (Left column) and $\left[{ }^{64} \mathrm{Cu}\right] \mathrm{Cu}-5$ (right column) ......................................................... 25

Figure S11: Dissociation experiments für $\left[{ }^{64} \mathrm{Cu}\right] \mathrm{Cu}-\mathrm{NODAGA}-\mathrm{TATE},\left[{ }^{64} \mathrm{Cu}\right] \mathrm{Cu}-5$

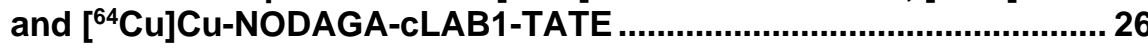

Table S1: $\quad$ SUV data for the different ${ }^{64} \mathrm{Cu}$-labeled TATE derivatives and the isolated albumin binder .................................................................... 27

Table S2: $\quad$ Summary of calculated $A A_{0-48 h}$ values from plots of SUV=f(time) for the different ${ }^{64} \mathrm{Cu}$-labeled TATE derivatives and the isolated albumin binder

Table S3: $\quad$ Summary of kinetic parameters for selected ${ }^{64} \mathrm{Cu}$-labeled

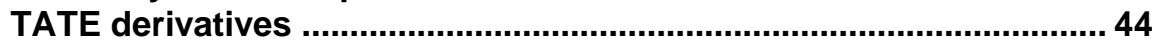

Scheme S1: $\quad$ Synthesis of NHS ester 7a-c ........................................................... 45

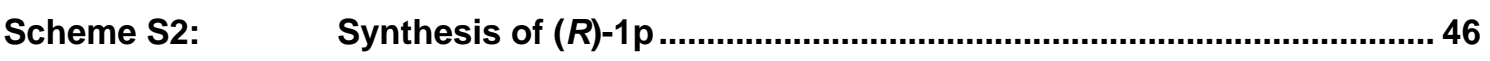

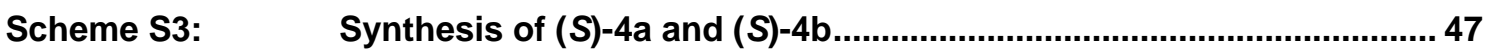

Scheme S4: $\quad$ Synthesis of NODAGA-cLAB-TATEs ......................................................... 48

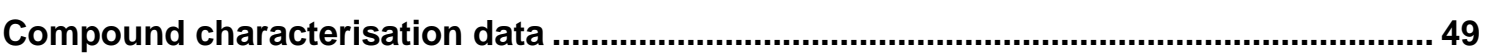

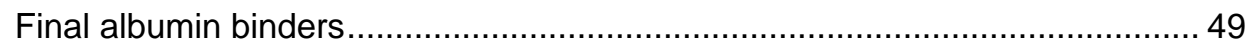

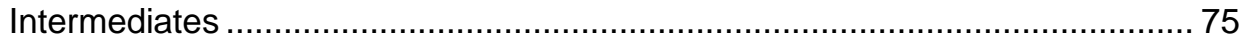

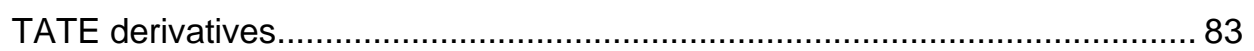

${ }^{64} \mathrm{Cu}$-labeled TATE derivatives and the isolated albumin binder $(S)-10 \ldots \ldots \ldots . . .95$

References 


\section{Discussion S1: Synthesis of the albumin binders}

\section{Solid-phase synthesis}

The basis for the clickable albumin binders (CLABs) in the present study represent a lysine derivative with unsymmetric substitution pattern at the $\alpha$ - and $\varepsilon$-amino groups and a free carboxyl group (Acetyl-Lys(IPB)-OH). For a rapid access to such compounds, a solid-phase synthetic strategy was developed starting from commercially available Fmoc-Lys(Alloc)-OH or Alloc-Lys(Fmoc)-OH which were anchored onto the 2-chlorotrityl chloride (2-CITrtCl) resin (Scheme 1 within main article). Concerning the use of Fmoc-Lys(Alloc)-OH, the a-amino group should be functionalized prior to the $\varepsilon$-amino group as the latter one is able to mediate the Fmoc deprotection at the former one. ${ }^{1}$ With the exception of the loading step and acetylations using acetic anhydride, all synthesis steps including Alloc deprotection were performed in an automated microwave peptide synthesizer (Biotage Initiator+ Alstra). In this context, the feasibility of the microwave assisted Alloc deprotection without inert gas atmosphere was recently demonstrated by Wilson et al., who applied two cycles of short microwave irradiation (each $5 \mathrm{~min}$ ) with 0.25 eq. of $\mathrm{Pd}\left(\mathrm{PPh}_{3}\right)_{4}$ and 15 eq. of phenylsilane tempered at $38^{\circ} \mathrm{C}$ in a CEM Liberty Blue automated microwave synthesizer. ${ }^{2}$ Moreover, a blog post by Biotage confirmed the applicability of the microwave assisted Alloc deprotection using the Initiator+ Alstra device. ${ }^{3}$ Inspired by these reports, we programmed the Alloc deprotection cycle for our device employing the same equivalents for catalyst and scavenger and similar conditions $(2 \times 10 \mathrm{~min}$, $40^{\circ} \mathrm{C}$ ) as described by Wilson et al. However, we used a single solution of both reagents in $\mathrm{CHCl}_{3}$ (freshly prepared maximal 30 min prior to the reaction). In this context, $\mathrm{CHCl}_{3}$ proved to be more suitable than $\mathrm{CH}_{2} \mathrm{Cl}_{2}$ due to the higher boiling point. $\mathrm{A}$ repeated passage of deprotection and acylation and subsequent HFIP-mediated cleavage from the resin provided the desired lysine-derived albumin binders in moderate yields (20-40\%, Scheme 1 within main article) and amounts $(20-60 \mathrm{mg})$. In addition to the FAM-conjugate $(\boldsymbol{R})-\mathbf{1 a}$ and the $\boldsymbol{N}^{\mathbf{a}}$ acetylated lysine-derivatives $(\boldsymbol{R})$-1c and (S)-1d, which were already described by Dumelin et al. ${ }^{4}$ and served as fluorescent probe and reference compounds herein, the TAMRA conjugate $(R)-1 b$ and the CLABs $(R)-1 d$ and (S)-1e bearing 4-pentynoyl and 5-azidopentanoyl residues, respectively, were synthesized. Furthermore, the C-terminally amidated analogue of (S)-1C, (S)-2a, was prepared using the Rink Amide resin instead of the 2-CITrtCl resin to characterize the effect of a carboxamide group on the binding affinity to albumin. 
Synthesis in solution for carboxylic acids and hybrid solid-phase/solution synthesis for carboxamides

After prove of unaltered albumin binding affinity of $(\boldsymbol{R})$-1d and $(\boldsymbol{S})$-1e compared to the reference compounds $(R)-1 \mathrm{C}$ and $(S)-1 \mathrm{C}$, we decided to switch the synthetic strategy from solid-phase to synthesis in solution to improve the yields and to prepare the albumin binders in larger scale for future functionalization of target molecules. To reduce the synthesis steps to a minimum, we initially started from free lysine (Scheme 2 within main article). Complexation of the $\alpha$-amino group and the carboxyl group by $\mathrm{Cu}^{2+}$ under basic conditions and subsequent application of 4(4-iodophenyl)butanoic acid NHS ester (IPB-NHS, separately prepared, see Scheme S1) enabled the selective functionalization of the $\varepsilon$-amino group. ${ }^{5}$ The resulting copper complex has been isolated by filtration. The release of copper was achieved by dissolution and heating of the complex in an aqueous solution of EDTA to furnish D-Lys(IPB)-OH ((R)-6). Subsequent acylation at the $\alpha$-amino group was possible by reaction with the NHS esters of carboxylic acids (Scheme S1). Compounds $(\boldsymbol{R})$-1e and $(\boldsymbol{R})$-1f bearing 5-azidopentanoyl and 4azidobenzoyl residue at the $\alpha$-amino group, respectively, were prepared via this synthetic route. Even though this route requires only two reaction steps and no intermediate purifications for the unsymmetric functionalization (in addition, the NHS ester had to be prepared), the low yields of Lys(IPB)-OH, the high amounts of EDTA necessary for the release of copper and the very time-consuming filtrations led us to reconsider this synthetic strategy. Worth of note, while the compounds with aliphatic acyl residues at the $\alpha$-amino group were oils, compound $(\boldsymbol{R})$-1f with a 4-azidobenzoyl residue crystallized well and has been obtained as white solid. As this residue turned out to be also advantageously for the binding affinity to albumin, it was mainly considered for the functionalization at the a-amino group within the following synthetic approach. Furthermore, we decided to focus on L-lysine instead of D-lysine as the (S)configuration is slightly preferred regarding the binding affinity to HSA.

For our most favorable synthetic approach, we chose either Boc-L-Lys-OH (for the series of $N^{*}-$ IPB derivatives) or L-Lys(Boc)-OH (for the series of $N^{a}-4-N_{3} B z$ derivatives) as starting materials, which allowed for the preparation of the lysine-based albumin binders with free carboxyl group in three steps (Scheme 3 within main article). For the $N^{k}$-acylation with aliphatic carboxylic acids, the respective NHS esters were used which were generated in situ with DIC and NHS (including a subsequent filtration step to remove precipitated diisopropylurea). The acylation was performed in an aqueous milieu with $\mathrm{Na}_{2} \mathrm{CO}_{3}$ as base (Schotten-Baumann conditions). For the $N^{a}$-acylation, a similar procedure to the $N^{k}$-acylation was chosen. However, for benzoic acids the in situ activation as HOBt ester using DCC and HOBt led to higher yields for the acylation than the use of the respective NHS ester (40-50\% compared to 10-15\%). The 
intermediates Boc-L-Lys(IPB)-OH ((S)-8) and 4-N ${ }_{3}$ Bz-L-Lys(Boc)-OH ((S)-9) were purified by column chromatography and were obtained in good yields (78 and $79 \%$, respectively). After TFA-mediated removal of the Boc-group, the amphoteric mono-acylated lysines (L-Lys(IPB)$\mathrm{OH}((S)-6)$ and 4-N $\mathrm{N}_{3} \mathrm{Bz}-\mathrm{L}-\mathrm{Lys}-\mathrm{OH}((\boldsymbol{S})-10)$ were isolated by lyophilization after addition of water. Finally, acylation of the free amino group was performed as mentioned above. By using this synthetic strategy ten lysine-based albumin binders with free carboxyl group were synthesized

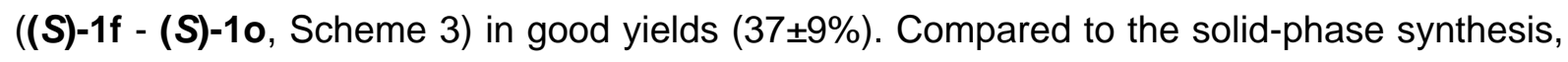
this procedure can easily adapted to higher scales.

For the synthesis of lysine carboxamides, a hybrid solid-phase/solution synthesis was elaborated (Scheme 3 in main article) that is more convenient than performing the entire synthesis at the polymeric support using Fmoc-Lys(Alloc)-OH (Scheme 1). The initial loading of Fmoc-L-Lys(Boc)-OH on the Rink-amide resin represents a simple way to lysine carboxamides. Furthermore, Fmoc-deprotection can be easily realized on resin using $20 \%$ piperidine/DMF and after $N^{\alpha}$-acylation Boc removal and release from resin can be performed simultaneously using TFA. Final $N^{\varepsilon}$-acylation was achieved without prior purification. Compounds (S)-2b and (S)-2c were synthesized by this strategy. In addition to the effect of Cterminally amidation on the HSA binding affinity, the effect of converting the carboxylic acid to its methyl ester was of interest. For this purpose, compound (S)-3a was synthesized in solution starting from Boc-L-Lys-OMe in three steps analogously to the synthesis of the carboxylic acids 1. For the lysine carboxamides and the methyl esters, the preactivation of the carboxylic acids as active esters were replaced by the coupling agents HATU or PyBOP.

In addition to the incorporation of azide-bearing acyl residues at the a-amino group, we hypothesized that $\mathrm{N}_{3}$-Lys(IPB)-OH would also be a useful building block for the introduction of an albumin binding moiety. To prove this, we synthesized the respective click product with phenylacetylene, $(\boldsymbol{R})-\mathbf{1 p}$, starting from $\mathrm{N}_{3}$-D-Lys(Boc)-OH in three steps (CuAAC, Boc removal, $N^{\varepsilon}$-acylation, see Scheme S2). 


\section{Discussion S2: Prediction of $K_{d}$ values for albumin binders (S)-2b and (S)-2c}

Based on the factor ( $f=22)$ between the $K_{d}$ values of $(S)-1 c$ (Acetyl-L-Lys(IPB)-OH, 3.2 $\left.\mu \mathrm{M}\right)$ and (S)-2a (Acetyl-L-Lys(IPB)-NH $\left.\mathbf{N H}_{2}, 70 \mu \mathrm{M}\right)$, the $K_{d}$ value of (S)-2b ( $\mathbf{N}_{3}$-Bz-L-Lys(IPB)- $\left.\mathbf{N H}_{2}\right)$ can be estimated to $7.9 \mu \mathrm{M}\left(=22 \times 0.36 \mu \mathrm{M}, K_{d}\right.$ value of its carboxylic acid analog $)$ by assuming additive structure-activity relationships. Similarly, the $K_{d}$ value of (S)-2c ( $\mathbf{N}_{3}$-Bz-L-Lys(MPB)$\left.\mathrm{NH}_{2}\right)$ can be estimated to $66 \mu \mathrm{M}\left(=22 \times 3.0 \mu \mathrm{M}, K_{d}\right.$ value of its carboxylic acid analog $)$. 


\section{Discussion S3: Association and dissociation experiments}

To get an impression on the binding kinetics of the TATE derivatives in terms of microscopic rate constants, we performed association and dissociation experiments for selected peptides ([ $\left[{ }^{64} \mathrm{Cu}\right] \mathrm{Cu}-N O D A G A-T A T E, \quad\left[{ }^{64} \mathrm{Cu}\right] \mathrm{Cu}-5$ and $\left.\left[{ }^{64} \mathrm{Cu}\right] \mathrm{Cu}-N O D A G A-c L A B 1-T A T E\right)$. The association process was followed over $3 \mathrm{~h}$ at three different concentrations $(0.625 \mathrm{nM}, 2.5 \mathrm{nM}$ and $20 \mathrm{nM}$ ) for the first two peptides (Figure S10). The rate constants $k_{\text {obs }}$ were obtained from the time curves (total binding) by nonlinear regression using equation SI (one-phase association in Prism), ${ }^{6}$

$$
\mathrm{CPM}=\mathrm{CPM}_{0}+\left(\mathrm{CPM}_{\mathrm{eq}}-\mathrm{CPM}_{0}\right) *\left(1-\mathrm{e}^{-k_{\mathrm{obs}} * \mathrm{t}}\right)
$$

where CPM (counts per minute) denotes the measured radioactivity at start of radioligand addition $\left(\mathrm{CPM}_{0}\right)$ and when the equilibrium $\left(\mathrm{CPM}_{\mathrm{eq}}\right)$ is reached. Replotting the $k_{\mathrm{obs}}$ values versus the radioligand concentrations yields hyperbolic curves (Figure S10), which indicates a twostep binding process (common kinetic model for high-affinity receptor-ligands interactions, Table S3). ${ }^{7}$ Analysis of these curves was performed by nonlinear regression using equation SII. ${ }^{7}$

$$
k_{\mathrm{obs}}=k_{\mathrm{off}, 2}+\frac{k_{\mathrm{on}, 2} *[\mathrm{~L}]}{K_{\mathrm{d}}+[\mathrm{L}]}
$$

This allows for the derivation of the microscopic rate constants for the second binding step of the radioligand-receptor interaction (Table S3). The obtained values for $k_{\mathrm{on}, 2}$ are similar for both radioligands $\left(0.051 \mathrm{~min}^{-1}\right.$ and $0.060 \mathrm{~min}^{-1}$ for $\left[{ }^{64} \mathrm{Cu}\right] \mathrm{Cu}-N O D A G A-T A T E$ and $\left[{ }^{64} \mathrm{Cu}\right] \mathrm{Cu}-5$, respectively). However, for $k_{\text {off, }, 2}$, the values differ significantly $\left(0.017 \mathrm{~min}^{-1}\right.$ and $0.002 \mathrm{~min}^{-1}$ for $\left[{ }^{64} \mathrm{Cu}\right] \mathrm{Cu}-N O D A G A-T A T E$ and $\left[{ }^{64} \mathrm{Cu}\right] \mathrm{Cu}-5$, respectively) indicating that the half-life of the $\left[{ }^{64} \mathrm{Cu}\right] \mathrm{Cu}-5$-receptor complex is greater compared to the $\left[{ }^{64} \mathrm{Cu}\right] \mathrm{Cu}-$ NODAGA-TATE-receptor complex.

In addition to association experiments, we performed also dissociation experiments (Figure S11). To this end, MPC cell lysate was incubated with $20 \mathrm{nM}$ radioligand for $1 \mathrm{~h}$ and the dissociation was induced by addition of $20 \mu \mathrm{M}$ non-radioactive peptide (1000-fold excess). ${ }^{6}$ Nonlinear regression of these curves using equations for one-phase or two-phase exponential decay (as implemented in GraphPad Prism) allows for the determination of the rate constants for dissociation. [ ${ }^{64} \mathrm{Cu}$ ]Cu-NODAGA-TATE shows a dissociation behavior best described by the one-phase model with a rate constant of $0.012 \mathrm{~min}^{-1}$, which is in accordance to the $k_{\text {off,2 }}$ value from the association experiment $\left(0.017 \mathrm{~min}^{-1}\right)$. Therefore, one-phase dissociation indicates that $k_{\text {off, } 1}>>k_{\text {off, } 2}$ for $\left[{ }^{64} \mathrm{Cu}\right] \mathrm{Cu}$-NODAGA-TATE. In contrast, $\left[{ }^{64} \mathrm{Cu}\right] \mathrm{Cu}-5$ and also $\left[{ }^{64} \mathrm{Cu}\right] \mathrm{Cu}-$ NODAGA-cLAB1-TATE show a dissociation behavior which is best described by 
the two-phase model. The greater rate constants might reflect $k_{\text {off, } 1}\left(0.150\right.$ and $\left.0.300 \mathrm{~min}^{-1}\right)$ and the lower $k_{\text {off, } 2}\left(0.005\right.$ and $0.015 \mathrm{~min}^{-1}$ for $\left[{ }^{64} \mathrm{Cu}\right] \mathrm{Cu}-5$ and $\left[{ }^{64} \mathrm{Cu}\right] \mathrm{Cu}-N O D A G A-c L A B 1-T A T E$, respectively). Again, the $k_{\text {off,2 }}$ value for $\left[{ }^{64} \mathrm{Cu}\right] \mathrm{Cu}-5$ is in accordance to the value determined from the association experiment $\left(0.005 \mathrm{~min}^{-1}\right.$ versus $\left.0.002 \mathrm{~min}^{-1}\right)$. Therefore, $k_{\text {off }, 1}$ is greater than $k_{\text {off, } 2}$ for these two radioligands (ratio of 30 and 20), but the ratio seems to be lower than for $\left[{ }^{64} \mathrm{Cu}\right] \mathrm{Cu}-\mathrm{NODAGA-TATE}$, which accounts for the detected two-phase dissociation. Consequently, the $k_{\mathrm{off}, 1}$ value of $\left[{ }^{64} \mathrm{Cu}\right.$ ]Cu-NODAGA-TATE (and also the $k_{\mathrm{on}, 1}$ value) might be significantly greater compared to the other two peptides. This seems to be reasonable as $\left[{ }^{64} \mathrm{Cu}\right] \mathrm{Cu}-5$ and $\left[{ }^{64} \mathrm{Cu}\right] \mathrm{Cu}-\mathrm{NODAGA}-\mathrm{cLAB1}-\mathrm{TATE}$ exhibit a greater steric demand, which could affect the formation of the initial encounter complex $(R L)$ by slowing down the association and the dissociation process. In accordance to that, a two-phase dissociation was also observed by Zeggari et al. for a ${ }^{125}$ l-labeled somatostatin 28 analog ( ${ }^{125}$ I-[Leu ${ }^{8}$, D-Trp ${ }^{22}$, $\mathrm{Tyr}^{25} \mathrm{~S}_{28}$; binds non-selectively to SST subtypes $\left.1-5\right)^{8}$ in binding studies using guinea pig pancreatic acinar cell plasma membranes. ${ }^{9}$ 
Figure S1: Binding curves obtained with MST and fluorescencebased competition assay for the different albumin binders

For $K_{d}$ values and further information see Table 1 and experimental section within the main article.
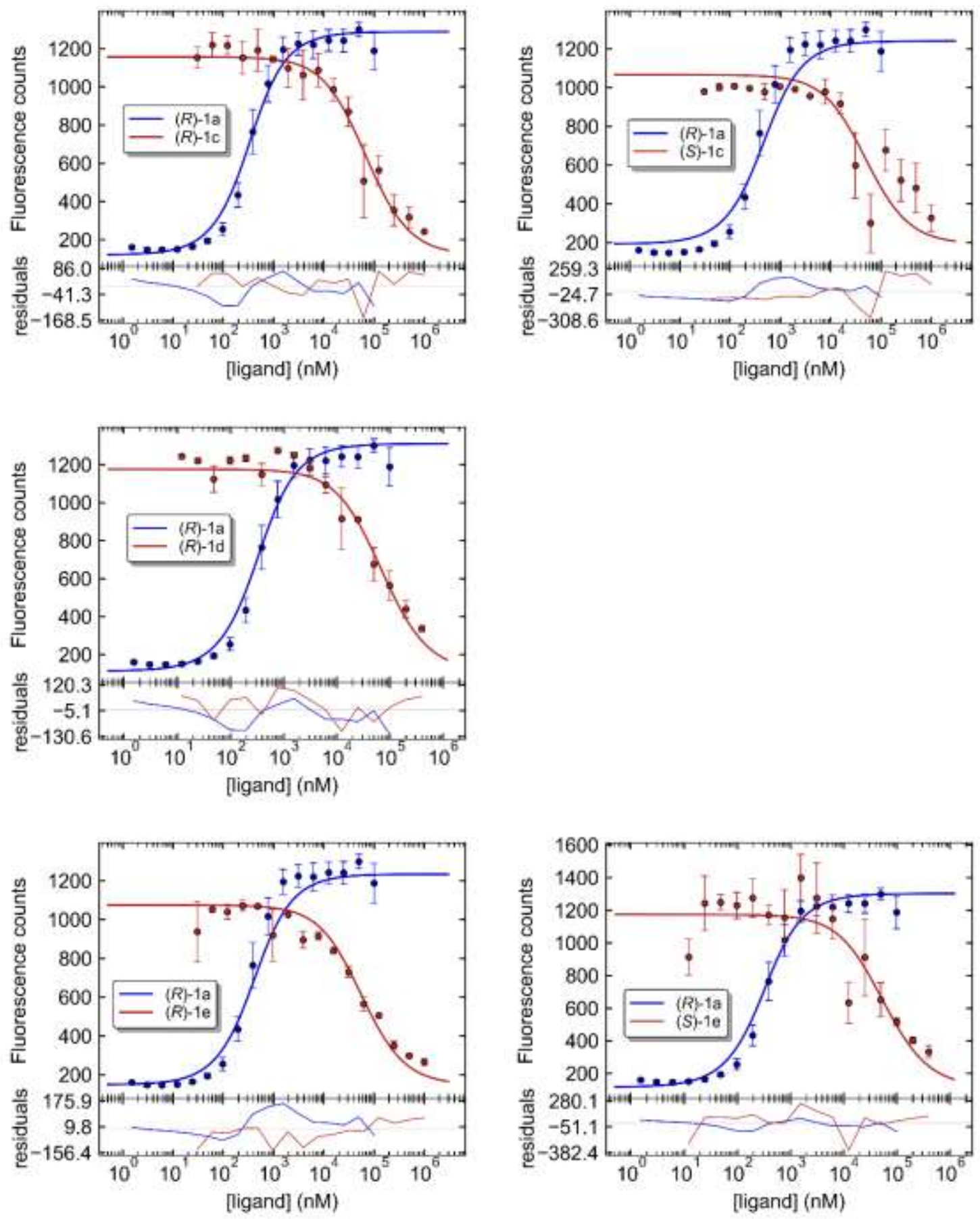

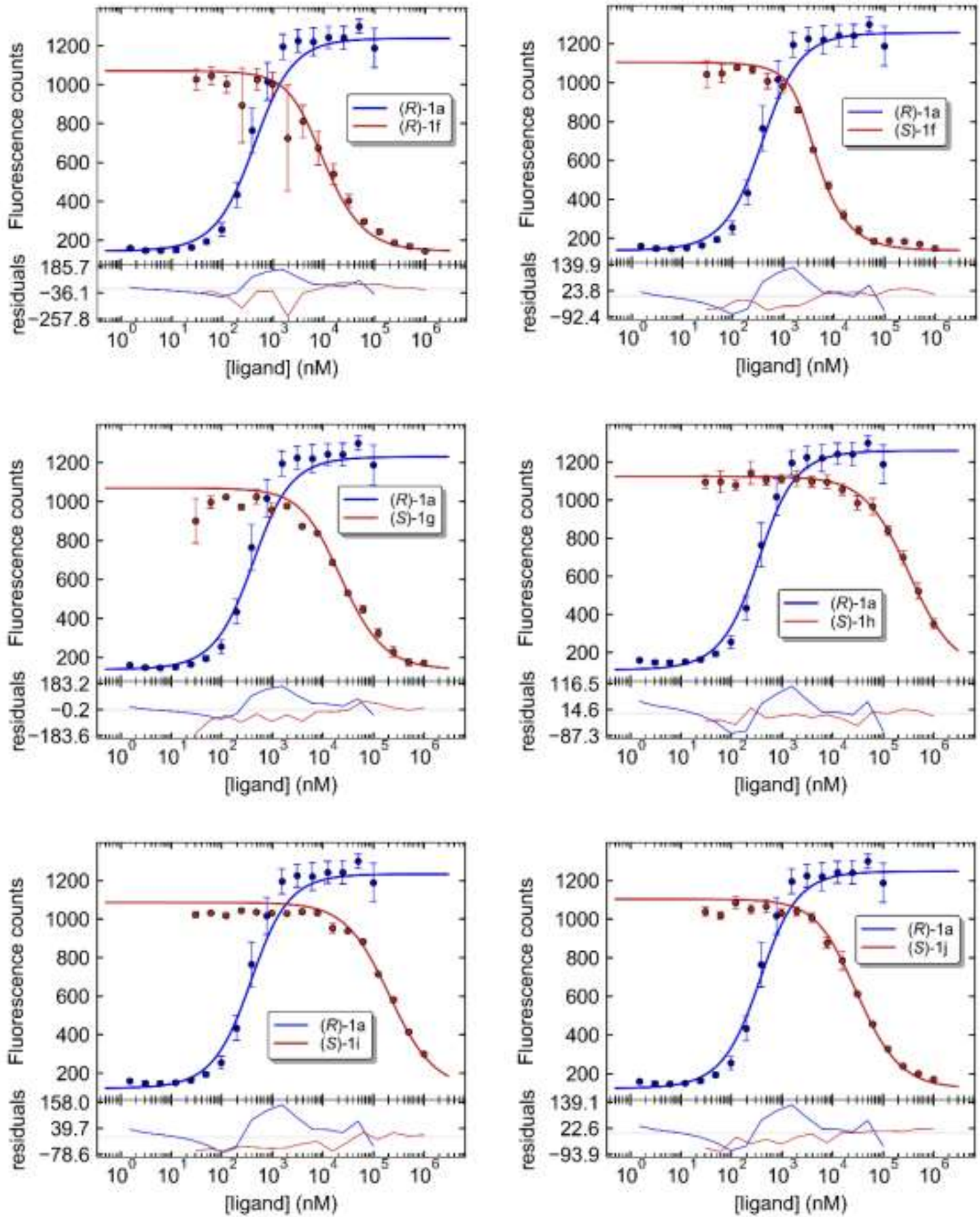

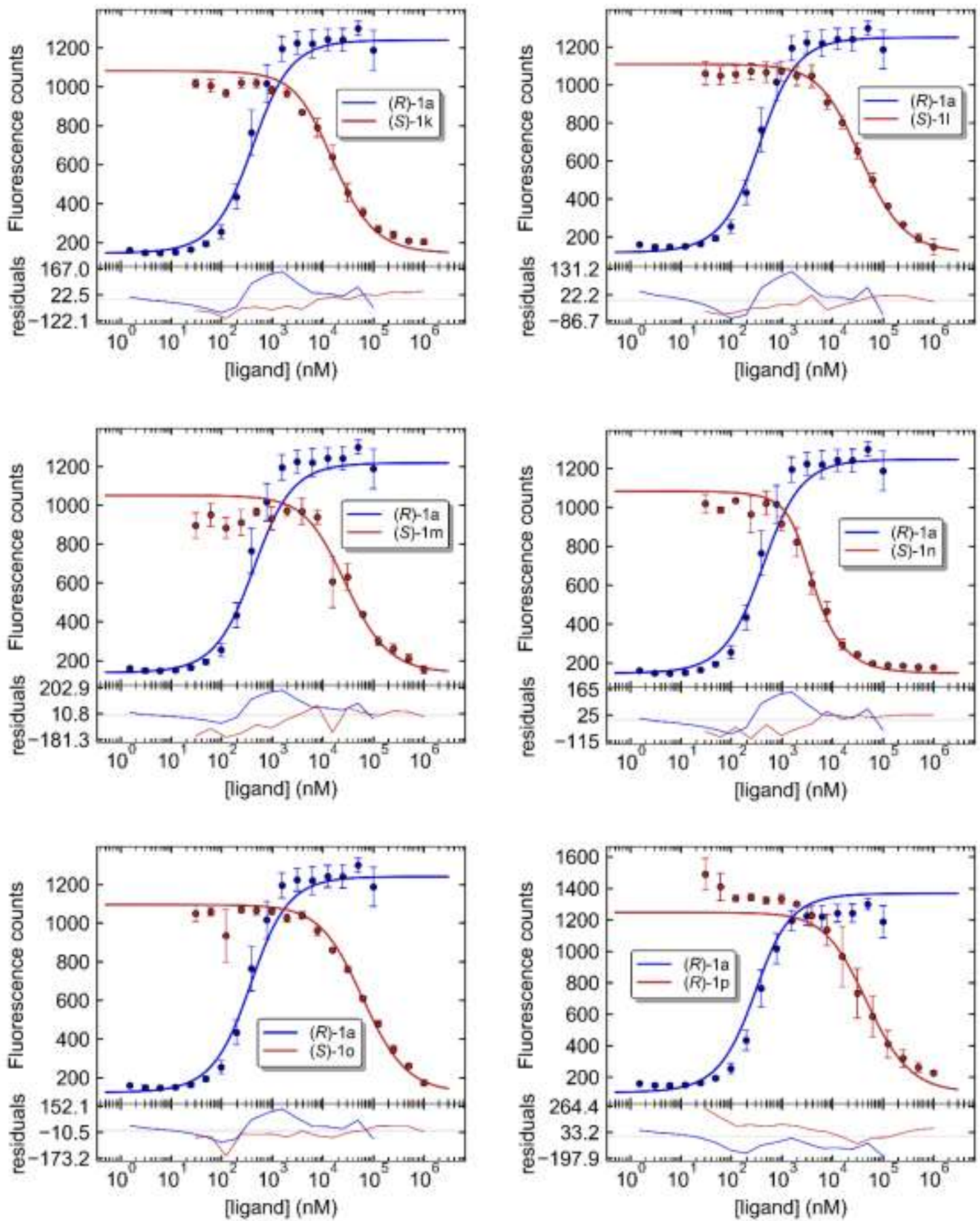

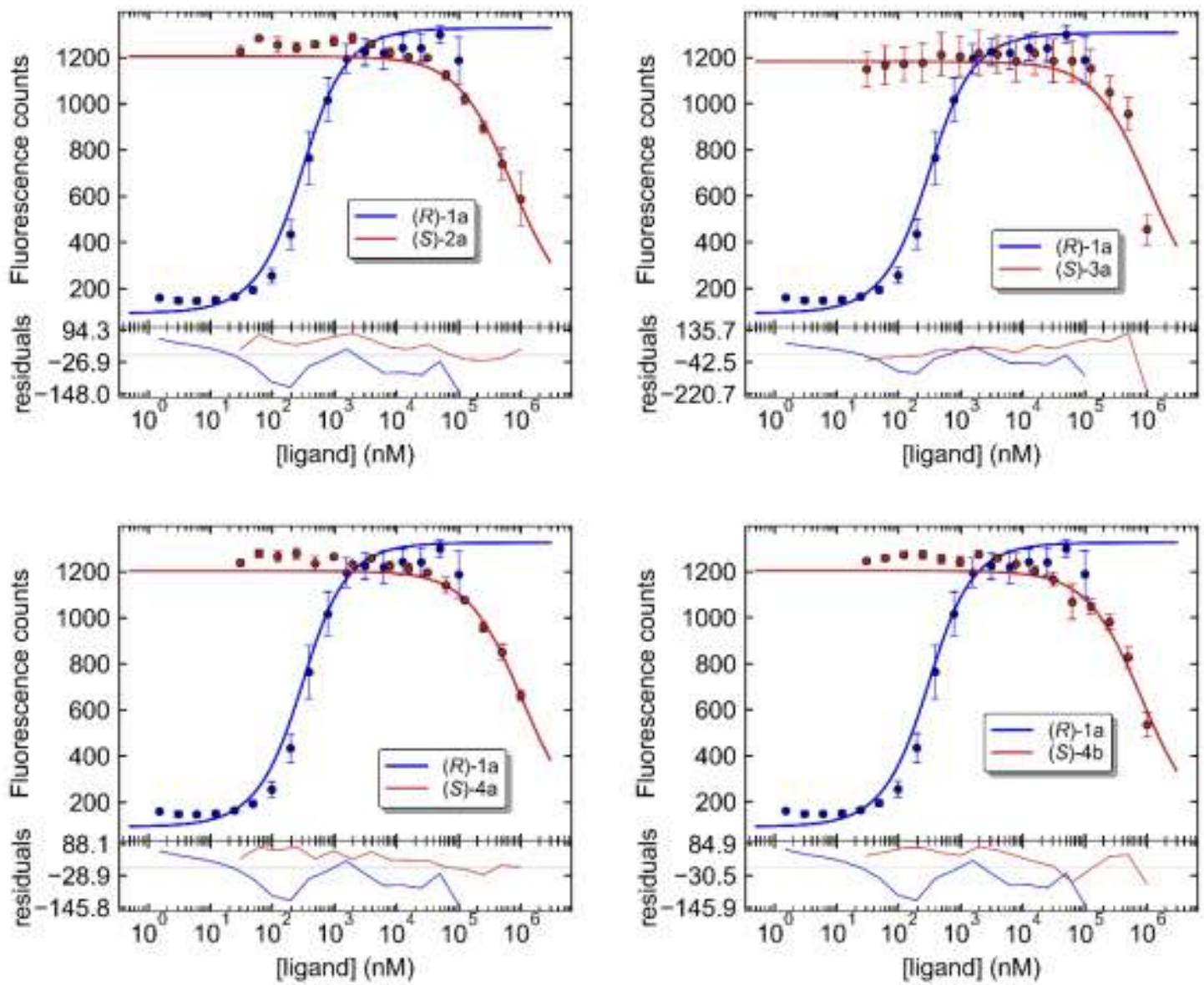

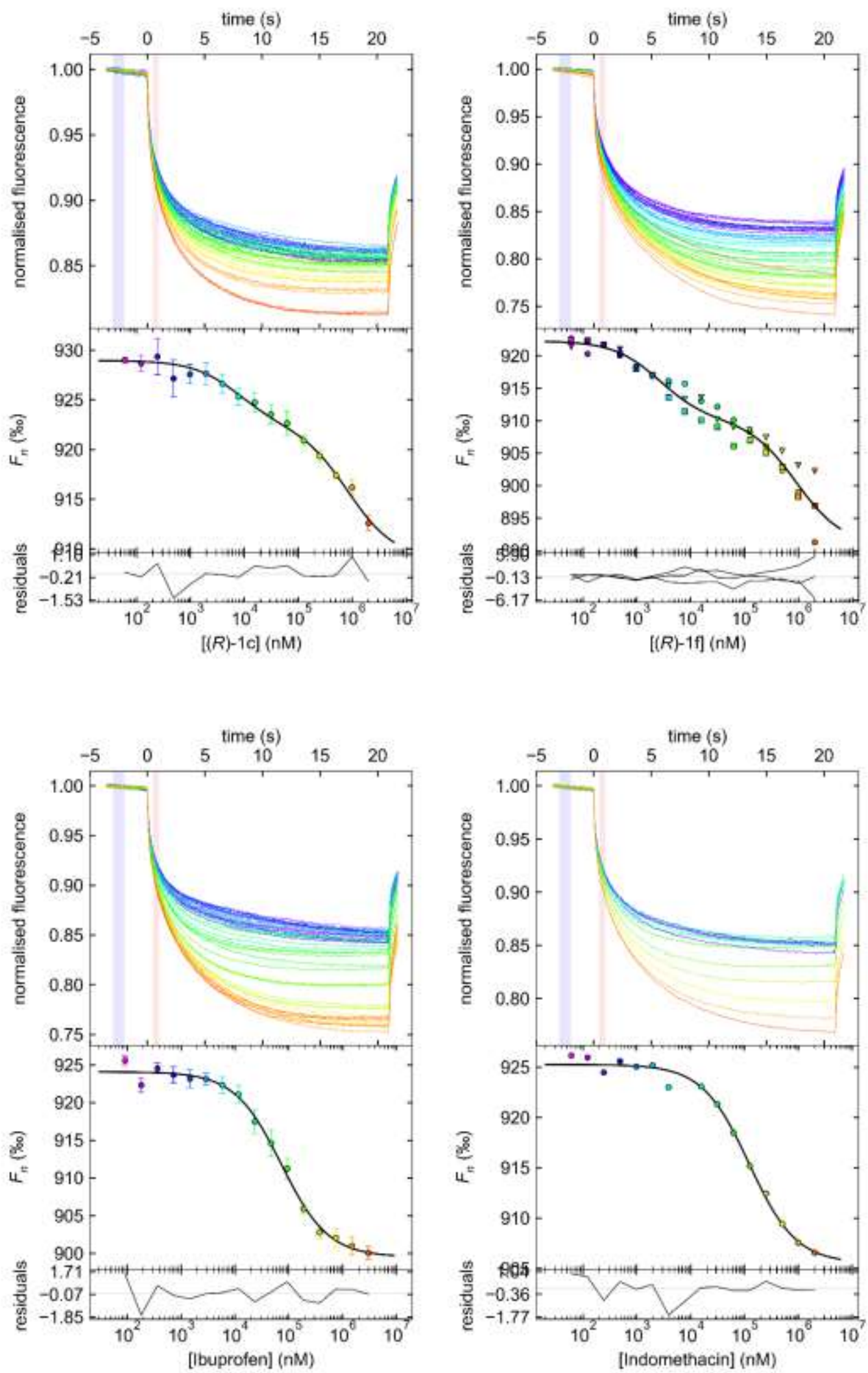


\section{Figure S2: Results for the SDS denaturation tests}

The SDS denaturation test was performed for both fluorophore-labeled albumin binders to determine the specificity of the fluorescence increase in the presence of HSA according to the manufacturer's instruction (denaturation by addition of a solution containing 4\% SDS and 40 $\mathrm{mM} \mathrm{DTT}$ and incubation for $5 \mathrm{~min}$ at $\left.95^{\circ} \mathrm{C}\right)$. As seen below, the fluorescence increase for $(\boldsymbol{R})$ 1a does not occur after denaturation of HSA while for $(\boldsymbol{R}) \mathbf{- 1 b}$ the increase persists which indicates a binding-specific fluorescence increase for $(\boldsymbol{R})$-1a but not for $(\boldsymbol{R}) \mathbf{- 1} \mathbf{b}$. 'Target' refers to a solution of the albumin binder without HSA and 'complex' refers to a solution of albumin

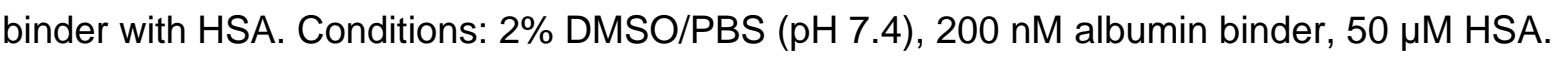

\section{Result for 6-FAM conjugate $(R)-1 a$}
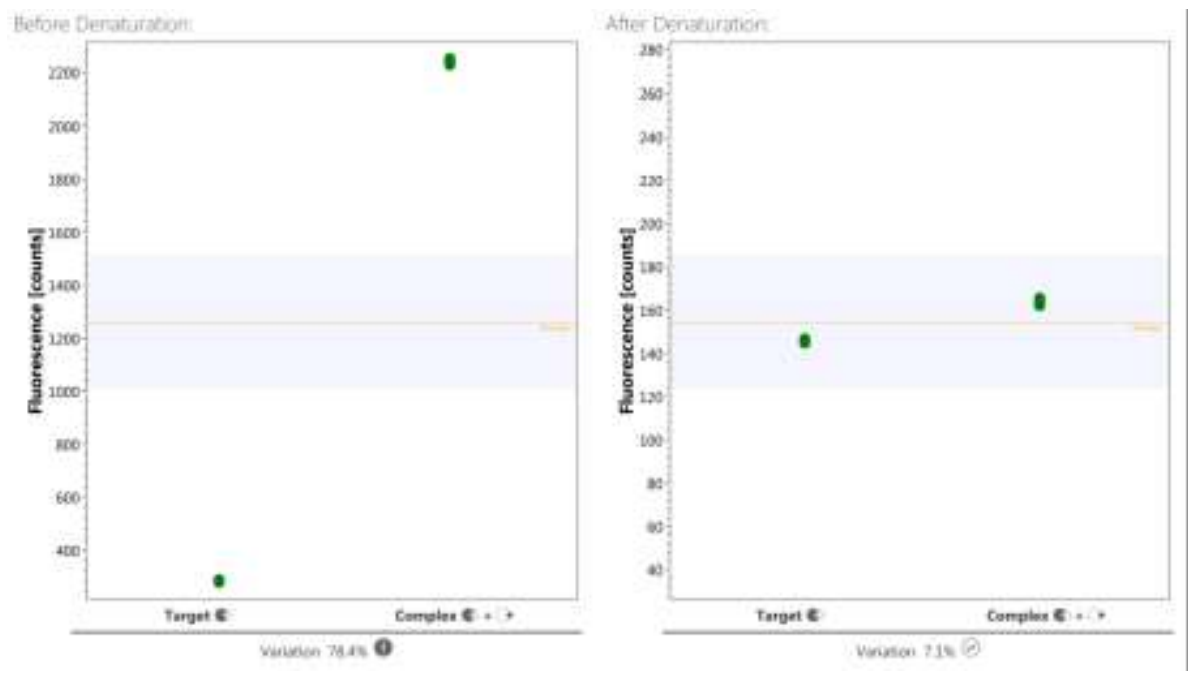

\section{Result for 6-TAMRA conjugate $(R)-1 \mathrm{~b}$}
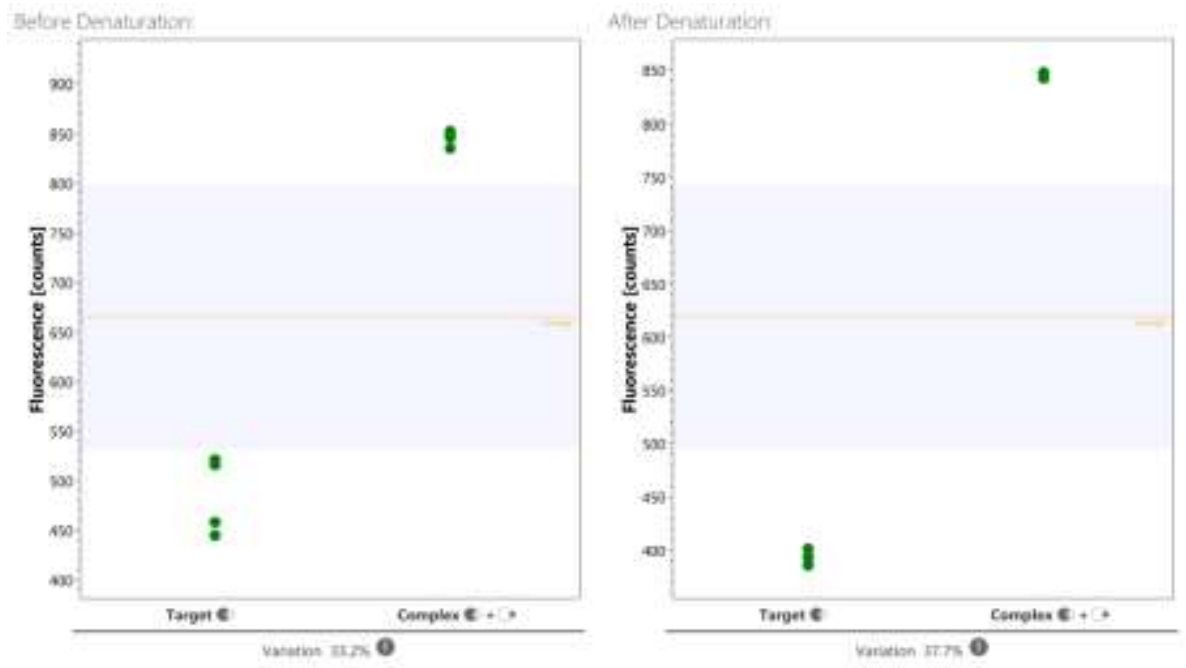
Figure S3: Relationship between the electronic properties ( $\sigma_{\mathrm{p}}$ and $\left.\sigma_{p-}\right)$ of the substituents in para position of the phenyl residue and the $K_{d}$ values of the albumin binders $(S)-1 f-(S)-11$

Plots of $\log \left(K_{\mathrm{d}}\right)=\mathrm{f}\left(\sigma_{\mathrm{p}}\right)($ left $)$ and $\log \left(K_{\mathrm{d}}\right)=\mathrm{f}\left(\sigma_{\mathrm{p}-}\right)$ (right) using the mean values of the $K_{\mathrm{d}}$ values $(\mathrm{nM})$ of compounds (S)-1f $-(\mathbf{S})-\mathbf{1 l}$ (Table 1 in main article) and the following values for $\sigma_{\mathrm{p}}:{ }^{10} 0.00$ $(\mathrm{H}), 0.06(\mathrm{~F}),-0.17\left(\mathrm{CH}_{3}\right), 0.23(\mathrm{Cl}), 0.23(\mathrm{Br}), 0.18(\mathrm{I}), 0.54\left(\mathrm{CF}_{3}\right)$; and $\sigma_{\mathrm{p}-\mathrm{-}^{10}, 11} 0.00(\mathrm{H}),-0.03$ $(\mathrm{F}),-0.17\left(\mathrm{CH}_{3}\right), 0.19(\mathrm{Cl}), 0.25(\mathrm{Br}), 0.65\left(\mathrm{CF}_{3}\right), 0.27(\mathrm{I})$. Regression analysis was performed by linear regression; $n$ denotes to the amount of data points, $s$ to the standard deviation of the regression equation, and $\mathrm{R}^{2}$ to the coefficient of determination. ${ }^{12,13}$
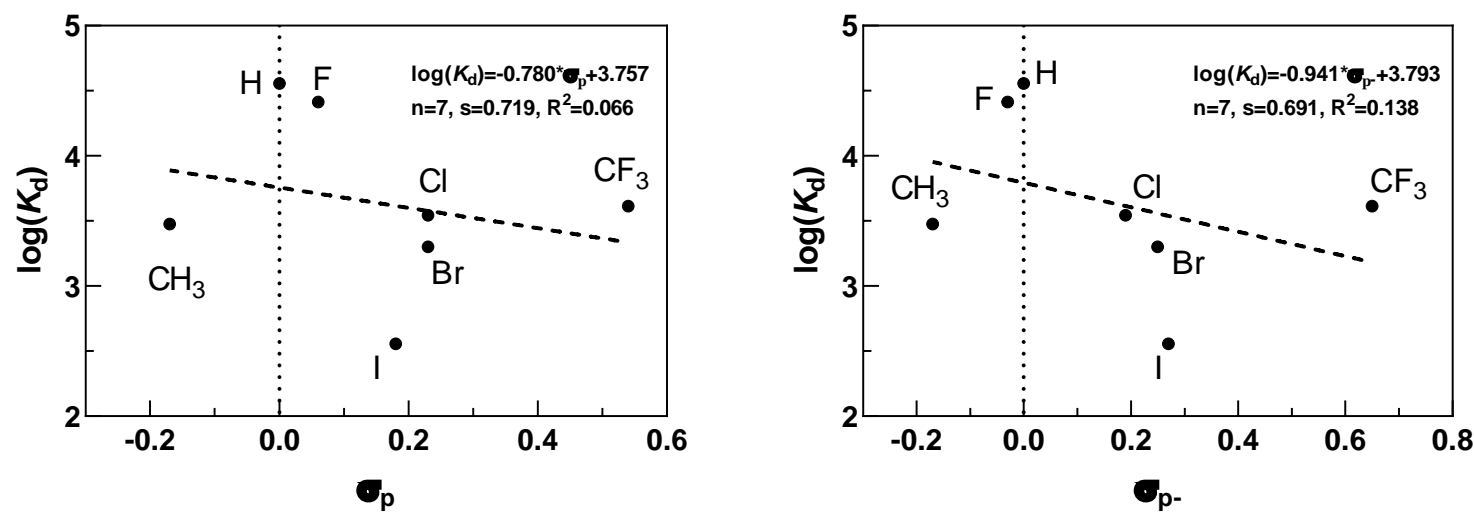
Figure S4: HSA binding curve for $\left[{ }^{64} \mathrm{Cu}\right] \mathrm{Cu}-(S)-10$ obtained by the ultrafiltration assay

Plot of "fraction of retained activity" $=\mathrm{f}([\mathrm{HSA}])$ with nonlinear regression using equation I (Morrison equation, see main article). Data shown are from one single experiment.

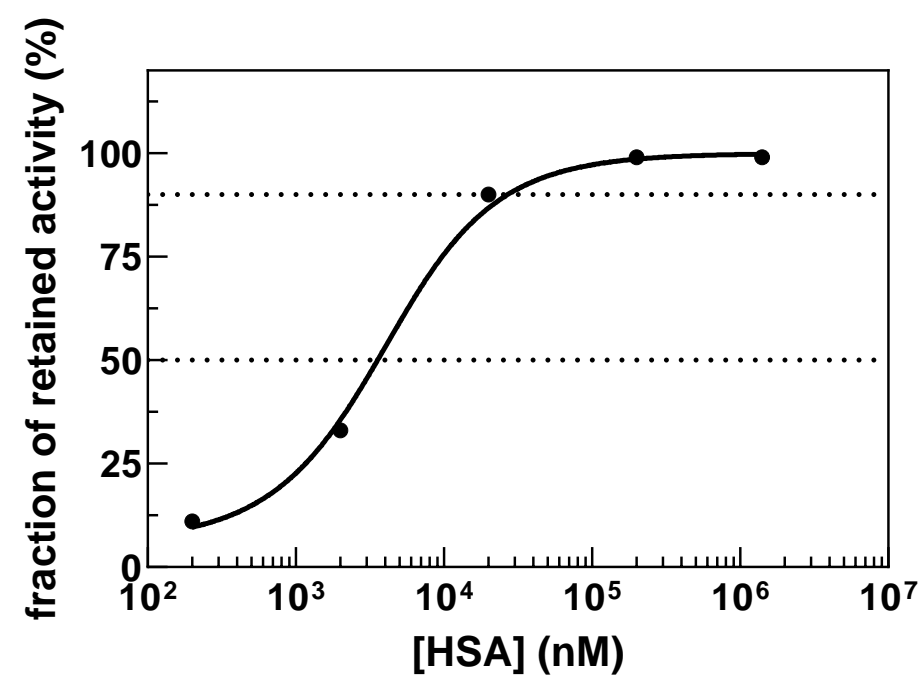




\section{Figure S5: Saturation binding curves for the different ${ }^{64} \mathrm{Cu}$-labeled TATE derivatives towards $\mathrm{SST}_{2}$ using MPC cell lysates}

Plots of 'binding' $=f$ (radioligand) with nonlinear regressions using the model of 'One site - Total, accounting for ligand depletion' as implemented in GraphPad Prism for the data of total binding and linear regressions for the data of non-specific binding. For the ligand depletion model, the term 'NS' was constrained to the respective slopes obtained by the linear regressions. The terms 'SpecAct' (for determination see Figure S7) and 'Vol' ( $0.2 \mathrm{~mL}$, assay volume) were also constrained. $K_{\mathrm{d}}$ values were derived in $\mathrm{nM}$ and the $B_{\max }$ values (in $\mathrm{cpm}$ ) were transformed into $\mathrm{fmol} / \mathrm{mg}$ (Table 3 within main article). Both data sets were corrected for filter binding. Data shown for total binding are mean values $( \pm S D)$ of one experiment, which was performed in duplicate (single assay for non-specific data). Incubation period of $1 \mathrm{~h}$. Non-specific binding was determined in the presence of $1 \mu \mathrm{M}$ DOTA-TATE. Radioligand binding was between 17$58 \%$ at the lowest radioligand concentration $(0.625 \mathrm{nM})$. 

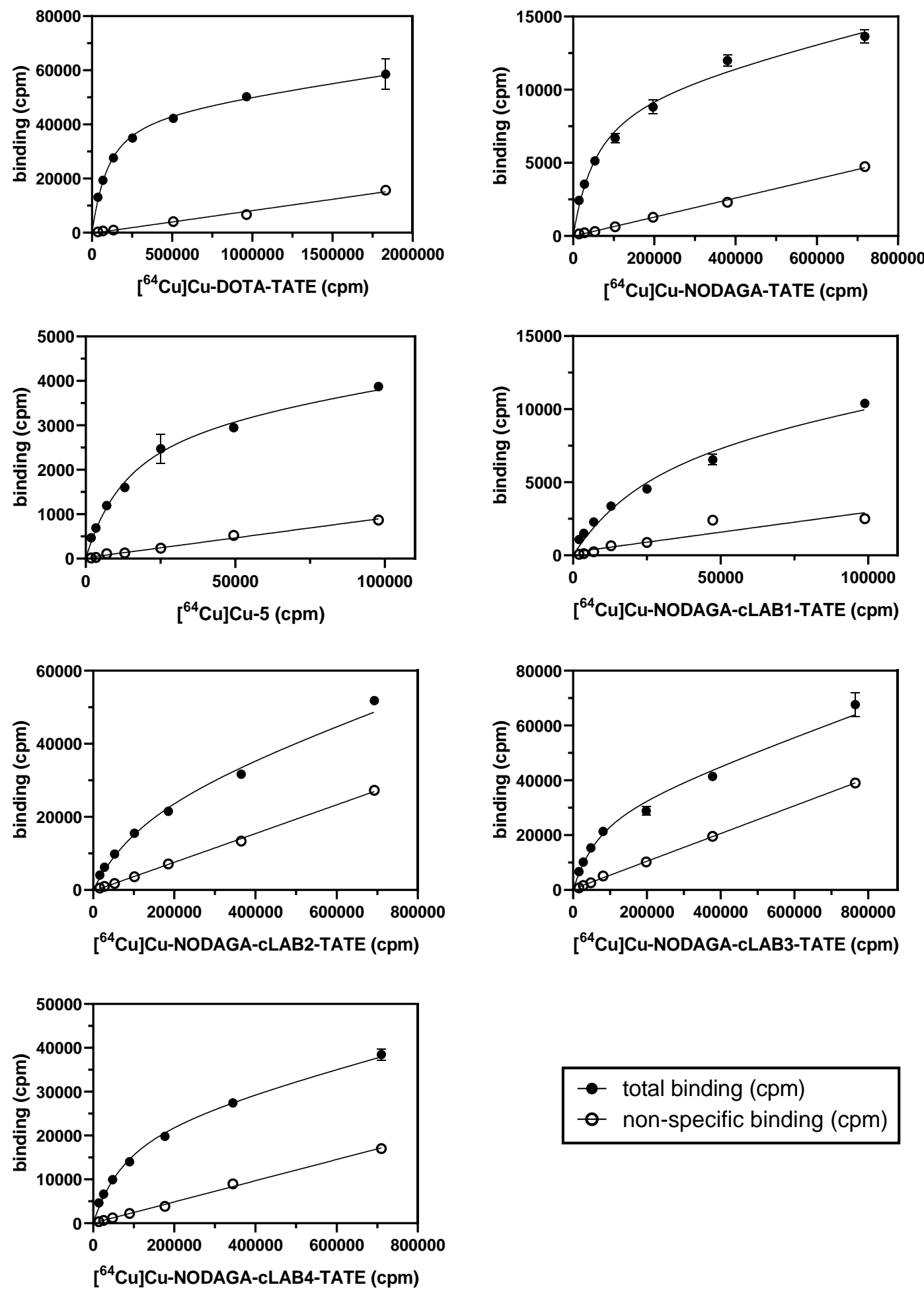

-- total binding (cpm)

- non-specific binding (cpm) 


\section{Figure S6: Non-specific binding curves}

Binding curves of the different ${ }^{64} \mathrm{Cu}$-labeled TATE derivatives using MPC cell lysates in the presence of $1 \mu \mathrm{M}$ DOTA-TATE with linear regressions and the obtained slopes and coefficients of determination. The slopes were used for the term 'NS' within the model of 'One site - Total, accounting for ligand depletion', which was used for analysis of the total binding curves (see Figure S5).
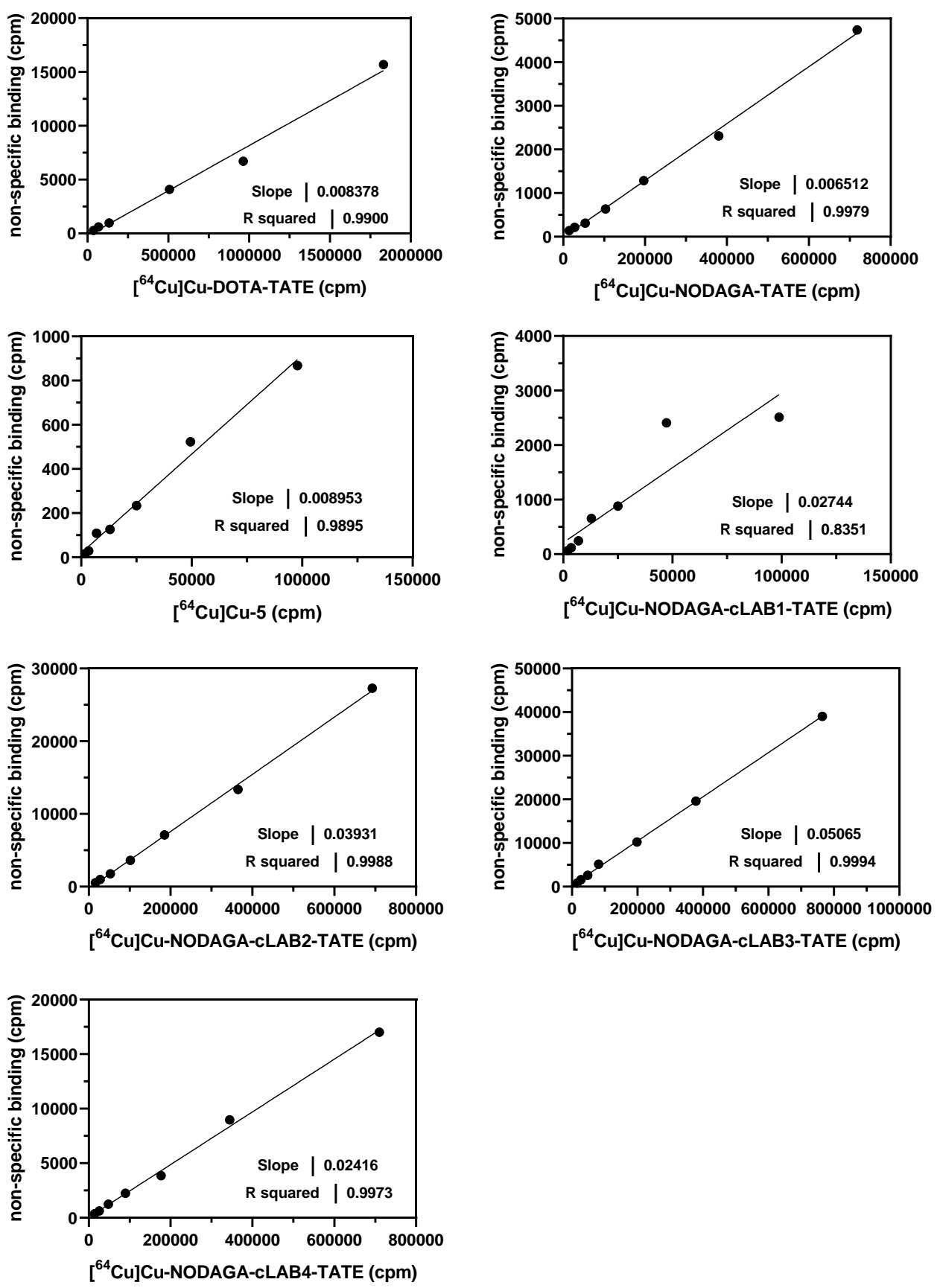


\section{Figure S7: Calibration curves}

Plots of 'radioactivity' $=\mathrm{f}$ (amount of radioligand) with linear regressions. The derived slopes served as values for 'SpecAct' within the model of 'One site - Total, accounting for ligand depletion', which was used for analysis of the total binding curves (see Figure S5).
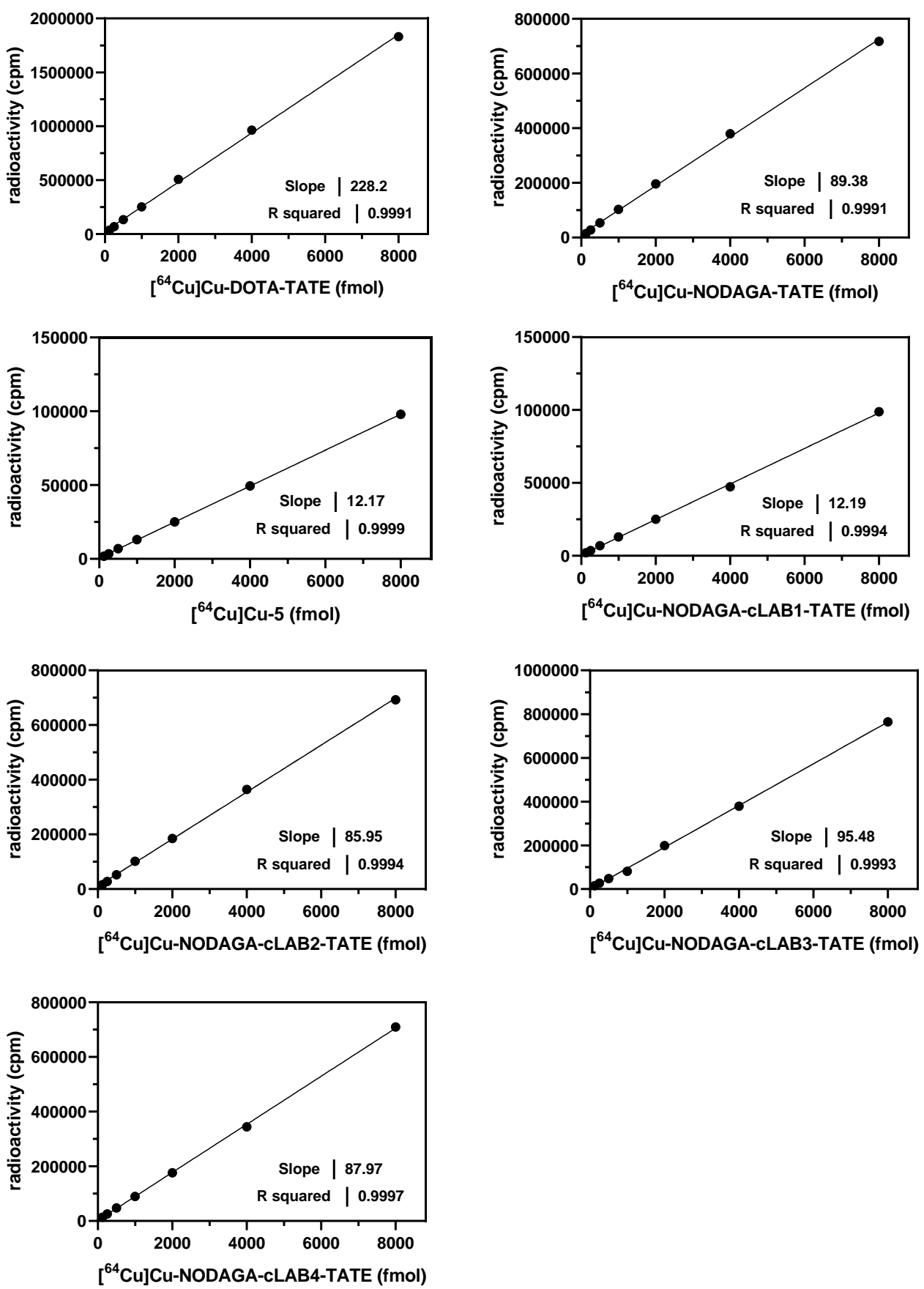


\section{Figure S8: Plasma stability}

Radioactivity-detected HPLC chromatograms of the different ${ }^{64} \mathrm{Cu}$-labeled TATEs after incubation in mouse and/or human plasma after $24 \mathrm{~h}$. In each chromatogram, the different traces are stacked $(x=+0.5$ min; $y=+10)$.
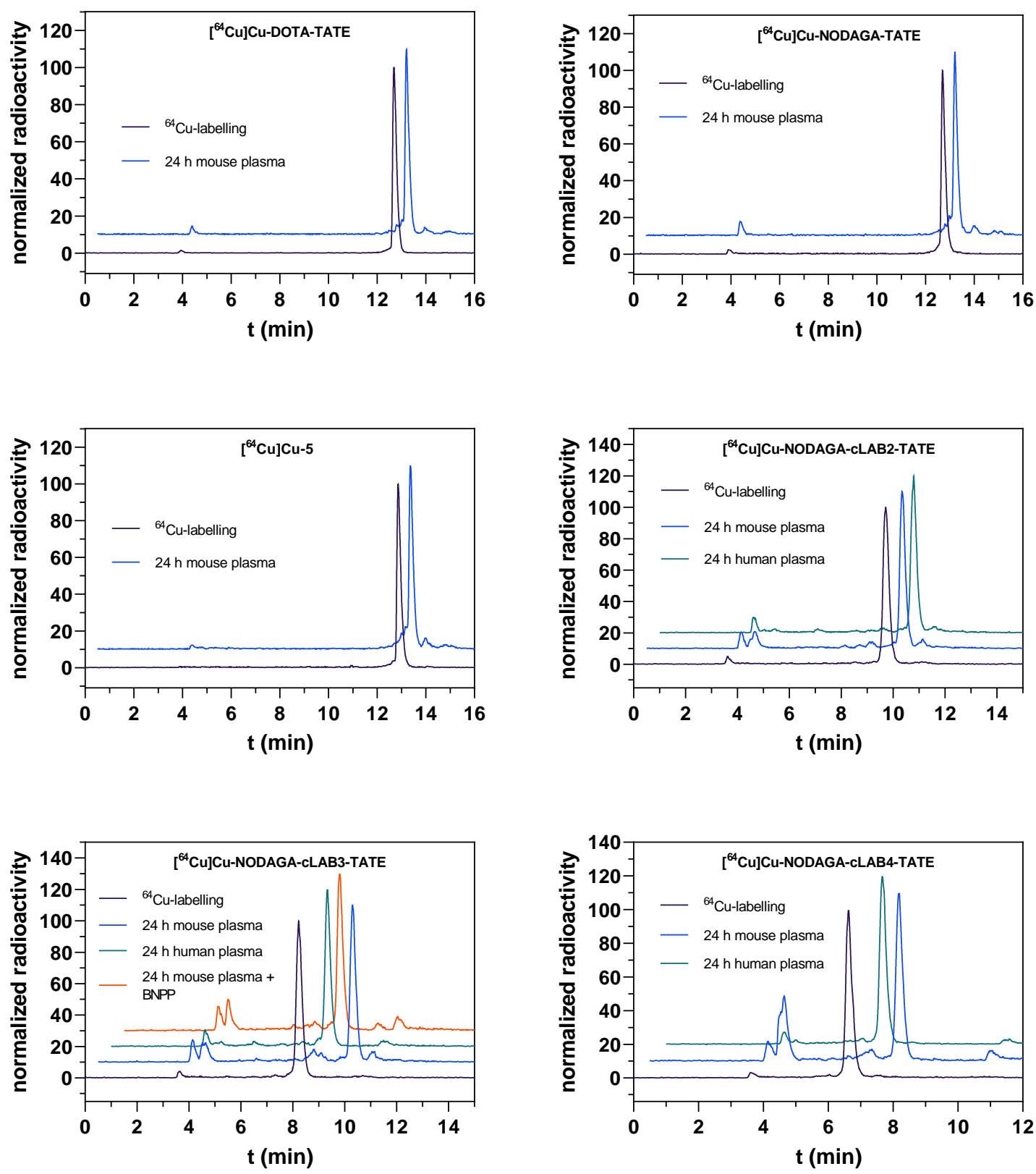
HPLC-DAD-HRMS chromatograms (top TIC scan, bottom $254 \mathrm{~nm}$ ) of a mixture of NODAGAcLAB2-TATE $\left(t_{R}=5.9 \mathrm{~min}\right.$ TIC scan) and NODAGA-cLAB3-TATE ( $t_{R}=6.3 \mathrm{~min}$ TIC scan)
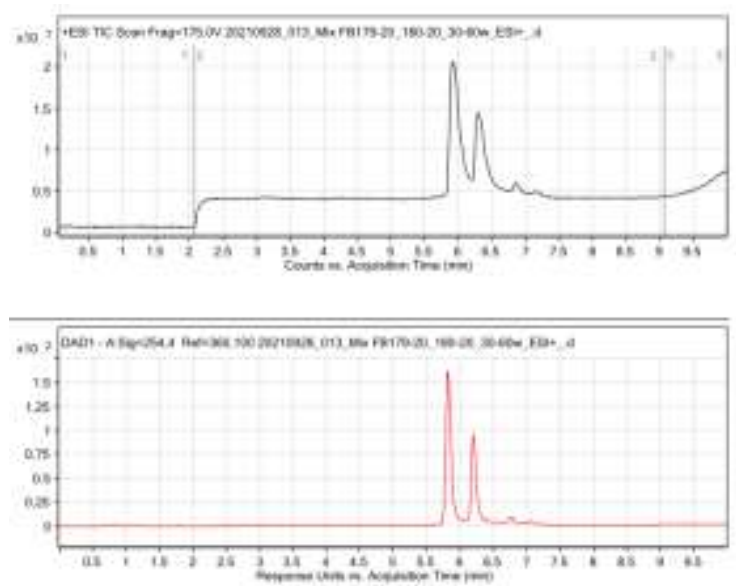

HPLC-HRMS chromatogram (TIC scan) of NODAGA-cLAB3-TATE after incubation for $24 \mathrm{~h}$ in mouse plasma at $37^{\circ} \mathrm{C}$. Mass detection was started at $2.1 \mathrm{~min}$. The two peaks were identified as NODAGA-cLAB3-TATE $\left(t_{R}=6.09 \mathrm{~min}\right)$ and NODAGA-cLAB2-TATE $\left(t_{R}=6.38 \mathrm{~min}\right)$ based on the respective mass spectra (bottom).
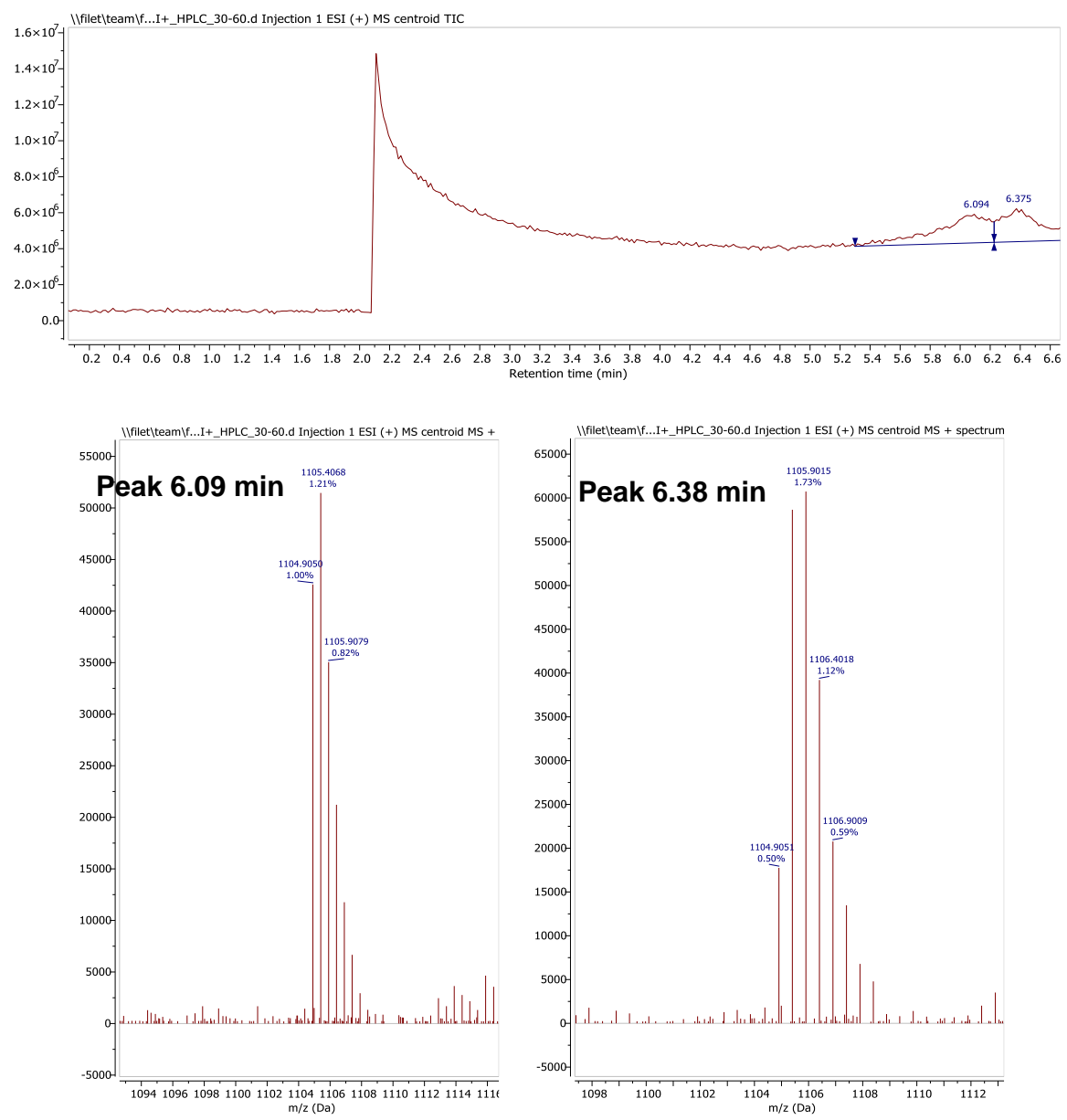
Figure S9: Time-dependent SUV data of muscle and liver for the different ${ }^{64} \mathrm{Cu}$-labeled TATE derivatives and the isolated albumin binder

Standard uptake values (SUV, decay-corrected) as a function of time (up to $48 \mathrm{~h}$ ) obtained by PET acquisition (0-2 $\mathrm{h}$ in dynamic mode and time points 5, 24 and $48 \mathrm{~h}$ p.i.; the $\mathrm{x}$-axes are segmented accordingly). Data shown are mean values of a group of MPC tumor bearing NMRI$\mathrm{nu} / \mathrm{nu}$ mice $(\mathrm{n}=2-3)$. The area under the respective curves were filled with color for a better visualization (inspired by graphs in Benesova et al. ${ }^{14}$. 
Muscle Liver
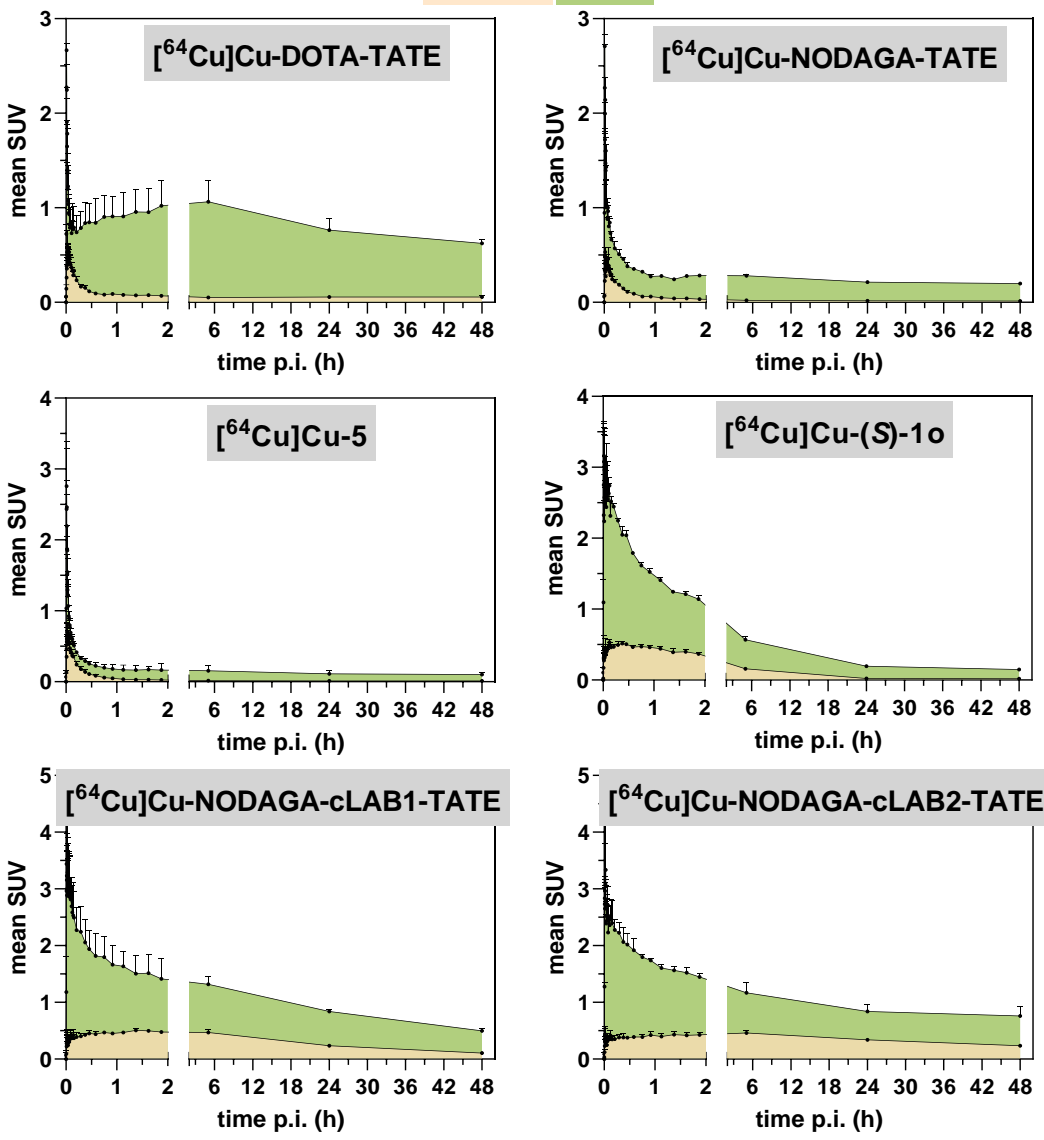

${ }^{5}\left[{ }^{64} \mathrm{Cu}\right] \mathrm{Cu}-\mathrm{NODAGA-cLAB} 3-\mathrm{TATE}$

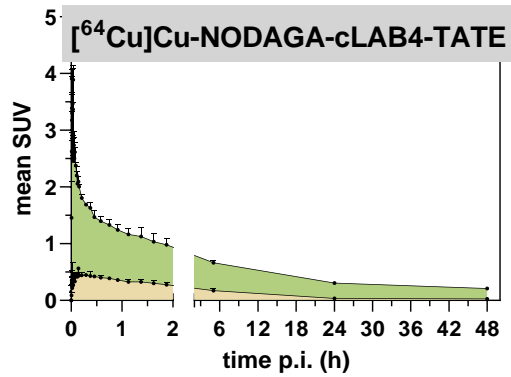




\section{Figure S10: Association experiments for $\left[{ }^{64} \mathrm{Cu}\right] \mathrm{Cu}-\mathrm{NODAGA-TATE}$ (Left column) and $\left[{ }^{64} \mathrm{Cu}\right] \mathrm{Cu}-5$ (right column)}

Plots of 'binding' $=\mathrm{f}($ time) for total binding (top) and non-specific binding (middle, in the presence of $1 \mu \mathrm{M}$ of the respective non-radioactive peptide) of the radioligands at different concentrations $(0.625,2.5$ and $20 \mathrm{nM})$ towards MPC cell lysates expressing $\mathrm{SST}_{2}$. Data shown are mean values $( \pm S D)$ of one experiment, which was performed in duplicate. Binding data were corrected for filter binding. Radioligand binding was 16 and $4 \%$ at $0.625 \mathrm{nM}$ for $\left[{ }^{64} \mathrm{Cu}\right] \mathrm{Cu}$ NODAGA-TATE and $\left[{ }^{64} \mathrm{Cu}\right] \mathrm{Cu}-5$, respectively. Therefore, radioligand depletion was not considered in these experiments. Total binding curves were analysed using the model of 'Onephase association' as implemented in GraphPad Prism (robust regression; non-specific binding, $Y_{0}$, was not constrained as the fitted values were largely in accordance to the determined values (middle)). The derived $k_{\text {obs }}$ values were plotted against [radioligand] (Bottom) and analysis was performed using equation SII (see Discussion S3). The obtained microscopic rate constants are summarized in Table S3.
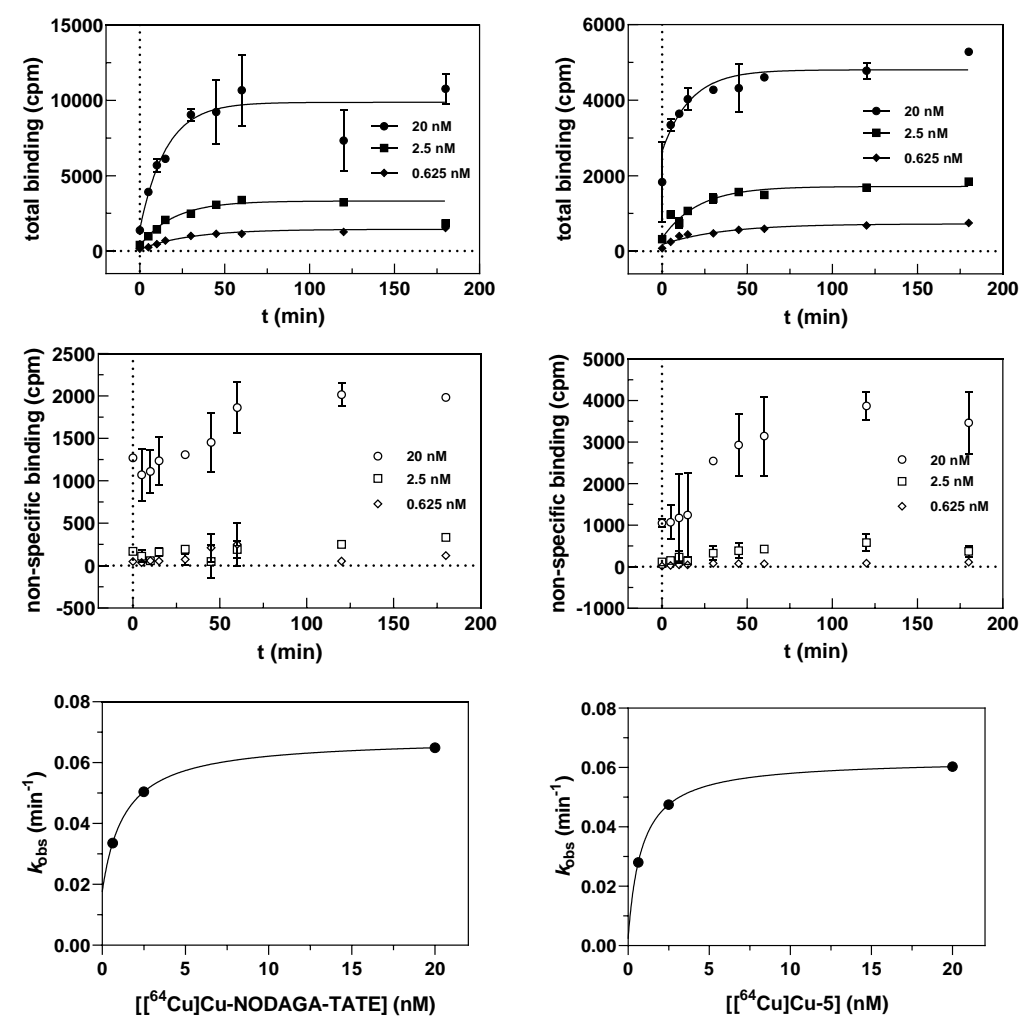


\section{Figure S11: Dissociation experiments für $\left[{ }^{64} \mathrm{Cu}\right] \mathrm{Cu}-\mathrm{NODAGA-TATE}$, $\left[{ }^{64} \mathrm{Cu}\right] \mathrm{Cu}-5$ and $\left[{ }^{64} \mathrm{Cu}\right] \mathrm{Cu}-\mathrm{NODAGA}-\mathrm{CLAB} 1-\mathrm{TATE}$}

Plots of 'total binding' $=f($ time $)$ with nonlinear regressions using the preferred model obtained by comparison of 'One phase' or 'Two phase exponential decay' as implemented in GraphPad Prism. MPC cell lysates were incubated with $20 \mathrm{nM}$ radioligand for $1 \mathrm{~h}$ and dissociation was started by the addition of $20 \mu \mathrm{M}$ of the respective non-radioactive peptide. Data shown are mean values $( \pm S D)$ of one experiment, which was performed in triplicate. Data were corrected for filter binding. Dotted lines indicate non-specific binding, which was determined separately ( $1 \mathrm{~h}$ incubation period in the presence of $1 \mu \mathrm{M}$ of the respective non-radioactive peptide). The obtained $k_{\text {off }}$ values are summarized in Table S3.
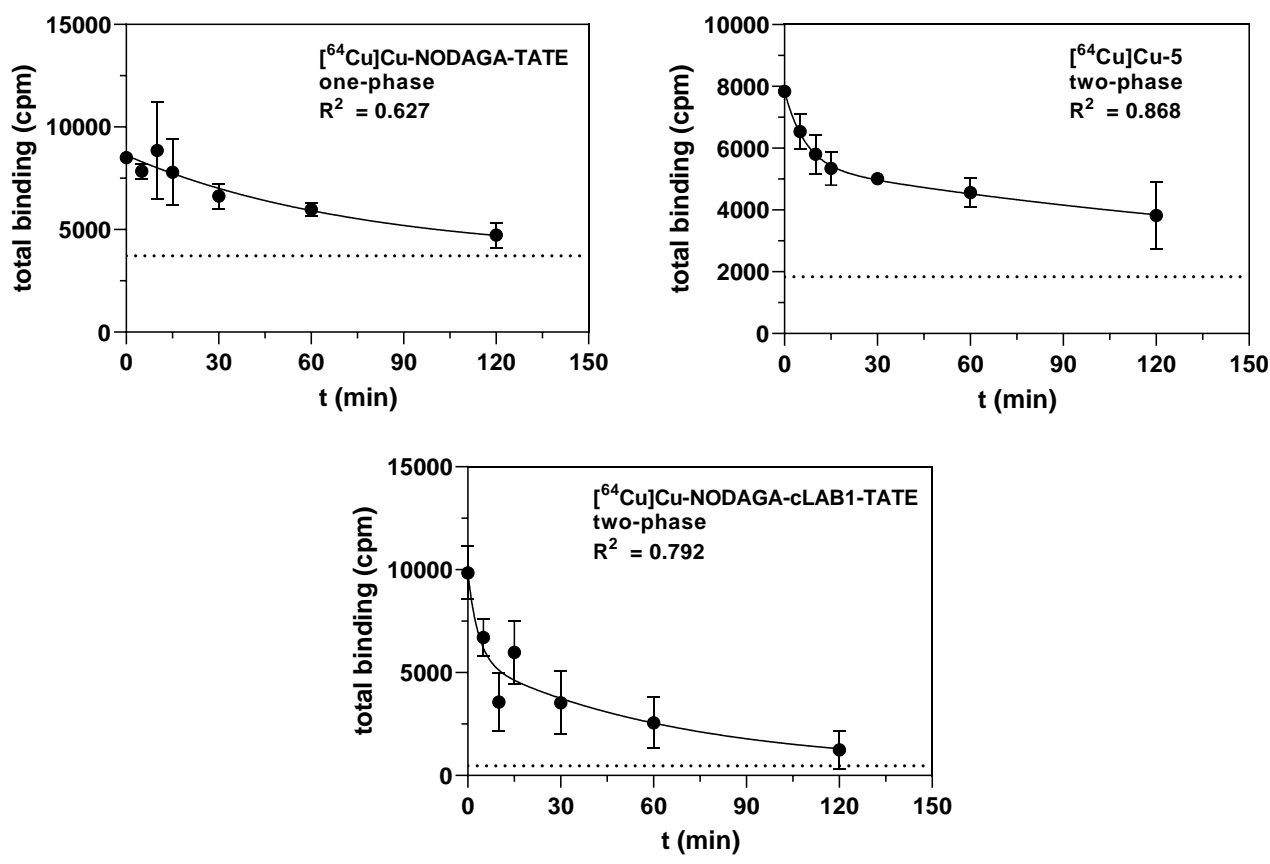
Table S1: SUV data for the different ${ }^{64} \mathrm{Cu}$-labeled TATE derivatives and the isolated albumin binder $\left[{ }^{64} \mathrm{Cu}\right]$ Cu-NODAGA-TATE

\begin{tabular}{l|cc|cc|cc|cc|cc|cc} 
Time (h) & \multicolumn{2}{|c|}{ Heart } & \multicolumn{2}{|c|}{ Kidney } & \multicolumn{2}{c|}{ Liver } & \multicolumn{2}{c|}{ Muscle } & \multicolumn{2}{c}{ MPC tumor } & \multicolumn{2}{c}{ Vena Cava } \\
\hline 0.001 & 0 & 0 & 0 & 0 & 0 & 0 & 0 & 0 & 0 & 0 & 0 & 0 \\
0.004 & 0.758 & 0.767 & 0.08 & 0.2055 & 0.031 & 0.087 & 0.001 & 0.008 & 0.001 & 0.004 & 8.019 & 7.71 \\
0.007 & 4.263 & 3.096 & 1.514 & 1.7605 & 0.734 & 1.159 & 0.075 & 0.069 & 0.219 & 0.33 & 98.842 & 73.607 \\
0.01 & 8.404 & 7.524 & 3.646 & 3.6885 & 1.946 & 2.593 & 0.32 & 0.13 & 0.971 & 0.693 & 33.652 & 23.707 \\
0.013 & 15.641 & 9.452 & 4.806 & 4.8825 & 2.618 & 2.798 & 0.347 & 0.337 & 1.416 & 1.112 & 3.897 & 2.629 \\
0.015 & 10.885 & 9.562 & 5.453 & 5.4225 & 2.083 & 1.907 & 0.293 & 0.531 & 1.388 & 1.667 & 1.985 & 2.899 \\
0.018 & 6.26 & 7.903 & 6.017 & 5.932 & 1.641 & 1.802 & 0.318 & 0.391 & 1.821 & 2.144 & 1.618 & 1.603 \\
0.021 & 7.839 & 5.379 & 6.5975 & 6.7435 & 1.971 & 2.309 & 0.538 & 0.527 & 2.332 & 2.291 & 0.288 & 2.031 \\
0.024 & 5.143 & 5.163 & 7.071 & 6.947 & 1.745 & 1.453 & 0.38 & 0.434 & 2.297 & 2.485 & 1.644 & 1.93 \\
0.026 & 6.678 & 4.273 & 8.8035 & 8.2565 & 1.247 & 1.696 & 0.343 & 0.302 & 2.902 & 2.909 & 2.998 & 1.727 \\
0.029 & 2.487 & 2.101 & 9.384 & 8.517 & 1.355 & 1.431 & 0.262 & 0.286 & 3.019 & 2.744 & 1.017 & 1.966 \\
0.032 & 2.075 & 4.627 & 9.3755 & 8.8565 & 1.502 & 1.707 & 0.368 & 0.487 & 2.72 & 2.672 & 1.278 & 0.783 \\
0.035 & 2.598 & 1.812 & 9.7695 & 8.9205 & 1.214 & 0.793 & 0.444 & 0.473 & 2.961 & 2.854 & 1.281 & 1.684 \\
0.038 & 2.036 & 2.197 & 10.1715 & 9.3565 & 1.598 & 1.263 & 0.31 & 0.47 & 2.635 & 3.177 & 0.835 & 1.255 \\
0.04 & 2.75 & 2.152 & 10.39 & 9.5755 & 0.907 & 1.228 & 0.467 & 0.452 & 3.582 & 3.269 & 0.761 & 0.386 \\
0.046 & 1.914 & 2.272 & 8.8145 & 8.677 & 1.036 & 1.023 & 0.38 & 0.463 & 3.535 & 3.423 & 1.944 & 0.667 \\
0.054 & 1.958 & 1.908 & 9.2875 & 9.143 & 0.959 & 1.044 & 0.3 & 0.468 & 3.674 & 3.998 & 1.262 & 0.97 \\
0.063 & 2.27 & 1.821 & 9.69 & 9.6015 & 0.871 & 0.892 & 0.364 & 0.436 & 3.862 & 4.148 & 0.758 & 1.256
\end{tabular}




\begin{tabular}{|c|c|c|c|c|c|c|c|c|c|c|c|c|}
\hline Time (h) & \multicolumn{2}{|c|}{ Heart } & \multicolumn{2}{|c|}{ Kidney } & \multicolumn{2}{|c|}{ Liver } & \multicolumn{2}{|c|}{ Muscle } & \multicolumn{2}{|c|}{ MPC tumor } & \multicolumn{2}{|c|}{ Vena Cava } \\
\hline 0.071 & 1.411 & 1.476 & 9.9165 & 10.0415 & 0.931 & 1.072 & 0.334 & 0.359 & 4.185 & 4.235 & 0.513 & 0.315 \\
\hline 0.079 & 1.57 & 1.9 & 10.1525 & 10.591 & 0.922 & 1.006 & 0.371 & 0.546 & 4.661 & 4.904 & 1.007 & 0.868 \\
\hline 0.092 & 1.444 & 1.099 & 10.378 & 11.0045 & 0.733 & 0.875 & 0.322 & 0.449 & 4.858 & 5.166 & 0.814 & 0.618 \\
\hline 0.108 & 1.329 & 1.269 & 11.181 & 11.8295 & 0.813 & 0.875 & 0.294 & 0.346 & 5.099 & 5.424 & 0.599 & 0.472 \\
\hline 0.125 & 0.966 & 0.888 & 12.0255 & 12.165 & 0.733 & 0.728 & 0.238 & 0.332 & 5.551 & 5.679 & 0.568 & 0.601 \\
\hline 0.142 & 1.137 & 0.759 & 12.262 & 12.625 & 0.6 & 0.726 & 0.292 & 0.298 & 5.908 & 6.338 & 0.596 & 0.711 \\
\hline 0.158 & 0.762 & 0.68 & 12.989 & 13.2015 & 0.656 & 0.676 & 0.222 & 0.267 & 6.259 & 6.402 & 0.888 & 0.492 \\
\hline 0.208 & 0.839 & 0.847 & 13.476 & 13.2845 & 0.518 & 0.625 & 0.207 & 0.234 & 6.437 & 6.77 & 0.467 & 0.376 \\
\hline 0.292 & 0.514 & 0.582 & 13.9035 & 13.827 & 0.473 & 0.543 & 0.187 & 0.184 & 7.55 & 7.644 & 0.438 & 0.361 \\
\hline 0.375 & 0.479 & 0.414 & 13.659 & 13.201 & 0.447 & 0.472 & 0.147 & 0.145 & 8.058 & 8.386 & 0.281 & 0.343 \\
\hline 0.458 & 0.452 & 0.269 & 12.7735 & 12.2535 & 0.408 & 0.354 & 0.122 & 0.099 & 8.426 & 9.033 & 0.237 & 0.243 \\
\hline 0.583 & 0.316 & 0.272 & 11.488 & 10.751 & 0.356 & 0.353 & 0.092 & 0.088 & 9.141 & 9.133 & 0.28 & 0.205 \\
\hline 0.75 & 0.194 & 0.191 & 9.6115 & 8.684 & 0.329 & 0.318 & 0.061 & 0.063 & 9.381 & 9.399 & 0.23 & 0.182 \\
\hline 0.917 & 0.165 & 0.167 & 8.0685 & 7.1 & 0.263 & 0.286 & 0.057 & 0.064 & 9.537 & 9.571 & 0.141 & 0.126 \\
\hline 1.125 & 0.134 & 0.145 & 6.4135 & 5.4365 & 0.276 & 0.28 & 0.053 & 0.043 & 9.521 & 9.426 & 0.157 & 0.138 \\
\hline 1.375 & 0.099 & 0.088 & 4.961 & 4.044 & 0.247 & 0.241 & 0.036 & 0.045 & 9.533 & 9.38 & 0.112 & 0.115 \\
\hline 1.625 & 0.085 & 0.109 & 3.9915 & 3.2005 & 0.276 & 0.283 & 0.039 & 0.042 & 9.478 & 9.301 & 0.094 & 0.137 \\
\hline 1.875 & 0.11 & 0.084 & 3.2475 & 2.5905 & 0.292 & 0.275 & 0.033 & 0.036 & 9.401 & 9.109 & 0.105 & 0.142 \\
\hline 5 & 0.104 & 0.061 & 0.9465 & 0.6995 & 0.268 & 0.292 & 0.02 & 0.02 & 6.97 & 6.537 & & \\
\hline 24 & 0.104 & 0.06 & 0.2825 & 0.216 & 0.221 & 0.205 & 0.015 & 0.015 & 2.104 & 1.974 & & \\
\hline 48 & 0.059 & 0.065 & 0.207 & 0.173 & 0.201 & 0.196 & 0.014 & 0.013 & 1.501 & 1.293 & & \\
\hline
\end{tabular}




\section{$\left[{ }^{64} \mathrm{Cu}\right] \mathrm{Cu}$-DOTA-TATE}

\begin{tabular}{c|cc|cc|cc|cc|cc|cc} 
Time (h) & \multicolumn{2}{|c|}{ Heart } & \multicolumn{2}{|c|}{ Kidney } & \multicolumn{2}{|c|}{ Liver } & \multicolumn{2}{c|}{ Muscle } & \multicolumn{2}{|c}{ MPC tumor } & \multicolumn{2}{c}{ Vena Cava } \\
\hline 0.001 & 0 & 0 & 0 & 0 & 0 & 0 & 0 & 0 & 0 & 0 & 0 & 0 \\
0.004 & 1.863 & 1.748 & 0.336 & 0.3065 & 0.05 & 0.066 & 0.068 & 0.053 & 0.025 & 0.052 & 6.15 & 14.849 \\
0.007 & 8.498 & 5.055 & 2.2855 & 2.446 & 0.791 & 0.658 & 0.112 & 0.174 & 0.365 & 0.132 & 69.832 & 52.982 \\
0.01 & 6.265 & 5.804 & 5.0145 & 5.38 & 2.445 & 2.102 & 0.271 & 0.253 & 0.819 & 0.416 & 21.759 & 37.7 \\
0.013 & 4.748 & 5.586 & 7.323 & 7.68 & 2.62 & 2.714 & 0.45 & 0.316 & 1.427 & 0.666 & 0.772 & 1.451 \\
0.015 & 4.978 & 5.867 & 9.2435 & 9.549 & 2.229 & 2.265 & 0.363 & 0.396 & 1.92 & 1.055 & 4.401 & 0.32 \\
0.018 & 4.095 & 5.126 & 10.927 & 11.0885 & 2.083 & 1.732 & 0.293 & 0.417 & 2.467 & 1.698 & 2.304 & 2.663 \\
0.021 & 4.196 & 5.747 & 12.4365 & 12.5365 & 1.456 & 1.841 & 0.535 & 0.52 & 2.459 & 1.526 & 1.202 & 2.221 \\
0.024 & 4.407 & 2.734 & 13.3625 & 14.569 & 1.711 & 1.856 & 0.522 & 0.529 & 2.649 & 1.969 & 1.878 & 2.793 \\
0.026 & 3.054 & 2.772 & 13.744 & 14.8935 & 1.346 & 1.453 & 0.504 & 0.718 & 2.528 & 1.362 & 4.522 & 4.827 \\
0.029 & 2.486 & 2.383 & 12.1945 & 14.2985 & 1.268 & 1.52 & 0.496 & 0.601 & 3.188 & 1.508 & 1.678 & 1.955 \\
0.032 & 3.543 & 2.819 & 11.776 & 13.2995 & 1.046 & 1.7 & 0.548 & 0.603 & 3.009 & 1.831 & 3.013 & 0.073 \\
0.035 & 2.875 & 2.078 & 9.896 & 11.7235 & 1.058 & 1.337 & 0.56 & 0.4 & 3.656 & 2 & 1.462 & 0.498 \\
0.038 & 2.148 & 2.694 & 9.3425 & 10.6135 & 1.132 & 1.32 & 0.383 & 0.542 & 3.86 & 2.185 & 2.863 & 0.707 \\
0.04 & 1.871 & 2.414 & 8.64 & 9.912 & 0.808 & 1.337 & 0.391 & 0.45 & 3.49 & 2.462 & 0.793 & 1.12 \\
0.046 & 1.448 & 2.326 & 7.436 & 8.323 & 0.93 & 1.149 & 0.56 & 0.48 & 3.783 & 2.446 & 2.235 & 1.694 \\
0.054 & 1.808 & 2.098 & 6.4035 & 6.9835 & 0.808 & 1.085 & 0.437 & 0.366 & 4.452 & 3.033 & 1.397 & 1.109 \\
0.063 & 1.404 & 1.734 & 5.442 & 6.003 & 0.813 & 1.05 & 0.548 & 0.513 & 4.624 & 2.942 & 0.776 & 0.709 \\
0.071 & 1.275 & 1.573 & 4.8175 & 5.36 & 0.808 & 0.831 & 0.496 & 0.449 & 4.525 & 3.071 & 0.459 & 0.834 \\
0.079 & 1.101 & 1.347 & 4.42 & 4.7975 & 0.651 & 0.928 & 0.473 & 0.366 & 4.776 & 3.249 & 0.979 & 0.636
\end{tabular}




\begin{tabular}{|c|c|c|c|c|c|c|c|c|c|c|c|c|}
\hline Time (h) & \multicolumn{2}{|c|}{ Heart } & \multicolumn{2}{|c|}{ Kidney } & \multicolumn{2}{|c|}{ Liver } & \multicolumn{2}{|c|}{ Muscle } & \multicolumn{2}{|c|}{ MPC tumor } & \multicolumn{2}{|c|}{ Vena Cava } \\
\hline 0.092 & 1.451 & 1.104 & 3.975 & 4.0155 & 0.741 & 0.954 & 0.449 & 0.346 & 5.117 & 3.485 & 0.759 & 0.356 \\
\hline 0.108 & 1.525 & 0.871 & 3.2365 & 3.5815 & 0.641 & 0.824 & 0.407 & 0.327 & 5.45 & 3.582 & 0.87 & 0.501 \\
\hline 0.125 & 0.988 & 0.903 & 2.905 & 2.975 & 0.689 & 0.981 & 0.358 & 0.31 & 5.768 & 3.909 & 0.277 & 0.478 \\
\hline 0.142 & 0.81 & 0.714 & 2.61 & 2.691 & 0.713 & 0.831 & 0.312 & 0.263 & 6.118 & 4.416 & 0.404 & 0.244 \\
\hline 0.158 & 0.897 & 0.883 & 2.2605 & 2.4435 & 0.629 & 0.951 & 0.341 & 0.33 & 6.116 & 4.254 & 0.687 & 0.69 \\
\hline 0.208 & 0.587 & 0.534 & 1.736 & 1.786 & 0.611 & 0.871 & 0.258 & 0.21 & 6.477 & 4.486 & 0.312 & 0.349 \\
\hline 0.292 & 0.382 & 0.328 & 1.2415 & 1.3255 & 0.665 & 0.906 & 0.186 & 0.149 & 7.019 & 4.985 & 0.262 & 0.224 \\
\hline 0.375 & 0.32 & 0.276 & 0.9155 & 0.978 & 0.687 & 0.989 & 0.174 & 0.134 & 7.406 & 5.438 & 0.13 & 0.204 \\
\hline 0.458 & 0.243 & 0.225 & 0.8135 & 0.8935 & 0.706 & 0.984 & 0.12 & 0.111 & 7.685 & 5.475 & 0.157 & 0.147 \\
\hline 0.583 & 0.196 & 0.214 & 0.594 & 0.722 & 0.662 & 1.022 & 0.094 & 0.092 & 7.414 & 5.55 & 0.087 & 0.151 \\
\hline 0.75 & 0.178 & 0.171 & 0.599 & 0.819 & 0.746 & 1.06 & 0.082 & 0.082 & 7.693 & 5.679 & 0.076 & 0.153 \\
\hline 0.917 & 0.14 & 0.183 & 0.6345 & 0.9725 & 0.756 & 1.059 & 0.08 & 0.095 & 7.59 & 5.502 & 0.085 & 0.146 \\
\hline 1.125 & 0.134 & 0.152 & 0.5505 & 0.8415 & 0.73 & 1.089 & 0.076 & 0.083 & 7.512 & 5.546 & 0.066 & 0.109 \\
\hline 1.375 & 0.138 & 0.148 & 0.4595 & 0.7485 & 0.784 & 1.128 & 0.066 & 0.082 & 7.507 & 5.706 & 0.063 & 0.126 \\
\hline 1.625 & 0.115 & 0.148 & 0.6235 & 0.624 & 0.774 & 1.133 & 0.068 & 0.082 & 7.43 & 5.723 & 0.053 & 0.132 \\
\hline 1.875 & 0.124 & 0.174 & 0.5475 & 0.691 & 0.83 & 1.21 & 0.064 & 0.073 & 7.363 & 5.482 & 0.061 & 0.164 \\
\hline 5 & 0.165 & 0.197 & 0.2625 & 0.2675 & 0.905 & 1.22 & 0.049 & 0.053 & 5.389 & 4.335 & & \\
\hline 24 & 0.149 & 0.181 & 0.2305 & 0.2825 & 0.677 & 0.85 & 0.063 & 0.048 & 3.104 & 2.922 & & \\
\hline 48 & 0.145 & 0.144 & 0.273 & 0.255 & 0.596 & 0.652 & 0.07 & 0.044 & 2.586 & 2.198 & & \\
\hline
\end{tabular}




\section{$\left[{ }^{64} \mathrm{Cu}\right] \mathrm{Cu}-5$}

\begin{tabular}{|c|c|c|c|c|c|c|c|c|c|c|c|c|c|c|c|c|c|}
\hline \multirow{2}{*}{$\begin{array}{c}\text { Time (h) } \\
0.001\end{array}$} & \multicolumn{3}{|c|}{ Heart } & \multicolumn{2}{|c|}{ Kidney } & \multicolumn{3}{|c|}{ Liver } & \multicolumn{3}{|c|}{ Muscle } & \multicolumn{3}{|c|}{ MPC tumor } & \multicolumn{3}{|c|}{ Vena Cava } \\
\hline & 0.000 & 0.000 & 0.000 & 0.000 & 0.000 & 0.000 & 0.000 & 0.000 & 0.000 & 0.000 & 0.000 & 0.000 & 0.000 & 0.000 & 0.000 & 0.000 & 0.000 \\
\hline 0.004 & 0.652 & 1.410 & 0.693 & 0.038 & 0.145 & 0.050 & 0.022 & 0.128 & 0.014 & 0.009 & 0.001 & 0.013 & 0.019 & 0.000 & 1.113 & 6.732 & 0.285 \\
\hline 0.007 & 5.446 & 4.731 & 4.700 & 1.356 & 1.596 & 0.738 & 0.786 & 1.579 & 0.098 & 0.140 & 0.156 & 0.118 & 0.132 & 0.226 & 42.635 & 25.985 & 54.302 \\
\hline 0.01 & 7.763 & 8.211 & 8.280 & 3.840 & 3.426 & 2.154 & 1.656 & 3.500 & 0.263 & 0.428 & 0.361 & 0.422 & 0.515 & 1.146 & 48.768 & 89.029 & 40.384 \\
\hline 0.013 & 12.566 & 8.466 & 6.830 & 5.205 & 5.040 & 2.192 & 2.852 & 3.235 & 0.405 & 0.444 & 0.533 & 0.940 & 0.853 & 1.924 & 3.267 & 5.174 & 2.936 \\
\hline 0.015 & 13.865 & 7.370 & 6.216 & 6.528 & 5.988 & 2.436 & 2.098 & 2.855 & 0.505 & 0.455 & 0.857 & 1.215 & 1.474 & 2.425 & 1.864 & 2.285 & 1.255 \\
\hline 0.018 & 9.494 & 7.449 & 7.057 & 7.649 & 7.106 & 2.451 & 1.682 & 2.450 & 0.475 & 0.610 & 0.612 & 1.587 & 1.493 & 2.925 & 4.002 & 0.686 & 0.658 \\
\hline 0.021 & 7.237 & 6.013 & 6.166 & 8.749 & 8.200 & 1.768 & 1.613 & 2.237 & 0.554 & 0.605 & 0.772 & 1.591 & 1.705 & 3.097 & 0.445 & 4.070 & 0.325 \\
\hline 0.024 & 4.855 & 5.490 & 3.798 & 9.904 & 9.368 & 1.833 & 1.660 & 2.066 & 0.692 & 0.568 & 0.841 & 2.006 & 1.945 & 3.212 & 0.748 & 0.856 & 1.167 \\
\hline 0.026 & 5.086 & 4.029 & 7.291 & 12.322 & 11.726 & 1.376 & 1.329 & 1.842 & 0.471 & 0.404 & 0.689 & 1.977 & 1.935 & 3.201 & 1.642 & 3.117 & 2.060 \\
\hline 0.029 & 3.161 & 3.040 & 6.060 & 13.204 & 12.533 & 1.601 & 1.335 & 1.697 & 0.664 & 0.534 & 0.696 & 1.763 & 1.778 & 3.576 & 0.633 & 0.787 & 0.608 \\
\hline 0.032 & 4.048 & 3.092 & 5.657 & 14.436 & 13.240 & 1.384 & 0.991 & 1.506 & 0.598 & 0.613 & 0.587 & 2.270 & 2.080 & 3.378 & 0.477 & 3.288 & 1.536 \\
\hline 0.035 & 2.609 & 3.178 & 4.389 & 15.100 & 12.816 & 1.219 & 1.051 & 1.363 & 0.412 & 0.564 & 0.651 & 2.324 & 2.056 & 3.566 & 0.268 & 0.770 & 0.768 \\
\hline 0.038 & 3.483 & 3.603 & 4.299 & 14.937 & 12.959 & 1.273 & 0.983 & 1.408 & 0.481 & 0.594 & 0.730 & 2.227 & 2.425 & 3.789 & 1.591 & 0.670 & 0.294 \\
\hline 0.04 & 2.498 & 2.779 & 4.485 & 14.794 & 12.781 & 1.099 & 1.178 & 1.352 & 0.656 & 0.581 & 0.608 & 2.496 & 2.216 & 4.022 & 0.512 & 1.913 & 1.337 \\
\hline 0.046 & 2.424 & 2.289 & 2.539 & 12.853 & 10.281 & 1.014 & 0.966 & 1.225 & 0.645 & 0.620 & 0.666 & 2.829 & 2.427 & 4.344 & 0.875 & 1.254 & 2.607 \\
\hline 0.054 & 1.993 & 2.257 & 1.882 & 11.688 & 9.339 & 0.921 & 0.769 & 1.084 & 0.537 & 0.605 & 0.537 & 2.760 & 2.760 & 4.367 & 1.314 & 0.566 & 1.462 \\
\hline 0.063 & 1.755 & 1.888 & 1.823 & 10.914 & 8.523 & 0.824 & 0.855 & 0.999 & 0.466 & 0.554 & 0.596 & 2.985 & 2.774 & 4.773 & 0.600 & 0.959 & 1.541 \\
\hline 0.071 & 1.640 & 1.859 & 1.594 & 10.246 & 7.938 & 0.789 & 0.744 & 0.862 & 0.457 & 0.600 & 0.586 & 3.409 & 2.983 & 4.727 & 0.338 & 1.735 & 1.564 \\
\hline 0.079 & 1.237 & 1.838 & 1.630 & 9.431 & 7.468 & 0.821 & 0.616 & 0.862 & 0.512 & 0.424 & 0.484 & 3.371 & 3.094 & 5.132 & 2.036 & 0.962 & 2.107 \\
\hline
\end{tabular}




\begin{tabular}{|c|c|c|c|c|c|c|c|c|c|c|c|c|c|c|c|c|c|}
\hline Time (h) & & Heart & & $\mathbf{K i}$ & & & Liver & & & Muscle & & & PC tum & & & ena $\mathrm{Ca}$ & \\
\hline 0.092 & 1.461 & 1.560 & 1.215 & 8.770 & 6.873 & 0.727 & 0.623 & 0.728 & 0.425 & 0.435 & 0.425 & 3.620 & 3.439 & 5.197 & 0.332 & 0.854 & 1.416 \\
\hline 0.108 & 1.196 & 1.431 & 1.121 & 7.953 & 6.289 & 0.614 & 0.594 & 0.673 & 0.448 & 0.414 & 0.349 & 3.910 & 3.459 & 5.443 & 0.300 & 0.983 & 1.535 \\
\hline 0.125 & 1.499 & 1.086 & 1.017 & 7.265 & 5.840 & 0.589 & 0.569 & 0.642 & 0.343 & 0.370 & 0.391 & 4.053 & 3.880 & 5.553 & 0.335 & 0.696 & 2.487 \\
\hline 0.142 & 1.183 & 1.153 & 0.808 & 6.947 & 5.530 & 0.550 & 0.509 & 0.561 & 0.358 & 0.367 & 0.336 & 4.234 & 4.077 & 5.599 & 0.399 & 0.799 & 0.775 \\
\hline 0.158 & 0.827 & 0.997 & 0.900 & 6.361 & 5.018 & 0.530 & 0.479 & 0.524 & 0.358 & 0.365 & 0.304 & 4.343 & 4.080 & 6.112 & 1.453 & 0.572 & 0.804 \\
\hline 0.208 & 0.775 & 0.880 & 0.569 & 5.191 & 4.361 & 0.404 & 0.424 & 0.420 & 0.251 & 0.283 & 0.195 & 4.890 & 4.506 & 5.902 & 0.207 & 0.532 & 1.153 \\
\hline 0.292 & 0.588 & 0.674 & 0.363 & 3.907 & 5.521 & 0.306 & 0.339 & 0.353 & 0.189 & 0.206 & 0.136 & 5.249 & 4.937 & 6.283 & 0.183 & 0.274 & 1.033 \\
\hline 0.375 & 0.402 & 0.510 & 0.284 & 3.028 & 5.168 & 0.263 & 0.292 & 0.324 & 0.130 & 0.171 & 0.112 & 5.503 & 4.936 & 6.459 & 0.167 & 0.162 & 1.054 \\
\hline 0.458 & 0.317 & 0.420 & 0.217 & 2.373 & 6.253 & 0.214 & 0.256 & 0.292 & 0.109 & 0.124 & 0.097 & 5.668 & 5.270 & 6.319 & 0.110 & 0.187 & 0.741 \\
\hline 0.583 & 0.211 & 0.306 & 0.146 & 2.076 & 5.768 & 0.197 & 0.212 & 0.268 & 0.076 & 0.110 & 0.068 & 5.703 & 5.309 & 6.432 & 0.105 & 0.161 & 0.309 \\
\hline 0.75 & 0.124 & 0.210 & 0.108 & 1.559 & 1.865 & 0.163 & 0.171 & 0.257 & 0.051 & 0.073 & 0.048 & 5.673 & 5.242 & 6.251 & 0.100 & 0.050 & 0.046 \\
\hline 0.917 & 0.099 & 0.154 & 0.088 & 1.209 & 1.184 & 0.136 & 0.173 & 0.241 & 0.039 & 0.056 & 0.048 & 5.407 & 5.010 & 6.006 & 0.035 & 0.042 & 0.026 \\
\hline 1.125 & 0.074 & 0.095 & 0.076 & 1.037 & 0.901 & 0.126 & 0.132 & 0.244 & 0.032 & 0.039 & 0.038 & 5.355 & 4.945 & 5.724 & 0.055 & 0.020 & 0.033 \\
\hline 1.375 & 0.051 & 0.069 & 0.066 & 0.744 & 0.723 & 0.121 & 0.140 & 0.232 & 0.026 & 0.036 & 0.028 & 5.101 & 4.637 & 5.374 & 0.027 & 0.021 & 0.026 \\
\hline 1.625 & 0.048 & 0.073 & 0.053 & 0.757 & 0.710 & 0.136 & 0.156 & 0.224 & 0.026 & 0.029 & 0.033 & 4.776 & 4.385 & 5.218 & 0.031 & 0.023 & 0.030 \\
\hline 1.875 & 0.043 & 0.057 & 0.062 & 0.674 & 0.655 & 0.086 & 0.152 & 0.256 & 0.019 & 0.024 & 0.033 & 4.512 & 4.119 & 4.966 & 0.031 & 0.014 & 0.034 \\
\hline 5 & 0.025 & 0.071 & 0.040 & 0.348 & 0.262 & 0.104 & 0.125 & 0.239 & 0.013 & 0.009 & 0.018 & 2.562 & 2.300 & 3.360 & & & \\
\hline 24 & 0.016 & 0.030 & 0.031 & 0.080 & 0.060 & 0.084 & 0.091 & 0.162 & 0.007 & 0.007 & 0.025 & 1.145 & 0.953 & 1.650 & & & \\
\hline 48 & 0.018 & 0.028 & 0.032 & 0.057 & 0.046 & 0.087 & 0.081 & 0.141 & 0.008 & 0.006 & 0.020 & 0.625 & 0.510 & 1.049 & & & \\
\hline
\end{tabular}




\section{$\left[{ }^{64} \mathrm{Cu}\right] \mathrm{Cu}-N O D A G A-c L A B 1-T A T E$}

\begin{tabular}{|c|c|c|c|c|c|c|c|c|c|c|c|c|c|c|c|c|c|c|}
\hline Time (h) & \multicolumn{3}{|c|}{ Heart } & \multicolumn{3}{|c|}{ Kidney } & \multicolumn{3}{|c|}{ Liver } & \multicolumn{3}{|c|}{ Muscle } & \multicolumn{3}{|c|}{ MPC tumor } & \multicolumn{3}{|c|}{ Vena Cava } \\
\hline 0.001 & 0.000 & 0.000 & 0.000 & 0.000 & 0.000 & 0.000 & 0.000 & 0.000 & 0.000 & 0.000 & 0.000 & 0.000 & 0.000 & 0.000 & 0.000 & 0.000 & 0.000 & 0.000 \\
\hline 0.004 & 0.996 & 0.581 & 0.163 & 0.039 & 0.022 & 0.015 & 0.035 & 0.038 & 0.126 & 0.001 & 0.002 & 0.003 & 0.001 & 0.002 & 0.000 & 6.651 & 0.045 & 0.763 \\
\hline 0.007 & 5.721 & 5.872 & 4.373 & 0.844 & 0.898 & 0.784 & 0.989 & 0.668 & 1.882 & 0.088 & 0.149 & 0.050 & 0.055 & 0.098 & 0.064 & 50.974 & 38.180 & 49.893 \\
\hline 0.01 & 8.982 & 9.126 & 8.440 & 2.259 & 2.219 & 2.465 & 2.382 & 2.179 & 4.332 & 0.169 & 0.326 & 0.169 & 0.216 & 0.436 & 0.324 & 80.614 & 31.833 & 57.729 \\
\hline 0.013 & 26.072 & 16.886 & 8.933 & 3.574 & 2.473 & 2.613 & 2.800 & 3.001 & 4.515 & 0.320 & 0.345 & 0.166 & 0.253 & 0.483 & 0.420 & 3.287 & 4.087 & 3.631 \\
\hline 0.015 & 12.027 & 20.501 & 7.439 & 3.129 & 3.335 & 3.073 & 2.863 & 2.760 & 3.850 & 0.340 & 0.448 & 0.319 & 0.445 & 0.687 & 0.451 & 5.295 & 5.962 & 3.485 \\
\hline 0.018 & 15.244 & 22.743 & 8.356 & 2.871 & 2.876 & 3.078 & 3.063 & 2.385 & 4.252 & 0.223 & 0.434 & 0.186 & 0.210 & 0.547 & 0.459 & 4.369 & 7.790 & 2.732 \\
\hline 0.021 & 16.093 & 13.341 & 8.367 & 3.830 & 3.260 & 3.602 & 2.393 & 2.490 & 3.770 & 0.265 & 0.392 & 0.200 & 0.372 & 0.661 & 0.407 & 7.312 & 8.738 & 4.943 \\
\hline 0.024 & 11.580 & 18.302 & 8.597 & 3.676 & 3.115 & 3.347 & 3.533 & 2.247 & 3.729 & 0.231 & 0.493 & 0.229 & 0.223 & 0.586 & 0.514 & 11.247 & 6.539 & 2.248 \\
\hline 0.026 & 13.865 & 14.617 & 8.637 & 5.345 & 3.792 & 4.347 & 2.603 & 2.627 & 3.786 & 0.239 & 0.521 & 0.355 & 0.234 & 0.662 & 0.537 & 11.345 & 3.967 & 4.243 \\
\hline 0.029 & 10.944 & 9.895 & 8.295 & 5.473 & 4.750 & 5.172 & 2.611 & 2.792 & 4.259 & 0.224 & 0.547 & 0.304 & 0.313 & 0.791 & 0.491 & 5.041 & 4.213 & 3.909 \\
\hline 0.032 & 11.205 & 8.705 & 6.715 & 5.217 & 4.777 & 4.793 & 3.263 & 2.685 & 3.926 & 0.313 & 0.513 & 0.248 & 0.244 & 0.664 & 0.506 & 8.326 & 3.724 & 2.430 \\
\hline 0.035 & 9.574 & 8.819 & 7.783 & 5.872 & 4.231 & 4.873 & 2.491 & 2.322 & 4.046 & 0.181 & 0.416 & 0.307 & 0.370 & 0.601 & 0.538 & 4.854 & 4.210 & 2.380 \\
\hline 0.038 & 9.513 & 9.196 & 6.726 & 5.433 & 4.865 & 4.775 & 3.175 & 2.322 & 3.747 & 0.203 & 0.448 & 0.221 & 0.497 & 0.897 & 0.604 & 5.353 & 3.814 & 4.040 \\
\hline 0.04 & 8.410 & 9.637 & 7.802 & 4.543 & 4.094 & 4.518 & 2.688 & 2.587 & 3.620 & 0.197 & 0.278 & 0.254 & 0.343 & 0.738 & 0.431 & 3.275 & 1.629 & 4.607 \\
\hline 0.046 & 7.843 & 10.070 & 7.823 & 3.776 & 2.982 & 3.338 & 2.552 & 2.472 & 3.601 & 0.230 & 0.424 & 0.334 & 0.496 & 0.937 & 0.739 & 5.825 & 3.845 & 6.549 \\
\hline 0.054 & 9.247 & 7.386 & 7.693 & 3.359 & 3.332 & 3.348 & 2.555 & 2.581 & 3.730 & 0.305 & 0.435 & 0.330 & 0.652 & 0.998 & 0.693 & 8.125 & 2.253 & 2.233 \\
\hline 0.063 & 7.857 & 9.215 & 7.318 & 3.530 & 2.923 & 3.108 & 2.672 & 2.380 & 3.749 & 0.270 & 0.351 & 0.291 & 0.545 & 1.062 & 0.769 & 3.651 & 2.472 & 2.713 \\
\hline 0.071 & 7.755 & 7.759 & 6.515 & 3.379 & 2.835 & 3.108 & 2.435 & 2.443 & 3.694 & 0.296 & 0.433 & 0.306 & 0.620 & 1.035 & 0.825 & 3.222 & 2.543 & 2.167 \\
\hline 0.079 & 8.123 & 7.556 & 7.363 & 3.208 & 3.146 & 2.986 & 2.606 & 2.356 & 3.665 & 0.269 & 0.414 & 0.326 & 0.630 & 1.048 & 0.808 & 3.830 & 3.103 & 2.680 \\
\hline 0.092 & 7.308 & 8.309 & 6.410 & 3.125 & 3.063 & 3.012 & 2.782 & 2.446 & 3.220 & 0.329 & 0.529 & 0.314 & 0.944 & 0.956 & 0.987 & 4.314 & 4.338 & 2.944 \\
\hline 0.108 & 8.168 & 8.330 & 6.755 & 3.255 & 2.952 & 2.945 & 2.525 & 2.300 & 3.261 & 0.301 & 0.482 & 0.337 & 0.962 & 1.336 & 1.103 & 5.046 & 3.208 & 2.420 \\
\hline 0.125 & 6.798 & 7.712 & 6.399 & 3.261 & 2.874 & 2.823 & 2.299 & 2.318 & 3.145 & 0.318 & 0.422 & 0.367 & 0.890 & 1.291 & 1.099 & 3.928 & 4.925 & 2.990 \\
\hline 0.142 & 7.671 & 8.827 & 5.940 & 3.237 & 2.682 & 2.765 & 2.170 & 2.223 & 3.195 & 0.361 & 0.535 & 0.369 & 1.142 & 1.511 & 1.284 & 4.203 & 3.183 & 3.083 \\
\hline
\end{tabular}




\begin{tabular}{|c|c|c|c|c|c|c|c|c|c|c|c|c|c|c|c|c|c|c|}
\hline \multirow{2}{*}{$\begin{array}{c}\text { Time (h) } \\
0.158\end{array}$} & \multicolumn{3}{|c|}{ Heart } & \multicolumn{3}{|c|}{ Kidney } & \multicolumn{3}{|c|}{ Liver } & \multicolumn{3}{|c|}{ Muscle } & \multicolumn{3}{|c|}{ MPC tumor } & \multicolumn{3}{|c|}{ Vena Cava } \\
\hline & 6.707 & 8.251 & 5.839 & 3.003 & 2.622 & 2.796 & 2.294 & 2.165 & 3.024 & 0.323 & 0.453 & 0.329 & 0.965 & 1.674 & 1.471 & 4.003 & 3.230 & 2.179 \\
\hline 0.208 & 7.107 & 6.936 & 5.492 & 2.911 & 2.669 & 2.484 & 2.066 & 2.026 & 2.727 & 0.362 & 0.485 & 0.327 & 1.241 & 1.864 & 1.537 & 3.762 & 2.407 & 2.035 \\
\hline 0.292 & 6.705 & 6.395 & 5.038 & 2.933 & 2.568 & 2.441 & 2.023 & 1.941 & 2.762 & 0.372 & 0.465 & 0.364 & 1.589 & 2.101 & 1.866 & 3.832 & 2.218 & 2.462 \\
\hline 0.375 & 5.816 & 6.218 & 4.913 & 2.616 & 2.325 & 2.305 & 1.921 & 1.723 & 2.521 & 0.402 & 0.461 & 0.419 & 1.894 & 2.343 & 2.136 & 3.514 & 1.953 & 1.956 \\
\hline 0.458 & 5.988 & 6.117 & 4.587 & 2.585 & 2.393 & 2.045 & 1.810 & 1.703 & 2.304 & 0.423 & 0.516 & 0.419 & 2.026 & 2.713 & 2.267 & 2.907 & 2.033 & 1.907 \\
\hline 0.583 & 5.642 & 5.848 & 4.341 & 2.301 & 2.121 & 2.033 & 1.682 & 1.512 & 2.257 & 0.413 & 0.488 & 0.420 & 2.297 & 3.162 & 2.541 & 2.757 & 1.573 & 1.910 \\
\hline 0.75 & 5.337 & 5.274 & 4.116 & 2.189 & 2.063 & 1.925 & 1.639 & 1.550 & 2.211 & 0.462 & 0.480 & 0.459 & 2.613 & 3.350 & 2.865 & 2.638 & 1.720 & 1.521 \\
\hline 0.917 & 4.731 & 5.095 & 3.824 & 1.905 & 1.908 & 1.788 & 1.504 & 1.424 & 2.064 & 0.428 & 0.461 & 0.462 & 2.740 & 3.750 & 3.006 & 2.098 & 1.303 & 1.529 \\
\hline 1.125 & 4.625 & 4.765 & 3.546 & 1.965 & 1.885 & 1.679 & 1.534 & 1.434 & 1.938 & 0.474 & 0.482 & 0.450 & 2.973 & 3.838 & 3.099 & 2.544 & 1.438 & 1.410 \\
\hline 1.375 & 4.664 & 4.695 & 3.469 & 1.717 & 1.693 & 1.633 & 1.330 & 1.309 & 1.880 & 0.537 & 0.493 & 0.492 & 3.323 & 4.084 & 3.146 & 2.165 & 1.380 & 1.501 \\
\hline 1.625 & 4.235 & 4.272 & 3.253 & 1.730 & 1.592 & 1.596 & 1.354 & 1.290 & 1.897 & 0.488 & 0.515 & 0.489 & 3.234 & 3.901 & 3.450 & 1.920 & 1.326 & 1.475 \\
\hline 1.875 & 4.162 & 4.208 & 3.234 & 1.500 & 1.537 & 1.565 & 1.180 & 1.247 & 1.820 & 0.487 & 0.492 & 0.451 & 3.297 & 4.031 & 3.361 & 1.710 & 1.275 & 1.383 \\
\hline 5 & 3.827 & 3.238 & 2.679 & 1.519 & 1.478 & 1.317 & 1.185 & 1.442 & 1.325 & 0.512 & 0.474 & 0.420 & 3.688 & 3.471 & 3.215 & & & \\
\hline 24 & 1.732 & 1.643 & 1.399 & 0.826 & 0.725 & 0.745 & 0.863 & 0.804 & 0.852 & 0.233 & 0.253 & 0.226 & 3.076 & 2.640 & 2.875 & & & \\
\hline 48 & 0.794 & 0.661 & 0.592 & 0.411 & 0.350 & 0.457 & 0.535 & 0.442 & 0.512 & 0.115 & 0.103 & 0.106 & 2.130 & 1.531 & 2.055 & & & \\
\hline
\end{tabular}




\section{$\left[{ }^{64} \mathrm{Cu}\right] \mathrm{Cu}-N O D A G A-c L A B 2-T A T E$}

\begin{tabular}{c|cc|cc|cc|cc|cc|cc} 
Time (h) & \multicolumn{2}{|c|}{ Heart } & \multicolumn{2}{|c|}{ Kidney } & \multicolumn{2}{|c|}{ Liver } & \multicolumn{2}{c|}{ Muscle } & \multicolumn{2}{|c}{ MPC tumor } & \multicolumn{2}{c}{ Vena Cava } \\
\hline 0.001 & 0 & 0 & 0 & 0 & 0 & 0 & 0 & 0 & 0 & 0 & 0 & 0 \\
0.004 & 4.345 & 2.877 & 0.1475 & 0.1425 & 0.064 & 0.103 & 0.023 & 0.041 & 0.021 & 0.011 & 10.547 & 26.358 \\
0.007 & 8.684 & 7.867 & 1.1875 & 1.0885 & 1.33 & 1.226 & 0.08 & 0.162 & 0.219 & 0.165 & 80.171 & 80.731 \\
0.01 & 22.394 & 12.343 & 2.5355 & 1.804 & 2.15 & 3.52 & 0.218 & 0.248 & 0.405 & 0.442 & 54.9 & 16.572 \\
0.013 & 11.122 & 14.544 & 3.205 & 2.3255 & 2.5 & 2.965 & 0.329 & 0.382 & 0.473 & 0.324 & 9.411 & 4.938 \\
0.015 & 5.492 & 10.287 & 2.585 & 2.4585 & 3.138 & 2.792 & 0.376 & 0.252 & 0.487 & 0.29 & 4.144 & 6.164 \\
0.018 & 6.674 & 10.279 & 2.778 & 3.092 & 2.386 & 2.919 & 0.365 & 0.514 & 0.607 & 0.455 & 3.983 & 0.922 \\
0.021 & 7.85 & 11.962 & 2.6945 & 2.7815 & 2.669 & 3.03 & 0.268 & 0.33 & 0.644 & 0.388 & 10.927 & 3.426 \\
0.024 & 8.271 & 12.089 & 2.7745 & 2.6665 & 3.015 & 3.145 & 0.273 & 0.52 & 0.491 & 0.338 & 5.753 & 7.267 \\
0.026 & 9.73 & 10.258 & 2.703 & 2.7945 & 2.429 & 3.038 & 0.296 & 0.305 & 0.53 & 0.434 & 4.916 & 5.633 \\
0.029 & 5.16 & 8.256 & 2.7895 & 2.514 & 2.556 & 2.981 & 0.305 & 0.317 & 0.565 & 0.507 & 2.512 & 6.352 \\
0.032 & 7.252 & 7.468 & 3.152 & 2.567 & 2.655 & 4.02 & 0.328 & 0.379 & 0.627 & 0.449 & 4.098 & 2.859 \\
0.035 & 8.162 & 11.06 & 2.512 & 2.726 & 2.378 & 3.038 & 0.264 & 0.336 & 0.562 & 0.4 & 5.71 & 3.996 \\
0.038 & 6.963 & 11.44 & 2.3995 & 2.755 & 2.186 & 2.677 & 0.357 & 0.332 & 0.448 & 0.326 & 4.793 & 4.438 \\
0.04 & 7.674 & 7.432 & 2.8185 & 2.3805 & 2.139 & 2.643 & 0.313 & 0.4 & 0.573 & 0.431 & 1.864 & 2.476 \\
0.046 & 7.423 & 7.863 & 2.6095 & 2.355 & 2.504 & 2.54 & 0.316 & 0.288 & 0.581 & 0.613 & 5.546 & 4.179 \\
0.054 & 6.511 & 8.52 & 2.5785 & 2.4575 & 2.527 & 2.773 & 0.253 & 0.246 & 0.649 & 0.655 & 2.596 & 2.692 \\
0.063 & 7.821 & 7.777 & 2.7875 & 2.539 & 2.211 & 2.779 & 0.361 & 0.269 & 0.697 & 0.674 & 4.815 & 2.822 \\
0.071 & 6.92 & 9.076 & 2.4185 & 2.4625 & 2.483 & 2.947 & 0.432 & 0.338 & 0.693 & 0.716 & 4.387 & 3.206 \\
0.079 & 6.724 & 7.127 & 2.625 & 2.537 & 2.077 & 2.384 & 0.277 & 0.327 & 0.741 & 0.692 & 1.562 & 2.513
\end{tabular}




\begin{tabular}{|c|c|c|c|c|c|c|c|c|c|c|c|c|}
\hline Time (h) & \multicolumn{2}{|c|}{ Heart } & \multicolumn{2}{|c|}{ Kidney } & \multicolumn{2}{|c|}{ Liver } & \multicolumn{2}{|c|}{ Muscle } & \multicolumn{2}{|c|}{ MPC tumor } & \multicolumn{2}{|c|}{ Vena Cava } \\
\hline 0.092 & 8.01 & 8.384 & 2.501 & 2.279 & 2.346 & 2.65 & 0.373 & 0.325 & 0.746 & 0.908 & 4.657 & 2.434 \\
\hline 0.108 & 7.427 & 7.052 & 2.4015 & 2.3615 & 2.429 & 2.289 & 0.395 & 0.306 & 0.752 & 0.853 & 3.347 & 1.977 \\
\hline 0.125 & 6.626 & 8.397 & 2.3925 & 2.4325 & 2.412 & 2.635 & 0.478 & 0.347 & 0.844 & 0.903 & 2.777 & 2.07 \\
\hline 0.142 & 8.286 & 7.242 & 2.54 & 2.532 & 2.204 & 2.708 & 0.35 & 0.387 & 0.866 & 0.972 & 2.888 & 2.478 \\
\hline 0.158 & 6.381 & 7.452 & 2.4215 & 2.3775 & 2.111 & 2.683 & 0.396 & 0.302 & 0.906 & 0.976 & 4.901 & 2.839 \\
\hline 0.208 & 6.474 & 7.134 & 2.2315 & 2.2735 & 2.146 & 2.404 & 0.398 & 0.308 & 0.963 & 1.226 & 3.053 & 2.965 \\
\hline 0.292 & 5.831 & 6.951 & 2.2335 & 2.1445 & 2.1 & 2.352 & 0.413 & 0.349 & 1.065 & 1.305 & 3.81 & 2.617 \\
\hline 0.375 & 6.362 & 6.711 & 2.233 & 2.0855 & 1.889 & 2.24 & 0.428 & 0.331 & 1.207 & 1.401 & 3.659 & 2.288 \\
\hline 0.458 & 5.955 & 6.247 & 2.156 & 1.9275 & 1.883 & 2.152 & 0.383 & 0.37 & 1.212 & 1.538 & 2.402 & 2.765 \\
\hline 0.583 & 5.428 & 5.954 & 1.9915 & 1.918 & 1.779 & 2.061 & 0.405 & 0.374 & 1.32 & 1.589 & 2.618 & 1.979 \\
\hline 0.75 & 4.889 & 5.905 & 1.912 & 1.8415 & 1.761 & 1.832 & 0.418 & 0.358 & 1.479 & 1.743 & 2.308 & 1.735 \\
\hline 0.917 & 4.94 & 5.9 & 1.8635 & 1.771 & 1.709 & 1.766 & 0.463 & 0.383 & 1.57 & 1.821 & 2.288 & 1.784 \\
\hline 1.125 & 4.46 & 5.327 & 1.806 & 1.67 & 1.563 & 1.648 & 0.439 & 0.357 & 1.689 & 2.049 & 2.265 & 1.828 \\
\hline 1.375 & 4.321 & 5.163 & 1.786 & 1.6385 & 1.521 & 1.609 & 0.47 & 0.391 & 1.793 & 2.07 & 2.222 & 1.641 \\
\hline 1.625 & 4.368 & 4.645 & 1.68 & 1.6145 & 1.457 & 1.592 & 0.45 & 0.388 & 1.859 & 2.266 & 2.006 & 1.403 \\
\hline 1.875 & 3.968 & 4.561 & 1.665 & 1.597 & 1.4 & 1.495 & 0.45 & 0.403 & 1.983 & 2.462 & 1.839 & 1.511 \\
\hline 5 & 3.318 & 3.4 & 1.6635 & 1.3935 & 1.049 & 1.292 & 0.488 & 0.431 & 2.275 & 2.82 & & \\
\hline 24 & 2.103 & 2.296 & 1.184 & 1.168 & 0.747 & 0.928 & 0.349 & 0.332 & 2.862 & 3.251 & & \\
\hline 48 & 1.285 & 1.665 & 0.83 & 0.8705 & 0.649 & 0.874 & 0.248 & 0.222 & 2.223 & 2.671 & & \\
\hline
\end{tabular}




\section{$\left[{ }^{64} \mathrm{Cu}\right] \mathrm{Cu}-N O D A G A-c L A B 3-T A T E$}

\begin{tabular}{c|cc|cc|cc|cc|cc|cc} 
Time (h) & \multicolumn{2}{|c|}{ Heart } & \multicolumn{2}{|c|}{ Kidney } & \multicolumn{2}{|c|}{ Liver } & \multicolumn{2}{|c|}{ Muscle } & \multicolumn{2}{|c}{ MPC tumor } & \multicolumn{2}{c}{ Vena Cava } \\
\hline 0.001 & 0 & 0 & 0 & 0 & 0 & 0 & 0 & 0 & 0 & 0 & 0 & 0 \\
0.004 & 1.843 & 4.27 & 0.1605 & 0.1595 & 0.096 & 0.045 & 0.014 & 0.019 & 0.029 & 0.018 & 20.893 & 13.847 \\
0.007 & 7.158 & 7.229 & 0.9745 & 1.186 & 0.96 & 1.085 & 0.167 & 0.11 & 0.186 & 0.162 & 61.957 & 100.239 \\
0.01 & 9.994 & 3.013 & 2.087 & 2.24 & 2.787 & 2.518 & 0.3 & 0.208 & 0.267 & 0.206 & 64.198 & 58.491 \\
0.013 & 13.325 & 10.324 & 2.6695 & 2.9065 & 3.509 & 3.823 & 0.253 & 0.143 & 0.385 & 0.372 & 10.328 & 17.981 \\
0.015 & 9.715 & 15.164 & 2.665 & 2.8765 & 3.708 & 2.973 & 0.355 & 0.236 & 0.432 & 0.521 & 2.747 & 5.741 \\
0.018 & 7.197 & 6.93 & 3.014 & 3.175 & 3.074 & 3.626 & 0.372 & 0.461 & 0.383 & 0.51 & 3.262 & 3.805 \\
0.021 & 7.164 & 8.711 & 2.801 & 3.115 & 3.755 & 3.113 & 0.371 & 0.507 & 0.355 & 0.487 & 3.128 & 11.155 \\
0.024 & 8.031 & 17.452 & 3.1 & 3.2185 & 3.527 & 2.558 & 0.321 & 0.376 & 0.516 & 0.366 & 3.742 & 4.526 \\
0.026 & 8.669 & 7.869 & 3.1 & 3.5265 & 3.259 & 3.156 & 0.242 & 0.231 & 0.484 & 0.481 & 2.988 & 2.842 \\
0.029 & 8.35 & 7.051 & 3.0935 & 2.927 & 3.947 & 2.942 & 0.329 & 0.33 & 0.581 & 0.484 & 5.59 & 2.458 \\
0.032 & 7.769 & 9.514 & 3.5275 & 3.073 & 3.464 & 3.461 & 0.397 & 0.345 & 0.492 & 0.494 & 11.596 & 8.866 \\
0.035 & 10.422 & 8.323 & 2.9385 & 3.411 & 3.638 & 2.747 & 0.24 & 0.537 & 0.396 & 0.441 & 4.196 & 2.303 \\
0.038 & 12.249 & 4.718 & 2.689 & 3.2365 & 3.143 & 2.77 & 0.435 & 0.404 & 0.488 & 0.51 & 1.081 & 5.859 \\
0.04 & 9.681 & 13.535 & 2.9815 & 2.774 & 3.173 & 3.4 & 0.311 & 0.307 & 0.492 & 0.541 & 6.823 & 3.434 \\
0.046 & 7.895 & 8.055 & 3.002 & 2.858 & 3.213 & 2.92 & 0.373 & 0.376 & 0.553 & 0.571 & 6.367 & 5.972 \\
0.054 & 8.086 & 9.258 & 2.9665 & 3.063 & 3.411 & 2.935 & 0.368 & 0.303 & 0.622 & 0.656 & 3.207 & 8.591 \\
0.063 & 8.312 & 8.216 & 2.889 & 2.893 & 3.039 & 2.952 & 0.445 & 0.409 & 0.568 & 0.652 & 6.297 & 1.932 \\
0.071 & 7.263 & 6.063 & 2.9055 & 3.0255 & 2.986 & 2.879 & 0.39 & 0.306 & 0.755 & 0.677 & 4.022 & 4.22 \\
0.079 & 7.129 & 5.217 & 3.0315 & 2.9555 & 3.222 & 3.073 & 0.373 & 0.362 & 0.751 & 0.776 & 3.414 & 5.301
\end{tabular}




\begin{tabular}{|c|c|c|c|c|c|c|c|c|c|c|c|c|}
\hline Time (h) & \multicolumn{2}{|c|}{ Heart } & \multicolumn{2}{|c|}{ Kidney } & \multicolumn{2}{|c|}{ Liver } & \multicolumn{2}{|c|}{ Muscle } & \multicolumn{2}{|c|}{ MPC tumor } & \multicolumn{2}{|c|}{ Vena Cava } \\
\hline 0.092 & 7.661 & 9.05 & 2.8605 & 2.8465 & 3.128 & 2.819 & 0.335 & 0.275 & 0.693 & 0.803 & 4.582 & 3.913 \\
\hline 0.108 & 7.453 & 6.523 & 2.923 & 2.764 & 2.939 & 2.747 & 0.424 & 0.322 & 0.796 & 0.839 & 4.34 & 4.224 \\
\hline 0.125 & 7.05 & 6.893 & 2.8555 & 2.891 & 2.789 & 2.544 & 0.369 & 0.353 & 0.867 & 0.877 & 4.514 & 3.775 \\
\hline 0.142 & 8.695 & 5.326 & 2.8305 & 2.8395 & 2.703 & 2.456 & 0.461 & 0.354 & 0.804 & 0.899 & 2.465 & 6.107 \\
\hline 0.158 & 8.566 & 8.494 & 2.7295 & 2.8695 & 3.152 & 2.89 & 0.386 & 0.35 & 0.991 & 1.105 & 4.265 & 4.503 \\
\hline 0.208 & 6.903 & 6.754 & 2.7435 & 2.6915 & 2.687 & 2.546 & 0.41 & 0.377 & 1.134 & 1.16 & 2.899 & 3.907 \\
\hline 0.292 & 6.659 & 7.164 & 2.614 & 2.636 & 2.558 & 2.228 & 0.458 & 0.391 & 1.43 & 1.398 & 3.127 & 3.924 \\
\hline 0.375 & 6.558 & 7.011 & 2.514 & 2.4795 & 2.441 & 2.317 & 0.452 & 0.422 & 1.716 & 1.564 & 3.035 & 3.408 \\
\hline 0.458 & 6.109 & 6.441 & 2.4535 & 2.367 & 2.349 & 2.086 & 0.456 & 0.441 & 1.924 & 1.702 & 3.076 & 3.923 \\
\hline 0.583 & 5.877 & 5.496 & 2.2975 & 2.13 & 2.238 & 1.995 & 0.479 & 0.469 & 2.184 & 1.768 & 2.078 & 2.694 \\
\hline 0.75 & 5.487 & 5.478 & 2.2275 & 2.002 & 2.015 & 1.878 & 0.467 & 0.461 & 2.532 & 1.969 & 2.166 & 2.535 \\
\hline 0.917 & 4.971 & 5.753 & 2.0805 & 1.921 & 1.994 & 1.822 & 0.475 & 0.481 & 2.944 & 2.159 & 2.245 & 2.443 \\
\hline 1.125 & 4.682 & 4.832 & 1.9355 & 1.767 & 1.825 & 1.681 & 0.475 & 0.504 & 3.196 & 2.257 & 2.052 & 2.086 \\
\hline 1.375 & 4.661 & 4.967 & 2.0955 & 1.77 & 1.829 & 1.638 & 0.5 & 0.508 & 3.433 & 2.378 & 2.089 & 1.96 \\
\hline 1.625 & 4.615 & 4.852 & 2.183 & 1.6875 & 1.796 & 1.548 & 0.518 & 0.49 & 3.479 & 2.517 & 1.755 & 1.758 \\
\hline 1.875 & 4.332 & 5.179 & 2.28 & 1.6645 & 1.767 & 1.469 & 0.522 & 0.469 & 3.689 & 2.666 & 1.661 & 1.791 \\
\hline 5 & 3.393 & 3.636 & 1.757 & 1.826 & 1.413 & 1.182 & 0.438 & 0.366 & 5.366 & 4.806 & & \\
\hline 24 & 2.064 & 2.016 & 1.0945 & 1.084 & 0.897 & 0.97 & 0.326 & 0.219 & 4.525 & 5.11 & & \\
\hline 48 & 1.364 & 1.328 & 0.729 & 0.682 & 0.708 & 0.658 & 0.183 & 0.189 & 3.003 & 3.231 & & \\
\hline
\end{tabular}




\section{$\left[{ }^{64} \mathrm{Cu}\right] \mathrm{Cu}-N O D A G A-c L A B 4-T A T E$}

\begin{tabular}{l|cc|cc|cc|cc|cc|cc} 
Time (h) & \multicolumn{2}{|c|}{ Heart } & \multicolumn{2}{|c|}{ Kidney } & \multicolumn{2}{c|}{ Liver } & \multicolumn{2}{c|}{ Muscle } & \multicolumn{2}{c}{ MPC tumor } & \multicolumn{2}{c}{ Vena Cava } \\
\hline 0.001 & 0 & 0 & 0 & 0 & 0 & 0 & 0 & 0 & 0 & 0 & 0 & 0 \\
0.004 & 1.43 & 1.809 & 0.175 & 0.2075 & 0.082 & 0.234 & 0 & 0.016 & 0.01 & 0.015 & 21.333 & 15.947 \\
0.007 & 3.783 & 4.279 & 1.201 & 1.512 & 1.002 & 1.913 & 0.126 & 0.051 & 0.126 & 0.219 & 95.283 & 98.794 \\
0.01 & 12.412 & 17.401 & 2.404 & 3.0755 & 2.858 & 2.396 & 0.215 & 0.39 & 0.464 & 0.624 & 36.956 & 32.922 \\
0.013 & 7.201 & 5.729 & 2.604 & 3.0455 & 3.882 & 3.94 & 0.24 & 0.473 & 0.523 & 0.681 & 13.82 & 8.44 \\
0.015 & 9.877 & 7.096 & 2.683 & 3.23 & 3.077 & 3.28 & 0.273 & 0.233 & 0.745 & 0.753 & 8.677 & 5.009 \\
0.018 & 8.473 & 5.943 & 2.6975 & 3.119 & 2.902 & 3.873 & 0.297 & 0.382 & 0.588 & 0.94 & 6.389 & 5.984 \\
0.021 & 7.018 & 7.448 & 2.763 & 3.0965 & 3.048 & 3.555 & 0.225 & 0.209 & 0.758 & 0.786 & 18.122 & 4.292 \\
0.024 & 7.239 & 9.517 & 2.7655 & 3.2735 & 3.159 & 3.554 & 0.364 & 0.506 & 0.695 & 0.969 & 7.398 & 7.18 \\
0.026 & 6.716 & 4.509 & 2.677 & 3.1505 & 2.241 & 2.725 & 0.375 & 0.351 & 0.976 & 1.015 & 4.301 & 1.962 \\
0.029 & 7.145 & 6.97 & 2.784 & 3.02 & 2.407 & 2.778 & 0.388 & 0.337 & 0.824 & 1.128 & 5.037 & 2.896 \\
0.032 & 5.731 & 4.998 & 2.79 & 3.32 & 3.901 & 3.039 & 0.35 & 0.277 & 0.783 & 1.108 & 5.045 & 3.225 \\
0.035 & 5.283 & 8.369 & 2.6775 & 3.146 & 3.452 & 3.289 & 0.603 & 0.277 & 0.9 & 1.383 & 5.947 & 3.62 \\
0.038 & 8.96 & 8.207 & 2.6735 & 3.013 & 2.851 & 2.598 & 0.271 & 0.266 & 1.039 & 1.013 & 3.369 & 8.155 \\
0.04 & 9.056 & 6.408 & 2.9575 & 3.4465 & 4.065 & 3.718 & 0.474 & 0.4 & 1.123 & 1.286 & 3.731 & 2.921 \\
0.046 & 6.573 & 7.046 & 2.7855 & 3.14 & 2.387 & 2.526 & 0.368 & 0.356 & 1.396 & 1.598 & 4.721 & 4.2 \\
0.054 & 7.675 & 6.768 & 2.771 & 3.0625 & 2.827 & 2.642 & 0.369 & 0.433 & 1.506 & 1.767 & 3.854 & 2.57 \\
0.063 & 7.17 & 5.828 & 3.026 & 3.341 & 2.84 & 2.16 & 0.456 & 0.403 & 1.603 & 1.866 & 6.055 & 4.766 \\
0.071 & 5.068 & 5.603 & 2.871 & 3.3645 & 2.493 & 2.733 & 0.38 & 0.291 & 1.999 & 2.283 & 1.975 & 4.088 \\
0.079 & 5.304 & 7.501 & 2.9775 & 3.3695 & 2.403 & 2.64 & 0.424 & 0.393 & 2.105 & 2.309 & 5.29 & 2.113
\end{tabular}




\begin{tabular}{|c|c|c|c|c|c|c|c|c|c|c|c|c|}
\hline Time (h) & \multicolumn{2}{|c|}{ Heart } & \multicolumn{2}{|c|}{ Kidney } & \multicolumn{2}{|c|}{ Liver } & \multicolumn{2}{|c|}{ Muscle } & \multicolumn{2}{|c|}{ MPC tumor } & \multicolumn{2}{|c|}{ Vena Cava } \\
\hline 0.092 & 5.504 & 5.425 & 3.009 & 3.437 & 2.419 & 2.339 & 0.405 & 0.441 & 2.063 & 2.541 & 4.658 & 2.331 \\
\hline 0.108 & 5.767 & 5.018 & 3.1085 & 3.34 & 2.093 & 2.312 & 0.469 & 0.416 & 2.393 & 2.869 & 3.222 & 2.72 \\
\hline 0.125 & 4.352 & 4.22 & 3.051 & 3.2915 & 2.207 & 1.907 & 0.473 & 0.364 & 2.831 & 3.036 & 4.005 & 3.293 \\
\hline 0.142 & 4.924 & 6.14 & 3.094 & 3.411 & 2.029 & 2.158 & 0.573 & 0.553 & 2.747 & 3.378 & 2.848 & 2.998 \\
\hline 0.158 & 4.026 & 5.978 & 3.004 & 3.255 & 2.037 & 1.972 & 0.432 & 0.448 & 2.773 & 3.525 & 2.71 & 1.857 \\
\hline 0.208 & 4.184 & 4.653 & 2.861 & 3.169 & 1.853 & 1.759 & 0.479 & 0.409 & 3.445 & 4.058 & 2.372 & 2.522 \\
\hline 0.292 & 4.22 & 4.286 & 2.81 & 2.951 & 1.699 & 1.676 & 0.462 & 0.435 & 3.955 & 4.856 & 2.8 & 1.921 \\
\hline 0.375 & 3.917 & 4.063 & 2.709 & 2.8615 & 1.701 & 1.565 & 0.485 & 0.374 & 4.484 & 5.721 & 2.609 & 2.021 \\
\hline 0.458 & 3.535 & 3.438 & 2.6465 & 2.773 & 1.558 & 1.382 & 0.431 & 0.415 & 5.112 & 6.163 & 1.953 & 2.128 \\
\hline 0.583 & 3.553 & 3.694 & 2.631 & 2.7295 & 1.453 & 1.345 & 0.424 & 0.382 & 5.617 & 7.078 & 2.139 & 1.759 \\
\hline 0.75 & 3.397 & 3.31 & 2.5565 & 2.631 & 1.399 & 1.265 & 0.398 & 0.382 & 6.44 & 7.958 & 1.814 & 1.684 \\
\hline 0.917 & 3.106 & 2.863 & 2.4745 & 2.5265 & 1.309 & 1.18 & 0.37 & 0.351 & 7.022 & 8.583 & 1.736 & 1.472 \\
\hline 1.125 & 2.869 & 2.698 & 2.3755 & 2.4755 & 1.234 & 1.096 & 0.363 & 0.29 & 7.494 & 9.398 & 1.543 & 1.316 \\
\hline 1.375 & 2.789 & 2.55 & 2.352 & 2.402 & 1.234 & 1.023 & 0.358 & 0.293 & 8.113 & 10.065 & 1.363 & 1.216 \\
\hline 1.625 & 2.865 & 2.569 & 2.4755 & 2.3485 & 1.132 & 0.942 & 0.344 & 0.262 & 8.436 & 10.224 & 1.233 & 1.093 \\
\hline 1.875 & 2.587 & 2.467 & 2.7365 & 2.409 & 1.064 & 0.896 & 0.306 & 0.252 & 8.921 & 10.516 & 1.137 & 0.996 \\
\hline 5 & 1.437 & 1.362 & 1.978 & 1.7975 & 0.688 & 0.644 & 0.194 & 0.155 & 9.899 & 10.564 & & \\
\hline 24 & 0.307 & 0.26 & 0.672 & 0.622 & 0.302 & 0.309 & 0.045 & 0.029 & 5.527 & 5.436 & & \\
\hline 48 & 0.077 & 0.085 & 0.307 & 0.2905 & 0.213 & 0.205 & 0.023 & 0.031 & 2.411 & 2.459 & & \\
\hline
\end{tabular}




\section{$\left[{ }^{64} \mathrm{Cu}\right] \mathrm{Cu}-(S)-10$}

\begin{tabular}{|c|c|c|c|c|c|c|c|c|c|c|c|c|}
\hline Time (h) & \multicolumn{2}{|c|}{ Heart } & \multicolumn{2}{|c|}{ Kidney } & \multicolumn{2}{|c|}{ Liver } & \multicolumn{2}{|c|}{ Muscle } & \multicolumn{2}{|c|}{ MPC tumor } & \multicolumn{2}{|c|}{ Vena Cava } \\
\hline 0.001 & 0 & 0 & 0 & 0 & 0 & 0 & 0 & 0 & 0 & 0 & 0 & 0 \\
\hline 0.004 & 2.754 & 2.346 & 0.247 & 0.2905 & 0.057 & 0.153 & 0.019 & 0.024 & 0.042 & 0.027 & 14.766 & 7.853 \\
\hline 0.007 & 8.368 & 9.089 & 1.649 & 1.6645 & 0.862 & 1.327 & 0.184 & 0.161 & 0.254 & 0.222 & 102.877 & 65.506 \\
\hline 0.01 & 11.969 & 12.844 & 2.942 & 2.742 & 2.179 & 2.472 & 0.309 & 0.288 & 0.513 & 0.598 & 22.273 & 25.857 \\
\hline 0.013 & 11.159 & 10.653 & 3.4765 & 3.068 & 2.18 & 3.321 & 0.354 & 0.284 & 0.585 & 0.619 & 5.231 & 8.841 \\
\hline 0.015 & 11.721 & 13.426 & 3.5825 & 3.3945 & 2.675 & 3.48 & 0.212 & 0.341 & 0.644 & 0.631 & 5.661 & 4.3 \\
\hline 0.018 & 8.994 & 12.145 & 3.9055 & 3.4565 & 2.846 & 3.48 & 0.321 & 0.579 & 0.688 & 0.666 & 8.43 & 9.988 \\
\hline 0.021 & 11.082 & 10.771 & 3.912 & 3.263 & 1.376 & 3.101 & 0.407 & 0.366 & 0.659 & 0.695 & 3.601 & 2.197 \\
\hline 0.024 & 12.51 & 9.208 & 3.629 & 3.1415 & 3.35 & 2.573 & 0.244 & 0.435 & 0.704 & 0.713 & 5.413 & 1.395 \\
\hline 0.026 & 11.04 & 10.599 & 4.0725 & 3.39 & 2.346 & 2.806 & 0.318 & 0.372 & 0.829 & 0.82 & 2.494 & 2.981 \\
\hline 0.029 & 10.592 & 9.473 & 3.9215 & 3.234 & 1.857 & 3.175 & 0.333 & 0.295 & 0.747 & 0.734 & 3.393 & 1.476 \\
\hline 0.032 & 9.203 & 9.198 & 3.4895 & 2.942 & 2.394 & 2.654 & 0.41 & 0.266 & 0.655 & 0.812 & 3.184 & 4.028 \\
\hline 0.035 & 7.37 & 9.868 & 3.993 & 2.9785 & 2.031 & 3.283 & 0.342 & 0.273 & 0.771 & 0.854 & 5.711 & 4.155 \\
\hline 0.038 & 8.05 & 9.414 & 3.766 & 2.954 & 2.38 & 3.017 & 0.357 & 0.543 & 0.805 & 0.922 & 2.226 & 5.45 \\
\hline 0.04 & 9.229 & 9.392 & 3.2975 & 2.785 & 2.337 & 2.796 & 0.364 & 0.45 & 0.774 & 0.747 & 2.077 & 1.917 \\
\hline 0.046 & 8.219 & 9.125 & 3.253 & 2.6055 & 2.192 & 2.708 & 0.426 & 0.391 & 0.868 & 1.038 & 3.2 & 1.941 \\
\hline 0.054 & 8.98 & 8.395 & 3.094 & 2.6255 & 2.901 & 2.668 & 0.426 & 0.428 & 0.904 & 1.085 & 3.513 & 2.083 \\
\hline 0.063 & 7.765 & 8.021 & 3.3445 & 2.6 & 2.258 & 2.62 & 0.402 & 0.384 & 0.878 & 1.083 & 2.422 & 3.481 \\
\hline 0.071 & 7.252 & 8.283 & 3.176 & 2.6455 & 2.49 & 3.189 & 0.429 & 0.396 & 1.089 & 1.054 & 1.893 & 1.785 \\
\hline 0.079 & 6.649 & 9.075 & 2.8215 & 2.4505 & 2.286 & 2.893 & 0.384 & 0.48 & 1.046 & 1.129 & 1.082 & 1.199 \\
\hline
\end{tabular}




\begin{tabular}{|c|c|c|c|c|c|c|c|c|c|c|c|c|}
\hline Time (h) & \multicolumn{2}{|c|}{ Heart } & \multicolumn{2}{|c|}{ Kidney } & \multicolumn{2}{|c|}{ Liver } & \multicolumn{2}{|c|}{ Muscle } & \multicolumn{2}{|c|}{ MPC tumor } & \multicolumn{2}{|c|}{ Vena Cava } \\
\hline 0.092 & 7.024 & 6.928 & 2.789 & 2.3885 & 2.179 & 2.921 & 0.449 & 0.452 & 1.036 & 1.195 & 1.864 & 2.772 \\
\hline 0.108 & 6.943 & 7.191 & 2.5665 & 2.299 & 2.788 & 2.478 & 0.465 & 0.481 & 1.075 & 1.184 & 1.899 & 1.616 \\
\hline 0.125 & 6.8 & 7.299 & 2.3235 & 2.2035 & 2.727 & 2.759 & 0.56 & 0.503 & 1.105 & 1.218 & 2.607 & 1.996 \\
\hline 0.142 & 6.743 & 6.856 & 2.28 & 2.33 & 2.174 & 2.453 & 0.443 & 0.52 & 1.19 & 1.177 & 3.128 & 1.727 \\
\hline 0.158 & 6.443 & 6.556 & 2.2395 & 2.0715 & 2.472 & 2.565 & 0.462 & 0.46 & 1.052 & 1.23 & 2.457 & 2.23 \\
\hline 0.208 & 6.116 & 5.985 & 2.125 & 1.8905 & 2.479 & 2.412 & 0.487 & 0.447 & 1.121 & 1.203 & 2.242 & 2.515 \\
\hline 0.292 & 5.596 & 5.436 & 1.9085 & 1.7095 & 2.268 & 2.227 & 0.455 & 0.532 & 1.073 & 1.144 & 1.965 & 1.95 \\
\hline 0.375 & 5.011 & 5.108 & 1.7695 & 1.565 & 2.13 & 1.967 & 0.497 & 0.531 & 1.078 & 1.159 & 1.711 & 1.646 \\
\hline 0.458 & 4.639 & 4.863 & 1.688 & 1.4425 & 2.091 & 1.995 & 0.493 & 0.515 & 1.016 & 1.102 & 1.625 & 1.609 \\
\hline 0.583 & 4.328 & 4.49 & 1.501 & 1.294 & 1.787 & 1.795 & 0.446 & 0.483 & 1.024 & 1.063 & 1.607 & 1.526 \\
\hline 0.75 & 3.842 & 3.808 & 1.375 & 1.1745 & 1.642 & 1.59 & 0.453 & 0.49 & 0.992 & 1.055 & 1.257 & 1.155 \\
\hline 0.917 & 3.728 & 3.681 & 1.2315 & 1.1075 & 1.49 & 1.559 & 0.443 & 0.483 & 0.937 & 1.024 & 1.262 & 1.5 \\
\hline 1.125 & 3.289 & 3.259 & 1.1465 & 0.9515 & 1.432 & 1.382 & 0.416 & 0.467 & 0.947 & 0.958 & 1.057 & 1.144 \\
\hline 1.375 & 2.989 & 2.979 & 1.0095 & 0.9075 & 1.256 & 1.236 & 0.354 & 0.428 & 0.928 & 0.965 & 1.021 & 1.114 \\
\hline 1.625 & 2.811 & 2.746 & 0.928 & 0.862 & 1.189 & 1.234 & 0.379 & 0.419 & 0.899 & 0.898 & 0.789 & 0.948 \\
\hline 1.875 & 2.55 & 2.531 & 0.8795 & 0.768 & 1.178 & 1.104 & 0.348 & 0.388 & 0.878 & 0.892 & 0.865 & 0.945 \\
\hline 5 & 1.122 & 1.089 & 0.318 & 0.403 & 0.598 & 0.537 & 0.154 & 0.163 & 0.456 & 0.577 & & \\
\hline 24 & 0.065 & 0.062 & 0.0505 & 0.063 & 0.188 & 0.202 & 0.024 & 0.02 & 0.335 & 0.358 & & \\
\hline 48 & 0.029 & 0.031 & 0.0565 & 0.0655 & 0.142 & 0.156 & 0.022 & 0.017 & 0.339 & 0.426 & & \\
\hline
\end{tabular}


Table S2: Summary of calculated $A_{U C} C_{0-48 h}$ values from plots of $S U V=f(t i m e)$ for the different ${ }^{64} \mathrm{Cu}$-labeled TATE derivatives and the isolated albumin binder

\begin{tabular}{|c|c|c|c|c|c|c|c|c|}
\hline & $\begin{array}{c}{\left[{ }^{64} \mathrm{Cu}\right] \text { Cu-DOTA- }} \\
\text { TATE }\end{array}$ & $\begin{array}{c}{\left[{ }^{64} \mathrm{Cu}\right] \mathrm{Cu}-N O D A G A-} \\
\text { TATE }\end{array}$ & {$\left[{ }^{64} \mathrm{Cu}\right] \mathrm{Cu}-5$} & $\begin{array}{c}{\left[{ }^{64} \mathrm{Cu}\right] \mathrm{Cu}-} \\
\text { NODAGA-cLAB1- } \\
\text { TATE } \\
\end{array}$ & $\begin{array}{c}{\left[{ }^{64} \mathrm{Cu}\right] \mathrm{Cu}-} \\
\text { NODAGA-cLAB2- } \\
\text { TATE }\end{array}$ & $\begin{array}{c}{\left[{ }^{64} \mathrm{Cu}\right] \mathrm{Cu}-} \\
\text { NODAGA-cLAB3- } \\
\text { TATE }\end{array}$ & $\begin{array}{c}{\left[{ }^{64} \mathrm{Cu}\right] \mathrm{Cu}-} \\
\text { NODAGA-cLAB4- } \\
\text { TATE }\end{array}$ & {$\left[{ }^{64} \mathrm{Cu}\right] \mathrm{Cu}-(S)-10$} \\
\hline Tumor & 169 & 166 & 82.6 & 134 & 123 & 207 & 289 & 21.0 \\
\hline Heart & 8.16 & 4.32 & 2.15 & 93.8 & 119 & 117 & 32.9 & 25.6 \\
\hline Kidney & 14.8 & 36.0 & 11.2 & 43.5 & 58.5 & 58.8 & 47.3 & 9.73 \\
\hline Liver & 39.0 & 11.2 & 6.15 & 44.2 & 45.8 & 48.9 & 20.6 & 17.1 \\
\hline Muscle & 2.78 & 0.94 & 0.80 & 13.2 & 16.6 & 14.2 & 4.16 & 3.86 \\
\hline Vena cava & 0.67 & 0.84 & 0.73 & 4.18 & 4.63 & 5.25 & 3.81 & 2.97 \\
\hline Tumor/heart & 20.7 & 38.4 & 38.4 & 1.43 & 1.03 & 1.77 & 8.78 & 0.82 \\
\hline Tumor/kidney & 11.4 & 4.61 & 7.38 & 3.08 & 2.10 & 3.52 & 6.11 & 2.16 \\
\hline Tumor/Liver & 4.33 & 14.8 & 13.4 & 3.03 & 2.69 & 4.23 & 14.0 & 1.23 \\
\hline Tumor/muscle & 60.8 & 177 & 103 & 10.2 & 7.41 & 14.6 & 69.5 & 5.44 \\
\hline Tumor/vena cava & 252 & 198 & 113 & 32.1 & 26.6 & 39.4 & 75.9 & 7.07 \\
\hline
\end{tabular}


Table S3: Summary of kinetic parameters for selected ${ }^{64} \mathrm{Cu}$ labeled TATE derivatives

$$
\mathrm{R}+\mathrm{L} \underset{k_{\mathrm{off}, 1}}{\stackrel{k_{\mathrm{on}, 1}}{\rightleftarrows}} \mathrm{RL} \underset{k_{\mathrm{off}, 2}}{\stackrel{k_{\mathrm{on}, 2}}{\rightleftarrows}} \mathrm{R}^{*} \mathrm{~L}
$$

\begin{tabular}{|c|c|c|c|c|}
\hline compound & $k_{\text {off, } 1}\left(\min ^{-1}\right)$ & $k_{\text {on, }, 2}\left(\min ^{-1}\right)$ & $k_{\text {off }, 2}\left(\min ^{-1}\right)$ & $t_{1 / 2}(\min )$ \\
\hline 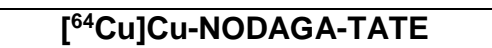 & n.d. & 0.051 & $0.012(0.017)$ & 58 \\
\hline$\left[{ }^{64} \mathrm{Cu}\right] \mathrm{Cu}-5$ & 0.150 & 0.060 & $0.005(0.002)$ & $4.6 / 139(347)$ \\
\hline$\left[{ }^{64} \mathrm{Cu}\right] \mathrm{Cu}-\mathrm{NODAGA-cLAB1-TATE}$ & 0.300 & n.d. & 0.015 & $2.3 / 46$ \\
\hline
\end{tabular}

R...receptor, L... ligand. Data shown in italic were derived from association experiments, the other data from dissociation experiments. 
Scheme S1: Synthesis of NHS ester 7a-c

Reagents and conditions: a) DIC, NHS, 1,4-dioxane, $24 \mathrm{~h}$.<smiles>[R]C(=O)ON1C(=O)CCC1=O</smiles><smiles>O=C(CCCc1ccc(I)cc1)ON1C(=O)CCC1=O</smiles>

$7 a$

$(90 \%)$<smiles>NCCCCC(=O)ON1C(=O)CCC1=O</smiles>

$7 \mathrm{~b}$

(74\%)<smiles>Nc1ccc(C(=O)ON2C(=O)CCC2=O)cc1</smiles> 


\section{Scheme S2: Synthesis of $(R)-1 p$}

Reagents and conditions: a) phenylacetylene, $\mathrm{CuSO}_{4} \times 5 \mathrm{H}_{2} \mathrm{O}$, Tris(3hydroxypropyltriazolylmethyl)amine (THPTA), sodium ascorbate, $\mathrm{H}_{2} \mathrm{O}$ /acetone $(1: 4, \mathrm{v} / \mathrm{v}), 1 \mathrm{~h}$; b) TFA/ $\mathrm{CH}_{2} \mathrm{Cl}_{2}$ (1:1, v/v), 45 min; c) IPB-NHS (7a), DIPEA, 1,4-dioxane, $2 \mathrm{~h}$.
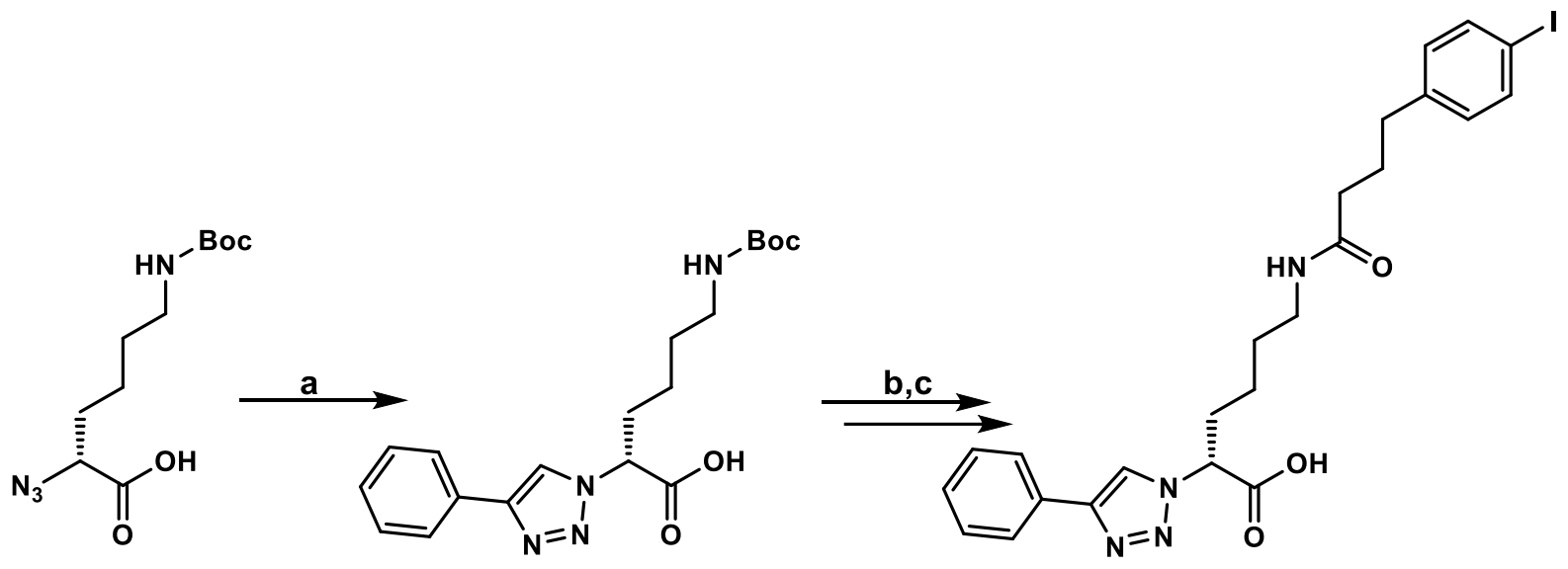

$(R)-1 \mathrm{p}$

$(65 \%)$ 


\section{Scheme S3: Synthesis of (S)-4a and (S)-4b}

Reagents and conditions: a) Rink Amide resin, HATU, DIPEA, DMF b) 20\% piperidine/DMF c) carboxylic acid, HATU, DIPEA, DMF d) TFA/H ${ }_{2} \mathrm{O} / \mathrm{TIPS}(95 / 2.5 / 2.5), 1 \mathrm{~h}$.
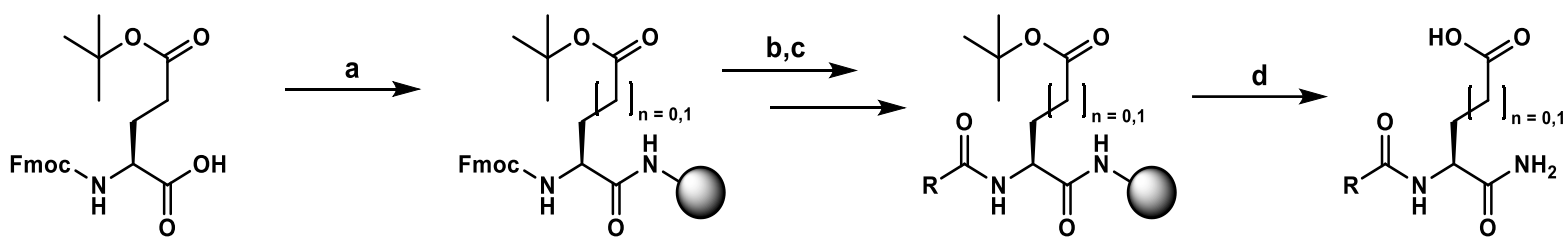

$\mathrm{n}=0 \quad(S)-4 \mathrm{a}(35 \%)$

$n=1 \quad(S)-4 b(29 \%)$ 


\section{Scheme S4:Synthesis of NODAGA-cLAB-TATEs}

Reagents and conditions: a) Fmoc/tBu-based solid-phase peptide synthesis using an automated microwave peptide synthesizer (Biotage Initiator+ Alstra) with standard protocols for coupling (HATU/DIPEA) and Fmoc removal (20\% piperidine/DMF); b) $\mathrm{CuSO}_{4} \times 5 \mathrm{H}_{2} \mathrm{O}$, THPTA, sodium ascorbate, DMF/ $\left.\mathrm{H}_{2} \mathrm{O}(6: 1, \mathrm{v} / \mathrm{v}), 2 \mathrm{~h} ; \mathrm{c}\right) \mathrm{TFA} / \mathrm{TIPS} / \mathrm{H}_{2} \mathrm{O}(95: 2.5: 2.5, \mathrm{v} / \mathrm{v} / \mathrm{v}) ; 40^{\circ} \mathrm{C}$, $2 \mathrm{~h}$; d) $10 \%$ DMSO in $\mathrm{CH}_{3} \mathrm{CN} / \mathrm{H}_{2} \mathrm{O}(25: 75, \mathrm{v} / \mathrm{v}), \mathrm{pH} 8.0,48-72 \mathrm{~h}$.

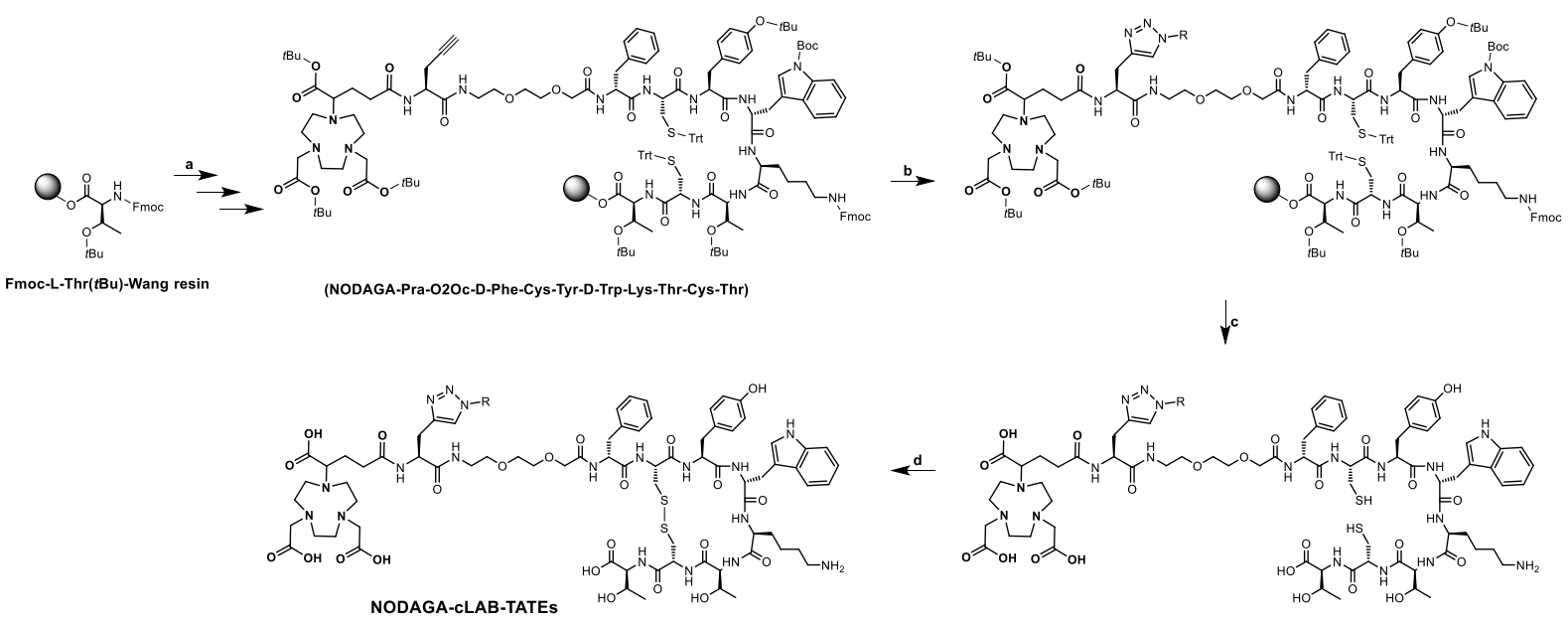




\section{Compound characterisation data}

All HPLC methods are encoded as follows:

$X \_g_{\text {start }}-g_{\text {end_ }} t_{\text {iso__t }} t_{\text {grad__f }} t_{\text {total }}$

$x \quad \mathrm{x}=\mathrm{H}$ for HPLC

$g_{\text {start }}$ concentration eluent $B$ between $t_{0}$ and start of gradient

$g_{\text {End }} \quad$ concentration of eluent $B$ end of gradient

tiso time point when gradient starts (suffix: iso)

$t_{\text {grad }}$ duration gradient in minutes (suffix: $\min$ )

$f \quad$ flow in $\mathrm{mL} / \mathrm{min}$ (suffix: $\mathrm{mL}$ )

$t_{\text {total }}$ duration of each run including wash and equilibration phase (suffix: total)

Final albumin binders

$N^{a}$-(6-FAM)- $N^{k}$-(4-lodophenylbutanoyl)-D-lysine ((R)-1a)

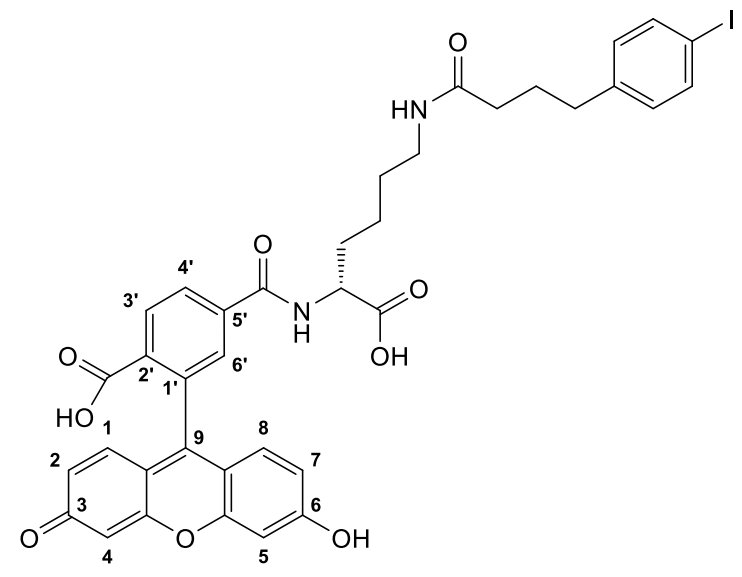

H_25-75_5iso_25min_1mL_42total $t_{R}=24.0 \mathrm{~min}$

Compound $(\boldsymbol{R})-1 \mathrm{a}$ (22 mg, 20\% overall yield, yellow solid) was synthesised starting from Fmoc-D-Lys(Alloc)-OH according to GPs I, III, V, VII, VI and VIIla but using 10 eq. 6-FAM, 10 eq. HATU and 20 eq. DIPEA (GP VI). After the coupling, an additional GP III cycle (Fmoc removal) was performed to cleave esters formed in position C3 and C6. ${ }^{15}{ }^{1} \mathrm{H}-\mathrm{NMR}$ (DMSO-d 6 , open chain numbering) $\delta=8.84\left(\mathrm{~d}, J=7.6 \mathrm{~Hz}, 1 \mathrm{H}, \mathrm{N}_{\mathrm{a}} \mathrm{H}\right), 8.22\left(\mathrm{~d}, J=8.1,1.4 \mathrm{~Hz}, 1 \mathrm{H}, \mathrm{C}{ }^{\prime} \mathrm{H}\right.$ Fluorescein), 8.09 (d, $J=7.8 \mathrm{~Hz}, 1 \mathrm{H}$, C3'H Fluorescein), 7.76 (s, 1H, C6'H Fluorescein), 7.73 (t, $J=5.7 \mathrm{~Hz}, 1 \mathrm{H}, \mathrm{N}_{\varepsilon} \mathrm{H}$ ), 7.60 (d, $J=8.2 \mathrm{~Hz}, 2 \mathrm{H}, \mathrm{H}-3,5$ lodophenyl), 6.98 (d, $J=8.2 \mathrm{~Hz}, 2 \mathrm{H}, \mathrm{H}-$ 
2,6 lodophenyl), 6.70 (t, $J=1.8 \mathrm{~Hz}, 2 \mathrm{H}, \mathrm{C}-4,5$ Fluorescein), $6.62-6.53(\mathrm{~m}, 4 \mathrm{H}, \mathrm{C}-1,2,7,8$ Fluorescein), $4.37-4.20\left(\mathrm{~m}, 1 \mathrm{H}, \mathrm{C}_{a} \mathrm{H}\right.$ Lysine), 2.97 (q, $\left.J=6.8,6.2 \mathrm{~Hz}, 2 \mathrm{H}, \mathrm{C}_{\varepsilon} \mathrm{H}_{2}\right), 2.45(\mathrm{t}, J=$ $7.5 \mathrm{~Hz}, 2 \mathrm{H}, \mathrm{C}_{\mathrm{a}} \mathrm{H}_{2}$ Butanoyl), 1.98 (t, $\mathrm{J}=7.4 \mathrm{~Hz}, 2 \mathrm{H}, \mathrm{C}_{\mathrm{\gamma}} \mathrm{H}_{2}$ Butanoyl), $1.78-1.65\left(\mathrm{~m}, 4 \mathrm{H}, \mathrm{C}_{\beta} \mathrm{H}_{2}\right.$ Butanoyl and $\mathrm{C}_{\beta} \mathrm{H}_{2}$ Lysine), $1.43-1.21$ (m, 4H, $\mathrm{C}_{\mathrm{\gamma}} \mathrm{H}_{2}$ Lysine and $\mathrm{C}_{\delta} \mathrm{H}_{2}$ Lysine). ${ }^{13} \mathrm{C}$-NMR $\left(\mathrm{DMSO}_{-} \mathrm{d}_{6}\right) \delta=173.47(\mathrm{C}=\mathrm{O}), 171.50(\mathrm{C}=\mathrm{O}), 168.01(\mathrm{C}=\mathrm{O}), 164.85(\mathrm{C}=\mathrm{O}), 159.68(\mathrm{C} 3,6$ Fluorescein), 152.54 (C9، Fluorescein), 151.85 (C-4a,5a Fluorescein), 141.57 (C1 lodophenyl), 140.02 (C5‘ Fluorescein), 137.96 (C-3,5 lodophenyl), 130.86 (C-2,6 lodophenyl), 129.74 (C4‘ Fluorescein), 129.31 (C-1,8 Fluorescein), 128.56 (C2‘ Fluorescein), 124.91 (C3، Fluorescein), 122.49 (C6، Fluorescein), 112.81 (C-2,7 Fluorescein), 109.16 (C-1a,8a Fluorescein), 102.29 (C-4,5 Fluorescein), 91.29 (C-4 lodophenyl), 52.84 ( $\mathrm{C}_{\alpha}$ Lysine), 38.08 ( $\mathrm{C}_{\varepsilon}$ Lysine), 34.61 ( $\mathrm{C}_{\alpha}$ Butanoyl), 34.01 ( $\mathrm{C}_{\mathrm{Y}}$ Butanoyl), 30.09 ( $\mathrm{C}_{\beta}$ Lysine), 28.70 ( $\mathrm{C}_{\delta}$ Lysine), 26.74 $\left(\mathrm{C}_{\beta}\right.$ Butanoyl), $23.31\left(\mathrm{C}_{\mathrm{Y}}\right.$ Lysine); MS $\left(\mathrm{ESI}^{+}\right)$: m/z calculated for $\mathrm{C}_{37} \mathrm{H}_{34} \mathrm{IN}_{2} \mathrm{O}_{9}: 777.13[\mathrm{M}+\mathrm{H}]^{+}$, found: 776.88 


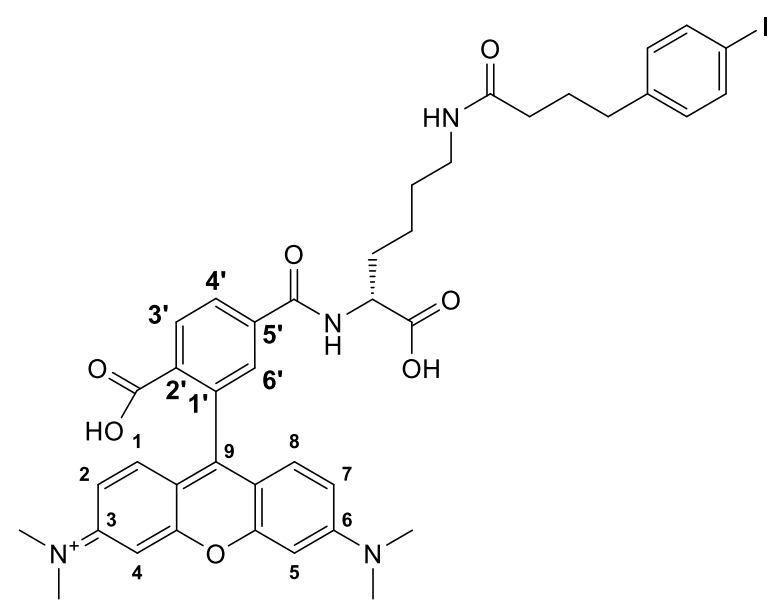

H_25-75_5iso_25min_1 $\mathrm{mL} \_42$ total $\mathrm{t}_{\mathrm{R}}=22.9 \mathrm{~min}$

Compound $(\boldsymbol{R})$-1b (45 mg, 27\% overall yield, red solid) was synthesised starting from FmocD-Lys(Alloc)-OH according to GPs I, III, V, VII, VI and VIIla. ${ }^{1} \mathbf{H}-\mathbf{N M R}\left(\mathrm{CD}_{3} \mathrm{CN}\right.$, open chain numbering) $\delta=8.30$ (d, $J=8.2 \mathrm{~Hz}, 1 \mathrm{H}, \mathrm{H} 3$ “ Rhodaminoyl), $8.17(\mathrm{dd}, J=8.2,1.8 \mathrm{~Hz}, 1 \mathrm{H}, \mathrm{H} 4$ “ Rhodaminoyl) $7.83-7.77$ (m, 2H, H6، Rhodaminoyl and $\mathrm{N}_{\alpha} \mathrm{H}$ ), 7.57 (d, J = 8.3 Hz, 2H, H-3,5 lodophenyl), 7.07 (d, $J=9.5 \mathrm{~Hz}, 1 \mathrm{H}, \mathrm{H} 1 / 8$ Rhodaminoyl), 7.02 (d, $J=9.4 \mathrm{~Hz}, 1 \mathrm{H}, \mathrm{H} 1 / 8$ Rhodaminoyl), $6.92-6.86$ (m, 4H, H-2,6 lodophenyl and H-2,7 Rhodaminoyl), 6.83 (dd, $\mathrm{J}=$ 2.5, $0.9 \mathrm{~Hz}, 2 \mathrm{H}, \mathrm{H}-4,5$ Rhodaminoyl), 6.47 (t, $J=6.1 \mathrm{~Hz}, 1 \mathrm{H}, \mathrm{N}_{\varepsilon} \mathrm{H}$ ), 4.41 (td, $J=7.8,4.8 \mathrm{~Hz}$, $1 \mathrm{H}, \mathrm{C}_{\alpha} \mathrm{H}$ lysine), $3.24\left(\mathrm{~d}, J=3.6 \mathrm{~Hz}, 12 \mathrm{H}, 4 \mathrm{xCH}_{3}\right), 3.18-3.04\left(\mathrm{~m}, 2 \mathrm{H}, \mathrm{C}_{\varepsilon} \mathrm{H}_{2}\right), 2.46-2.40(\mathrm{~m}$, $2 \mathrm{H}, \mathrm{C}_{\gamma} \mathrm{H}_{2}$ Butanoyl), 1.99 (dd, $J=7.6,6.5 \mathrm{~Hz}, 2 \mathrm{H}, \mathrm{C}_{\alpha} \mathrm{H}_{2}$ Butanoyl), $1.89-1.80\left(\mathrm{~m}, 2 \mathrm{H}, \mathrm{C}_{\beta} \mathrm{H}_{2}\right.$ Lysine), 1.69 (p, J=7.4 Hz, 2H, $\mathrm{C}_{\beta} \mathrm{H}_{2}$ Butanoyl), $1.48-1.26\left(\mathrm{~m}, 4 \mathrm{H}, \mathrm{C}_{\gamma} \mathrm{H}_{2}\right.$ and $\mathrm{C}_{\delta} \mathrm{H}_{2}$ Lysine). ${ }^{13} \mathrm{C}$ $\operatorname{NMR}\left(\mathrm{CD}_{3} \mathrm{CN}\right) \delta=173.93(\mathrm{C}=\mathrm{O}), 173.76(\mathrm{C}=\mathrm{O}), 166.40(\mathrm{C}=\mathrm{O}), 166.35(\mathrm{C}=\mathrm{O}), 158.42,158.36$, 142.84, 138.52, 138.23 (C-3,5 lodophenyl), 135.08, 134.01, 132.52 (C3، Rhodaminoyl), 131.91, 131.81, 131.76 (C-2,6 lodophenyl), 131.70, 130.01 (C4' Rhodaminoyl), 129.76 (C6'), 115.27 (C-2,7 Rhodaminoyl), 114.33, 110.98, 97.22 (C-4,5 Rhodaminoyl), 91.20 (C-4 lodophenyl), 54.07 ( $\mathrm{C}_{\alpha}$ Lysine), $41.30\left(\mathrm{CH}_{3}\right), 38.84$ ( $\mathrm{C}_{\varepsilon}$ Lysine), 36.13 ( $\mathrm{C}_{\alpha}$ Butanoyl), $35.08\left(\mathrm{C}_{\mathrm{V}}\right.$ Butanoyl), 31.04 ( $\mathrm{C}_{\beta}$ Lysine), 29.91 ( $\mathrm{C}_{\delta}$ Lysine), 27.92 ( $\mathrm{C}_{\beta}$ Butanoyl), 23.42 ( $\mathrm{C}_{\mathrm{\gamma}}$ Lysine), NMR assignment for 6-TAMRA according to data reported in literature ${ }^{16}$. MS $\left(\mathrm{ESI}^{+}\right): \mathrm{m} / \mathrm{z}$ calculated for $\mathrm{C}_{41} \mathrm{H}_{44} \mathrm{IN}_{4} \mathrm{O}_{7}: 831.22[\mathrm{M}+\mathrm{H}]^{+}$, found: 831.07 


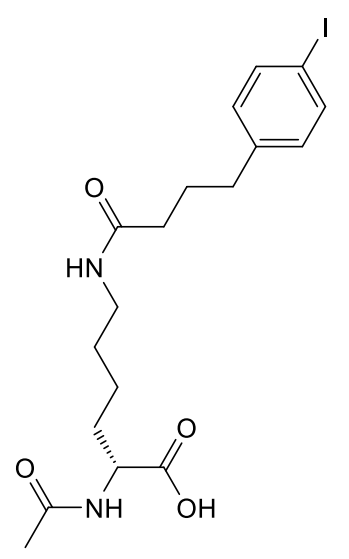

H_25-75_5iso_25min_1 $\mathrm{mL} \_42$ total $\mathrm{t}_{\mathrm{R}}=19.6 \mathrm{~min}$

Compound $(\boldsymbol{R})$-1c (34 mg, 26\% overall yield, yellow oil) was synthesised starting from FmocD-Lys(Alloc)-OH according to GPs I, III, IV, VII, VI and VIIla. ${ }^{1} \mathrm{H}-\mathrm{NMR}\left(\mathrm{CDCl}_{3}\right) \delta=7.59$ (d, $\mathrm{J}=$ $8.2 \mathrm{~Hz}, 2 \mathrm{H}, \mathrm{H}-3,5$ lodophenyl), $6.97-6.88\left(\mathrm{~m}, 3 \mathrm{H}, \mathrm{N}_{\mathrm{a}} \mathrm{H}, \mathrm{H}-2,6\right.$ lodophenyl), 5.97 (t, J=5.5 Hz, $\left.1 \mathrm{H}, \mathrm{N}_{\varepsilon} \mathrm{H}\right), 4.53\left(\mathrm{td}, J=7.6,4.7 \mathrm{~Hz}, 1 \mathrm{H}, \mathrm{C}_{\alpha} \mathrm{H}\right), 3.40-3.25\left(\mathrm{~m}, 1 \mathrm{H}, \mathrm{C}_{\varepsilon} H \mathrm{H}\right), 3.24-3.11(\mathrm{~m}, 1 \mathrm{H}$, $\mathrm{C}_{\varepsilon} \mathrm{HH}$ ), 2.58 (t, $J=7.5 \mathrm{~Hz}, 1 \mathrm{H}, \mathrm{C}_{\mathrm{\gamma}} \mathrm{H}_{2}$ Butanoyl), $2.23-2.14\left(\mathrm{~m}, 2 \mathrm{H}, \mathrm{C}_{\mathrm{a}} \mathrm{H}_{2}\right.$ Butanoyl), 2.02 (s, $3 \mathrm{H}, \mathrm{CH}_{3}$ ), $1.98-1.71$ (m, 4H, $\mathrm{C}_{\beta} \mathrm{H}_{2}$ Butanoyl, $\mathrm{C}_{\beta} \mathrm{H}_{2}$ Lysine), 1.51 (quint, $J=7.0 \mathrm{~Hz}, 2 \mathrm{H}, \mathrm{C}_{\delta} \mathrm{H}_{2}$ Lysine), $1.44-1.25$ (m, 2H, $\mathrm{C}_{\gamma} \mathrm{H}_{2}$ Lysine); ${ }^{13} \mathrm{C}-\mathrm{NMR}\left(\mathrm{CDCl}_{3}\right) \delta=174.22(\mathrm{C}=\mathrm{O}), 174.12(\mathrm{C}=\mathrm{O})$, $171.85(\mathrm{C}=\mathrm{O}), 141.01$ (C-1 lodophenyl), 137.64 (C-3,5 lodophenyl), 130.72 (C-2,6 lodophenyl), 91.28 (C-4 lodophenyl), 52.50 ( $\mathrm{C}_{\alpha}$ Lysine), 38.85 ( $\mathrm{C}_{\varepsilon}$ Lysine), 35.82 ( $\mathrm{C}_{\alpha}$ Butanoyl), 34.75 ( $\mathrm{C}_{\mathrm{Y}}$ Butanoyl), 30.97 ( $\mathrm{C}_{\beta}$ Lysine), 29.20 ( $\mathrm{C}_{\delta}$ Lysine), $27.01\left(\mathrm{C}_{\beta}\right.$ Butanoyl), $22.94\left(\mathrm{CH}_{3}\right)$, 22.05 ( $\mathrm{C}_{\mathrm{Y}}$ Lysine); $\left.\mathrm{MS}(\mathrm{ESI})^{+}\right): \mathrm{m} / \mathrm{z}$ calculated for $\mathrm{C}_{18} \mathrm{H}_{26} \mathrm{IN}_{2} \mathrm{O}_{4}: 461.09[\mathrm{M}+\mathrm{H}]^{+}$, found: 460.81

${ }^{1} \mathrm{H}-\mathrm{NMR}$ data are in agreement with those reported in literature. ${ }^{4}$ 


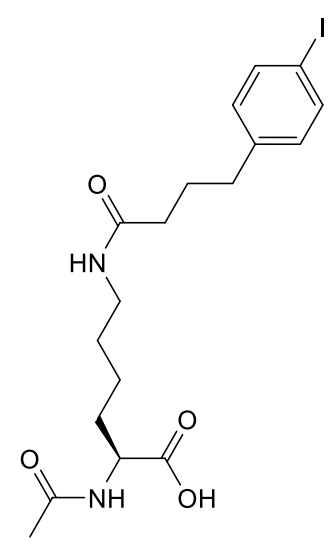

H_25-75_5iso_25min_1 $\mathrm{mL} \_42$ total $t_{R}=19.6 \mathrm{~min}$

Compound (S)-1c (22 mg, 42\% overall yield, clear colorless oil) was synthesised starting from Alloc-L-Lys(Fmoc)-OH according to GPs I, III, VI, VII, IV and VIIla. ${ }^{1} \mathbf{H}-\mathbf{N M R}\left(\mathrm{CDCl}_{3}\right) \delta=7.59$ (d, $J=8.3 \mathrm{~Hz}, 2 \mathrm{H}, \mathrm{H}-3,5$ lodophenyl), $6.98-6.87$ (m, 3H, $\mathrm{N}_{\mathrm{a}} \mathrm{H}$ and , $\mathrm{H}-2,6$ lodophenyl), 5.90 (t, $\left.J=6.0 \mathrm{~Hz}, 1 \mathrm{H}, \mathrm{N}_{\varepsilon} \mathrm{H}\right), 4.52\left(\mathrm{td}, J=7.5,4.7 \mathrm{~Hz}, 1 \mathrm{H}, \mathrm{C}_{\alpha} \mathrm{H}\right), 3.33\left(\mathrm{~m}, 1 \mathrm{H}, \mathrm{C}_{\varepsilon} H \mathrm{H}\right), 3.18(\mathrm{~m}, 5.8$ $\mathrm{Hz}, 1 \mathrm{H}, \mathrm{C}_{\varepsilon} \mathrm{HH}$ ) 2.58 (t, J = 7.5 Hz, 2H, $\mathrm{C}_{\gamma} \mathrm{H}_{2}$ Butanoyl), $2.22-2.14$ (m, 2H, $\mathrm{C}_{\alpha} \mathrm{H}_{2}$ Butanoyl), $2.02\left(\mathrm{~s}, 3 \mathrm{H}, \mathrm{CH}_{3}\right), 1.98-1.73\left(\mathrm{~m}, 4 \mathrm{H} \mathrm{C}_{\beta} \mathrm{H}_{2}\right.$ Butanoyl and $\mathrm{C}_{\beta} \mathrm{H}_{2}$ Lysine), 1.51 (quint., $J=6.9 \mathrm{~Hz}$, $2 \mathrm{H}, \mathrm{C}_{\delta} \mathrm{H}_{2}$ Lysine), $1.44-1.24$ (m, $2 \mathrm{H}, \mathrm{C}_{\mathrm{\gamma}} \mathrm{H}_{2}$ Lysine); $\mathrm{MS}(\mathrm{ESI}+)$ : $\mathrm{m} / \mathrm{z}$ calculated for $\mathrm{C}_{18} \mathrm{H}_{26} \mathrm{IN}_{2} \mathrm{O}_{4}$ : $461.09[\mathrm{M}+\mathrm{H}]^{+}$, found: 460.81

The ${ }^{13} \mathrm{C}-\mathrm{NMR}$ spectrum has not been recorded. 


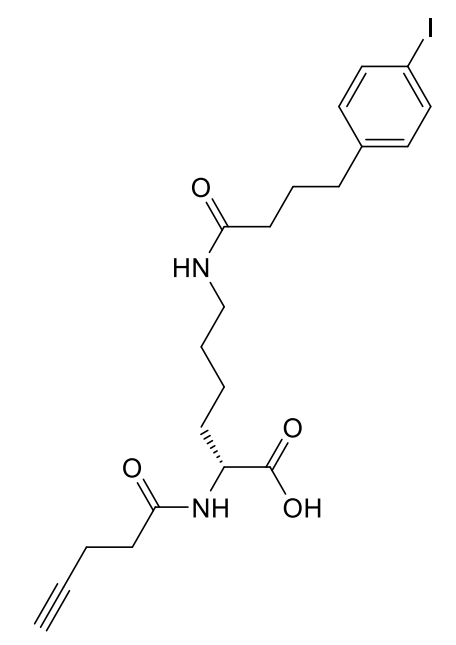

H_25-75_5iso_25min_1 $\mathrm{mL} \_42$ total $t_{R}=22.9 \mathrm{~min}$

Compound $(\boldsymbol{R}) \mathbf{- 1 d}$ (30.0 mg, 22\% overall yield, clear colorless oil) was synthesised starting from Fmoc-D-Lys(Alloc)-OH according to GPs I, III, V, VII, VI and VIIla. ${ }^{1} \mathbf{H}-\mathrm{NMR}\left(\mathrm{CDCl}_{3}\right) \delta=$ 7.59 (d, $J=8.2 \mathrm{~Hz}, 2 \mathrm{H}, \mathrm{H}-3,5$ lodophenyl), $6.95-6.86\left(\mathrm{~m}, 3 \mathrm{H}, \mathrm{N}_{\mathrm{a}} \mathrm{H}\right.$ and $\mathrm{H}-2,6$ lodophenyl), $5.93\left(\mathrm{t}, J=5.3 \mathrm{~Hz}, 1 \mathrm{H}, \mathrm{N}_{\varepsilon} \mathrm{H}\right), 4.56\left(\mathrm{td}, J=7.5,4.6 \mathrm{~Hz}, 1 \mathrm{H}, \mathrm{C}_{\alpha} \mathrm{H}\right.$ Lysine), $3.36-3.26(\mathrm{~m}, 1 \mathrm{H}$, $\mathrm{C}_{\varepsilon} \mathrm{HH}$ ), $3.23-3.14\left(\mathrm{~m}, 1 \mathrm{H}, \mathrm{C}_{\varepsilon} \mathrm{HH}\right), 2.59\left(\mathrm{t}, J=7.5 \mathrm{~Hz}, 2 \mathrm{H}, \mathrm{C}_{\mathrm{\gamma}} \mathrm{H}_{2}\right.$ Butanoyl), $2.55-2.42(\mathrm{~m}, 4 \mathrm{H}$, $\mathrm{C}_{\alpha} \mathrm{H}_{2}$ Pentynoyl and $\mathrm{C}_{\beta} \mathrm{H}_{2}$ Pentynoyl), $2.25-2.12\left(\mathrm{~m}, 2 \mathrm{H}, \mathrm{C}_{\alpha} \mathrm{H}_{2}\right.$ Butanoyl), 2.00 (t, $J=2.3 \mathrm{~Hz}$, $1 \mathrm{H}, \mathrm{C}_{\delta} \mathrm{H}$ Pentynoyl), $1.97-1.85\left(\mathrm{~m}, \mathrm{C}_{\beta} \mathrm{H}_{2}\right.$ Butanoyl and $\mathrm{C}_{\beta} H \mathrm{H}$ Lysine $1.86-1.72\left(\mathrm{~m}, 1 \mathrm{H}, \mathrm{C}_{\beta} \mathrm{HH}\right.$ Lysine), 1.52 ( $\mathrm{p}, \mathrm{J}=7.1 \mathrm{~Hz}, 2 \mathrm{H}, \mathrm{C}_{\delta} \mathrm{H}_{2}$ Lysine), $1.45-1.24$ (m, 2H, $\mathrm{C}_{\mathrm{\gamma}} \mathrm{H}_{2}$ Lysine). ${ }^{13} \mathrm{C}-\mathrm{NMR}$ $\left(\mathrm{CDCl}_{3}\right) \delta=174.20(2 \times \mathrm{C}=\mathrm{O}), 172.17$ (C=O Butanoyl), 141.04 (C1 lodophenyl), 137.63 (C-3,5 lodophenyl), 130.75 (C2,6-lodophenyl), 91.27 (C4 lodophenyl), 82.84 ( $\mathrm{C}_{\mathrm{Y}}$ Pentynoyl), 69.72

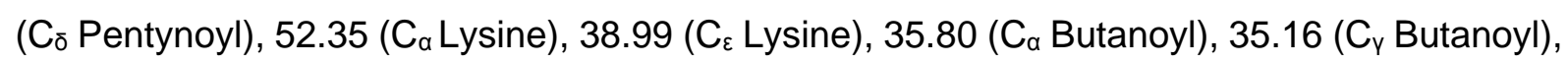
34.77 ( $\mathrm{C}_{\alpha}$ Pentynoyl), 31.20 ( $\mathrm{C}_{\beta}$ Butanoyl), 29.12 ( $\mathrm{C}_{\delta}$ Lysine), 27.02 ( $\mathrm{C}_{\beta}$ Lysine), $22.11\left(\mathrm{C}_{\mathrm{V}}\right.$ Lysine), $14.99\left(\mathrm{C}_{\beta}\right.$ Pentynoyl); $\mathrm{MS}\left(\mathrm{ESI}^{+}\right): \mathrm{m} / \mathrm{z}$ calculated for $\mathrm{C}_{21} \mathrm{H}_{28} \mathrm{IN}_{2} \mathrm{O}_{4}: 499.11[\mathrm{M}+\mathrm{H}]^{+}$, found: 498.94 


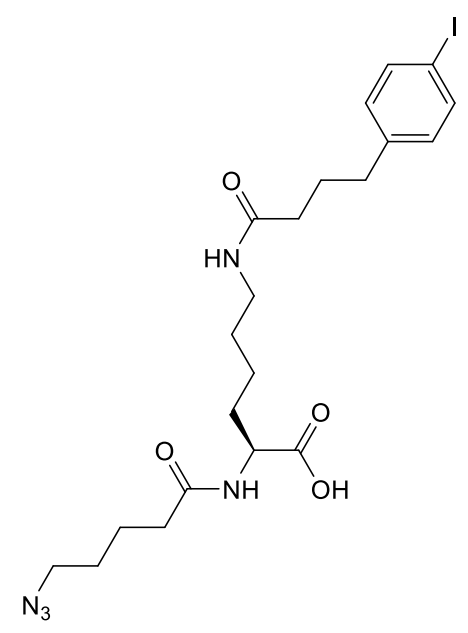

H_25-75_5iso_25min_1 $\mathrm{mL} \_42$ total $\mathrm{t}_{\mathrm{R}}=22.3 \mathrm{~min}$

Compound (S)-1e (62.0 mg, 25\% overall yield, clear yellow oil) was synthesised starting from Alloc-D-Lys(Fmoc)-OH according to GPs I, III, IV, VII, VI and VIIla. ${ }^{1} \mathrm{H}-\mathrm{NMR}\left(\mathrm{CDCl}_{3}\right) \delta=7.60$ (d, $J=8.3 \mathrm{~Hz}, 2 \mathrm{H}, \mathrm{H}-3,5$ lodophenyl), $6.92(\mathrm{~d}, J=8.2 \mathrm{~Hz}, 2 \mathrm{H} \mathrm{H}-2,6$ lodophenyl), 6.83 (d, $J=$ $\left.7.3 \mathrm{~Hz}, 1 \mathrm{H}, \mathrm{N}_{\mathrm{a}} \mathrm{H}\right), 5.83\left(\mathrm{t}, J=6.1 \mathrm{~Hz}, 1 \mathrm{H}, \mathrm{N}_{\varepsilon} \mathrm{H}\right), 4.53(\mathrm{td}, J=7.4,4.7 \mathrm{~Hz}, 1 \mathrm{H}), 3.39-3.30(\mathrm{~m}$, $1 \mathrm{H}, \mathrm{C}_{\varepsilon} H \mathrm{H}$ ), $3.27\left(\mathrm{t}, J=6.7 \mathrm{~Hz}, 2 \mathrm{H}, \mathrm{C}_{\delta} \mathrm{H}_{2}\right.$ Azidopentanoyl), $3.23-3.13\left(\mathrm{~m}, 1 \mathrm{H}, \mathrm{C}_{\varepsilon} \mathrm{HH}\right), 2.59$ (t, $J=7.5 \mathrm{~Hz}, 2 \mathrm{H}, \mathrm{C}_{\mathrm{\gamma}} \mathrm{H}_{2}$ Butanoyl), 2.28 (td, $J=7.2,3.5 \mathrm{~Hz}, 2 \mathrm{H} \mathrm{C}_{\mathrm{a}} \mathrm{H}_{2}$ Azidopentanoyl), 2.19 (t, $J=$ $7.5 \mathrm{~Hz}, 2 \mathrm{H}, \mathrm{C}_{\alpha} \mathrm{H}_{2}$ Butanoyl), $1.98-1.77$ (m, 4H, $\mathrm{C}_{\beta} \mathrm{H}_{2}$ Butanoyl and $\mathrm{C}_{\beta} \mathrm{H}_{2}$ Lysine), $1.75-1.66$ (m, 2H, $\mathrm{C}_{\beta} \mathrm{H}_{2}$ Azidopentanoyl), $1.64-1.56$ (m, 2H, $\mathrm{C}_{\gamma} \mathrm{H}_{2}$ Azidopentanoyl), 1.52 (p, J=7.1 Hz, $2 \mathrm{H}, \mathrm{C}_{\delta} \mathrm{H}_{2}$ Lysine $), 1.44-1.24\left(\mathrm{~m}, 2 \mathrm{H}, \mathrm{C}_{\mathrm{\gamma}} \mathrm{H}_{2}\right.$ Lysine). ${ }^{13} \mathrm{C}-\mathrm{NMR}\left(\mathrm{CDCl}_{3}\right) \delta=174.3(\mathrm{C}=\mathrm{O}), 174.05$ $(\mathrm{C}=\mathrm{O}), 173.98(\mathrm{C}=\mathrm{O}), 140.99$ (C-1 lodophenyl), 137.65 (C-3,5 lodophenyl), 130.72 (C-2,6 lodophenyl), 91.30 (C-4 lodophenyl), 52.41 ( $\mathrm{C}_{\alpha}$ Lysine), 51.23 ( $\mathrm{C}_{\alpha}$ Lysine), 38.86 ( $\mathrm{C}_{\varepsilon}$ Lysine), 35.76 ( $\mathrm{C}_{\alpha}$ Butanoyl), 35.59 ( $\mathrm{C}_{\alpha}$ Azidopentanoyl), 34.75 ( $\mathrm{C}_{\mathrm{y}}$ Butanoyl), 30.86 ( $\mathrm{C}_{\beta}$ Butanoyl), 29.21 ( $C_{\delta}$ Lysine), 28.41 ( $C_{\beta}$ Azidopentanoyl), 27.04 ( $C_{\beta}$ Lysine), 22.88 ( $C_{\mathrm{Y}}$ Azidopentanoyl), $22.07\left(\mathrm{C}_{\mathrm{Y}}\right.$ Lysine); $\left.\mathrm{MS}(\mathrm{ESI})^{+}\right): \mathrm{m} / \mathrm{z}$ calculated for $\mathrm{C}_{21} \mathrm{H}_{31} \mathrm{IN}_{5} \mathrm{O}_{4}: 544.14[\mathrm{M}+\mathrm{H}]^{+}$, found: 543.78 


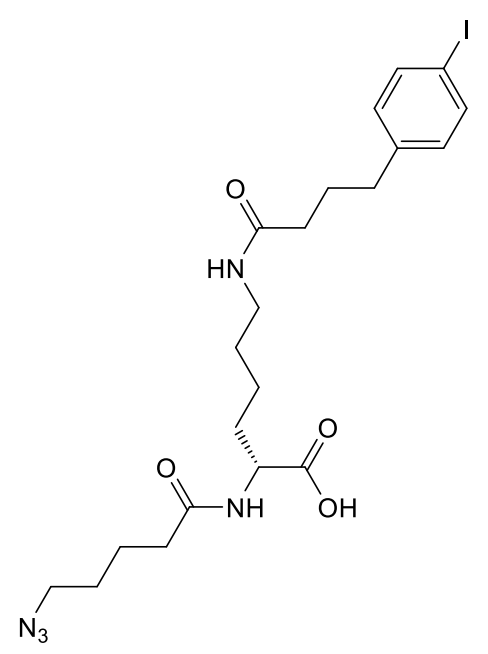

H_25-75_5iso_25min_1 $1 \mathrm{~mL} \_42$ total $t_{R}=22.3 \mathrm{~min}$

Compound $(\boldsymbol{R})-\mathbf{1 e}$ (50 mg, 23\% over two steps, clear colorless oil) was synthesised starting from Fmoc-D-Lys(Alloc)-OH according to GPs $\mathbf{X}$ and XI. ${ }^{1} \mathbf{H}-\mathrm{NMR}\left(\mathrm{CDCl}_{3}\right) \delta=7.60$ (d, $J=8.3$ $\mathrm{Hz}, 2 \mathrm{H}, \mathrm{H}-3,5$ lodophenyl), $6.92(\mathrm{~d}, J=8.2 \mathrm{~Hz}, 2 \mathrm{H}, \mathrm{H}-2,6$ lodophenyl), 6.85 (d, J = 7.1 Hz, $1 \mathrm{H}$, $\left.\mathrm{N}_{\mathrm{a}} \mathrm{H}\right), 5.91\left(\mathrm{~s}, 1 \mathrm{H}, \mathrm{N}_{\varepsilon} \mathrm{H}\right), 4.52\left(\mathrm{~d}, J=6.1 \mathrm{~Hz}, 1 \mathrm{H}, \mathrm{C}_{\alpha} \mathrm{H}\right.$ Lysine), $3.38-3.30\left(\mathrm{~m}, 1 \mathrm{H}, \mathrm{C}_{\varepsilon} H \mathrm{H}\right), 3.27$ (t, J = 6.7 Hz, 2H, $\mathrm{C}_{\delta} \mathrm{H}_{2}$ Azidopentanoyl), $3.24-3.14\left(\mathrm{~m}, 1 \mathrm{H}, \mathrm{C}_{\varepsilon} \mathrm{HH}\right.$ ), 2.59 (t, J=7.5 Hz, 2H, $\mathrm{C}_{\mathrm{\gamma}} \mathrm{H}_{2}$ Butanoyl), 2.28 (td, $J=7.2,3.5 \mathrm{~Hz}, 2 \mathrm{H} \mathrm{C}_{a} \mathrm{H}_{2}$ Azidopentanoyl), 2.19 (t, $J=7.5 \mathrm{~Hz}, 2 \mathrm{H}$, $\mathrm{C}_{\alpha} \mathrm{H}_{2}$ Butanoyl), 1.93 (p, J=7.8 Hz, 2H, $\mathrm{C}_{\beta} \mathrm{H}_{2}$ Butanoyl), 1.71 (p, J=6.9 Hz, 2H, $\mathrm{C}_{\beta} \mathrm{H}_{2}$ Lysine), 1.60 (q, $J=7.0 \mathrm{~Hz}, 2 \mathrm{H}, \mathrm{C}_{\beta} \mathrm{H}_{2}$ Azidopentanoyl $1.58-1.46$ (m, 2H, $\mathrm{C}_{\gamma} \mathrm{H}_{2}$ Azidopentanoyl), 1.45 - $1.22\left(\mathrm{~m}, 4 \mathrm{H}, \mathrm{C}_{\delta} \mathrm{H}_{2}\right.$ Lysine and $\mathrm{C}_{\gamma} \mathrm{H}_{2}$ Lysine). ${ }^{13} \mathrm{C}-\mathrm{NMR}\left(\mathrm{CDCl}_{3}\right) \delta=174.13(\mathrm{C}=\mathrm{O}), 174.04$ $(\mathrm{C}=\mathrm{O}), 173.92(\mathrm{C}=\mathrm{O}), 141.02$ (C-1 lodophenyl), 137.65 (C-3,5 lodophenyl), 130.73 (C-2,6 lodophenyl), 91.29 (C-4 lodophenyl), 52.43 ( $\mathrm{C}_{\alpha}$ Lysine), 51.2 ( $\mathrm{C}_{\alpha}$ Lysine), 38.82 ( $\mathrm{C}_{\varepsilon}$ Lysine), 35.79 ( $\mathrm{C}_{\alpha}$ Butanoyl), 35.60 ( $\mathrm{C}_{\alpha}$ Azidopentanoyl), 34.76 ( $\mathrm{C}_{\mathrm{y}}$ Butanoyl), $30.84\left(\mathrm{C}_{\beta}\right.$ Butanoyl), 29.25 ( $C_{\delta}$ Lysine), 28.43 ( $C_{\beta}$ Azidopentanoyl), 27.05 ( $C_{\beta}$ Lysine), 22.87 ( $C_{\gamma}$ Azidopentanoyl), $22.08\left(\mathrm{C}_{\mathrm{Y}}\right.$ Lysine); $M S\left(E S I^{+}\right): \mathrm{m} / \mathrm{z}$ calculated for $\mathrm{C}_{21} \mathrm{H}_{31} \mathrm{IN}_{5} \mathrm{O}_{4}: 544.14[\mathrm{M}+\mathrm{H}]^{+}$, found: 543.98 


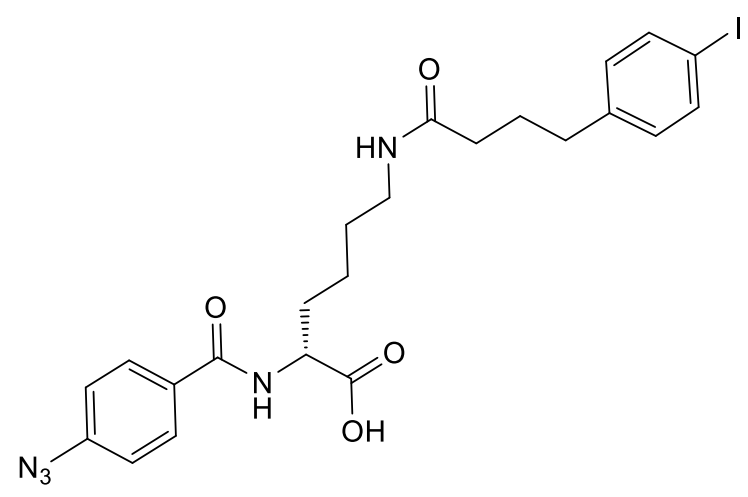

H_25-75_5iso_25min_1 $\mathrm{mL} \_42$ total $\mathrm{t}_{\mathrm{R}}=24.7 \mathrm{~min}$

Compound $(\boldsymbol{R})-\mathbf{1 f}$ (10 $\mathrm{mg}, 6 \%$ over both steps, slightly yellow solid) was synthesised starting from D-Lysine* $\mathrm{HCl}$ according to GPs $\mathbf{X}$ and $\mathbf{X I}$ (4-azidobenzoic acid activated as NHS ester). ${ }^{1} \mathrm{H}-\mathrm{NMR}\left(\mathrm{DMSO}_{\mathrm{d}}\right) \delta 8.56\left(\mathrm{~d}, J=7.6 \mathrm{~Hz}, 1 \mathrm{H}, \mathrm{N}_{\mathrm{a}} \mathrm{H}\right), 7.94(\mathrm{~d}, J=8.6 \mathrm{~Hz}, 2 \mathrm{H}, \mathrm{H}-2,6$ Azidobenzoyl), 7.76 (t, $J=5.6 \mathrm{~Hz}, 1 \mathrm{H}, \mathrm{N}_{\varepsilon} \mathrm{H}$ ), 7.60 (d, $J=8.2 \mathrm{~Hz}, 2 \mathrm{H}, \mathrm{H}-3,5$ lodophenyl), 7.19 (d, J = 8.6 Hz, 2H, H-3,5 Azidobenzoyl), 6.98 (d, J = 8.2 Hz, 2H, H-2,6 lodophenyl), 4.33 (td, J $=8.1,7.6,5.2 \mathrm{~Hz}, 1 \mathrm{H}, \mathrm{C}_{\mathrm{a}} \mathrm{H}$ Lysine), 3.01 (q, $\left.J=6.1 \mathrm{~Hz}, 2 \mathrm{H} \mathrm{C}_{\varepsilon} \mathrm{H}_{2}\right), 2.50-2.43^{*}\left(\mathrm{~m}, 2 \mathrm{H}, \mathrm{C}_{\gamma} \mathrm{H}_{2}\right.$ Butanoyl), 2.02 (t, $J=7.4 \mathrm{~Hz}, 2 \mathrm{H}, \mathrm{C}_{\alpha} \mathrm{H}_{2}$ Butanoyl), $1.84-1.64\left(\mathrm{~m}, 4 \mathrm{H}, \mathrm{C}_{\beta} \mathrm{H}_{2}\right.$ Lysine and $\mathrm{C}_{\beta} \mathrm{H}_{2}$ Butanoyl), $1.45-1.28\left(\mathrm{~m}, 4 \mathrm{H}, \mathrm{C}_{\delta} \mathrm{H}_{2} \mathrm{C}_{\gamma} \mathrm{H}_{2}\right.$ Lysine). ${ }^{*}$ Signal of overlaps with solvent peak of DMSO. Determined by HSQC but no clear assignment possible. ${ }^{13} \mathrm{C}-\mathrm{NMR}$ (DMSO- $\mathrm{d}_{6}$ ) $\delta 173.77$ $(\mathrm{C}=\mathrm{O}), 171.52(\mathrm{C}=\mathrm{O}), 165.55(\mathrm{C}=\mathrm{O}), 142.40$ (C-4 Azidobenzoyl), 141.55 (C-4 lodophenyl), 136.93 (C-3,5 lodophenyl), 130.83 (C-2,6 lodophenyl), 130.48 (C-1 Azidobenzoyl), 129.37 (C2,6 Azidobenzoyl), 118.81 (C-3,5 Azidobenzoyl), 91.23 (C-1 lodophenyl), 52.59 (C a $_{\alpha}$ Lysine), 38.10 ( $C_{\varepsilon}$ Lysine), 34.65 ( $C_{\alpha}$ Butanoyl), 34.01 ( $C_{\gamma}$ Butanoyl), $30.19\left(\mathrm{C}_{\beta}\right.$ Lysine), $28.77\left(\mathrm{C}_{\delta}\right.$ Lysine), 26.74 ( $\mathrm{C}_{\beta}$ Butanoyl), 23.29 ( $\mathrm{C}_{\mathrm{Y}}$ Lysine); MS (ESI+): m/z calculated for $\mathrm{C}_{23} \mathrm{H}_{27} \mathrm{IN}_{5} \mathrm{O}_{4}$ : $564.11[\mathrm{M}+\mathrm{H}]^{+}$, found: 563.80 


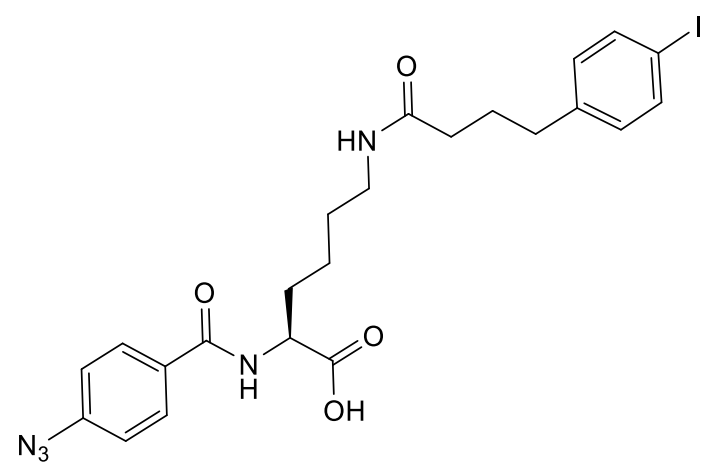

H_25-75_5iso_25min_1 $\mathrm{mL} \_42$ total $\mathrm{t}_{\mathrm{R}}=24.7 \mathrm{~min}$

Compound (S)-1f (52 mg, 34\% overall yield, slightly yellow solid) was synthesised starting from Boc-L-Lysine-OH according to GPs XI, XIV and XII. ${ }^{1} \mathrm{H}-\mathrm{NMR}\left(\mathrm{DMSO}^{\left.-\mathrm{d}_{6}\right)} \delta=8.52(\mathrm{~d}, J=7.6\right.$ $\mathrm{Hz}, 1 \mathrm{H}, \mathrm{N}_{\mathrm{a}} \mathrm{H}$ ), 7.90 (d, J=8.6 Hz, 2H, H-2,6 Azidobenzoyl), $7.73\left(\mathrm{t}, J=5.7 \mathrm{~Hz}, 1 \mathrm{H}, \mathrm{N}_{\varepsilon} \mathrm{H}\right.$ ), 7.57 (d, J = 8.2 Hz, 2H, H-3,5 lodophenyl), 7.16 (d, J = 8.6 Hz, 2H, H-3,5 Azidobenzoyl), 6.95 (d, J $=8.3 \mathrm{~Hz}, 2 \mathrm{H}, \mathrm{H}-2,6$ lodophenyl), $4.33-4.25$ (m, $1 \mathrm{H}, \mathrm{C}_{\alpha} \mathrm{H}$ Lysine), 2.99 (q, $\mathrm{J}=6.3 \mathrm{~Hz}, 2 \mathrm{H}, \mathrm{C}_{\varepsilon} \mathrm{H}_{2}$ Lysine), 2.48 - 2.39 (m, 2H, $\mathrm{C}_{\mathrm{a}} \mathrm{H}_{2}$ Butanoyl), 1.99 (t, $J=7.4 \mathrm{~Hz}, 2 \mathrm{H}, \mathrm{C}_{\mathrm{\gamma}} \mathrm{H}_{2}$ Butanoyl), 1.81 $1.64\left(\mathrm{~m}, 4 \mathrm{H}, \mathrm{C}_{\beta} \mathrm{H}_{2}\right.$ Lysine and $\mathrm{C}_{\beta} \mathrm{H}_{2}$ Butanoyl), $1.41-1.26\left(\mathrm{~m}, 4 \mathrm{H}, \mathrm{C}_{\mathrm{\gamma}} \mathrm{H}_{2}\right.$ Lysine and $\mathrm{C}_{\delta} \mathrm{H}_{2}$ Lysine). ${ }^{13} \mathrm{C}-\mathrm{NMR}$ (DMSO-d $\left.\mathrm{d}_{6}\right) \delta=173.81(\mathrm{C}=\mathrm{O}), 171.60(\mathrm{C}=\mathrm{O}), 165.61(\mathrm{C}=\mathrm{O}), 142.44(\mathrm{C}-4$ Azidobenzoyl), 141.58 (C-4 lodophenyl), 136.96 (C-3,5 lodophenyl), 130.86 (C-2,6 lodophenyl), 130.50 (C-1 Azidobenzoyl), 129.40 (C-2,6 Azidobenzoyl), 118.84 (C-3,5 Azidobenzoyl), 91.25 (C-1 lodophenyl), 52.63 ( $\mathrm{C}_{\alpha}$ Lysine), 38.14 ( $\mathrm{C}_{\varepsilon}$ Lysine), 34.68 ( $\mathrm{C}_{\alpha}$ Butanoyl), 34.04 ( $\mathrm{C}_{\mathrm{\gamma}}$ Butanoyl), 30.21 ( $\mathrm{C}_{\beta}$ Lysine), 28.78 ( $\mathrm{C}_{\delta}$ Lysine), $26.78\left(\mathrm{C}_{\beta}\right.$ Butanoyl), $23.31\left(\mathrm{C}_{\mathrm{Y}}\right.$ Lysine); $\mathrm{MS}\left(\mathrm{ESI}^{+}\right)$: $\mathrm{m} / \mathrm{z}$ calculated for $\mathrm{C}_{23} \mathrm{H}_{27} \mathrm{IN}_{5} \mathrm{O}_{4}: 564.11[\mathrm{M}+\mathrm{H}]^{+}$, found: 563.70 


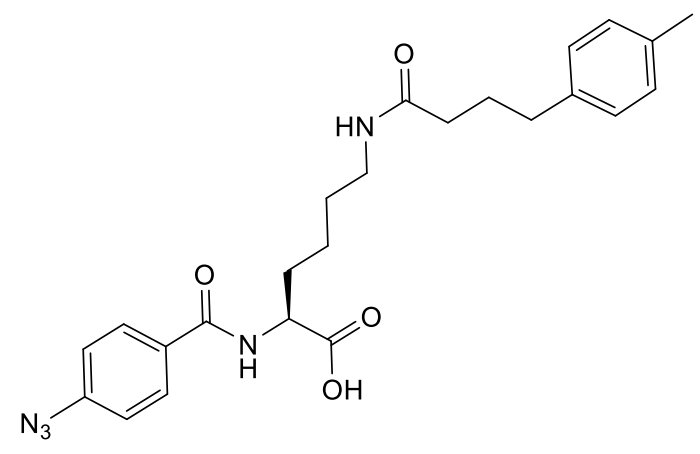

H_25-75_5iso_25min_1 $\mathrm{mL} \_42$ total $\mathrm{t}_{\mathrm{R}}=26.8 \mathrm{~min}$

Compound (S)-1g (38 mg, 41\% overall yield, slightly yellow solid) was synthesised starting from Boc-L-Lysine-OH according to GPs XI, XIV and XII. ${ }^{1} \mathrm{H}-\mathrm{NMR}\left(\mathrm{CDCl}_{3}\right) \delta=7.79(\mathrm{~d}, J=8.6$ $\mathrm{Hz}, 2 \mathrm{H}, \mathrm{H}-2,6$ Azidobenzoyl), 7.62 (d, J = 7.9 Hz, 1H, Na $\mathrm{H}$ ), 7.06 (d, J=7.6 Hz, 2H, 2H Tolyl), $6.98(\mathrm{~d}, J=8.0 \mathrm{~Hz}, 2 \mathrm{H}, 2 \mathrm{H}$ Tolyl), 6.87 (d, $J=8.6 \mathrm{~Hz}, 2 \mathrm{H}, \mathrm{H}-3,5$ Azidobenzoyl), 5.86 (t, $J=6.1$ $\mathrm{Hz}, 1 \mathrm{H}, \mathrm{N}_{\varepsilon} \mathrm{H}$ ), $4.86\left(\mathrm{q}, J=7.3 \mathrm{~Hz}, 1 \mathrm{H}, \mathrm{C}_{\mathrm{a}} \mathrm{H}\right.$ Lysine), $3.53-3.39\left(\mathrm{~m}, 1 \mathrm{H}, 1 \mathrm{xH} \mathrm{C}_{\varepsilon} \mathrm{H}_{2}\right.$ Lysine), 3.21 - 3.09 (m, 1H, 1xH C $\mathrm{H}_{2}$ Lysine), 2.53 (t, $J=7.4 \mathrm{~Hz}, 2 \mathrm{H}, \mathrm{C}_{\mathrm{\gamma}} \mathrm{H}_{2}$ Butanoyl), 2.30 (s, 3H, $\mathrm{CH}_{3}$ Tolyl), 2.22 - 2.12 ( $\mathrm{m}, 2 \mathrm{H}, \mathrm{C}_{\alpha} \mathrm{H}_{2}$ Butanoyl) $2.03-1.88$ (m, 2H, $\mathrm{C}_{\beta} \mathrm{H}_{2}$ Lysine), $1.93-1.80$ (m, $2 \mathrm{H}, \mathrm{C}_{\beta} \mathrm{H}_{2}$ Butanoyl), $1.61-1.32\left(\mathrm{~m}, \mathrm{C}_{\delta} \mathrm{H}_{2}\right.$ Lysine and $\mathrm{C}_{\gamma} \mathrm{H}_{2}$ Lysine). ${ }^{13} \mathrm{C}-\mathrm{NMR}\left(\mathrm{CDCl}_{3}\right) \delta=$ $175.19(\mathrm{C}=\mathrm{O}), 174.91(\mathrm{C}=\mathrm{O}), 167.02(\mathrm{C}=\mathrm{O}), 143.80$ (C-4 Azidobenzoyl), 137.98 (C-1/4 Tolyl), 135.74 (C-1/4 Tolyl), 129.60 (C-1 Azidobenzoyl), 129.29 (2xCH Tolyl), 129.19 (C-2,6 Azidobenzoyl), 128.39 (2xCH Tolyl), 118.98 (C-3,5 Azidobenzoyl), 52.68 (C $\alpha$ Lysine), 38.77 ( $C_{\varepsilon}$ Lysine), 35.95 ( $\mathrm{C}_{\alpha}$ Butanoyl), 34.71 ( $\mathrm{C}_{\mathrm{Y}}$ Butanoyl), 31.21 ( $\mathrm{C}_{\beta}$ Lysine), 29.14 ( $\mathrm{C}_{\mathrm{y}}$ Lysine), $27.26\left(\mathrm{C}_{\beta}\right.$ Butanoyl), $22.16\left(\mathrm{C}_{\mathrm{\gamma}}\right.$ Lysine), $21.12\left(\mathrm{CH}_{3}\right.$ Tolyl); MS (ESI+): m/z calculated for $\mathrm{C}_{24} \mathrm{H}_{30} \mathrm{~N}_{5} \mathrm{O}_{4}: 452.23[\mathrm{M}+\mathrm{H}]^{+}$, found: 451.89 


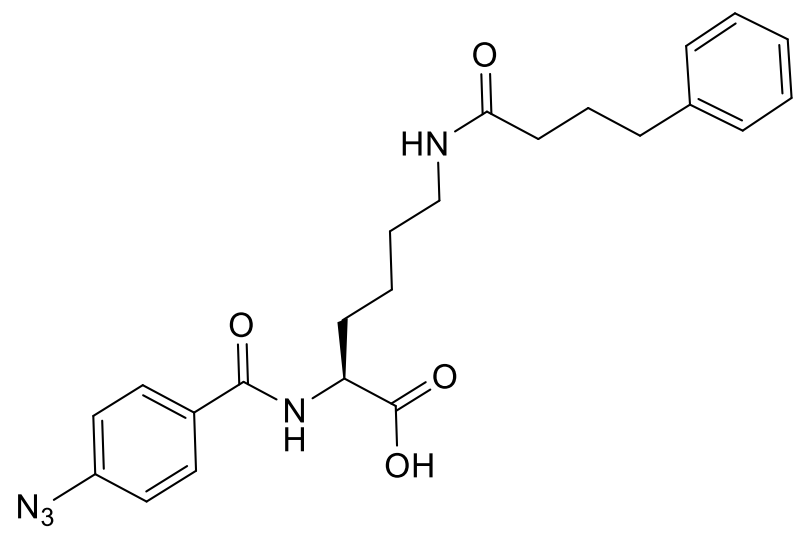

H_25-75_5iso_25min_1 $1 \mathrm{~mL} \_42$ total $t_{R}=25.0 \mathrm{~min}$

Compound (S)-1h (10 mg, 38\% overall yield, white solid) was synthesised starting from BocL-Lysine-OH according to GPs XI, XIV and XII. ${ }^{1} \mathrm{H}-\mathrm{NMR}$ (DMSO-d 6 ) $\delta=12.55$ (br s, $1 \mathrm{H}$, $\mathrm{COOH}), 8.56\left(\mathrm{~d}, J=7.6 \mathrm{~Hz}, 1 \mathrm{H}, \mathrm{N}_{\mathrm{a}} \mathrm{H}\right.$ ), 7.94 (d, $J=8.6 \mathrm{~Hz}, 2 \mathrm{H}, \mathrm{H}-2,6$ Azidobenzoyl), 7.76 (t, $J$ $=5.7 \mathrm{~Hz}, 1 \mathrm{H}, \mathrm{N}_{\varepsilon} \mathrm{H}$ ), $7.26(\mathrm{t}, J=7.6 \mathrm{~Hz}, 2 \mathrm{H}, 2 \times \mathrm{CH}$ - Phenyl), 7.19 (d, $J=8.6 \mathrm{~Hz}, 2 \mathrm{H}, \mathrm{H}-3,5$ Azidobenzoyl), 7.15 (d, $J=8.1 \mathrm{~Hz}, 3 \mathrm{H}, 3 \times \mathrm{CH}$ Phenyl), $4.33\left(\mathrm{~m}, 1 \mathrm{H}, \mathrm{C}_{\mathrm{a}} \mathrm{H}\right.$ Lysine), 3.03 (q, $J=$ $6.0 \mathrm{~Hz}, 2 \mathrm{H}, \mathrm{C}_{\varepsilon} \mathrm{H}_{2}$ ), 2.04 (t, J = 7.4 Hz, 2H, $\mathrm{C}_{\alpha} \mathrm{H}_{2}$ Butanoyl), $1.83-1.67$ (m, 4H, $\mathrm{C}_{\beta} \mathrm{H}_{2}$ Lysine and $\mathrm{C}_{\beta} \mathrm{H}_{2}$ Butanoyl), $1.47-1.29\left(\mathrm{~m}, 4 \mathrm{H}, \mathrm{C}_{\delta} \mathrm{H}_{2}\right.$ Lysine and $\mathrm{C}_{\gamma} \mathrm{H}_{2}$ Lysine). The signal of $\mathrm{C}_{\gamma} \mathrm{H}_{2}$ Butanoyl overlaps with the DMSO solvent peak. ${ }^{13} \mathrm{C}$-NMR (DMSO- $\left.d_{6}\right) \delta=173.79(\mathrm{C}=\mathrm{O})$, 171.63 (C=O), 165.56 (C=O), 142.40 (C-4 Azidobenzoyl), 141.75 (C-1 Phenyl), 130.50 (C-1 Azidobenzoyl), 129.38 (C-2,6 Azidobenzoyl), 128.27 (2xC Phenyl), 128.23 (2xC Phenyl), 125.70 (1xC Phenyl), 118.81 (C-3,5 Azidobenzoyl), 52.60 ( $\mathrm{C}_{\alpha}$ Lysine), 38.11 ( $\mathrm{C}_{\varepsilon}$ Lysine), 34.85 ( $\mathrm{C}_{\alpha}$ Butanoyl), 34.66 ( $\mathrm{C}_{\mathrm{Y}}$ Butanoyl), 30.19 ( $\mathrm{C}_{\beta}$ Lysine), 28.79 ( $\mathrm{C}_{\delta}$ Lysine), 27.06 ( $\mathrm{C}_{\beta}$ Butanoyl), $23.29\left(\mathrm{C}_{\mathrm{Y}}\right.$ Lysine). MS (ESI+): $\mathrm{m} / \mathrm{z}$ calculated for $\mathrm{C}_{23} \mathrm{H}_{28} \mathrm{~N}_{5} \mathrm{O}_{4}: 438.21[\mathrm{M}+\mathrm{H}]^{+}$, found: 437.91 


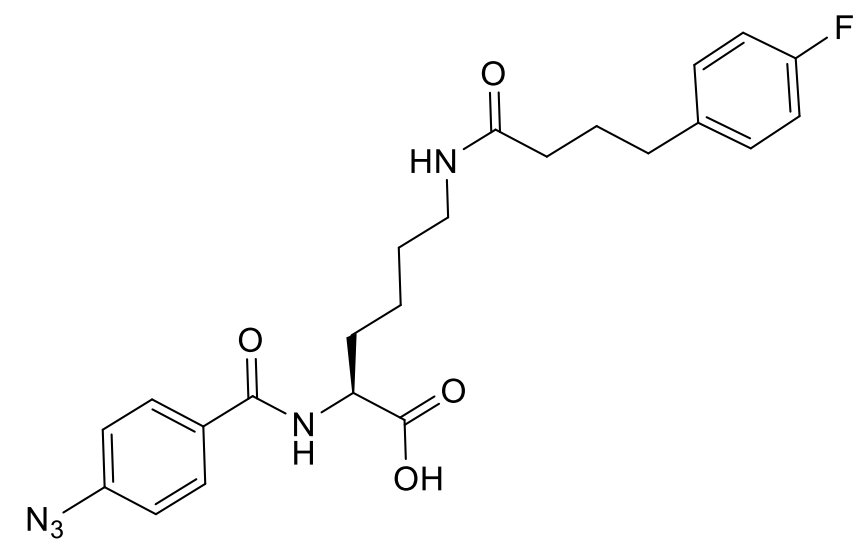

H_25-75_5iso_25min_1 $\mathrm{mL} \_42$ total $\mathrm{t}_{\mathrm{R}}=23.0 \mathrm{~min}$

Compound (S)-1i (12 mg, 38\% overall yield, slightly yellow solid) was synthesised starting from Boc-L-Lysine-OH according to GPs XI, XIV and XII. ${ }^{1} \mathrm{H}-\mathrm{NMR}\left(\mathrm{DMSO}-\mathrm{d}_{6}\right) \delta=12.53$ (br s, $1 \mathrm{H}$, $\mathrm{COOH}$ ), $8.56\left(\mathrm{~d}, J=7.6 \mathrm{~Hz}, 1 \mathrm{H}, \mathrm{N}_{\mathrm{a}} \mathrm{H}\right.$ ), 7.94 (d, $J=8.6 \mathrm{~Hz}, 2 \mathrm{H}, \mathrm{H}-2,6$ Azidobenzoyl), 7.76 (t, $J$ $\left.=5.7 \mathrm{~Hz}, 1 \mathrm{H}, \mathrm{N}_{\varepsilon} \mathrm{H}\right), 7.22-7.15(\mathrm{~m}, 4 \mathrm{H}, \mathrm{H}-2,6$ Fluorophenyl and H-3,5 Azidobenzoyl), 7.07 (t, $J=8.9 \mathrm{~Hz}, 2 \mathrm{H}, \mathrm{H}-3,5$ Fluorophenyl), $4.40-4.27$ (m, 1H, $\mathrm{C}_{\alpha} \mathrm{H}$ Lysine), 3.02 (q, $J=6.3 \mathrm{~Hz}, 2 \mathrm{H}$, $\mathrm{C}_{\varepsilon} \mathrm{H}_{2}$ ), 2.03 (t, $J=7.5 \mathrm{~Hz}, 2 \mathrm{H}, \mathrm{C}_{\alpha} \mathrm{H}_{2}$ Butanoyl), $1.84-1.69\left(\mathrm{~m}, 4 \mathrm{H}, \mathrm{C}_{\beta} \mathrm{H}_{2}\right.$ Lysine and $\mathrm{C}_{\beta} \mathrm{H}_{2}$ Butanoyl), $1.45-1.30\left(\mathrm{~m}, 4 \mathrm{H}, \mathrm{C}_{\delta} \mathrm{H}_{2}\right.$ Lysine and $\mathrm{C}_{\gamma} \mathrm{H}_{2}$ Lysine). The signal of $\mathrm{C}_{\gamma} \mathrm{H}_{2}$ Butanoyl overlaps with DMSO solvent peak. ${ }^{13} \mathrm{C}$-NMR $\left(\mathrm{DMSO}_{-} \mathrm{d}_{6}\right) \delta=173.78(\mathrm{C}=\mathrm{O}), 171.57(\mathrm{C}=\mathrm{O})$, 165.55 (C=O), 160.56 (d, J=240.9 Hz, C-4 Fluorophenyl), 142.40 (C-4 Azidobenzoyl), 137.84 (d, $J=3.1 \mathrm{~Hz}$, C-1 Fluorophenyl), 130.49 (C-1 Azidobenzoyl), 129.97 (d, $J=7.8 \mathrm{~Hz}, \mathrm{C}-2,6$ Fluorophenyl), 129.37 (C-2,6 Azidobenzoyl), 118.81 (C-3,5 Azidobenzoyl), 114.85 (d, $J=20.8$ $\mathrm{Hz}, \mathrm{C}-3,5$ Fluorphenyl), 52.59 ( $\mathrm{C}_{\alpha}$ Lysine), 38.10 ( $\mathrm{C}_{\varepsilon}$ Lysine), 34.70 ( $\mathrm{C}_{\alpha}$ Butanoyl), $33.72\left(\mathrm{C}_{\mathrm{V}}\right.$ Butanoyl), 30.18 ( $\mathrm{C}_{\beta}$ Lysine), 28.78 ( $\mathrm{C}_{\delta}$ Lysine), 27.09 ( $\mathrm{C}_{\beta}$ Butanoyl), 23.29 ( $\mathrm{C}_{\mathrm{Y}}$ Lysine). ${ }^{19} \mathrm{~F}-$ NMR $\left(D M S O-d_{6}\right) \delta=-117.71--177.82\left(m\right.$, Fluorophenyl). MS (ESI $\left.{ }^{+}\right): m / z$ calculated for $\mathrm{C}_{23} \mathrm{H}_{27} \mathrm{FN}_{5} \mathrm{O}_{4}: 456.20[\mathrm{M}+\mathrm{H}]^{+}$, found: 455.82 


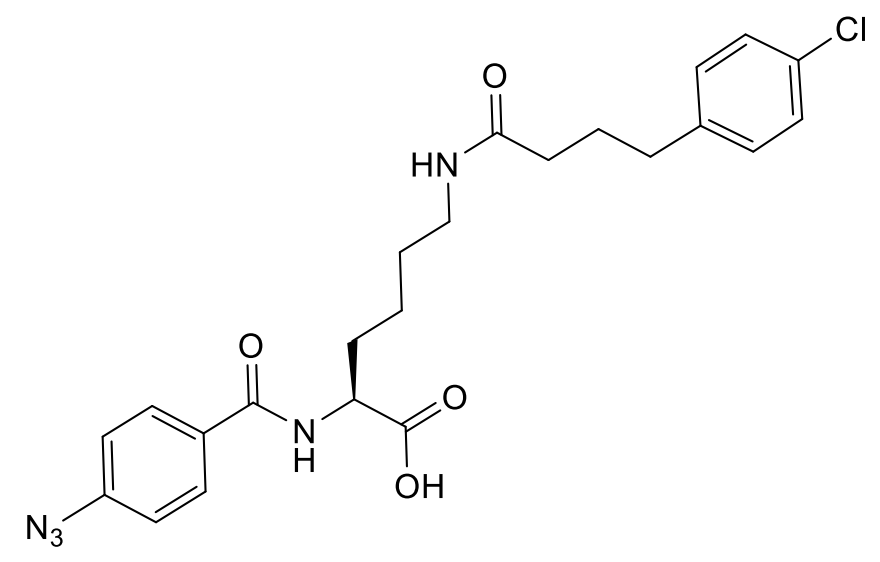

H_25-75_5iso_25min_1 $\mathrm{mL} \_42$ total $\mathrm{t}_{\mathrm{R}}=23.5 \mathrm{~min}$

Compound (S)-1j (12 mg, 19\% overall yield, slightly yellow solid) was synthesised starting from Boc-L-Lysine-OH according to GPs XI, XIV and XII. ${ }^{1} \mathrm{H}-\mathrm{NMR}\left(\mathrm{DMSO}^{-\mathrm{d}_{6}}\right) \delta=12.44(\mathrm{br} \mathrm{s}, 1 \mathrm{H}$, $\mathrm{COOH}$ ), 8.55 (d, $J=7.6 \mathrm{~Hz}, 1 \mathrm{H}, \mathrm{N}_{\mathrm{a}} \mathrm{H}$ ), 7.94 (d, $J=8.7 \mathrm{~Hz}, 2 \mathrm{H}, \mathrm{H}-2,6$ Azidobenzoyl), 7.76 (t, $J$ $=5.6 \mathrm{~Hz}, 1 \mathrm{H}, \mathrm{N}_{\varepsilon} \mathrm{H}$ ), $7.31(\mathrm{~d}, J=8.4 \mathrm{~Hz}, 2 \mathrm{H}, \mathrm{H}-3,5$ Chlorophenyl), 7.19 (dd, $J=8.5,5.8 \mathrm{~Hz}, 4 \mathrm{H}$, $\mathrm{H}-3,5$ Azidobenzoyl and $\mathrm{H}-2,6$ Chlorophenyl), $4.42-4.18$ ( $\mathrm{m}, 1 \mathrm{H}, \mathrm{C}_{\alpha} \mathrm{H}$ Lysine), 3.01 (t, $J=6.3$ $\mathrm{Hz}, 2 \mathrm{H}, \mathrm{C}_{\varepsilon} \mathrm{H}_{2}$ ), $2.03\left(\mathrm{t}, \mathrm{J}=7.4 \mathrm{~Hz}, 2 \mathrm{H}, \mathrm{C}_{\alpha} \mathrm{H}_{2}\right.$ Butanoyl), $1.83-1.68\left(\mathrm{~m}, 4 \mathrm{H}, \mathrm{C}_{\beta} \mathrm{H}_{2}\right.$ Lysine and $\mathrm{C}_{\beta} \mathrm{H}_{2}$ Butanoyl), $1.43-1.30\left(\mathrm{~m}, 4 \mathrm{H}, \mathrm{C}_{\delta} \mathrm{H}_{2}\right.$ Lysine and $\mathrm{C}_{\gamma} \mathrm{H}_{2}$ Lysine). The signal of $\mathrm{C}_{\gamma} \mathrm{H}_{2}$ Butanoyl overlaps with DMSO solvent peak. ${ }^{13} \mathrm{C}-\mathrm{NMR}\left(\mathrm{DMSO}_{\mathrm{d}}\right) \delta=173.78(\mathrm{C}=\mathrm{O}), 171.52(\mathrm{C}=\mathrm{O})$, 165.54 (C=O), 142.39 (C-4 Azidobenzoyl), 140.75 (C-1 Chlorophenyl), 130.39 (d, J=19.3 Hz, C-4 Chlorophenyl), 130.16 (C-2,6 Chlorophenyl), 129.36 (C-2,6 Azidobenzoyl), 128.13 (C-3,5 Chlorphenyl), 118.81 (C-3,5 Azidobenzoyl), 52.60 ( $\mathrm{C}_{\alpha}$ Lysine), 38.11 ( $\mathrm{C}_{\varepsilon}$ Lysine), 34.65 ( $\mathrm{C}_{\alpha}$ Butanoyl), 33.84 ( $\mathrm{C}_{\mathrm{Y}}$ Butanoyl), 30.20 ( $\mathrm{C}_{\beta}$ Lysine), 28.77 ( $\mathrm{C}_{\delta}$ Lysine), 26.83 ( $\mathrm{C}_{\beta}$ Butanoyl), $23.29\left(\mathrm{C}_{\mathrm{Y}}\right.$ Lysine). MS $\left(\mathrm{ESI}^{+}\right): \mathrm{m} / \mathrm{z}$ calculated for $\mathrm{C}_{23} \mathrm{H}_{27} \mathrm{CIN}_{5} \mathrm{O}_{4}: 472.17[\mathrm{M}+\mathrm{H}]^{+}$, found: 471.83 


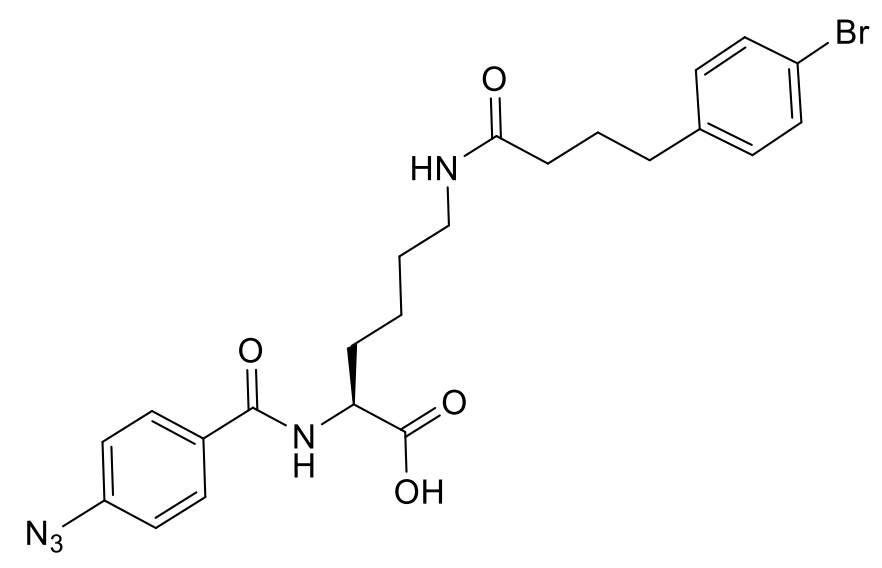

H_25-75_5iso_25min_1 $\mathrm{mL} \_42$ total $\mathrm{t}_{\mathrm{R}}=25.4 \mathrm{~min}$

Compound (S)-1k (34 mg, 52\% overall yield, slightly yellow solid) was synthesised starting from Boc-L-Lysine-OH according to GPs XI, XIV and XII. ${ }^{1} \mathbf{H}-\mathbf{N M R}\left(600 \mathrm{MHz}, \mathrm{DMSO}-\mathrm{d}_{6}\right) \delta=$ $8.56\left(\mathrm{~d}, J=7.6 \mathrm{~Hz}, 1 \mathrm{H}, \mathrm{N}_{\mathrm{a}} \mathrm{H}\right.$ ), 7.94 (d, $J=8.6 \mathrm{~Hz}, 2 \mathrm{H}, \mathrm{H}-2,6$ Azidobenzoyl), 7.76 (t, $J=5.7 \mathrm{~Hz}$, $1 \mathrm{H}, \mathrm{N}_{\varepsilon} \mathrm{H}$ ), $7.44(\mathrm{~d}, J=8.3 \mathrm{~Hz}, 2 \mathrm{H}, \mathrm{H}-3,5$ Bromophenyl), 7.19 (d, J = 8.6 Hz, 2H, H-3,5 Azidobenzoyl), 7.12 (d, $J=8.3 \mathrm{~Hz}, 2 \mathrm{H}, \mathrm{H}-2,6$ Bromophenyl), $4.37-4.26$ (m, 1H, $\mathrm{C}_{\alpha} \mathrm{H}$ Lysine), 3.02 (q, $J=6.6 \mathrm{~Hz}, 2 \mathrm{H}, \mathrm{C}_{\varepsilon} \mathrm{H}_{2}$ ), 2.02 (t, $J=7.4 \mathrm{~Hz}, 2 \mathrm{H}, \mathrm{C}_{\mathrm{a}} \mathrm{H}_{2}$ Butanoyl), $1.87-1.66(\mathrm{~m}, 4 \mathrm{H}$, $\mathrm{C}_{\beta} \mathrm{H}_{2}$ Lysine and $\mathrm{C}_{\beta} \mathrm{H}_{2}$ Butanoyl), $1.47-1.28\left(\mathrm{~m}, 4 \mathrm{H}, \mathrm{C}_{\delta} \mathrm{H}_{2}\right.$ Lysine and $\mathrm{C}_{\gamma} \mathrm{H}_{2}$ Lysine). The signal of $\mathrm{C}_{\mathrm{y}} \mathrm{H}_{2}$ Butanoyl overlaps with DMSO solvent peak. ${ }^{13} \mathrm{C}-\mathrm{NMR}\left(151 \mathrm{MHz}\right.$, DMSO-d $\left.\mathrm{d}_{6}\right) \delta=173.79$ $(\mathrm{C}=\mathrm{O}), 171.53(\mathrm{C}=\mathrm{O}), 165.56(\mathrm{C}=\mathrm{O}), 142.41$ (C-4 Azidobenzoyl), 141.18 (C-1 Bromophenyl), 131.06 (C-3,5 Bromophenyl), 130.60 (C-2,6 Bromophenyl), 130.49 (C-1 Azidobenzoyl), 129.38 (C-2,6 Azidobenzoyl), 118.81 (C-3,5 Azidobenzoyl), 118.73 (C-4 Bromophenyl), 52.60 ( $\mathrm{C}_{\alpha}$ Lysine), 38.12 ( $\mathrm{C}_{\varepsilon}$ Lysine), 34.65 ( $\mathrm{C}_{\alpha}$ Butanoyl), 33.90 ( $\mathrm{C}_{\mathrm{\gamma}}$ Butanoyl), 30.19 ( $\mathrm{C}_{\beta}$ Lysine), 28.78 ( $\mathrm{C}_{\delta}$ Lysine), 26.78 ( $\mathrm{C}_{\beta}$ Butanoyl), $23.30\left(\mathrm{C}_{\mathrm{\gamma}}\right.$ Lysine). MS $\left(\mathrm{ESI}^{+}\right): \mathrm{m} / \mathrm{z}$ calculated for $\mathrm{C}_{23} \mathrm{H}_{27} \mathrm{BrN}_{5} \mathrm{O}_{4}: 516.12[\mathrm{M}+\mathrm{H}]^{+}$, found: 515.76 


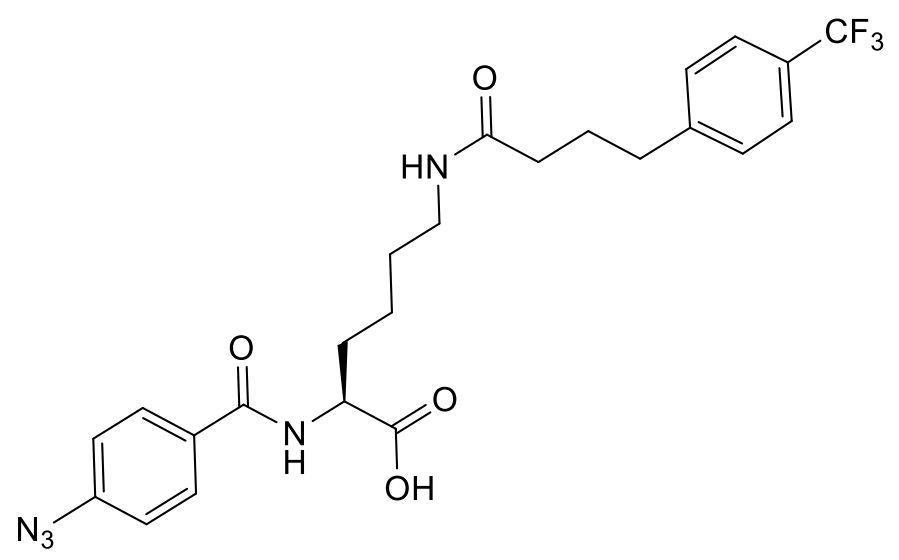

H_25-75_5iso_25min_1 $\mathrm{mL} \_42$ total $\mathrm{t}_{\mathrm{R}}=26.7 \mathrm{~min}$

Compound (S)-1I (23 mg, 39\% overall yield, white solid) was synthesised starting from Boc-LLysine-OH according to GPs XI, XIV and XII. ' ${ }^{1} \mathrm{H}-\mathrm{NMR}$ (DMSO- $\left.\mathrm{d}_{6}\right) \delta=12.55$ (br s, $1 \mathrm{H}, \mathrm{COOH}$ ), $8.56\left(\mathrm{~d}, J=7.6 \mathrm{~Hz}, 1 \mathrm{H}, \mathrm{N}_{\mathrm{a}} \mathrm{H}\right.$ ), 7.94 (d, $J=8.6 \mathrm{~Hz}, 2 \mathrm{H}, \mathrm{H}-2,6$ Azidobenzoyl), 7.78 (t, $J=5.7 \mathrm{~Hz}$, $1 \mathrm{H}, \mathrm{N}_{\varepsilon} \mathrm{H}$ ), 7.61 (d, $J=7.7 \mathrm{~Hz}, 2 \mathrm{H}, \mathrm{H}-3,5$ Trifluoromethylphenyl), 7.39 (d, $J=7.9 \mathrm{~Hz}, 2 \mathrm{H}, \mathrm{H}-2,6$ Trifluoromethylphenyl), $7.19\left(\mathrm{~d}, J=8.7 \mathrm{~Hz}, 2 \mathrm{H}, \mathrm{H}-3,5\right.$ Azidobenzoyl), $4.38-4.28\left(\mathrm{~m}, 1 \mathrm{H}, \mathrm{C}_{\mathrm{a}} \mathrm{H}\right.$ Lysine), 3.03 (q, $J=6.6 \mathrm{~Hz}, 2 \mathrm{H}, \mathrm{C}_{\varepsilon} \mathrm{H}_{2}$ ), 2.61 (t, $J=7.6 \mathrm{~Hz}, 2 \mathrm{H}, \mathrm{C}_{\mathrm{\gamma}} \mathrm{H}_{2}$ Butanoyl), 2.05 (t, $J=7.4$ $\mathrm{Hz}, 2 \mathrm{H}, \mathrm{C}_{\alpha} \mathrm{H}_{2}$ Butanoyl), $1.87-1.70\left(\mathrm{~m}, 4 \mathrm{H}, \mathrm{C}_{\beta} \mathrm{H}_{2}\right.$ Lysine and $\mathrm{C}_{\beta} \mathrm{H}_{2}$ Butanoyl), $1.53-1.28(\mathrm{~m}$, $4 \mathrm{H}, \mathrm{C}_{\delta} \mathrm{H}_{2}$ Lysine and $\mathrm{C}_{\mathrm{\gamma}} \mathrm{H}_{2}$ Lysine). ${ }^{13} \mathrm{C}-\mathrm{NMR}$ (DMSO- $\left.\mathrm{d}_{6}\right) \delta=173.78(\mathrm{C}=\mathrm{O}), 171.45(\mathrm{C}=\mathrm{O})$, 165.55 (C=O), 146.75 (C-1 Trifluoromethylphenyl), 142.39 (C-4 Azidobenzoyl), 130.48 (C-1 Azidobenzoyl), 129.37 (C-2,6 Azidobenzoyl), 129.11 (C-2,6 Trifluoromethylphenyl), 126.56 (q, $J=32.0 \mathrm{~Hz}, \mathrm{C}-4$ Trifluoromethylphenyl), 125.05 (q, $J=3.8 \mathrm{~Hz}, \mathrm{C}-3,5$ Trifluoromethylphenyl), 124.45 (q, $\left.J=273.0 \mathrm{~Hz}, \mathrm{CF}_{3}\right) 118.80$ (C-3,5 Azidobenzoyl), 52.59 ( $\mathrm{C}_{\alpha}$ Lysine), $38.12\left(\mathrm{C}_{\varepsilon}\right.$ Lysine), 34.62 ( $\mathrm{C}_{\alpha}$ Butanoyl), 34.32 ( $\mathrm{C}_{\mathrm{Y}}$ Butanoyl), 30.19 ( $\mathrm{C}_{\beta}$ Lysine), 28.77 ( $\mathrm{C}_{\delta}$ Lysine), 26.59 ( $\mathrm{C}_{\beta}$ Butanoyl), 23.30 ( $\mathrm{C}_{\mathrm{\gamma}}$ Lysine). ${ }^{19} \mathrm{~F}-\mathrm{NMR}$ MS (ESI+): $\mathrm{m} / \mathrm{z}$ calculated for $\mathrm{C}_{24} \mathrm{H}_{27} \mathrm{~F}_{3} \mathrm{~N}_{5} \mathrm{O}_{4}: 506.20$ $[\mathrm{M}+\mathrm{H}]^{+}$, found: 505.85 


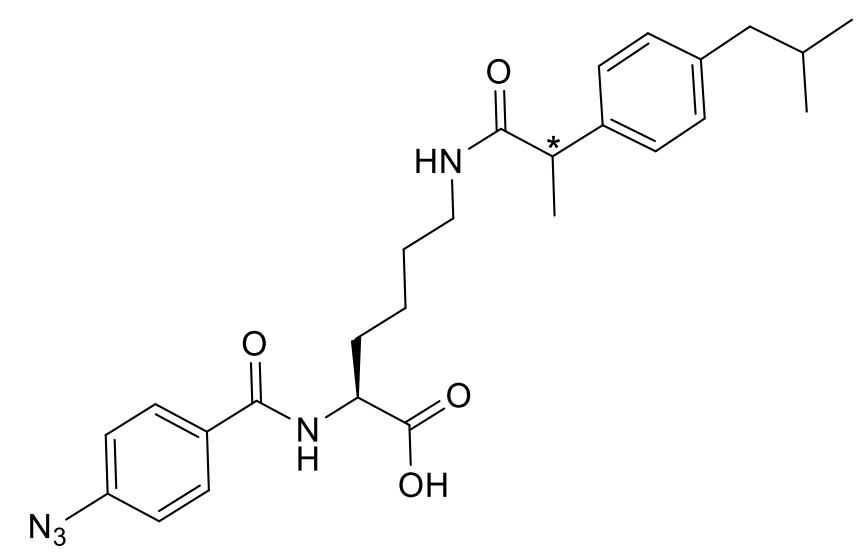

\section{H_25-75_5iso_25min_1 $\mathrm{mL}$ 42total $t_{R}=29.5 \mathrm{~min}$}

Compound (S)-1m (46 mg, 34\% overall yield, slightly yellow solid) was synthesised starting from Boc-L-Lysine-OH according to GPs XI, XIV and XII. ${ }^{1} \mathbf{H}-\mathbf{N M R}\left(\mathrm{CDCl}_{3}\right) \delta=7.95-7.85(\mathrm{~m}$, $2 \mathrm{H}, \mathrm{H}-3,5$ Azidobenzoyl and $\mathrm{N}_{\mathrm{a}} \mathrm{H}$ ), 7.07 (dd, $J=8.1,3.0 \mathrm{~Hz}, 1 \mathrm{H}, \mathrm{H}-2,6$ Azidobenzoyl), $6.98-$ $6.87\left(\mathrm{~m}, 4 \mathrm{H}, \mathrm{H}-2,3,5,6\right.$ Isobutylphenyl), $5.51-5.41\left(\mathrm{~m}, 1 \mathrm{H}, \mathrm{N}_{\varepsilon} \mathrm{H}\right), 5.03-4.94\left(\mathrm{~m}, 1 \mathrm{H}, \mathrm{C}_{\mathrm{a}} \mathrm{H}\right.$ Lysine), $3.65-3.47\left(\mathrm{~m}, 2 \mathrm{H}, 1 \times \mathrm{H} \mathrm{C}_{\varepsilon} \mathrm{HH}\right.$ Lysine and $\left.\mathrm{CH}_{3}-\mathrm{CH}-\mathrm{CO}\right), 3.11-2.96\left(\mathrm{~m}, 1 \mathrm{H}, \mathrm{C}_{\varepsilon} \mathrm{HH}\right.$ Lysine), $2.42-2.29\left(\mathrm{~m}, 1 \mathrm{H}, \mathrm{CH}_{2}\right.$ Isobutyl), $2.04-1.84$ ( $\mathrm{m}, 2 \mathrm{H}, \mathrm{C}_{\beta} \mathrm{H}_{2}$ Lysine), $1.84-1.67$ (m, $1 \mathrm{H}, \mathrm{CH}$ Isobutyl), $1.52-1.23\left(\mathrm{~m}, \mathrm{CH}_{3}\right.$ Isopropionyl, $\mathrm{C}_{\mathrm{\gamma}} \mathrm{H}_{2}$ Lysine and $\mathrm{C}_{\delta} \mathrm{H}_{2}$ Lysine), 0.85 (t, $\mathrm{J}=$ $6.5 \mathrm{~Hz}, 6 \mathrm{H}, 2 \times \mathrm{CH}_{3}$ Isobutyl). ${ }^{13} \mathrm{C}-\mathrm{NMR}\left(\mathrm{CDCl}_{3}\right) \delta=177.15 / 176.66(\mathrm{C}=\mathrm{O}), 175.06(\mathrm{C}=\mathrm{O})$,

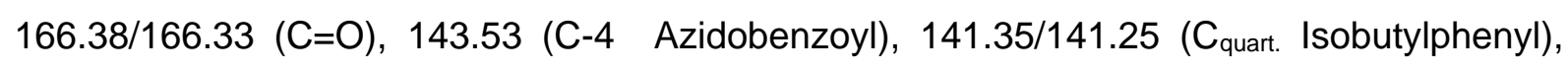

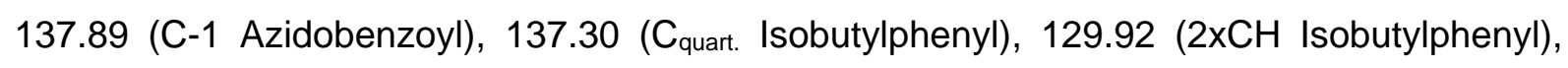

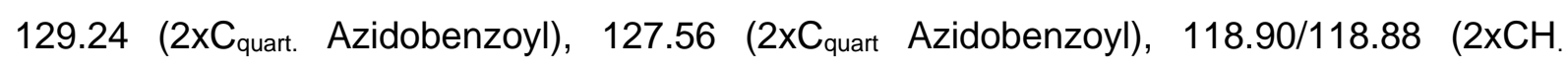
Isobutylphenyl), 52.56/52.51 ( $\mathrm{C}_{\alpha}$ Lysine $), 46.83\left(\mathrm{CH}_{3}-\mathrm{CH}-\mathrm{CO}\right), 45.1\left(\mathrm{CH}_{2}\right.$ Isobutyl), 38.47/38.30 ( $\mathrm{C}_{\varepsilon}$ Lysine), 31.17/31.03 $\left(\mathrm{C}_{\beta} \mathrm{H}_{2}\right.$ Lysine), $30.2\left(\mathrm{CH}\right.$ Isobutyl), 29.66/29.31 $\left(\mathrm{C}_{\delta} \mathrm{H}_{2}\right.$ Lysine $), 22.46 / 22.45 \quad\left(\mathrm{CH}_{3}\right.$ Isobutyl $), 22.00 / 21.78 \quad\left(\mathrm{C}_{\mathrm{\gamma}} \mathrm{H}_{2}\right.$ Lysine $), 18.22 / 18.11 \quad\left(\mathrm{CH}_{3}\right.$ Isopropionyl). Compound (S)-1 $\mathrm{m}$ was obtained as a mixture of two diastereomers with the ratio of $1: 1$. Thus, the ${ }^{1} \mathrm{H}$ - and ${ }^{13} \mathrm{C}$-NMR signals are partially doubled; $\mathrm{MS}\left(\mathrm{ESI}^{+}\right): \mathrm{m} / \mathrm{z}$ calculated for $\mathrm{C}_{26} \mathrm{H}_{34} \mathrm{~N}_{5} \mathrm{O}_{4}: 480.26[\mathrm{M}+\mathrm{H}]^{+}$, found: 479.93 


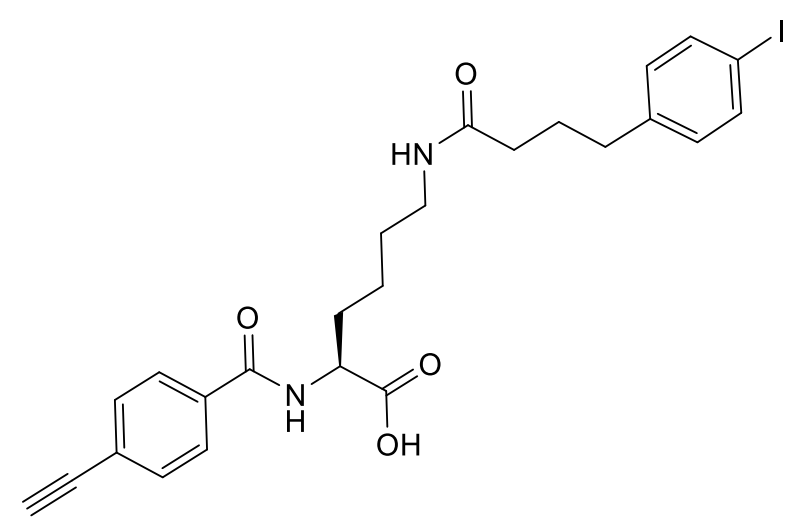

H_25-75_5iso_25min_1 $\mathrm{mL} \_42$ total $\mathrm{t}_{\mathrm{R}}=26.2 \mathrm{~min}$

Compound (S)-1n (12 mg, 47\% overall yield, slightly yellow solid) was synthesised starting from Boc-L-Lysine-OH according to GPs XI, XIV and XII. ${ }^{1} \mathrm{H}-\mathrm{NMR}\left(\mathrm{DMSO}^{\left.-\mathrm{d}_{6}\right)} \delta=12.56\right.$ (br s, $1 \mathrm{H}, \mathrm{COOH}$ ), $8.64\left(\mathrm{~d}, J=7.7 \mathrm{~Hz}, 1 \mathrm{H}, \mathrm{N}_{\mathrm{a}} \mathrm{H}\right.$ ), $7.89(\mathrm{~d}, J=8.4 \mathrm{~Hz}, 2 \mathrm{H}, \mathrm{H}-2,6$ Ethynylbenzoyl), 7.76 (t, $J=5.7 \mathrm{~Hz}, 1 \mathrm{H}, \mathrm{N}_{\varepsilon} \mathrm{H}$ ), 7.61 (d, $J=8.2 \mathrm{~Hz}, 2 \mathrm{H}, \mathrm{H}-3,5$ lodophenyl), 7.57 (d, J = 8.4 Hz, 2H, H3,5 Ethynylbenzoyl), 6.98 (d, $J=8.3 \mathrm{~Hz}, 2 \mathrm{H}, \mathrm{H}-2,6$ lodophenyl), 4.38 (s, $1 \mathrm{H}, \mathrm{CH}$ Ethynyl), 4.38 - $4.28\left(\mathrm{~m}, 1 \mathrm{H}, \mathrm{C}_{\alpha} \mathrm{H}\right.$ Lysine), 3.02 (q, $J=6.1 \mathrm{~Hz}, 2 \mathrm{H}, \mathrm{C}_{\varepsilon} \mathrm{H}_{2}$ ), 2.02 (t, $J=7.4 \mathrm{~Hz}, 2 \mathrm{H}, \mathrm{C}_{\alpha} \mathrm{H}_{2}$ Butanoyl), $1.86-1.67\left(\mathrm{~m}, 4 \mathrm{H}, \mathrm{C}_{\beta} \mathrm{H}_{2}\right.$ Lysine and $\mathrm{C}_{\beta} \mathrm{H}_{2}$ Butanoyl), $1.44-1.28\left(\mathrm{~m}, 4 \mathrm{H}, \mathrm{C}_{\delta} \mathrm{H}_{2}\right.$ Lysine and $\mathrm{C}_{\mathrm{\gamma}} \mathrm{H}_{2}$ Lysine). The signal of $\mathrm{C}_{\mathrm{\gamma}} \mathrm{H}_{2}$ Butanoyl overlaps with DMSO solvent peak. ${ }^{13} \mathrm{C}$ NMR $\left(D M S O-d_{6}\right) \delta=173.66(C=O), 171.52(C=O), 165.73(\mathrm{C}=0), 141.55$ (C-1 lodophenyl), 136.94 (C-3,5 lodophenyl), 134.00 (C-1 Ethynylbenzoyl), 131.56 (C-3,5 Ethynylbenzoyl), 130.84 (C-2,6 lodophenyl), 127.73 (C-2,6 Ethynylbenzoyl), 124.55 (C-4 Ethynylbenzoyl), 91.23 (C-4 lodophenyl), 82.87 (C Ethynyl), 52.63 ( $\mathrm{C}_{\alpha}$ Lysine), 38.10 ( $\mathrm{C}_{\varepsilon}$ Lysine), 34.65 ( $\mathrm{C}_{\alpha}$ Butanoyl), 34.02 ( $\mathrm{C}_{\mathrm{Y}}$ Butanoyl), 30.15 ( $\mathrm{C}_{\beta}$ Lysine), 28.76 ( $\mathrm{C}_{\delta}$ Lysine), 26.75 ( $\mathrm{C}_{\beta}$ Butanoyl), $23.27\left(\mathrm{C}_{\mathrm{Y}}\right.$ Lysine). The signal of $\mathrm{CH}$ Ethynyl is not visible. $\mathrm{MS}\left(\mathrm{ESI}^{+}\right): \mathrm{m} / \mathrm{z}$ calculated for $\mathrm{C}_{25} \mathrm{H}_{28} \mathrm{IN}_{2} \mathrm{O}_{4}: 547.11[\mathrm{M}+\mathrm{H}]^{+}$, found: 546.68 


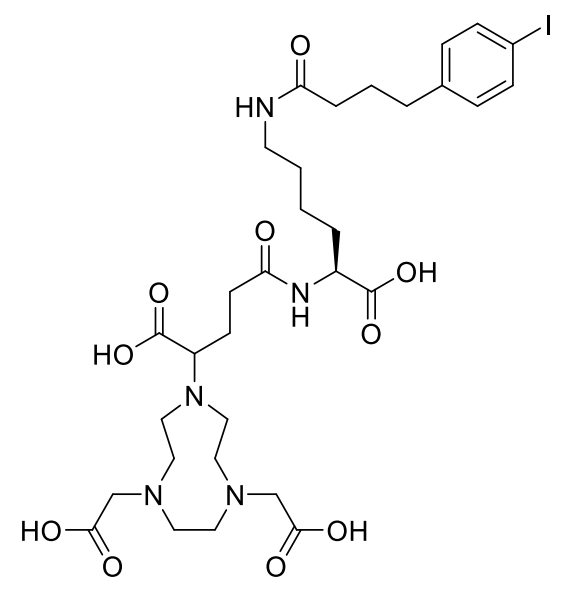

H_25-75_5iso_25min_1 $\mathrm{mL} \_42$ total $\mathrm{t}_{\mathrm{R}}=16.5 \mathrm{~min}$

Compound (S)-10 (18 mg, 34\% overall yield, white solid) was synthesised starting from BocL-Lysine-OH according to GPs XI, XIV and XI using NODAGA-tris(t-Bu ester). After coupling, concentrated $\mathrm{H}_{3} \mathrm{PO}_{4}$ (85\%) was added 1:1 to the aqueous solution and stirred for $3 \mathrm{~h}$ at room temperature to remove the tert-Butyl groups. ${ }^{1} \mathrm{H}-\mathrm{NMR}\left(\mathrm{DMSO}_{-} \mathrm{d}_{6}\right) \delta=12.36(\mathrm{br} \mathrm{s}, 4 \mathrm{H}$, $4 \times \mathrm{COOH}$ ), $8.05\left(\mathrm{~d}, J=7.7 \mathrm{~Hz}, 1 \mathrm{H}, \mathrm{N}_{\alpha} \mathrm{H}\right), 7.77\left(\mathrm{t}, J=5.6 \mathrm{~Hz}, 1 \mathrm{H}, \mathrm{N}_{\varepsilon} \mathrm{H}\right), 7.62(\mathrm{~d}, J=8.2 \mathrm{~Hz}, 2 \mathrm{H}$, $\mathrm{H}-3,5$ lodophenyl), 7.00 (d, J=8.2 Hz, 2H, H-2,6 lodophenyl), 4.17- 4.07 (m, 1H, Ca H Lysine), $3.97-3.63\left(\mathrm{~m}, 4 \mathrm{H}, 2 \mathrm{xNCH} \mathrm{TACN}_{2}, 3.46\right.$ (t, $J=7.3 \mathrm{~Hz}, 1 \mathrm{H}, \mathrm{NCH}$ TACN), $3.25-2.71(\mathrm{~m}, 14 \mathrm{H}$, $6 x \mathrm{CH}_{2}$ TACN and $\mathrm{C}_{\varepsilon} \mathrm{H}_{2}$ Lysine), 2.27 (t, $J=7.5 \mathrm{~Hz}, 2 \mathrm{H}, \mathrm{C}_{\alpha} \mathrm{H}_{2}$ Glutamoyl), 2.03 (q, $J=6.4 \mathrm{~Hz}$, $2 \mathrm{H}, \mathrm{C}_{\alpha} \mathrm{H}_{2}$ Butanoyl), 1.96 (dd, $J=14.4,7.5 \mathrm{~Hz}, 1 \mathrm{H}, \mathrm{C}_{\beta} H \mathrm{H}$ Glutamoyl), 1.85 (p, J=7.4 Hz, $1 \mathrm{H}$, $\mathrm{C}_{\beta} \mathrm{H} H$ Glutamoyl), $1.75\left(\mathrm{p}, J=7.7 \mathrm{~Hz}, 2 \mathrm{H}\right.$, and $\mathrm{C}_{\beta} \mathrm{H}_{2}$ Butanoyl), $1.66\left(\mathrm{q}, J=7.4 \mathrm{~Hz}, 1 \mathrm{H}, \mathrm{C}_{\beta} H \mathrm{H}\right.$ Lysine), $1.63-1.50$ (m, 1H, $\mathrm{C}_{\beta} \mathrm{H} H$ Lysine), $1.41-1.33$ (m, 2H, $\mathrm{C}_{\delta} \mathrm{H}_{2}$ Lysine), $1.32-1.21$ (m, $2 \mathrm{H}, \mathrm{C}_{\mathrm{\gamma}} \mathrm{H}_{2}$ Lysine). The signal of $\mathrm{C}_{\mathrm{\gamma}} \mathrm{H}_{2}$ Butanoyl overlaps with DMSO solvent peak. ${ }^{13} \mathrm{C}-\mathrm{NMR}$ $\left(\right.$ DMSO- $\left.d_{6}\right) \delta=173.79(\mathrm{C}=\mathrm{O}), 173.47(\mathrm{C}=\mathrm{O}), 171.87(\mathrm{C}=\mathrm{O}), 171.55(\mathrm{C}=\mathrm{O}), 141.58(\mathrm{C}-1$ lodophenyl), 136.96 (C-3,5 lodophenyl), 130.86 (C-2,6 lodophenyl), 91.27 (C-4 lodophenyl), 62.85 ( $\mathrm{NCH}$ TACN), 54.4 ( $\mathrm{NCH}_{2}$ TACN), 54.2 ( $\mathrm{NCH}_{2}$ TACN), 51.80 (C $\alpha$ Lysine), 54-48 $\left(6 \times \mathrm{CH}_{2}\right.$ TACN), 38.15 ( $\mathrm{C}_{\varepsilon}$ Lysine), 34.63 ( $\mathrm{C}_{\alpha}$ Butanoyl), 34.03 ( $\mathrm{C}_{\mathrm{Y}}$ Butanoyl), 31.73 ( $\mathrm{C}_{\alpha}$ Glutamoyl), 30.63 ( $\mathrm{C}_{\beta}$ Lysine), 28.78 ( $\mathrm{C}_{\delta}$ Lysine), 26.74 ( $\mathrm{C}_{\beta}$ Butanoyl), 25.08 ( $\mathrm{C}_{\beta}$ Glutamoyl), 22.91( $\mathrm{C}_{\mathrm{Y}}$ Lysine). Signal of $1 \times \mathrm{C}=\mathrm{O}$ is not visible. $\mathrm{MS}\left(\mathrm{ESI}^{+}\right): \mathrm{m} / \mathrm{z}$ calculated for $\mathrm{C}_{31} \mathrm{H}_{44} \mathrm{FelN}_{5} \mathrm{O}_{10}: 829.15$ $[\mathrm{M}-2 \mathrm{H}+\mathrm{Fe}]^{+}$, found: $828.85\left[\mathrm{M}-2 \mathrm{H}^{+}+\mathrm{Fe}^{3+}\right]^{+}$. 


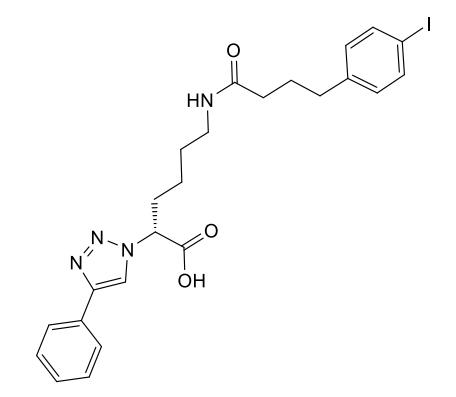

H_25-75_5iso_25min_1 $\mathrm{mL} \_42$ total $\mathrm{t}_{\mathrm{R}}=27.1 \mathrm{~min}$

Compound $(\boldsymbol{R}) \mathbf{- 1 p}$ (92 mg, 65\% overall yield, white solid) was synthesised starting from $N^{\alpha_{-}}$ azido- $N^{\varepsilon}\left(\right.$ Boc)-D-lysine. $\quad N^{\alpha}$-azido- $N^{\varepsilon}($ Boc $)$-D-lysine $\quad(70 \mathrm{mg}, \quad 0.26 \mathrm{mmol}), \quad \mathrm{CuSO}_{4}{ }^{*} 5 \mathrm{H}_{2} \mathrm{O}$ (5 mol\%) and THPTA (5 mol\%) were dissolved in $1.5 \mathrm{~mL}$ water. Phenylacetylen $(31 \mu \mathrm{L}$, $0.28 \mathrm{mmol}, 1.1 \mathrm{eq}$.) in aceton (6.4 mL) was added. Ascorbinic acid (22.6 mg, $0.13 \mathrm{mmol}, 0.5$ eq.) was added and the mixture was stirred for $1 \mathrm{~h}$. Aceton was removed under reduced pressure and the aqueous solution was extracted $\mathrm{CH}_{2} \mathrm{Cl}_{2}(2 \times 5 \mathrm{~mL})$. The combined organic phase was evaporated. The residue was dissolved in TFA: $\mathrm{CH}_{2} \mathrm{Cl}_{2}$ 1:1 (v/v, $\left.4 \mathrm{~mL}\right)$ and stirred for $1 \mathrm{~h}$. All volatiles were removed under reduced pressure. The residue was dissolved in dioxan (6 mL). After addition of DIPEA (300 $\mu \mathrm{L}, 1.03 \mathrm{mmol}, 4$ eq.) and IPB-NHS (105.0 mg, $0.27 \mathrm{mmol}, 1.05 \mathrm{Eq}$.) the solution was stirred for $2 \mathrm{~h}$. Dioxan was removed under reduced pressure before adjusting the $\mathrm{pH}$ to $<3$ by addition of $1 \mathrm{~N} \mathrm{HCl}$. The aqueous solution was extracted with ethyl acetate $(2 \times 5 \mathrm{~mL})$. Purification was done by RP-HPLC. ${ }^{1} \mathbf{H}-\mathbf{N M R}\left(\mathrm{CDCl}_{3}\right) \delta$ $=8.15\left(\mathrm{~s}, 1 \mathrm{H}, \mathrm{C}_{4} \mathrm{H}\right.$ Triazol), $7.77(\mathrm{~d}, J=6.9 \mathrm{~Hz}, 2 \mathrm{H}, \mathrm{H}-2,6$ Phenyl), $7.52(\mathrm{~d}, J=8.2 \mathrm{~Hz}, 2 \mathrm{H}, \mathrm{H}-$ 3,5 lodophenyl), 7.36 (t, $J=7.2 \mathrm{~Hz}, 2 \mathrm{H}, \mathrm{H}-3,5$ Phenyl), 7.29 (t, $J=7.3 \mathrm{~Hz}, 1 \mathrm{H}, \mathrm{H}-4$ Phenyl), 6.81 (d, $J=8.3 \mathrm{~Hz}, 2 \mathrm{H}, \mathrm{H}-2,6$ lodophenyl), 5.94 (s, $1 \mathrm{H}, \mathrm{N}_{\varepsilon} \mathrm{H}$ ), 5.42 (dd, $J=9.8,5.1 \mathrm{~Hz}, 1 \mathrm{H}$, $\mathrm{C}_{\mathrm{a}} \mathrm{H}$ Lysine), 3.21 (dp, $J=20.0,6.7 \mathrm{~Hz}, 2 \mathrm{H}, \mathrm{C}_{\varepsilon} \mathrm{H}_{2}$ Lysine), 2.44 (t, $J=7.5 \mathrm{~Hz}, 2 \mathrm{H}, \mathrm{C}_{\mathrm{\gamma}} \mathrm{H}_{2}$ Butanoyl), $2.36-2.17$ (m, 2H, $\mathrm{C}_{\alpha} \mathrm{H}_{2}$ Butanoyl), 2.09 (t, $J=7.5 \mathrm{~Hz}, 2 \mathrm{H}, \mathrm{C}_{\beta} \mathrm{H}_{2}$ Butanoyl), 1.81 (quint., $J=7.6 \mathrm{~Hz}, 2 \mathrm{H}, \mathrm{C}_{\beta} \mathrm{H}_{2}$ Lysine), 1.50 (quint., $J=7.1 \mathrm{~Hz}, 2 \mathrm{H}, \mathrm{C}_{\delta} \mathrm{H}_{2}$ Lysine), $1.42-1.28$ (m, $1 \mathrm{H}, \mathrm{C}_{\mathrm{\gamma}} \mathrm{H} H$ Lysine), $1.26-1.13\left(\mathrm{~m}, 1 \mathrm{H}, \mathrm{C}_{\mathrm{\gamma}} H \mathrm{H}\right.$ Lysine). ${ }^{13} \mathrm{C}-\mathrm{NMR}\left(\mathrm{CDCl}_{3}\right) \delta=174.31(\mathrm{C}=\mathrm{O})$, 140.96 (C-1 lodophenyl), 137.57 (C-3,5 lodophenyl, 130.70 (C-2,6 lodophenyl, 129.11 (C-3,5 Phenyl), 128.88 (C-4 Phenyl), 126.00 (C-2,6 Phenyl), 120.46 (C-5 Triazol), 91.22 (C-4 lodophenyl), 63.29 ( $\mathrm{C}_{\alpha}$ Lysine), 38.80 ( $\mathrm{C}_{\varepsilon}$ Lysine), 35.74 ( $\mathrm{C}_{\beta}$ Butanoyl), 34.67 ( $\mathrm{C}_{\mathrm{Y}}$ Butanoyl), 31.85 ( $\mathrm{C}_{\alpha}$ Butanoyl), 28.50 ( $\mathrm{C}_{\delta}$ Lysine), 26.95 ( $\mathrm{C}_{\beta}$ Lysine), 22.55 ( $\mathrm{C}_{\mathrm{Y}}$ Lysine). The signals of $1 \times \mathrm{C}=\mathrm{O}$ and $\mathrm{C}-4$ Triazol were too low to be determined. $\mathrm{MS}\left(\mathrm{ESI}^{+}\right): \mathrm{m} / \mathrm{z}$ calculated for $\mathrm{C}_{24} \mathrm{H}_{28} \mathrm{IN}_{4} \mathrm{O}_{3}: 547.12[\mathrm{M}+\mathrm{H}]^{+}$, found: 547.08 


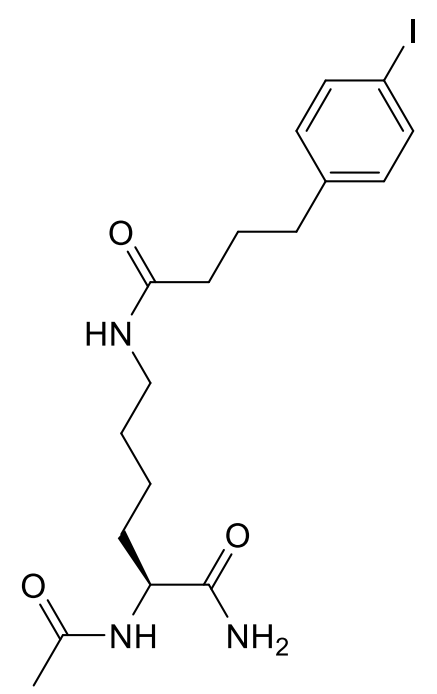

H_25-75_5iso_25min_1 $\mathrm{mL} \_42$ total $\mathrm{t}_{\mathrm{R}}=18.2 \mathrm{~min}$

Compound (S)-2a (33 mg, 26\% overall yield, yellow solid) was synthesised starting from AllocD-Lys(Fmoc)-OH according to GPs II, III, VI, VII, IV and VIIIb. ${ }^{1} \mathbf{H}-\mathbf{N M R}$ (DMSO-d 6 ) $\delta=7.85$ (d, $J=8.1 \mathrm{~Hz}, 1 \mathrm{H}, \mathrm{N}_{\mathrm{a}} \mathrm{H}$ ), $7.73\left(\mathrm{t}, J=5.6 \mathrm{~Hz}, 1 \mathrm{H}, \mathrm{N}_{\varepsilon} \mathrm{H}\right.$ ), $7.62(\mathrm{~d}, J=8.2 \mathrm{~Hz}, 2 \mathrm{H}, \mathrm{H}-3,5$ lodophenyl), $7.30(\mathrm{~s}, 1 \mathrm{H}, \mathrm{NHH}), 7.01$ (d, J=8.2 Hz, 2H, H-2,6 lodophenyl), $6.92(\mathrm{~s}, 1 \mathrm{H}, \mathrm{NH} H), 4.12$ (td, $J=$ 8.6, $5.1 \mathrm{~Hz}, 1 \mathrm{H}, \mathrm{C}_{\alpha} \mathrm{H}$ Lysine), 2.99 (q, $J=6.6 \mathrm{~Hz}, 2 \mathrm{H}, \mathrm{C}_{\varepsilon} \mathrm{H}_{2}$ Lysine), 2.03 (t, $J=7.4 \mathrm{~Hz}, 2 \mathrm{H}$, $\mathrm{C}_{\alpha} \mathrm{H}_{2}$ Butanoyl), 1.75 (p, J=7.6 Hz, 2H, $\mathrm{C}_{\beta} \mathrm{H}_{2}$ Butanoyl), $1.67-1.55$ (m, 1H, 1xH C ${ }_{\beta} H \mathrm{H}$ Lysine), $1.53-1.39$ (m, 1H, $\mathrm{C}_{\beta} \mathrm{HH}$ Lysine), $1.40-1.31$ (m, 2H, $\mathrm{C}_{\delta} \mathrm{H}_{2}$ Lysine), $1.30-1.16\left(\mathrm{~m}, 2 \mathrm{H}, \mathrm{C}_{\mathrm{\gamma}} \mathrm{H}_{2}\right.$ Lysine). Signal of $\mathrm{C}_{\mathrm{\gamma}} \mathrm{H}_{2}$ Butanoyl overlaps with solvent peak of DMSO. ${ }^{13} \mathrm{C}-\mathrm{NMR}$ (DMSO-d $\mathrm{d}_{6}$ ) $\delta$ $=173.87(\mathrm{C}=\mathrm{O}), 171.47(\mathrm{C}=\mathrm{O}), 169.09(\mathrm{C}=\mathrm{O}), 141.60$ (C-1 lodophenyl), 136,96 (C-3,5 lodophenyl), 130.87 (C-2,6 lodophenyl), 91.25 (C-4 lodophenyl), 52.23 ( $\mathrm{C}_{\alpha}$ Lysine), 38.26 ( $\mathrm{C}_{\varepsilon}$ Lysine), 34.65 ( $\mathrm{C}_{\alpha}$ Butanoyl), 34.05 ( $\mathrm{C}_{\mathrm{Y}}$ Butanoyl), 31.70 ( $\mathrm{C}_{\beta}$ Lysine), 28.90 ( $\mathrm{C}_{\delta}$ Lysine), 26.77 $\left(\mathrm{C}_{\beta}\right.$ Butanoyl), $22.88\left(\mathrm{C}_{\mathrm{V}}\right.$ Lysine $), 22.54\left(\mathrm{CH}_{3}\right.$ Acetyl). MS $\left(E S I^{+}\right): \mathrm{m} / \mathrm{z}$ calculated for $\mathrm{C}_{18} \mathrm{H}_{27} \mathrm{IN}_{3} \mathrm{O}_{3}: 460.11[\mathrm{M}+\mathrm{H}]^{+}$, found: 459.91 


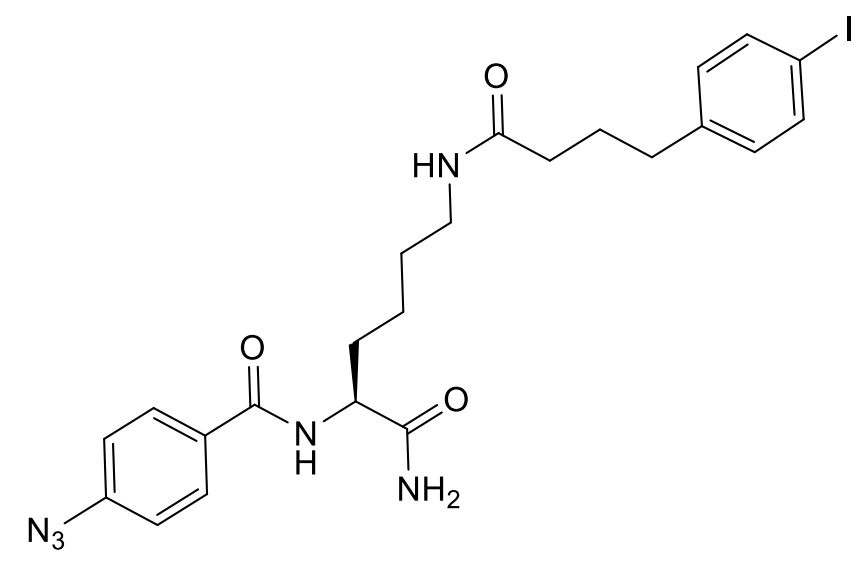

H_25-75_5iso_25min_1 $\mathrm{mL} \_42$ total $\mathrm{t}_{\mathrm{R}}=25.5 \mathrm{~min}$

Compound (S)-2b (40 mg, 36\% overall yield, slightly yellow solid) was synthesised starting from Fmoc-L-Lys(Boc)-OH according to GPs II, III, IV, VIIIb and XIII. ${ }^{1} \mathbf{H}-\mathbf{N M R}\left(\right.$ DMSO- $\left.d_{6}\right) \delta=$ $8.31\left(\mathrm{~d}, J=8.0 \mathrm{~Hz}, 1 \mathrm{H}, \mathrm{N}_{\mathrm{a}} \mathrm{H}\right.$ ), $7.94(\mathrm{~d}, J=8.6 \mathrm{~Hz}, 2 \mathrm{H}, \mathrm{H}-2,6$ Azidobenzoyl), 7.74 (t, $J=5.6 \mathrm{~Hz}$, $1 \mathrm{H}, \mathrm{N}_{\varepsilon} \mathrm{H}$ ), 7.60 (d, J = 8.2 Hz, 2H, H-3,5 lodophenyl), 7.37 (s, 1H, NHH), 7.18 (d, J = 8.6 Hz, $2 \mathrm{H}, \mathrm{H}-3,5$ Azidobenzoyl), $7.02-6.94(\mathrm{~m}, 3 \mathrm{H}, \mathrm{H}-2,6$ lodophenyl and $\mathrm{NHH}), 4.38-4.28(\mathrm{~m}, 1 \mathrm{H}$, $\mathrm{C}_{\alpha} \mathrm{H}$ Lysine), $3.07-2.94\left(\mathrm{~m}, 2 \mathrm{H}, \mathrm{C}_{\varepsilon} \mathrm{H}_{2}\right.$ Lysine), $2.47^{*}$ (t, $J=7.6 \mathrm{~Hz}, 2 \mathrm{H}, \mathrm{C}_{\gamma} \mathrm{H}_{2}$ Butanoyl), 2.01 (t, $J=7.4 \mathrm{~Hz}, 2 \mathrm{H}, \mathrm{C}_{\alpha} \mathrm{H}_{2}$ Butanoyl), 1.73 (tdt, $J=13.1,9.1,6.5 \mathrm{~Hz}, 4 \mathrm{H}, \mathrm{C}_{\beta} \mathrm{H}_{2}$ Lysine and $\mathrm{C}_{\beta} \mathrm{H}_{2}$ Butanoyl), $1.44-1.19\left(\mathrm{~m}, 4 \mathrm{H}, \mathrm{C}_{\delta} \mathrm{H}_{2}\right.$ Lysine and $\mathrm{C}_{\mathrm{\gamma}} \mathrm{H}_{2}$ Lysine). *Signal partly overlaps with DMSO solvent peak. ${ }^{13} \mathrm{C}-\mathrm{NMR}\left(\mathrm{DMSO}-d_{6}\right) \delta=173.90(\mathrm{C}=\mathrm{O}), 171.48(\mathrm{C}=\mathrm{O}), 165.32(\mathrm{C}=\mathrm{O})$, 142.25 (C-4 Azidobenzoyl), 141.56 (C-4 lodophenyl), 136.93 (C-3,5 lodophenyl), 130.83 (C2,6 lodophenyl), 129.42 (C-2,6 Azidobenzoyl), 118.71 (C-3,5 Azidobenzoyl), 91.23 (C-1 lodophenyl), 53.24 ( $\mathrm{C}_{\alpha}$ Lysine), 38.20 ( $\mathrm{C}_{\varepsilon}$ Lysine), 34.65 ( $\mathrm{C}_{\alpha}$ Butanoyl), $34.02\left(\mathrm{C}_{\mathrm{\gamma}}\right.$ Butanoyl), 31.26 ( $\mathrm{C}_{\beta}$ Lysine), 28.89 ( $\mathrm{C}_{\delta}$ Lysine), 26.75 ( $\mathrm{C}_{\beta}$ Butanoyl), $23.23\left(\mathrm{C}_{\mathrm{Y}}\right.$ Lysine). MS (ESI $\left.{ }^{+}\right): \mathrm{m} / \mathrm{z}$ calculated for $\mathrm{C}_{23} \mathrm{H}_{27} \mathrm{IN}_{6} \mathrm{NaO}_{3}: 585.11[\mathrm{M}+\mathrm{Na}]^{+}$, found: $584.72[\mathrm{M}+\mathrm{Na}]^{+}$ 


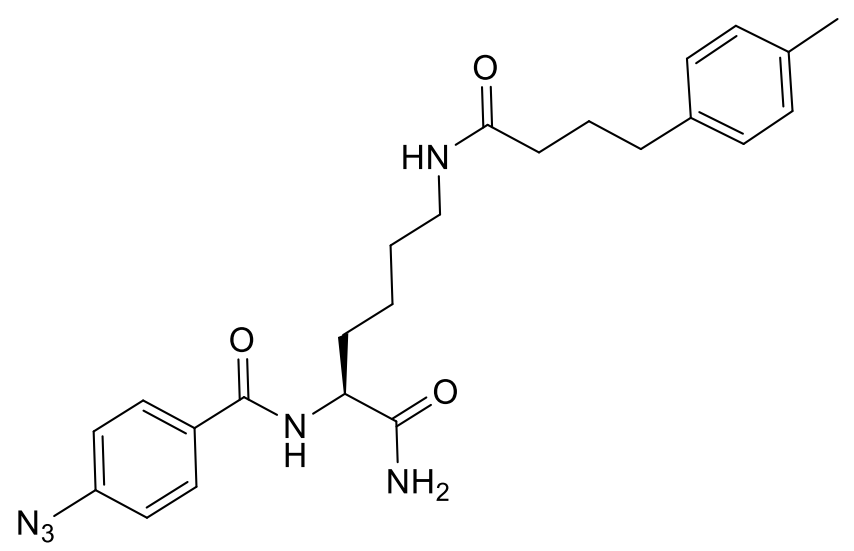

\section{H_25-75_5iso_25min_1 $\mathrm{mL}$ 42total $\mathrm{t}_{\mathrm{R}}=23.5 \mathrm{~min}$}

Compound (S)-2c (30 mg, 33\% overall yield, slightly yellow solid) was synthesised starting from Fmoc-L-Lys(Boc)-OH according to GPs II, III, IV, VIIIb and XIII. ${ }^{1}$ H-NMR (DMSO-d $\left.{ }_{6}\right) \delta=$ $8.32\left(\mathrm{~d}, J=8.0 \mathrm{~Hz}, 1 \mathrm{H}, \mathrm{N}_{\mathrm{a}} \mathrm{H}\right.$ ), $7.95(\mathrm{~d}, J=8.7 \mathrm{~Hz}, 2 \mathrm{H}, \mathrm{H}-2,6$ Azidobenzoyl), $7.73(\mathrm{t}, J=5.7 \mathrm{~Hz}$, $1 \mathrm{H}, \mathrm{N}_{\varepsilon} \mathrm{H}$ ), $7.37(\mathrm{~s}, 1 \mathrm{H}, \mathrm{NHH}), 7.18(\mathrm{~d}, J=8.6 \mathrm{~Hz}, 2 \mathrm{H}, \mathrm{H}-3,5$ Azidobenzoyl), 7.05 (q, J = $8.1 \mathrm{~Hz}$, $4 \mathrm{H}, \mathrm{H}-2,3,5,6$ Tolyl), 6.98 (s, 1H, NHH), $4.38-4.28$ (m, 1H, CaH Lysine), $3.06-2.95$ (m, 2H, $\mathrm{C}_{\varepsilon} \mathrm{H}_{2}$ Lysine), 2.46* (t, J = 7.6 Hz, 2H, $\mathrm{C}_{\mathrm{\gamma}} \mathrm{H}_{2}$ Butanoyl), 2.25 (s, 3H, $\mathrm{CH}_{3}$ ), 2.01 (t, $J=7.5 \mathrm{~Hz}$, $2 \mathrm{H}, \mathrm{C}_{\alpha} \mathrm{H}_{2}$ Butanoyl), $1.79-1.65\left(\mathrm{~m}, 4 \mathrm{H}, \mathrm{C}_{\beta} \mathrm{H}_{2}\right.$ Lysine and $\mathrm{C}_{\beta} \mathrm{H}_{2}$ Butanoyl), $1.44-1.22(\mathrm{~m}, 4 \mathrm{H}$, $\mathrm{C}_{\delta} \mathrm{H}_{2}$ Lysine and $\mathrm{C}_{\mathrm{\gamma}} \mathrm{H}_{2}$ Lysine). ${ }^{*}$ Signal partly overlaps with DMSO solvent peak. ${ }^{13} \mathrm{C}-\mathrm{NMR}$ $\left(\mathrm{DMSO}_{-} \mathrm{d}_{6}\right) \delta=173.90(\mathrm{C}=\mathrm{O}), 171.62(\mathrm{C}=\mathrm{O}), 165.32(\mathrm{C}=\mathrm{O}), 142.24$ (C-4 Azidobenzoyl), 138.60 (C-1/4 Tolyl), 134.52 (C-1/4 Tolyl), 130.78 (C-1 Azidobenzoyl), 129.42 (C-2,6 Azidobenzoyl), 128.80 (2xCH Tolyl), 128.15 (2xCH Tolyl), 118.71 (C-3,5 Azidobenzoyl), 53.24 ( $C_{\alpha}$ Lysine), 38.19 ( $\mathrm{C}_{\varepsilon}$ Lysine), 34.83 ( $\mathrm{C}_{\alpha}$ Butanoyl), 34.23 ( $\mathrm{C}_{\mathrm{\gamma}}$ Butanoyl), 31.25 ( $\mathrm{C}_{\beta}$ Lysine), 28.90 ( $\mathrm{C}_{\mathrm{y}}$ Lysine), $27.13\left(\mathrm{C}_{\beta}\right.$ Butanoyl), $23.22\left(\mathrm{C}_{\mathrm{y}}\right.$ Lysine $), 20.58\left(\mathrm{CH}_{3}\right) . \mathrm{MS}\left(\mathrm{ESI}^{+}\right): \mathrm{m} / \mathrm{z}$ calculated for $\mathrm{C}_{24} \mathrm{H}_{30} \mathrm{~N}_{6} \mathrm{NaO}_{3}: 473.23[\mathrm{M}+\mathrm{Na}]^{+}$, found: $472.83[\mathrm{M}+\mathrm{Na}]^{+}$ 


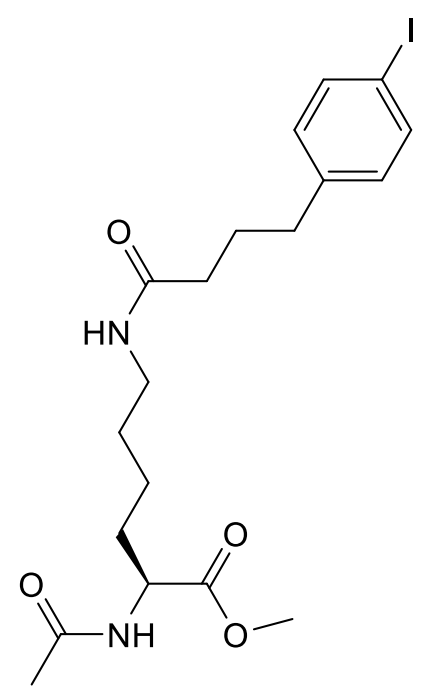

H_25-75_5iso_25min_1 $\mathrm{mL} \_42$ total $\mathrm{t}_{\mathrm{R}}=22.6 \mathrm{~min}$

Compound (S)-3a (72 mg, 39\% overall yield, clear oil) was synthesised starting from Boc-LLys-OMe according to GPs XIII, XIV, XIII. ${ }^{1} \mathrm{H}-\mathbf{N M R}\left(\mathrm{CDCl}_{3}\right) \delta=7.59$ (d, J=8.3 Hz, 2H, H-3,5 lodophenyl), 6.92 (d, J = 8.0 Hz, 2H, H-2,6 lodophenyl), 6.36 (d, $J=7.8 \mathrm{~Hz}, 1 \mathrm{H}, \mathrm{N}_{\mathrm{a}} \mathrm{H}$ ), 5.84 (t, $J=4.9 \mathrm{~Hz}, 1 \mathrm{H}, \mathrm{N}_{\varepsilon} \mathrm{H}$ ), 4.57 (td, $J=8.1,4.6 \mathrm{~Hz}, 1 \mathrm{H}, \mathrm{C}_{\alpha} \mathrm{H}$ Lysine), 3.74 (s, 3H, $\mathrm{CH}_{3}$ Methyl), 3.23 (dd, $J=6.6,5.1 \mathrm{~Hz}, 2 \mathrm{H}, \mathrm{C}_{\varepsilon} \mathrm{H}_{2}$ Lysine), 2.59 (t, $J=7.5 \mathrm{~Hz}, 2 \mathrm{H}, \mathrm{C}_{\mathrm{\gamma}} \mathrm{H}_{2}$ Butanoyl), $2.26-2.15$ (m, $1 \mathrm{H}, \mathrm{C}_{\alpha} \mathrm{H}_{2}$ Butanoyl), 2.03 (s, 3H, $\mathrm{CH}_{3}$ Acetyl), 1.94 (q, J = 8.0 Hz, 2H, $\mathrm{C}_{\beta} \mathrm{H}_{2}$ Butanoyl), 1.89 $1.79\left(\mathrm{~m}, 1 \mathrm{H}, \mathrm{C}_{\beta} H \mathrm{H}\right.$ Lysine), $1.74-1.64\left(\mathrm{~m}, 1 \mathrm{H}, \mathrm{C}_{\beta} \mathrm{H} H\right.$ Lysine $), 1.60-1.45\left(\mathrm{~m}, 2 \mathrm{H}, \mathrm{C}_{\delta} \mathrm{H}_{2}\right.$ Lysine), $1.41-1.29\left(\mathrm{~m}, 2 \mathrm{H}, \mathrm{C}_{\gamma} \mathrm{H}_{2}\right.$ Lysine). ${ }^{13} \mathrm{C}-\mathrm{NMR}\left(\mathrm{CDCl}_{3}\right) \delta=173.87(\mathrm{C}=\mathrm{O}), 172.85(\mathrm{C}=\mathrm{O})$, 171.07 (C=O), 141.09 (C-1 lodophenyl), 137.61 (C-3,5 lodophenyl), 130.72 (C-2,6 lodophenyl), 91.22 (C-4 lodophenyl), $52.66\left(\mathrm{CH}_{3}-\mathrm{O}\right), 52.01$ ( $\mathrm{C}_{\alpha}$ Lysine), 39.10 ( $\mathrm{C}_{\varepsilon}$ Lysine), 35.73 ( $\mathrm{C}_{\alpha}$ Butanoyl), 34.81 ( $\mathrm{C}_{\mathrm{\gamma}}$ Butanoyl), 32.11 ( $\mathrm{C}_{\beta}$ Lysine), 28.69 ( $\mathrm{C}_{\delta}$ Lysine), 27.10 ( $\mathrm{C}_{\beta}$ Butanoyl), 23.1 (Acetyl), 22.34 ( $\mathrm{C}_{\mathrm{y}}$ Lysine); $\mathrm{MS}\left(\mathrm{ESI}{ }^{+}\right)$: $\mathrm{m} / \mathrm{z}$ calculated for $\mathrm{C}_{19} \mathrm{H}_{28} \mathrm{IN}_{2} \mathrm{O}_{4}: 475.11$ $[\mathrm{M}+\mathrm{H}]^{+}$, found: 474.92 


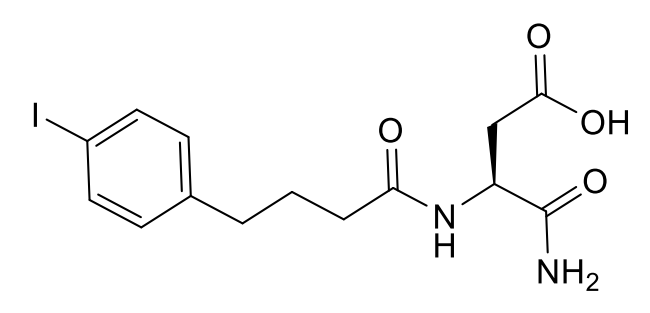

H_25-75_5iso_25min_1 $\mathrm{mL} \_42$ total $\mathrm{t}_{\mathrm{R}}=16.7 \mathrm{~min}$

Compound (S)-4a (14 mg, 35\% overall yield, white solid) was synthesised starting from FmocL-Asp(tBu)-OH according to GPs II, III, V and VIIIb. ${ }^{1} \mathrm{H}-\mathrm{NMR}$ (DMSO- $\left.d_{6}\right) \delta=8.01$ (d, $J=8.1$ $\mathrm{Hz}, 1 \mathrm{H}, \mathrm{N}_{\mathrm{a}} \mathrm{H}$ ), 7.62 (d, J = 8.2 Hz, 1H, H-3,5 lodophenyl), 7.21 (s, 1H, NHH), $7.05-6.99$ (m, $3 \mathrm{H}, \mathrm{NHH}$ and $\mathrm{H}-2,6$ lodophenyl), 4.51 (td, $J=8.1,5.6 \mathrm{~Hz}, 1 \mathrm{H}, \mathrm{C}_{\mathrm{a}} \mathrm{H}$ Asp), 2.64 (dd, $J=16.3$, $5.6 \mathrm{~Hz}, 1 \mathrm{H}, \mathrm{C}_{\beta} H \mathrm{H}$ Asp), 2.44 (dd, $J=16.3,8.2 \mathrm{~Hz}, 1 \mathrm{H}, \mathrm{C}_{\beta} \mathrm{H} H$ Asp), 2.10 (td, $J=7.2,2.2 \mathrm{~Hz}$, $2 \mathrm{H}, \mathrm{C}_{\alpha} \mathrm{H}_{2}$ Butanoyl), 1.75 ( $\mathrm{p}, \mathrm{J}=7.5 \mathrm{~Hz}, 2 \mathrm{H}, \mathrm{C}_{\beta} \mathrm{H}_{2}$ Butanoyl). Signal of $\mathrm{C}_{\mathrm{\gamma}} \mathrm{H}_{2}$ Butanoyl overlaps with DMSO solvent peak. ${ }^{13} \mathrm{C}$-NMR (DMSO- $\left.d_{6}\right) \delta=172.80(\mathrm{C}=\mathrm{O}), 171.96(\mathrm{C}=\mathrm{O}), 171.91$ $(\mathrm{C}=\mathrm{O}), 141.73$ (C-1 lodophenyl), 137.02 (C-3,5 lodophenyl), 130.99 (C-2,6 lodophenyl), 91.28 (C-4 lodophenyl), 49.29 ( $\mathrm{C}_{\alpha}$ Asp), 36.27 ( $\mathrm{C}_{\beta}$ Asp), 34.53 ( $\mathrm{C}_{\alpha}$ Butanoyl), 33.95 ( $\mathrm{C}_{\mathrm{\gamma}}$ Butanoyl), $26.73\left(\mathrm{C}_{\beta}\right.$ Butanoyl). MS $\left(\mathrm{ESI}^{+}\right): \mathrm{m} / \mathrm{z}$ calculated for $\mathrm{C}_{14} \mathrm{H}_{18} \mathrm{IN}_{2} \mathrm{O}_{4}: 405.03[\mathrm{M}+\mathrm{H}]^{+}$, found: 404.89 . 


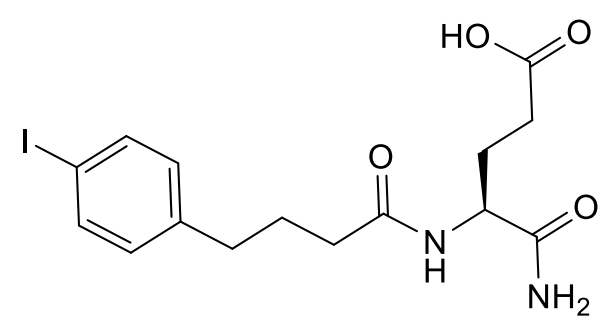

H_25-75_5iso_25min_1 $\mathrm{mL} \_42$ total $\mathrm{t}_{\mathrm{R}}=17.0 \mathrm{~min}$

Compound (S)-4b (12 mg, 29\% overall yield, white solid) was synthesised starting from FmocL-Glu(tBu)-OH according to GPs II, III, V and VIIIb. ${ }^{1} \mathbf{H}-N M R\left(D M S O-d_{6}\right) \delta=7.86(d, J=8.1$ $\mathrm{Hz}, 1 \mathrm{H}, \mathrm{N}_{\mathrm{a}} \mathrm{H}$ ), 7.62 (d, J = 8.2 Hz, 1H, H-3,5 lodophenyl), 7.29 (s, $\left.1 \mathrm{H}, \mathrm{NHH}\right), 7.04-6.97$ (m, $3 \mathrm{H}, \mathrm{NHH}$ and $\mathrm{H}-2,6$ lodophenyl), 4.18 (td, $J=8.5,5.1 \mathrm{~Hz}, 1 \mathrm{H}, \mathrm{C}_{\mathrm{a}} \mathrm{H} \mathrm{Glu}$ ), 2.21 (dd, $J=8.4,7.3$ $\mathrm{Hz}, 2 \mathrm{H}, \mathrm{C}_{\mathrm{\gamma}} \mathrm{H}_{2}$ Glutamat), 2.13 (t, $J=7.4 \mathrm{~Hz}, 2 \mathrm{H}, \mathrm{C}_{\alpha} \mathrm{H}_{2}$ Butanoyl), $1.94-1.84\left(\mathrm{~m}, 1 \mathrm{H}, \mathrm{C}_{\beta} \mathrm{HH}\right.$ Glu), $1.80-1.64\left(\mathrm{~m}, 3 \mathrm{H}, \mathrm{C}_{\beta} \mathrm{HH}\right.$ Glu and $\mathrm{C}_{\beta} \mathrm{H}_{2}$ Butanoyl). Signal of $\mathrm{C}_{\mathrm{\gamma}} \mathrm{H}_{2}$ Butanoyl overlapping with DMSO solvent peak. ${ }^{13} \mathrm{C}$-NMR (DMSO- $\left.\mathrm{d}_{6}\right) \delta=173.90(\mathrm{C}=\mathrm{O}), 173.38(\mathrm{C}=\mathrm{O}), 171.87$ (C=O), 141.66 (C-1 lodophenyl), 136.96 (C-3,5 lodophenyl), 130.89 (C-2,6 lodophenyl), 91.25 (C-4 lodophenyl), 51.60 ( $\left.\mathrm{C}_{\alpha} \mathrm{Glu}\right), 34.44$ ( $\mathrm{C}_{\alpha}$ Butanoyl), 34.00 ( $\mathrm{C}_{\mathrm{Y}}$ Butanoyl), $30.27\left(\mathrm{C}_{\mathrm{y}} \mathrm{Glu}\right)$, $27.25\left(\mathrm{C}_{\beta} \mathrm{Glu}\right), 26.72\left(\mathrm{C}_{\beta}\right.$ Butanoyl). MS $\left(\mathrm{ESI}^{+}\right): \mathrm{m} / \mathrm{z}$ calculated for $\mathrm{C}_{15} \mathrm{H}_{20} \mathrm{IN}_{2} \mathrm{O}_{4}: 419.05[\mathrm{M}+\mathrm{H}]^{+}$, found: 418.90 


\section{Intermediates}

$N^{\varepsilon}$-(4-(4-lodophenyl)butanoyl)-L-lysine*TFA ((S)-6)

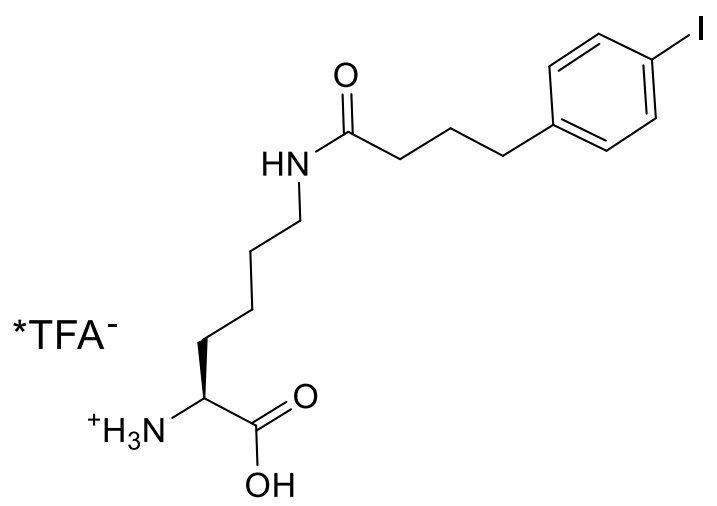

Compound (S)-6 (550 mg, quantitative, white solid) was synthesised using (S)-9 according to GP XIV. ${ }^{1} \mathrm{H}-\mathrm{NMR}\left(\mathrm{DMSO}-d_{6}\right) \delta=13.86(\mathrm{br} \mathrm{s}, 1 \mathrm{H}, \mathrm{COOH}), 8.21\left(\mathrm{~s}, 3 \mathrm{H}, \mathrm{NH}_{3}{ }^{+}\right), 7.76(\mathrm{t}, J=5.6$ $\mathrm{Hz}, 1 \mathrm{H}, \mathrm{N}_{\varepsilon} \mathrm{H}$ ), 7.62 (d, J = 8.4 Hz, 2H, H-3,5 lodophenyl), 7.01 (d, J = 8.4 Hz, 2H, H-2,6 lodophenyl), 3.87 (s, 1H, $\mathrm{C}_{\alpha} \mathrm{H}$ Lysine), 3.01 (q, $J=6.3 \mathrm{~Hz}, 2 \mathrm{H}, \mathrm{C}_{\varepsilon} \mathrm{H}_{2}$ Lysine), 2.04 (t, $J=7.5$ $\mathrm{Hz}, 2 \mathrm{H}, \mathrm{C}_{\alpha} \mathrm{H}$ Butanoyl), 1.75 (p, J=7.9 Hz, $4 \mathrm{H}, \mathrm{C}_{\beta} \mathrm{H}_{2}$ Butanoyl and $\mathrm{C}_{\beta} \mathrm{H}_{2}$ Lysine), $1.43-1.26$ ( $m, 4 \mathrm{H}, \mathrm{C}_{\delta} \mathrm{H}_{2}$ and $\mathrm{C}_{\mathrm{\gamma}} \mathrm{H}_{2}$ Lysine). Signal of $\mathrm{C}_{\mathrm{\gamma}} \mathrm{H}_{2}$ Butanoyl overlaps with DMO solvent signal. MS $\left(E S I^{+}\right): m / z$ calculated for $\mathrm{C}_{15} \mathrm{H}_{20} \mathrm{IN}_{2} \mathrm{O}_{4}: 419.08[\mathrm{M}+\mathrm{H}]^{+}$, found: 418.983

The ${ }^{13} \mathrm{C}-\mathrm{NMR}$ spectrum has not been recorded. 


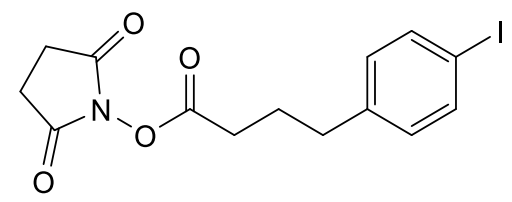

4-(4-lodophenyl)butanoic acid (504 mg, $1.74 \mathrm{mmol}, 1$ eq.) and NHS (221 mg, $1.91 \mathrm{mmol}, 1.1$ eq.) were dissolved in 1,4-dioxane $(7 \mathrm{~mL})$. To this mixture, DIC $(0.46 \mathrm{ml}, 1.66 \mathrm{mmol}, 0.95$ eq. was added. The reaction was stirred at for $15 \mathrm{~h}$. The reaction progress was followed by TLC (DCM). 1,4-Dioxane was removed under reduced pressure. This concentrated mixture was dissolved in $\mathrm{CH}_{2} \mathrm{Cl}_{2}$, filtered and purified by column chromatography $\left(\mathrm{CH}_{2} \mathrm{Cl}_{2} /\right.$ diethyl ether 1:1, $\left.\mathrm{R}_{\mathrm{f}}=0.5\right)$ to yield $608 \mathrm{mg}(90 \%)$ of $7 \mathrm{a}$ as white solid. ${ }^{1} \mathrm{H}-\mathrm{NMR}\left(\mathrm{CDCl}_{3}\right) \delta=7.62(\mathrm{~d}, J=8.4 \mathrm{~Hz}$, $2 \mathrm{H}, \mathrm{H}-3,5$ lodophenyl), 6.96 (d, $J=8.5 \mathrm{~Hz}, 2 \mathrm{H}, \mathrm{H}-2,6$ lodophenyl), 2.85 (s, 4H, 2xCH $\mathrm{NHS}$ ), $2.73-2.64$ (m, 2H, $\mathrm{C}_{\mathrm{\gamma}} \mathrm{H}_{2}$ Butanoyl) 2.60 (t, $J=7.3 \mathrm{~Hz}, 2 \mathrm{H}, \mathrm{C}_{\alpha} \mathrm{H}_{2}$ Butanoyl) 2.04 (quint., $J=7.4$ $\mathrm{Hz}, 2 \mathrm{H}, \mathrm{C}_{\beta} \mathrm{H}_{2}$ Butanoyl). ${ }^{13} \mathrm{C}-\mathrm{NMR}\left(\mathrm{CDCl}_{3}\right) \delta=169.22$ (2xCO NHS), 168.44 (COO), 140.39 (C1 lodophenyl), 137.74 (C-3,5 lodophenyl), 130.79 (C-2,6 lodophenyl), 91.51 (C-4 lodophenyl), 34.15 ( $\mathrm{C}_{\mathrm{Y}}$ Butanoyl), $30.22\left(\mathrm{C}_{\alpha}\right), 26.17$ ( $\mathrm{C}_{\beta}$ Butanoyl, $25.75\left(2 \mathrm{xCH}_{2} \mathrm{NHS}\right)$.

${ }^{1} \mathrm{H}$ - and ${ }^{13} \mathrm{C}-N M R$ data are in agreement to those reported in literature. ${ }^{17}$ 


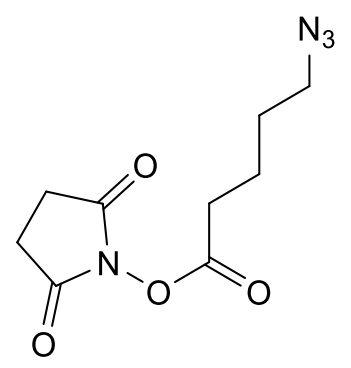

5-azidopentynoic acid (150 mg, $1.05 \mathrm{mmol}, 1$ eq.) and NHS (121 mg, $1.05 \mathrm{mmol}, 1$ eq.) were dissolved in 1,4-dioxane $(4 \mathrm{~mL})$. To this mixture, DIC $(0.46 \mathrm{ml}, 0.95 \mathrm{mmol}, 0.95$ eq.) was added. The reaction was stirred at for $22 \mathrm{~h}$. The reaction progress was followed by TLC $\left(\mathrm{CH}_{2} \mathrm{Cl}_{2}\right)$. The solution was adsorbed to silica gel and purified by column chromatography $\left(\mathrm{CH}_{2} \mathrm{Cl}_{2} /\right.$ diethyl ether 9:1, $\left.\mathrm{R}_{\mathrm{f}}=0.6\right)$ to yield $187 \mathrm{mg}(70 \%)$ of $7 \mathrm{~b}$ as a yellowish oil (wax at $4^{\circ} \mathrm{C}$ ). ${ }^{1} \mathrm{H}$ NMR $\left(\mathrm{CDCl}_{3}\right) \delta=3.34\left(\mathrm{~m}, 2 \mathrm{H}, \mathrm{C}_{\delta} \mathrm{H}_{2}-\mathrm{N}_{3}\right), 2.84\left(\mathrm{~s}, 4 \mathrm{H}, 2 \mathrm{CH}_{2}\right.$ of $\left.\mathrm{NHS}\right), 2.66\left(\mathrm{~m}, 2 \mathrm{H}, \mathrm{C}_{a} \mathrm{H}_{2}\right)$, $1.92-1.79\left(\mathrm{~m}, 2 \mathrm{H}, \mathrm{C}_{\beta} \mathrm{H}_{2}\right), 1.78-1.60\left(\mathrm{~m}, 2 \mathrm{H}, \mathrm{C}_{\mathrm{\gamma}} \mathrm{H}_{2}\right) \cdot{ }^{13} \mathrm{C}-\mathrm{NMR}\left(\mathrm{CDCl}_{3}\right) \delta=169.20(2 \times \mathrm{C}=\mathrm{O})$, $168.31(\mathrm{C}=\mathrm{O}), 50.97\left(\mathrm{C}_{\delta}\right), 30.58\left(\mathrm{C}_{\alpha}\right), 28.02\left(\mathrm{C}_{\beta}\right), 25.73\left(2 \mathrm{xCH}_{2}\right.$ of pyrrolidine $), 21.99\left(\mathrm{C}_{\mathrm{\gamma}}\right)$.

${ }^{1} \mathrm{H}$ - and ${ }^{13} \mathrm{C}-\mathrm{NMR}$ data are in agreement to those reported in literature. ${ }^{18}$ 


\section{4-Azidobenzoic acid ${ }^{19}$}<smiles>Nc1ccc(C(=O)O)cc1</smiles>

To a three-necked flask, 4-aminobenzoic acid (5 g, $40 \mathrm{mmol}, 1$ eq.), ethanol ( $400 \mathrm{~mL}$ ), and conc. hydrochloric acid $(200 \mathrm{~mL})$ were added, and the mixture was stirred at $0^{\circ} \mathrm{C}$ for $30 \mathrm{~min}$. Subsequently, an aqueous solution $(100 \mathrm{~mL})$ of sodium nitrite $(4.15 \mathrm{~g}, 60 \mathrm{mmol}, 1.5 \mathrm{eq}$.) was added dropwise. The reaction mixture was stirred at 0 for $1 \mathrm{~h}$. This diazonium solution was kept at $0^{\circ} \mathrm{C}$ for the subsequent reaction. Sodium azide $(13 \mathrm{~g}, 201 \mathrm{mmol}, 5 \mathrm{eq}$.), ethanol $(100 \mathrm{~mL})$, and water $(100 \mathrm{~mL})$ were added into a three-necked flask and the mixture was stirred at $0^{\circ} \mathrm{C}$. To this solution, the prepared diazonium solution was added dropwise and the reaction mixture was stirred at room temperature overnight. Ethanol was removed in vacuo and a solid rapidly was formed. The solid was filtrated and washed with $1 \mathrm{~N} \mathrm{HCl}$. The product obtained was dried under reduced pressure and used for the subsequent reaction without purification. 4Azidobenzoic acid was obtained as yellow solid in quantitative yield (6.5 g). ${ }^{1} \mathrm{H}-\mathrm{NMR}$ (DMSO$\left.\mathrm{d}_{6}\right) \delta=7.97(\mathrm{~d}, J=8.8 \mathrm{~Hz}, 2 \mathrm{H}, \mathrm{H}-2,6), 7.22(\mathrm{~d}, J=8.4 \mathrm{~Hz}, 2 \mathrm{H}, \mathrm{H}-3,5) .{ }^{13} \mathrm{C}-\mathrm{NMR}$ (DMSO-d 6 ) $\delta$ $=166.54$ (COO), 143.95 (C-4), 131.21 (C-2,6), 127.29 (C1), 119.18 (C-3,5).

NMR data are in agreement with those reported in the literature. ${ }^{19}$ 
<smiles>Nc1ccc(C(=O)ON2C(=O)CCC2=O)cc1</smiles>

4-azidobenzoic acid (505 mg, $3.1 \mathrm{mmol}, 1$ eq.) and NHS (392 mg, $3.41 \mathrm{mmol}, 1.1$ eq.) were dissolved in 1,4-dioxane ( $12 \mathrm{~mL})$ cooled to $0^{\circ} \mathrm{C}$. To this mixture, DIC $(0.46 \mathrm{ml}, 3.13 \mathrm{mmol}$, 1eq.) was added. The reaction was stirred in the dark under $\mathrm{N}_{2}$ at $0^{\circ} \mathrm{C}$ for approximately $1 \mathrm{~h}$ and then at $\mathrm{rt}$ for $15 \mathrm{~h}$. 1,4-Dioxane was removed in vacuo. This concentrated mixture was dissolved in $\mathrm{CH}_{2} \mathrm{Cl}_{2}$, filtered and purified by coloumn chromatography $\left(\mathrm{CH}_{2} \mathrm{Cl}_{2}\right)$ to yield $790 \mathrm{mg}$ $(98 \%)$ of $7 \mathrm{c}$ as yellow solid. ${ }^{1} \mathrm{H}-\mathrm{NMR}\left(\mathrm{DMSO}_{-} \mathrm{d}_{6}\right) \delta=8.11(\mathrm{~d}, \mathrm{~J}=9.0 \mathrm{~Hz}, 2 \mathrm{H}, \mathrm{H}-2,6$ Azidobenzoyl), 7.36 (d, J = 9.0 Hz, 2H, H-3,5 Azidobenzoyl), 2.89 (s, 4H, 2xCH $\mathrm{CHS}{ }^{13} \mathrm{C}$ NMR (DMSO-d $)$ ) $\delta=170.30$ (2xCO NHS), 161.06 (COO), 146.81 (C-4), 132.00 (C-2,6), 120.48 (C-1), 120.12 (C-3,5), $25.52\left(2 \times \mathrm{CH}_{2} \mathrm{NHS}\right)$.

NMR data are in agreement with those reported in the literature. ${ }^{20}$ 


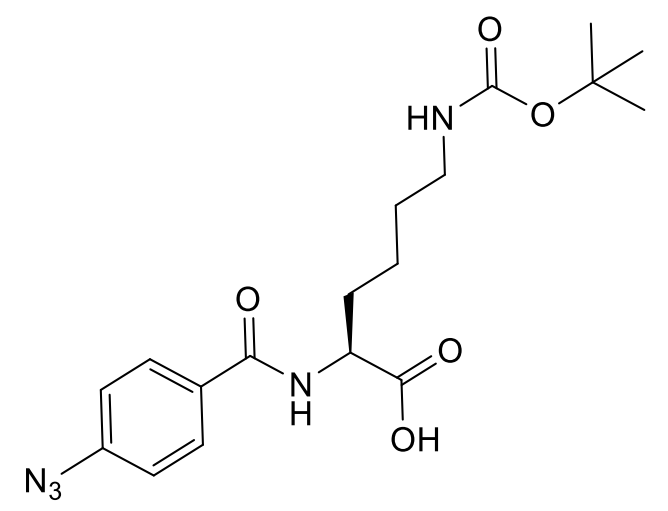

H_25-75_5iso_25min_1 $\mathrm{mL} \_42$ total $t_{R}=22.5 \mathrm{~min}$

Compound (S)-8 (955 mg, 79\%, pale white solid) was synthesised starting from L-Lys(Boc)$\mathrm{OH}$ according to GP XII. ${ }^{1} \mathrm{H}-\mathrm{NMR}$ (DMSO-d $)_{6} \delta=12.50$ (br s, $1 \mathrm{H}, \mathrm{COOH}$ ), 8.57 (d, $J=7.6 \mathrm{~Hz}$, $1 \mathrm{H}, \mathrm{N}_{\mathrm{a}} \mathrm{H}$ ), 7.94 (d, $J=8.7 \mathrm{~Hz}, 2 \mathrm{H}, \mathrm{H}-2,6$ Azidobenzoyl), 7.21 (d, $J=8.6 \mathrm{~Hz}, 2 \mathrm{H}, \mathrm{H}-3,5$ Azidobenzoyl), $6.76\left(\mathrm{t}, J=5.7 \mathrm{~Hz}, 1 \mathrm{H}, \mathrm{N}_{\varepsilon} \mathrm{H}\right), 4.32\left(\mathrm{q}, J=7.6 \mathrm{~Hz}, 1 \mathrm{H}, \mathrm{C}_{\mathrm{a}} \mathrm{H}\right), 2.93-2.86(\mathrm{~m}, 2 \mathrm{H}$, $\mathrm{C}_{\varepsilon} \mathrm{H}_{2}$ ), 1.77 (dt, $J=14.2,7.8 \mathrm{~Hz}, 2 \mathrm{H}, \mathrm{C}_{\beta} \mathrm{H}_{2}$ ), 1.35 (br s, 13H, 3xCH $\mathrm{CH}_{3}$ Boc, $\mathrm{C}_{\delta} \mathrm{H}_{2}$ and $\mathrm{C}_{\gamma} \mathrm{H}_{2}$ Lysine). The ${ }^{13} \mathrm{C}-\mathrm{NMR}$ spectrum has not been recorded. 


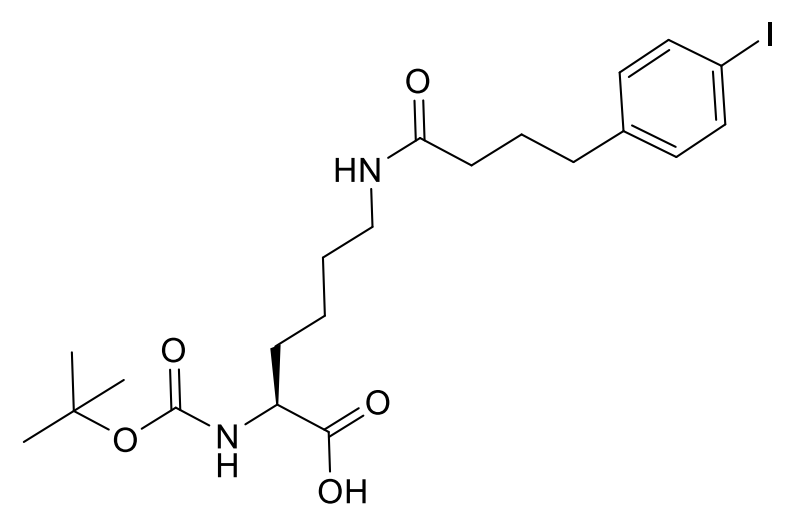

H_25-75_5iso_25min_1 $\mathrm{mL}$ 42total $\mathrm{t}_{\mathrm{R}}=26.7 \mathrm{~min}$

Compound (S)-9 (510 mg, 78\%, clear colorless oil) was synthesised starting from Boc-L-Lys$\mathrm{OH}$ according to GP XI. ${ }^{1} \mathrm{H}-\mathrm{NMR}$ (DMSO- $\mathrm{d}_{6}$ ) $\delta=12.38$ (br s, $\left.1 \mathrm{H}, \mathrm{COOH}\right), 7.74(\mathrm{t}, J=5.6 \mathrm{~Hz}$, $1 \mathrm{H}, \mathrm{N}_{\mathrm{a}} \mathrm{H}$ ), $7.62(\mathrm{~d}, J=8.3 \mathrm{~Hz}, 2 \mathrm{H}, \mathrm{H}-3,5$ lodophenyl), 7.01 (d, $J=8.1 \mathrm{~Hz}, 2 \mathrm{H}, \mathrm{H}-2,6$ lodophenyl), $3.86-3.76\left(\mathrm{~m}, 1 \mathrm{H}, \mathrm{C}_{a} \mathrm{H}\right.$ Lysine), $3.03-2.94$ (m, 2H, $\mathrm{C}_{\varepsilon} \mathrm{H}_{2}$ Lysine), 2.03 (t, $J=7.4 \mathrm{~Hz}, 2 \mathrm{H}, \mathrm{C}_{a} \mathrm{H}$ Butanoyl), 1.75 (p, J = 7.5 Hz, 2H, $\mathrm{C}_{\beta} \mathrm{H}_{2}$ Butanoyl), $1.65-1.49$ (m, 2H, $\mathrm{C}_{\beta} \mathrm{H}_{2}$ Lysine), 1.37 (br $\mathrm{s}, 13 \mathrm{H}, 3 \times \mathrm{CH}_{3} \mathrm{Boc}, \mathrm{C}_{\delta} \mathrm{H}_{2}$ and $\mathrm{C}_{\gamma} \mathrm{H}_{2}$ Lysine). The signal of $\mathrm{C}_{\gamma} \mathrm{H}_{2}$ Butanoyl overlaps with DMSO solvent signal.

The ${ }^{13} \mathrm{C}-\mathrm{NMR}$ spectrum has not been recorded. 


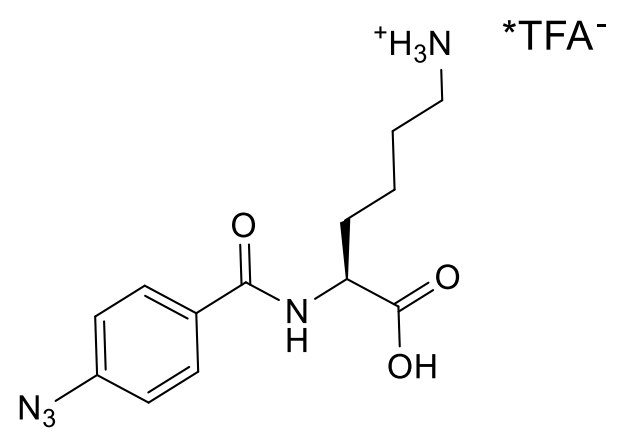

Compound (S)-10 (1010 mg, 79\%, orange oil) was synthesised using (S)-8 according to GP XIV. ${ }^{1} \mathrm{H}-\mathrm{NMR}$ (DMSO-d $\left.\mathrm{d}_{6}\right) \delta=12.65$ (br s, $1 \mathrm{H}, \mathrm{COOH}$ ), 8.61 (d, $J=7.8 \mathrm{~Hz}, 1 \mathrm{H}, \mathrm{N}_{\mathrm{a}} \mathrm{H}$ ), 7.94 (d, $J=8.7 \mathrm{~Hz}, 2 \mathrm{H}, \mathrm{H}-2,6$ Azidobenzoyl), $7.71\left(\mathrm{~s}, 3 \mathrm{H}, \mathrm{NH}_{3}{ }^{+}\right), 7.22(\mathrm{~d}, J=8.6 \mathrm{~Hz}, 2 \mathrm{H}, \mathrm{H}-3,5$ Azidobenzoyl), $4.46-4.29\left(\mathrm{~m}, 1 \mathrm{H}, \mathrm{C}_{\mathrm{a}} \mathrm{H}\right), 2.78\left(\mathrm{q}, J=6.2 \mathrm{~Hz}, 2 \mathrm{H}, \mathrm{C}_{\varepsilon} \mathrm{H}_{2}\right), 1.89-1.71(\mathrm{~m}, 2 \mathrm{H}$, $\left.\mathrm{C}_{\beta} \mathrm{H}_{2}\right), 1.63-1.51\left(\mathrm{~m}, 2 \mathrm{H}, \mathrm{C}_{\delta} \mathrm{H}_{2}\right), 1.51-1.32\left(\mathrm{~m}, 2 \mathrm{H}, \mathrm{C}_{\gamma} \mathrm{H}_{2}\right)$.

The ${ }^{13} \mathrm{C}-\mathrm{NMR}$ spectrum has not been recorded. 


\section{TATE derivatives}

\section{NODAGA-TATE}

NODAGA-TATE (53 mg, 21\%, white solid) was synthesized starting from Fmoc-Thr(tBu)-Wang resin according to GPs III and V (repeptitive cycles), followed by GP VIIIc and XV.

HPLC-Spectrum

H_05-55_5iso_25min_1mL_42total

mAU

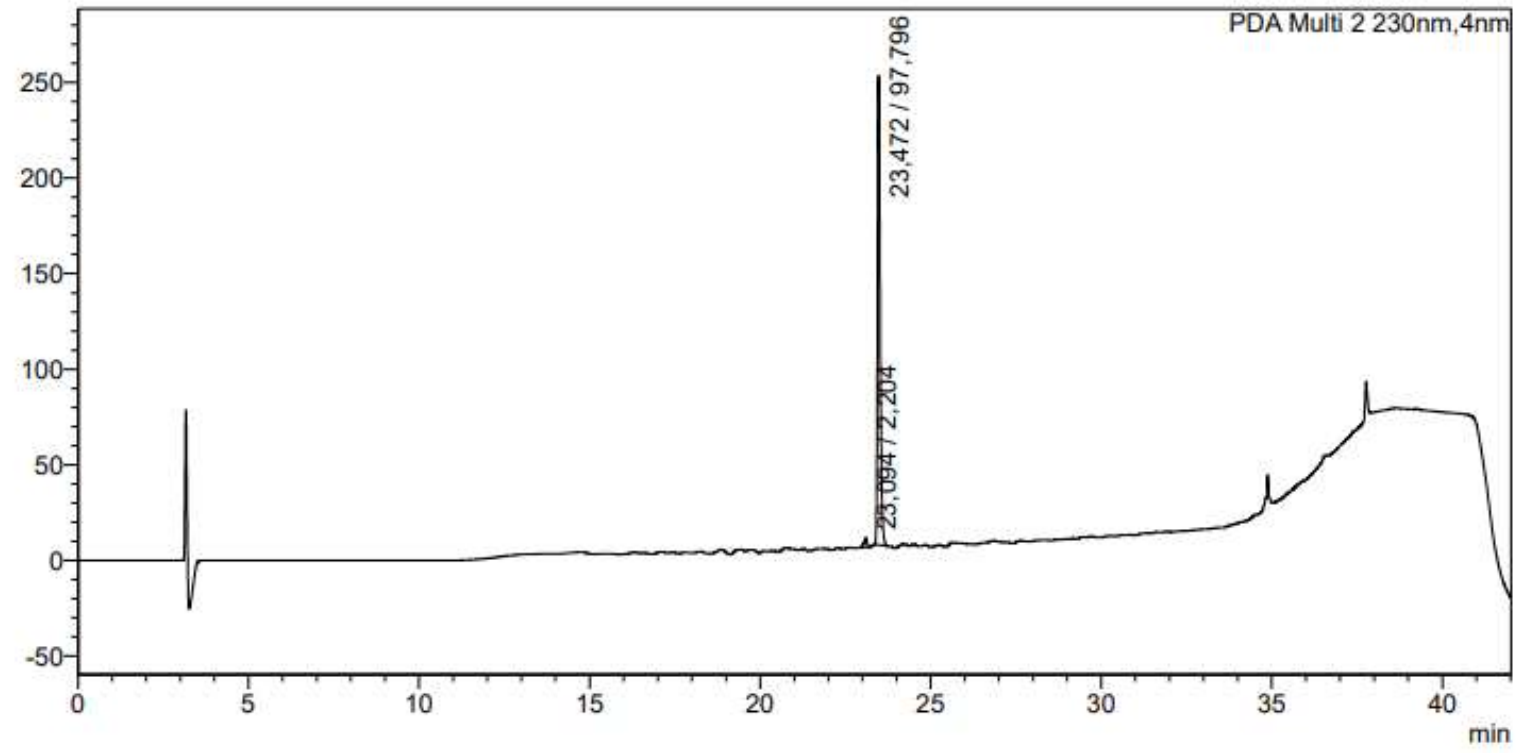

\begin{tabular}{rrrrrrr}
\multicolumn{2}{l}{ PDA Ch2 230nm } & \multicolumn{1}{l}{ Arrea } & Height & Conc. & Unit & \multicolumn{1}{c}{ Area\% } \\
Peak\# & Ret. Time & \multicolumn{1}{c}{ Area } \\
1 & 23,094 & 29296 & 4911 & 0,000 & 2,204 \\
2 & 23,472 & 1300112 & 244057 & 0,000 & 97,796 \\
\hline Total & & 1329407 & 248968 & & & 100,000
\end{tabular}




\section{HRMS spectrum}

Sections of recorded mass spectra around the signal of $[\mathrm{M}+2 \mathrm{H}]^{2+}$ are shown on the left side versus the predicted $[\mathrm{M}+2 \mathrm{H}]^{2+}$ signal on the right side. Prediction of mass spectra was performed using the software MestreNova (version 14.2.1-27684).
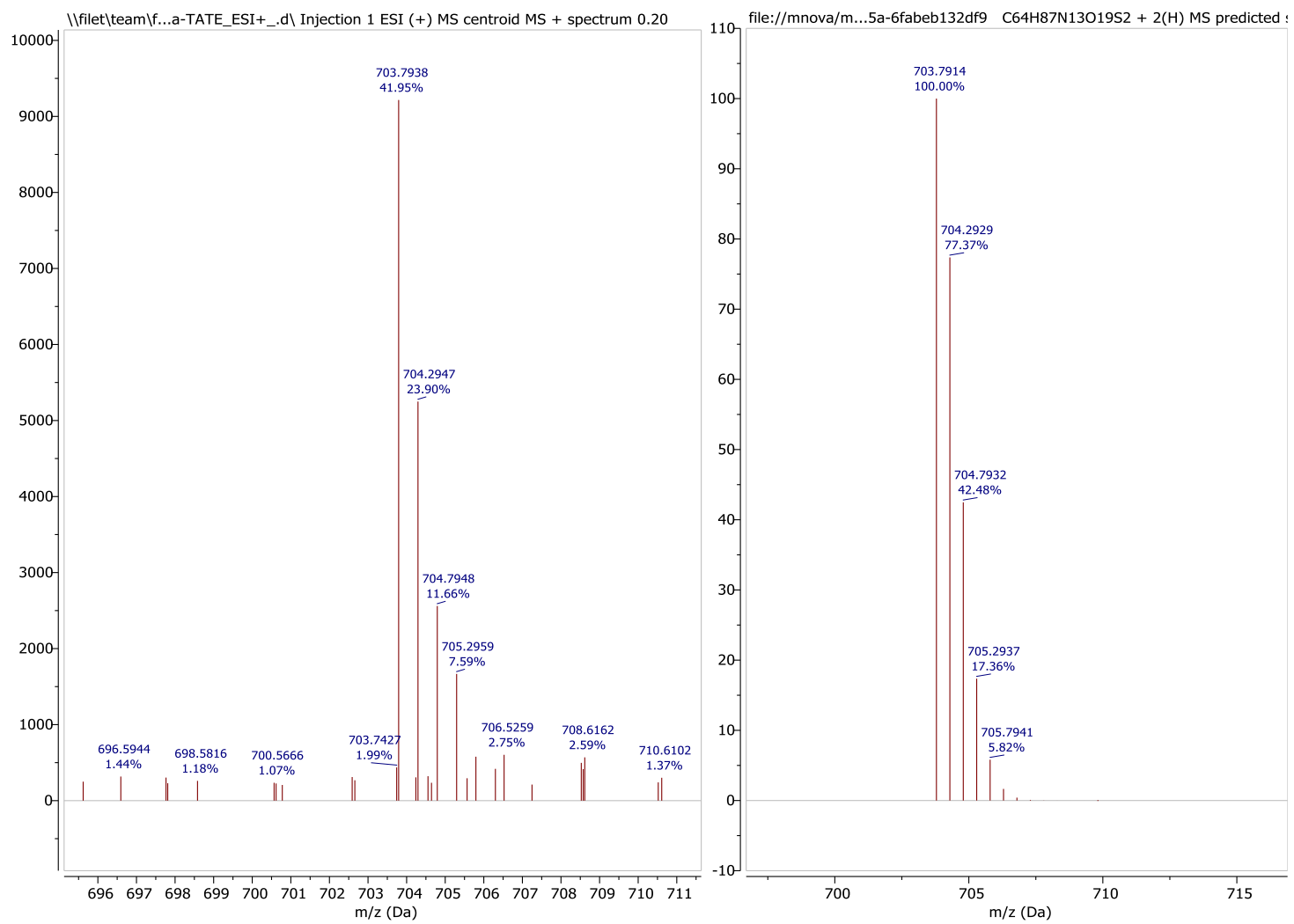


\section{NODAGA-Pra-O2Oc-TATE (5)}

Peptide 5 (28 mg, 23\%, white solid) was synthesized starting from Fmoc-Thr(tBu)-Wang resin according to GPs III and V (repetitive cycles), followed by GPs VIIIc and XV.

HPLC-Spectrum

H_25-75_5iso_25min_1mL_42total

mAU

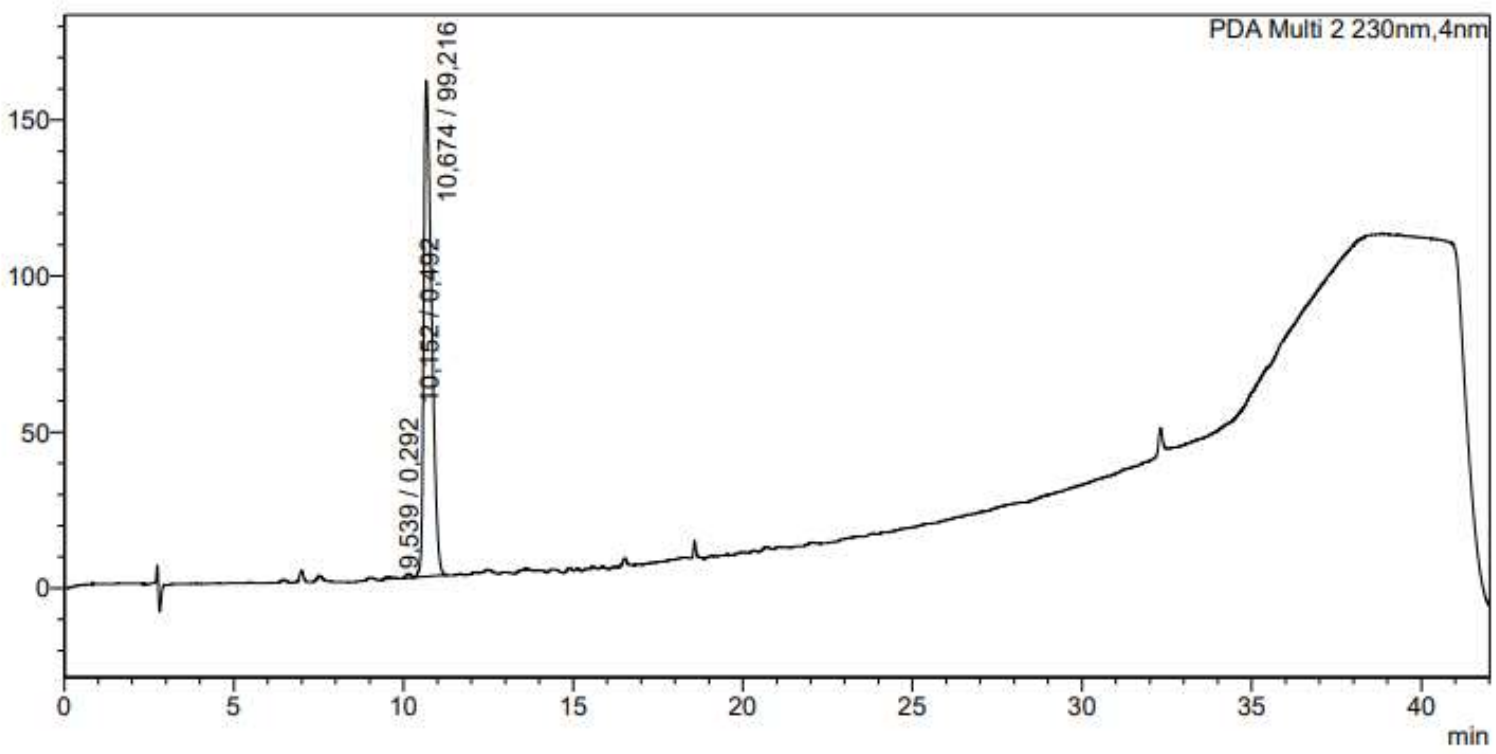

\begin{tabular}{rrrrrrr}
\multicolumn{2}{l}{ PDA Ch2 230nm } & \multicolumn{1}{l}{ Unit } & \multicolumn{1}{c}{ Area $\%$} \\
Peak\# & Ret. Time & \multicolumn{1}{c}{ Area } & Height & Conc. & Unit \\
1 & 9,539 & 7486 & 679 & 0,000 & 0,292 \\
2 & 10,152 & 12612 & 1339 & 0,000 & 0,492 \\
3 & 10,674 & 2543776 & 158426 & 0,000 & 99,216 \\
\hline Total & & 2563873 & 160444 & & & 100,000
\end{tabular}




\section{HRMS spectrum}

Sections of recorded mass spectra around the signal of $[\mathrm{M}+2 \mathrm{H}]^{2+}$ are shown on the left side versus the predicted $[\mathrm{M}+2 \mathrm{H}]^{2+}$ signal on the right side. Prediction of mass spectra was performed using the software MestreNova (version 14.2.1-27684).
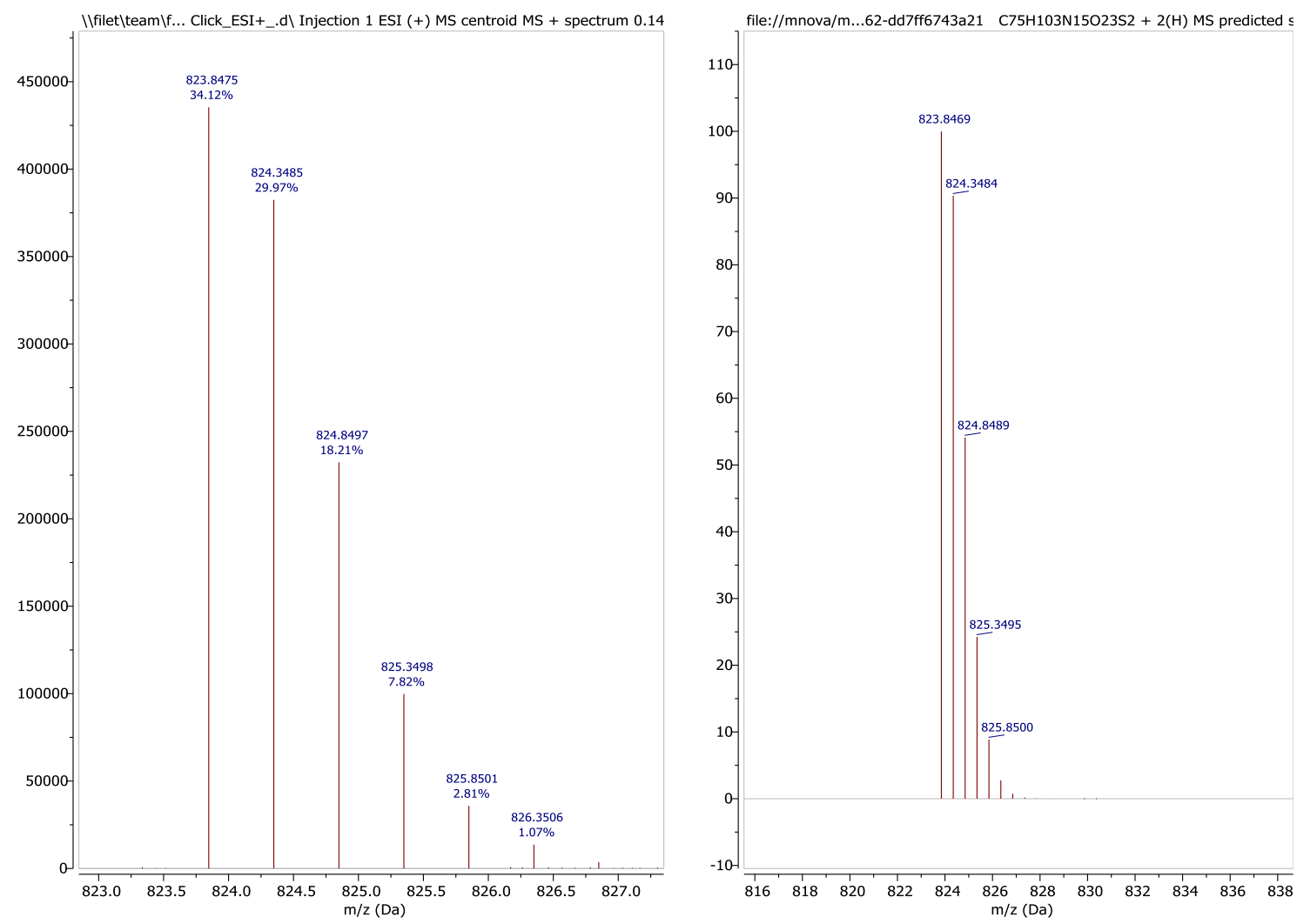


\section{NODAGA-CLAB1-TATE}

NODAGA-cLAB1-TATE (31 mg, 19\%, white solid) was synthesized starting from Fmoc$\operatorname{Thr}(t \mathrm{Bu})$-Wang resin according to GPs III and V (repetitive cycles), followed by GPs IX, VIIIc and XV.

HPLC-Spectrum

H_25-75_5iso_25min_1mL_42total

mAU

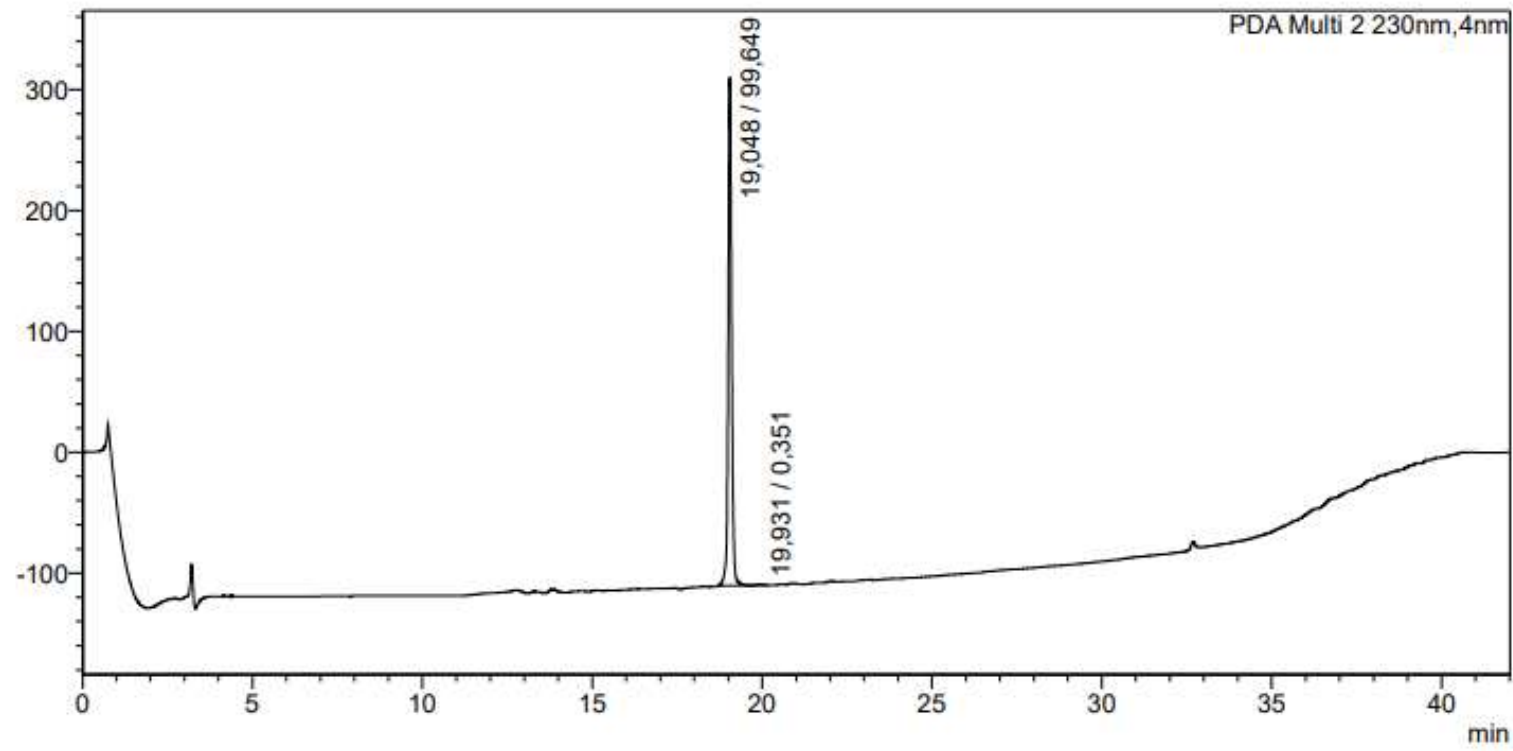

PDA Ch2 230nm

\begin{tabular}{|c|c|c|c|c|c|c|}
\hline Peak\# & Ret. Time & Area & Height & Conc. & Unit & Area\% \\
\hline 1 & 19,048 & 3000958 & 419463 & 0,000 & & 99,649 \\
\hline 2 & 19,931 & 10572 & 927 & 0,000 & & 0,351 \\
\hline Total & & 3011530 & 420390 & & & 100,000 \\
\hline
\end{tabular}




\section{HRMS spectrum}

Sections of recorded mass spectra around the signal of $[\mathrm{M}+2 \mathrm{H}]^{2+}$ are shown on the left side versus the predicted $[\mathrm{M}+2 \mathrm{H}]^{2+}$ signal on the right side. Prediction of mass spectra was performed using the software MestreNova (version 14.2.1-27684).
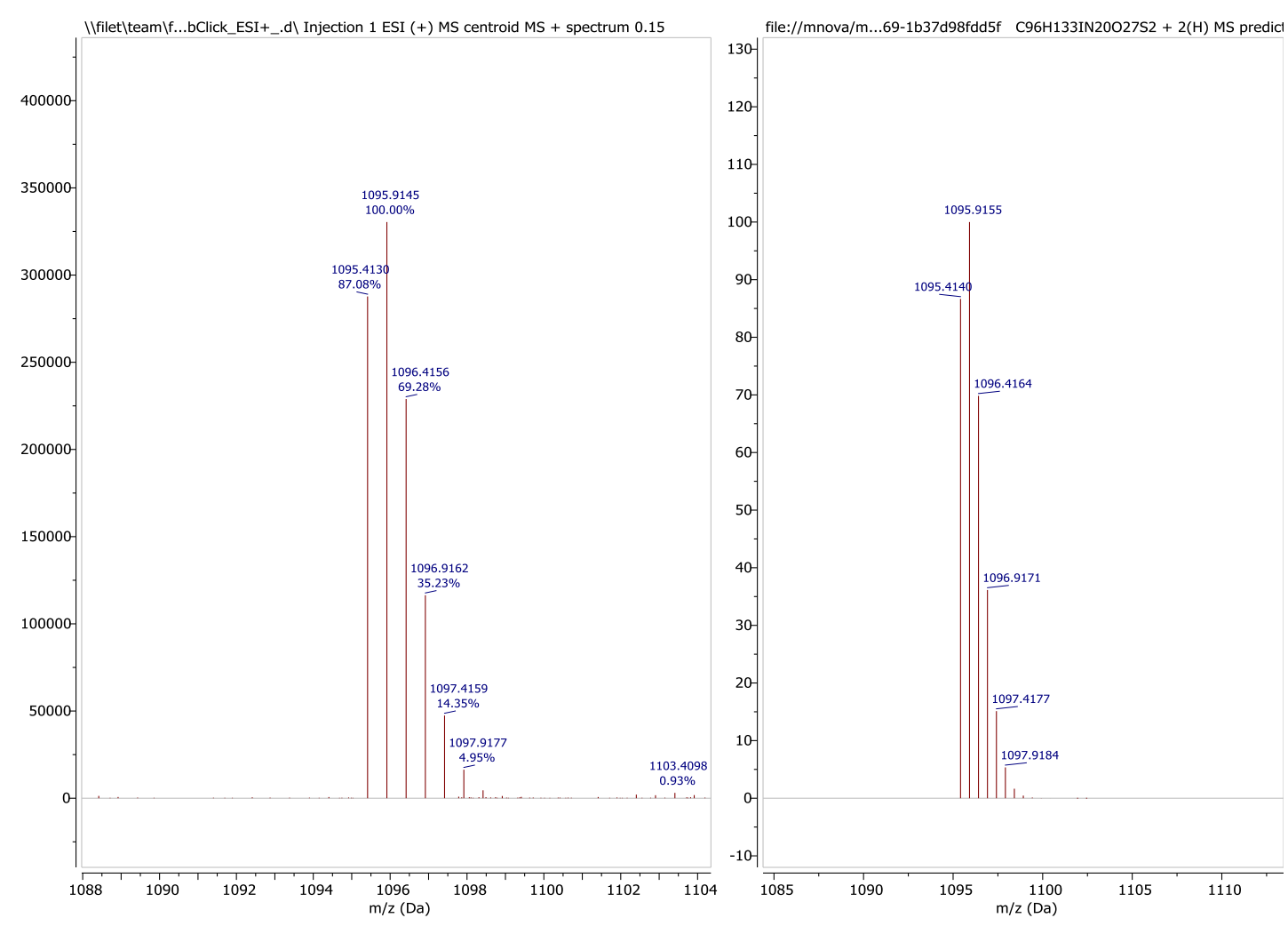


\section{NODAGA-cLAB2-TATE}

NODAGA-cLAB2-TATE (3 mg, 4\%, white solid) was synthesized starting from Fmoc-Thr(tBu)Wang resin according to GPs III and V (repetitive cycles), followed by GPs IX, VIIIc and XV. HPLC-Spectrum

H_25-75_5iso_25min_1mL_42total

mAU

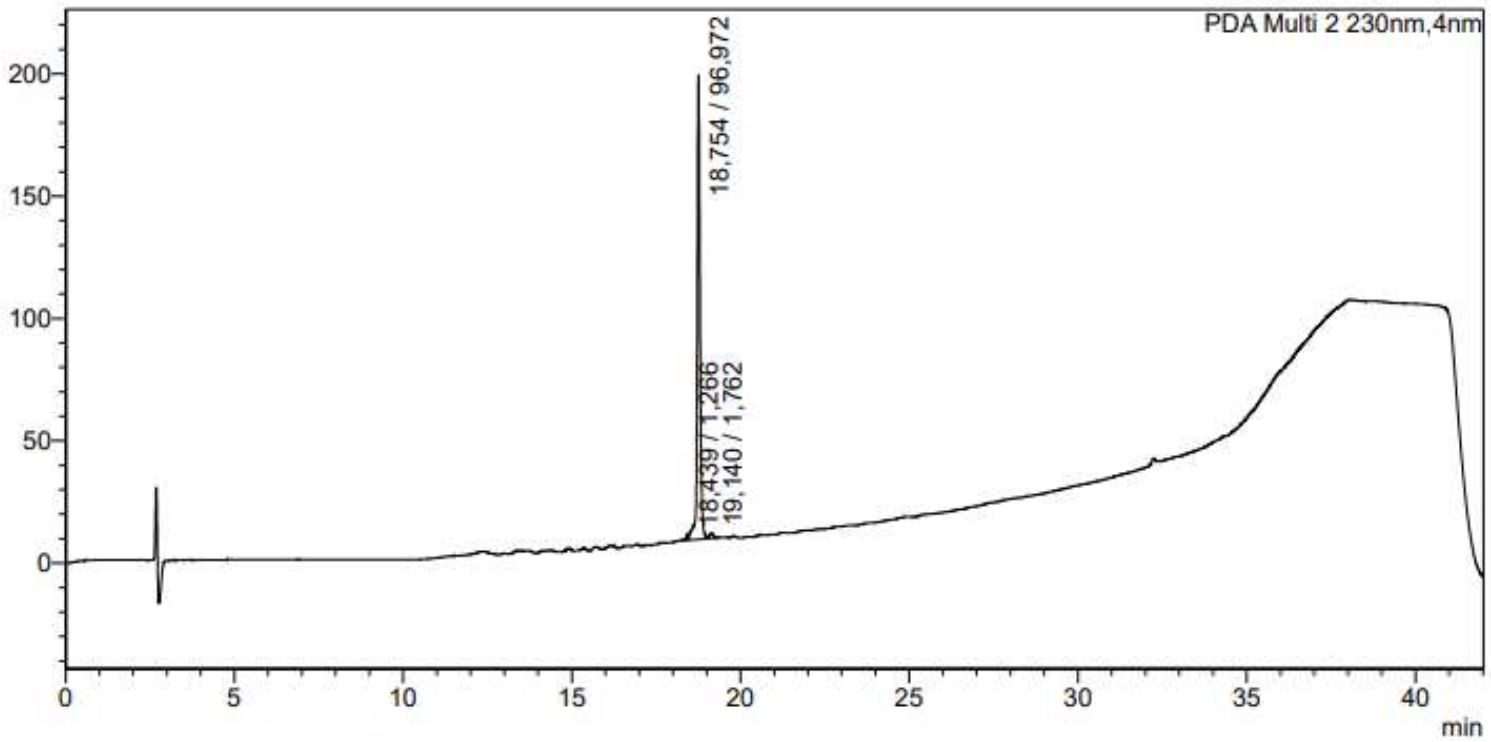

\begin{tabular}{|c|c|c|c|c|c|c|}
\hline \multicolumn{7}{|c|}{ PDA Ch2 230nm } \\
\hline Peak\# & Ret. Time & Area & Height & Conc. & Unit & Area $\%$ \\
\hline 1 & 18,439 & 14237 & 2463 & 0,000 & & 1,266 \\
\hline 2 & 18,754 & 1090361 & 188184 & 0,000 & & 96,972 \\
\hline 3 & 19,140 & 19807 & 1994 & 0,000 & & 1,762 \\
\hline Total & & 1124405 & 192641 & & & 100,000 \\
\hline
\end{tabular}




\section{HRMS spectrum}

Sections of recorded mass spectra around the signal of $[\mathrm{M}+2 \mathrm{H}]^{2+}$ are shown on the left side versus the predicted $[\mathrm{M}+2 \mathrm{H}]^{2+}$ signal on the right side. Prediction of mass spectra was performed using the software MestreNova (version 14.2.1-27684).
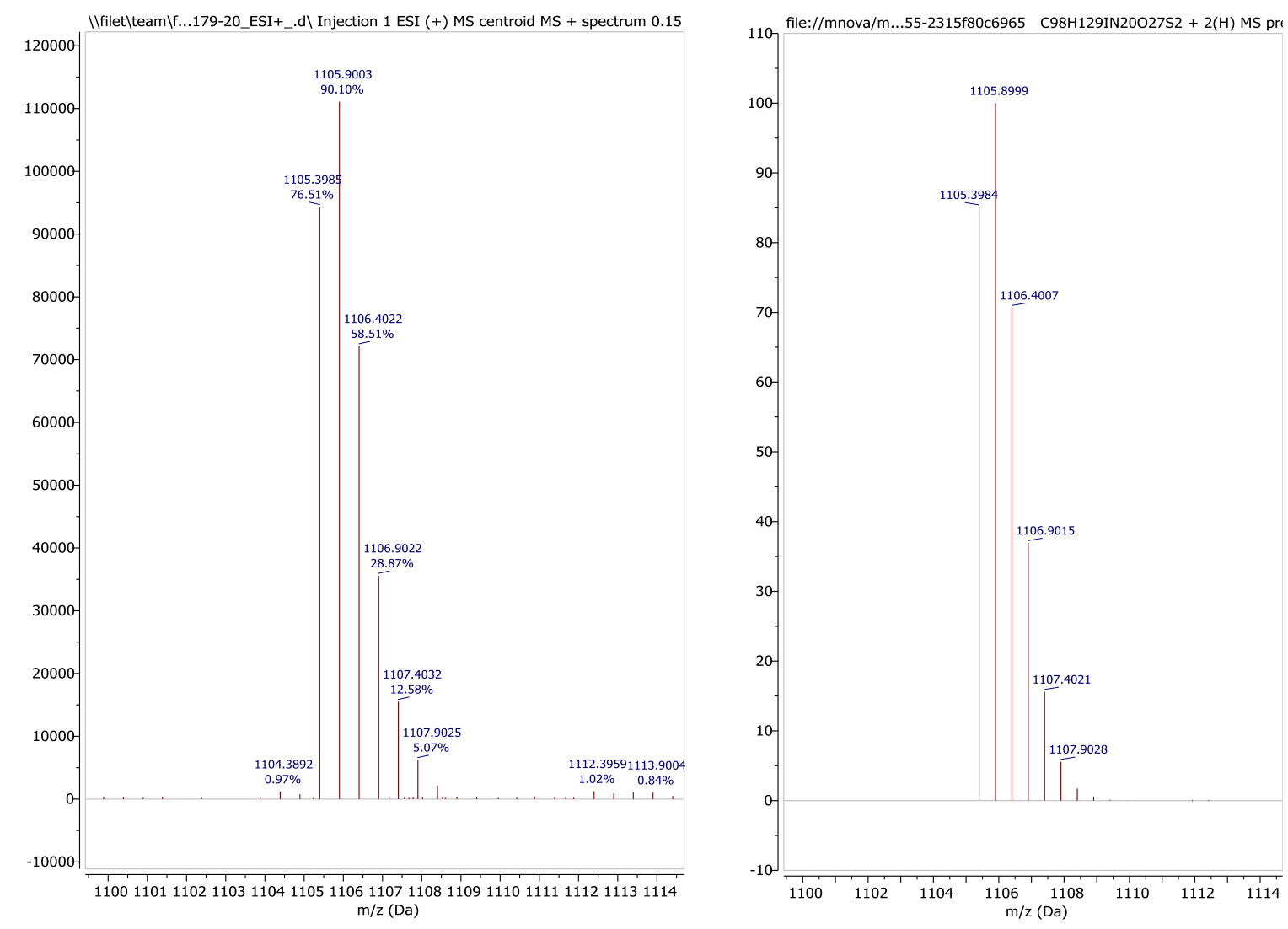


\section{NODAGA-cLAB3-TATE}

NODAGA-cLAB3-TATE (6 mg, 8\%, white solid) was synthesized starting from Fmoc-Thr(tBu)Wang resin according to GPs III and V (repetitive cycles), followed by GPs IX, VIIIc and XV. HPLC-Spectrum

H_25-75_5iso_25min_1mL_42total

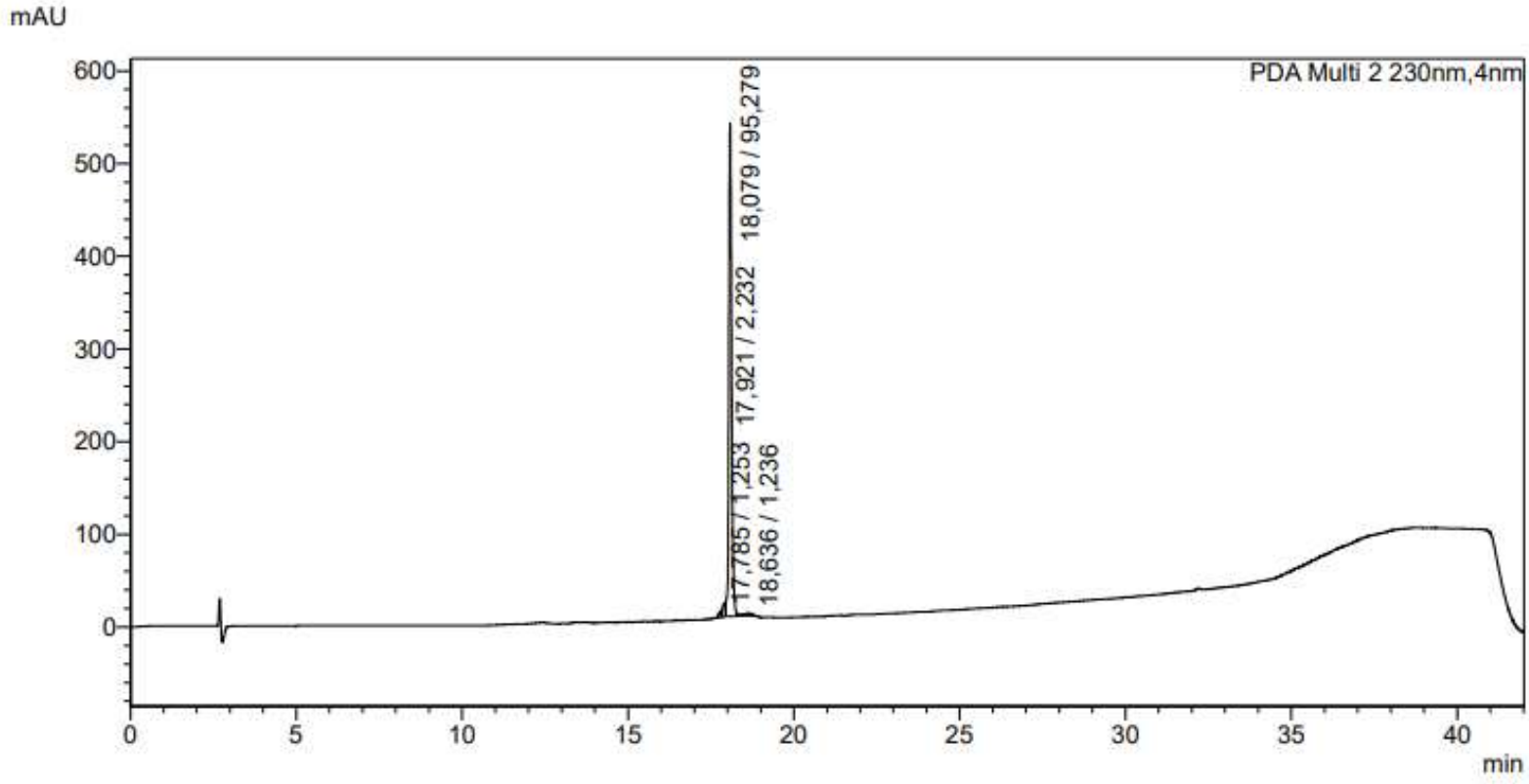

\begin{tabular}{|c|c|c|c|c|c|c|}
\hline & & & & & & \\
\hline Peak\# & Ret. Time & Area & Height & Conc. & Unit & Area $\%$ \\
\hline 1 & 17,785 & 39394 & 6687 & 0,000 & & 1,253 \\
\hline 2 & 17,921 & 70151 & 13892 & 0,000 & & 2,232 \\
\hline 3 & 18,079 & 2995071 & 530089 & 0,000 & & 95,279 \\
\hline 4 & 18,636 & 38857 & 3060 & 0,000 & & 1,236 \\
\hline Tota & & 3143472 & 553728 & & & 100,000 \\
\hline
\end{tabular}




\section{HRMS spectrum}

Sections of recorded mass spectra around the signal of $[\mathrm{M}+2 \mathrm{H}]^{2+}$ are shown on the left side versus the predicted $[\mathrm{M}+2 \mathrm{H}]^{2+}$ signal on the right side. Prediction of mass spectra was performed using the software MestreNova (version 14.2.1-27684).
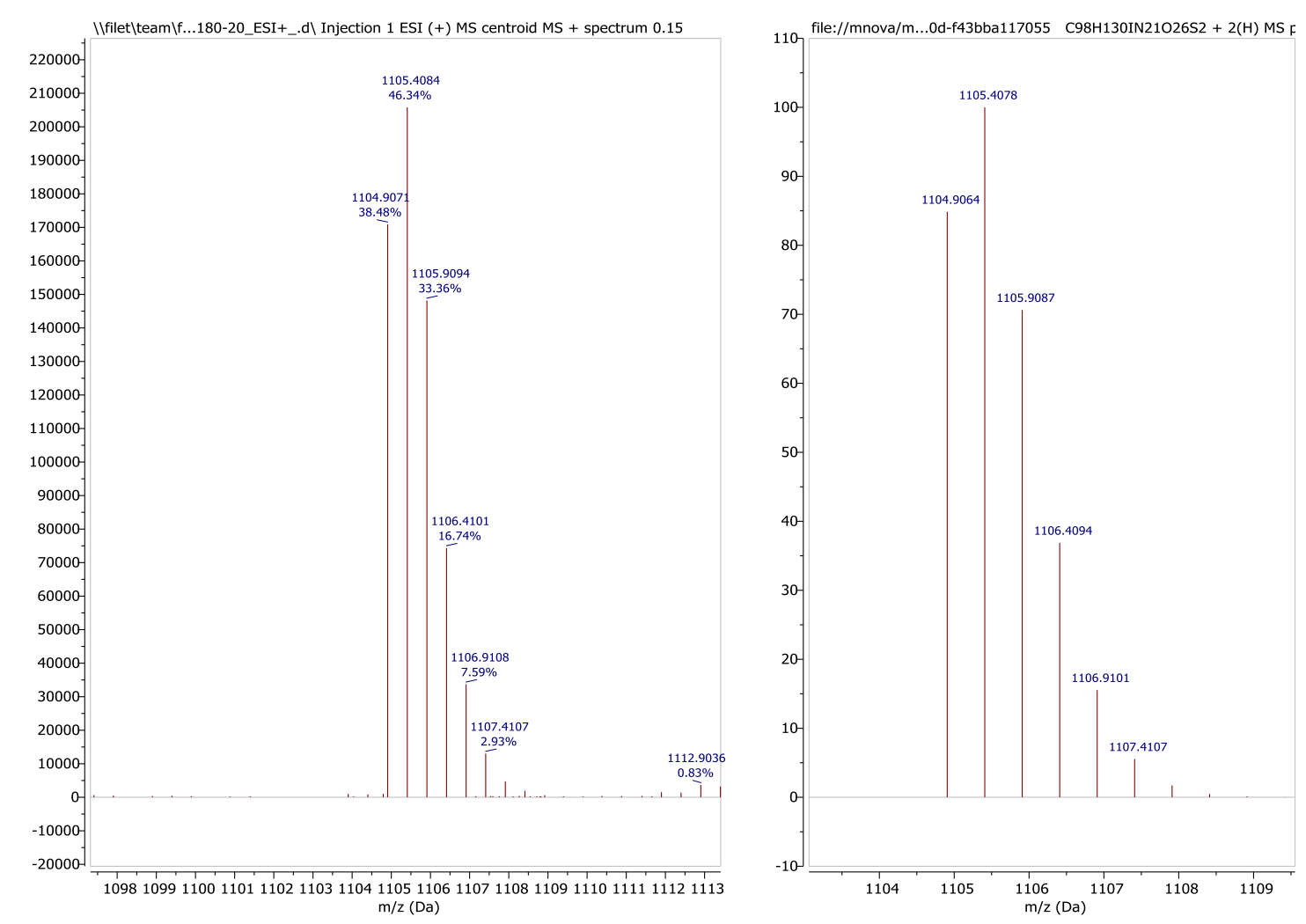


\section{NODAGA-cLAB4-TATE}

NODAGA-cLAB4-TATE (2.2 mg, 3\%, white solid) was synthesized starting from Fmoc$\operatorname{Thr}(t \mathrm{Bu})$-Wang resin according to GPs III and V (repetitive cycles), followed by GPs IX, VIIIc and XV.

HPLC-Spectrum

H_25-75_5iso_25min_1mL_42total

mAU

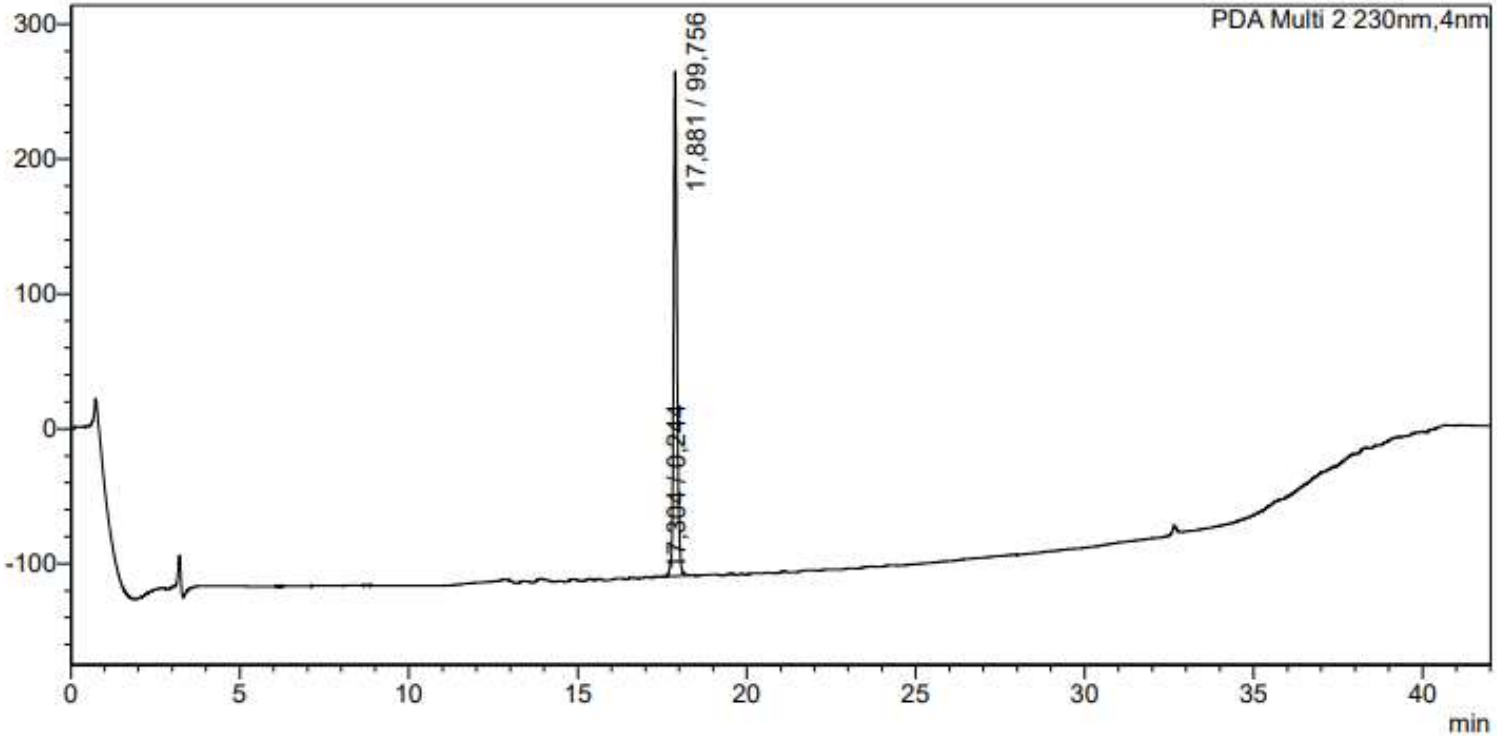

\begin{tabular}{|c|c|c|c|c|c|c|}
\hline \multicolumn{7}{|c|}{ PDA Ch2 230nm } \\
\hline Peak\# & Ret. Time & Area & Height & Conc. & Unit & Area $\%$ \\
\hline 1 & 17,304 & 6394 & 725 & 0,000 & & 0,244 \\
\hline 2 & 17,881 & 2612265 & 372385 & 0,000 & & 99,756 \\
\hline Total & & 2618660 & 373110 & & & 100,000 \\
\hline
\end{tabular}




\section{HRMS spectrum}

Sections of recorded mass spectra around the signal of $[\mathrm{M}+2 \mathrm{H}]^{2+}$ are shown on the left side versus the predicted $[\mathrm{M}+2 \mathrm{H}]^{2+}$ signal on the right side. Prediction of mass spectra was performed using the software MestreNova (version 14.2.1-27684).

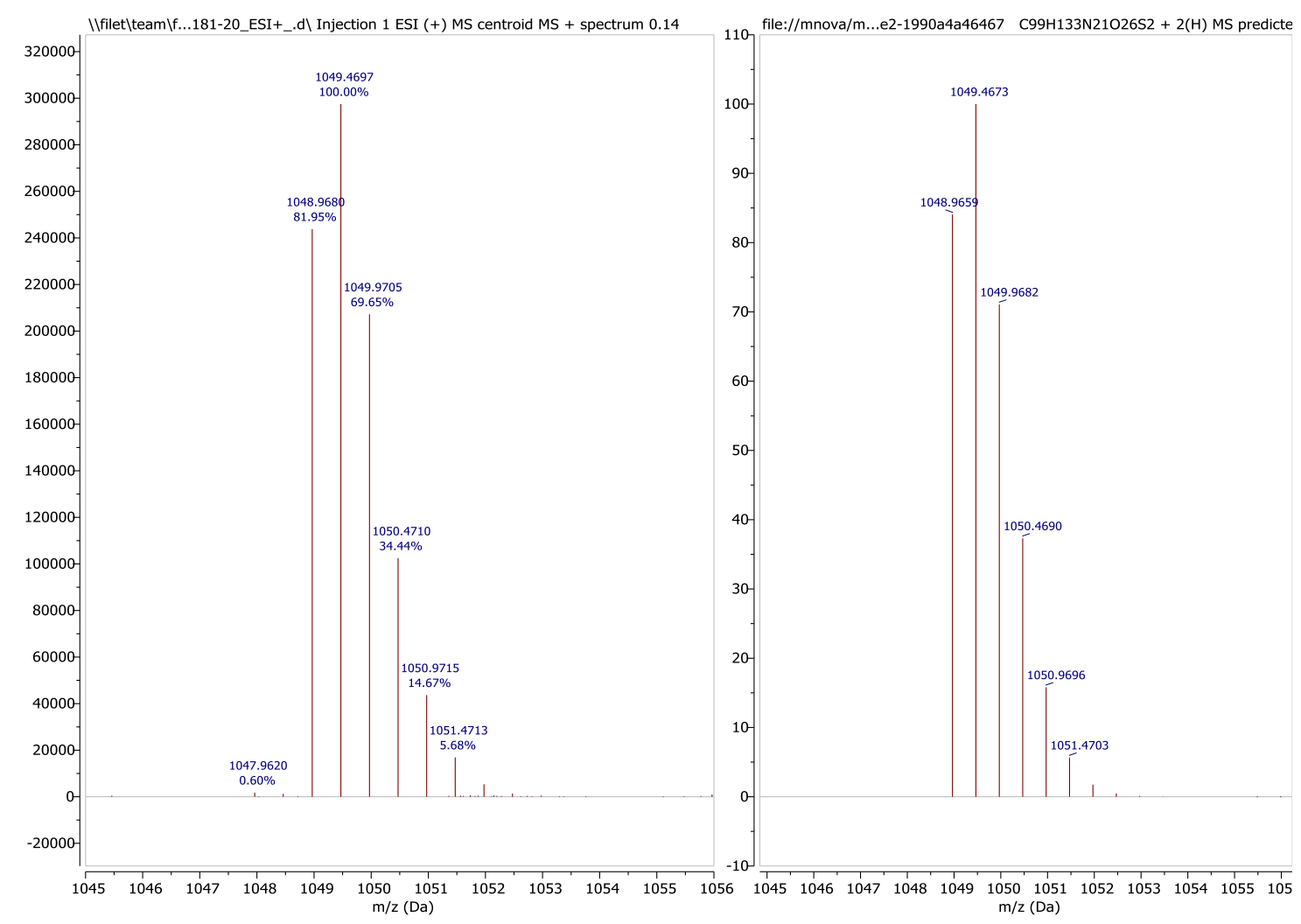


${ }^{64} \mathrm{Cu}-$-labeled TATE derivatives and the isolated albumin binder (S)-10

Exemplary radioactivity-detected chromatograms are shown below after labeling of the different TATE derivatives with copper-64. Labeling yields and purity were $>97 \%$.

\section{$\left[{ }^{64} \mathrm{Cu}\right] \mathrm{Cu}$-DOTA-TATE}

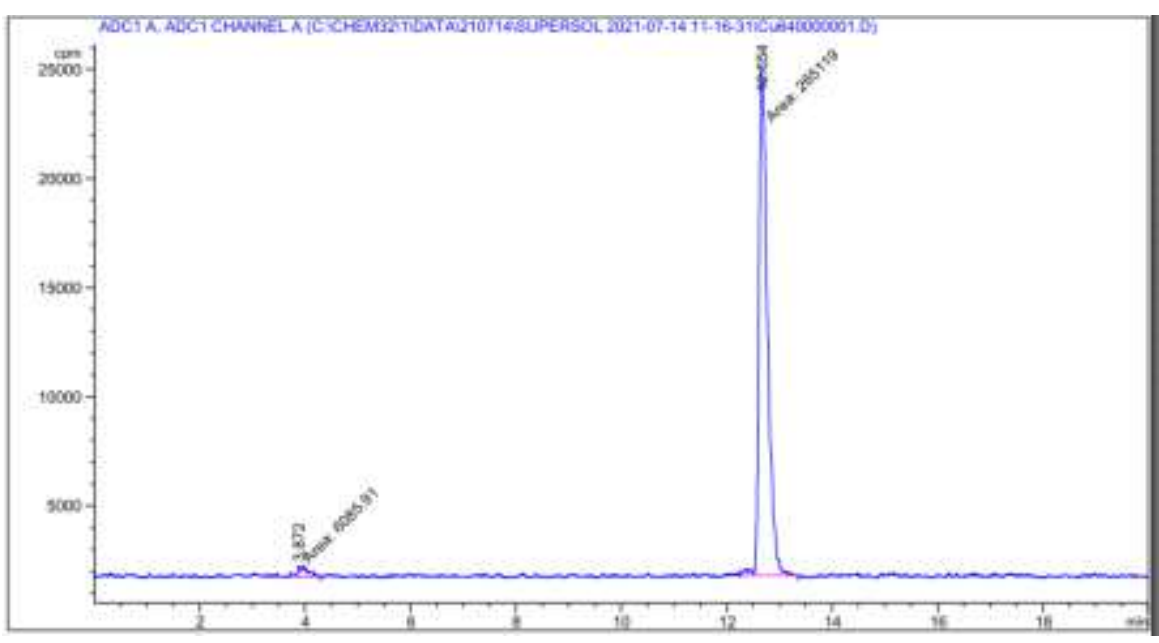

\begin{tabular}{|c|c|c|c|c|c|c|}
\hline $\begin{array}{c}\text { Peak } \\
\quad \#\end{array}$ & $\begin{array}{l}\text { RetTime } \\
\text { [min] }\end{array}$ & Type & $\begin{array}{l}\text { Width } \\
\text { [min] }\end{array}$ & $\begin{array}{c}\text { Area } \\
{\left[\mathrm{cpm}^{*} \mathrm{~s}\right]}\end{array}$ & $\begin{array}{l}\text { Height } \\
\text { [cpm] }\end{array}$ & $\begin{array}{c}\text { Area } \\
\quad 8\end{array}$ \\
\hline 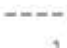 & $-\infty$ & Me & ( ) 2000 & & & \\
\hline 2 & $\begin{array}{r}3.872 \\
12.654\end{array}$ & $\begin{array}{l}M M \\
M M\end{array}$ & $\begin{array}{l}0.2099 \\
0.1904\end{array}$ & $\begin{array}{l}6085.91260 \\
2.65119 e 5\end{array}$ & $\begin{array}{l}483.16196 \\
2.32061 \mathrm{e}\end{array}$ & $\begin{array}{r}2.2440 \\
97.7560\end{array}$ \\
\hline
\end{tabular}

\section{$\left[{ }^{64} \mathrm{Cu}\right] \mathrm{Cu}-\mathrm{NODAGA-TATE}$}

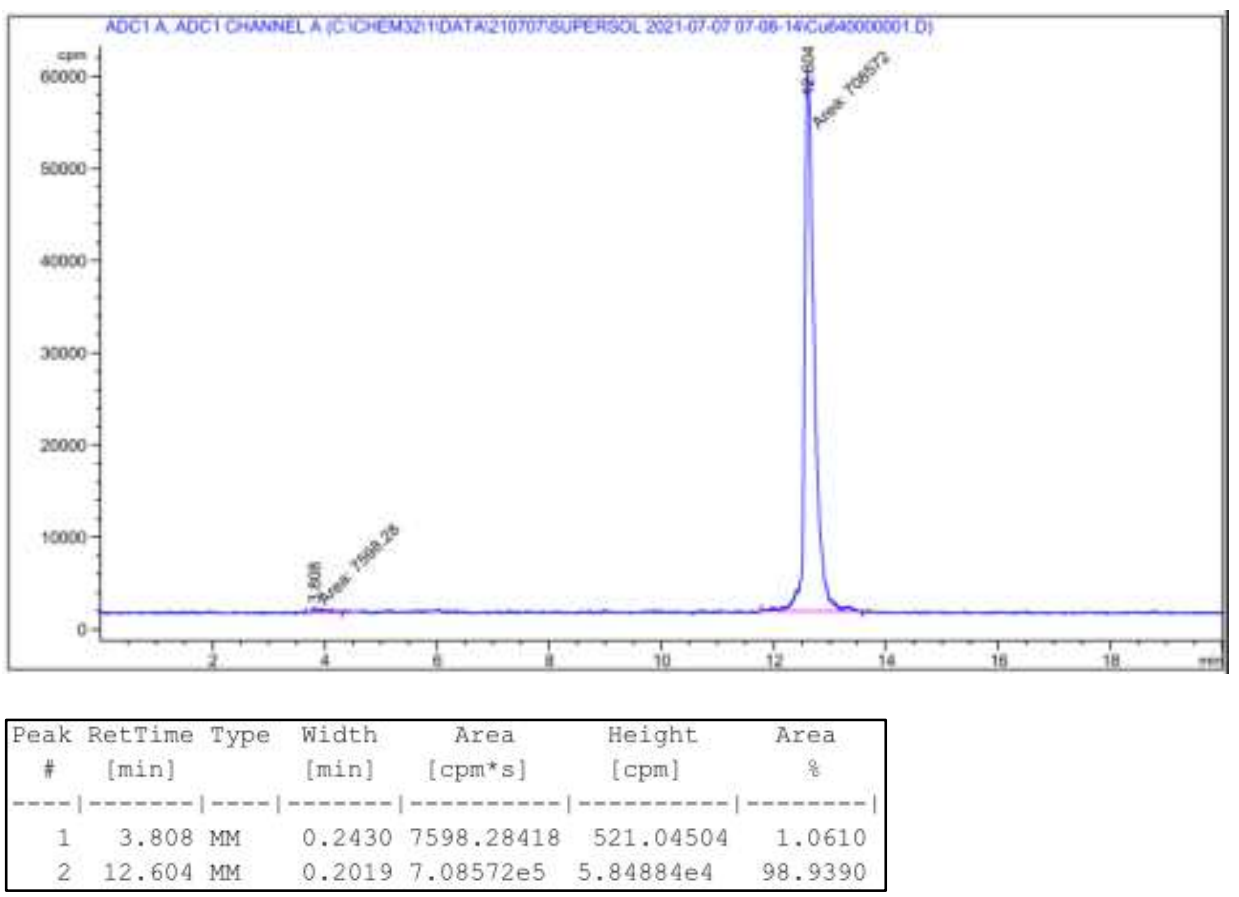




\section{$\left[{ }^{64} \mathrm{Cu}\right] \mathrm{Cu}-5$}

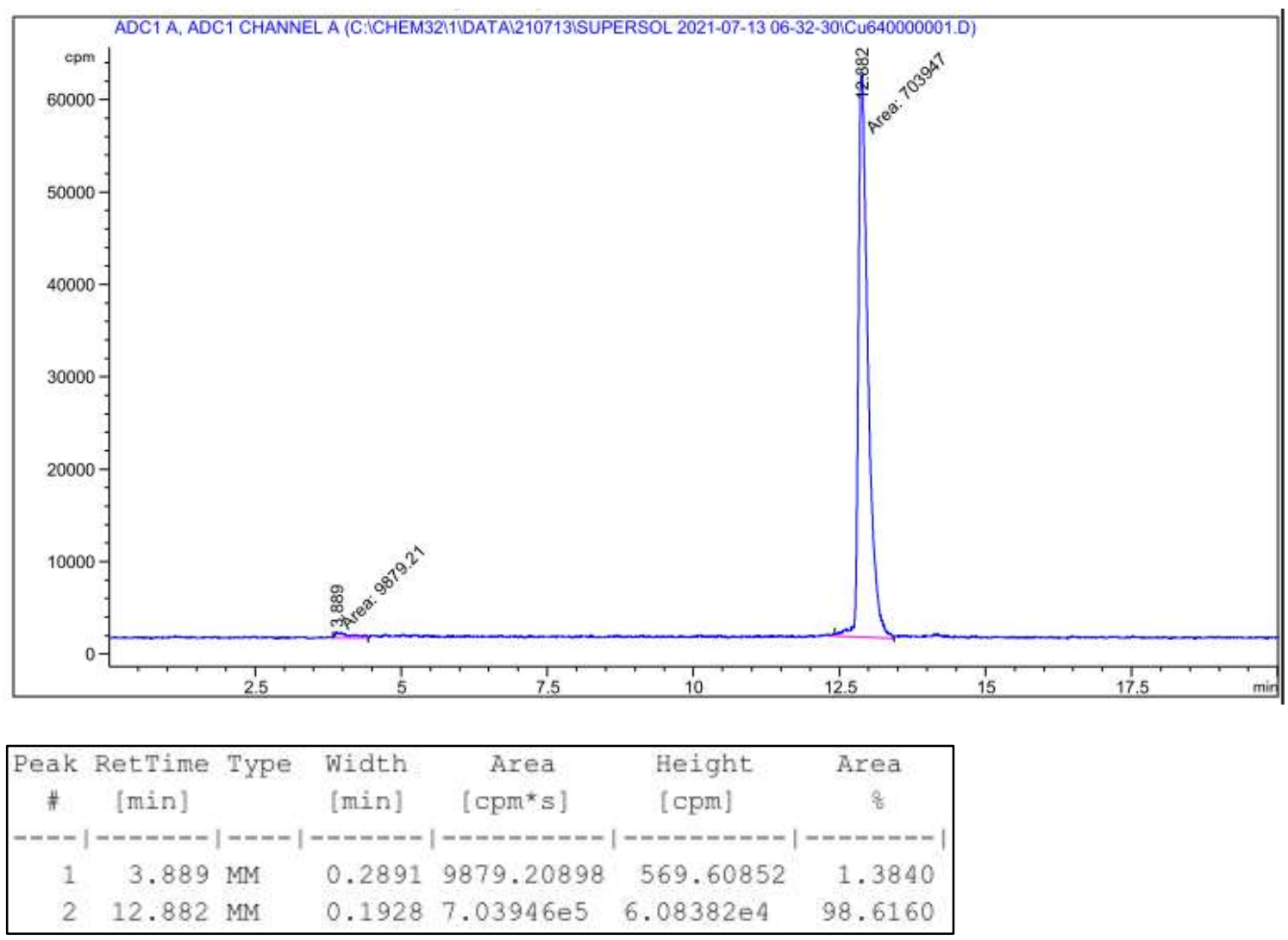

\section{$\left[{ }^{64} \mathrm{Cu}\right] \mathrm{Cu}-N O D A G A-c L A B 1-T A T E$}

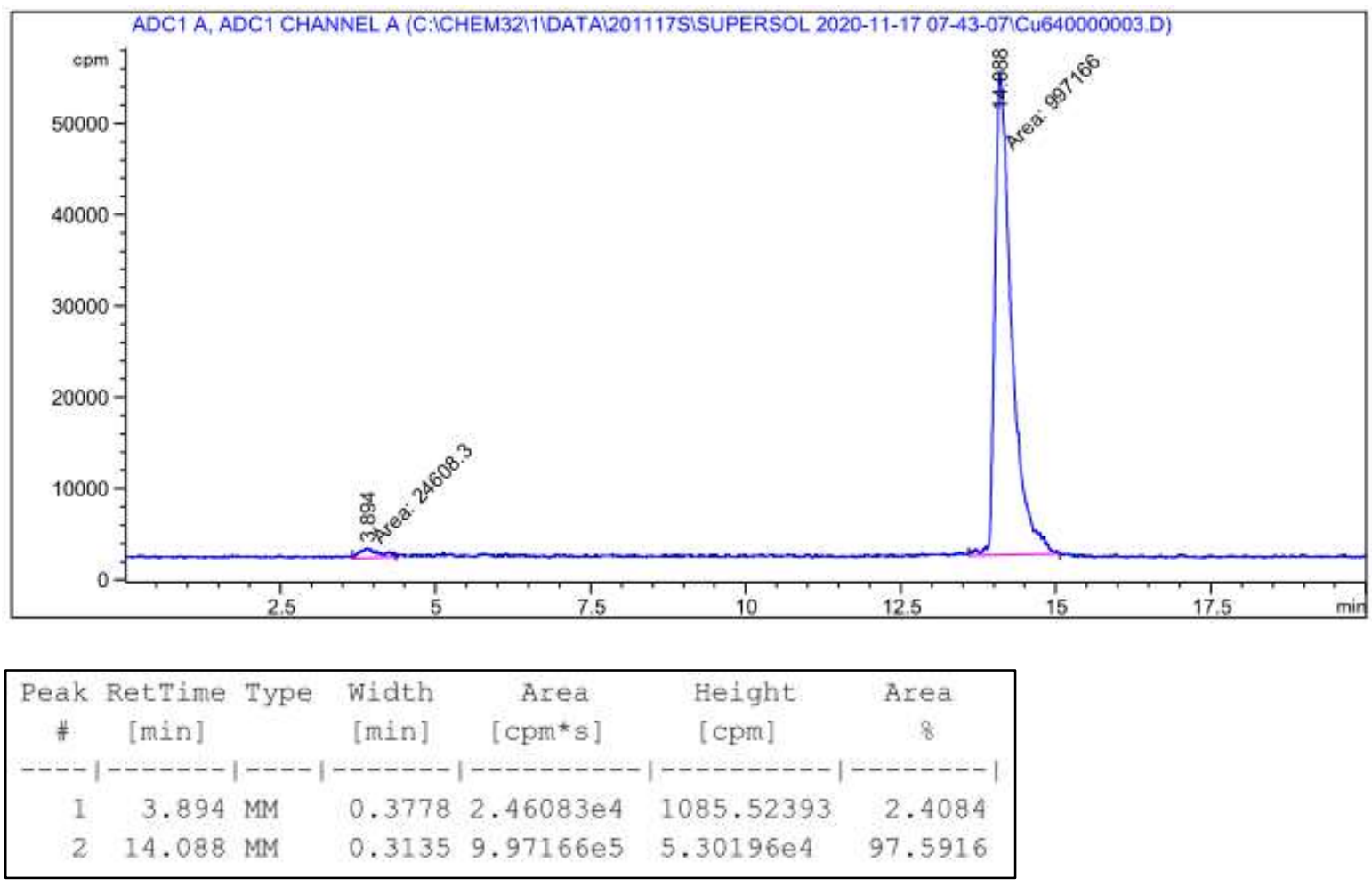




\section{$\left[{ }^{64} \mathrm{Cu}\right] \mathrm{Cu}-N O D A G A-c L A B 2-T A T E$}

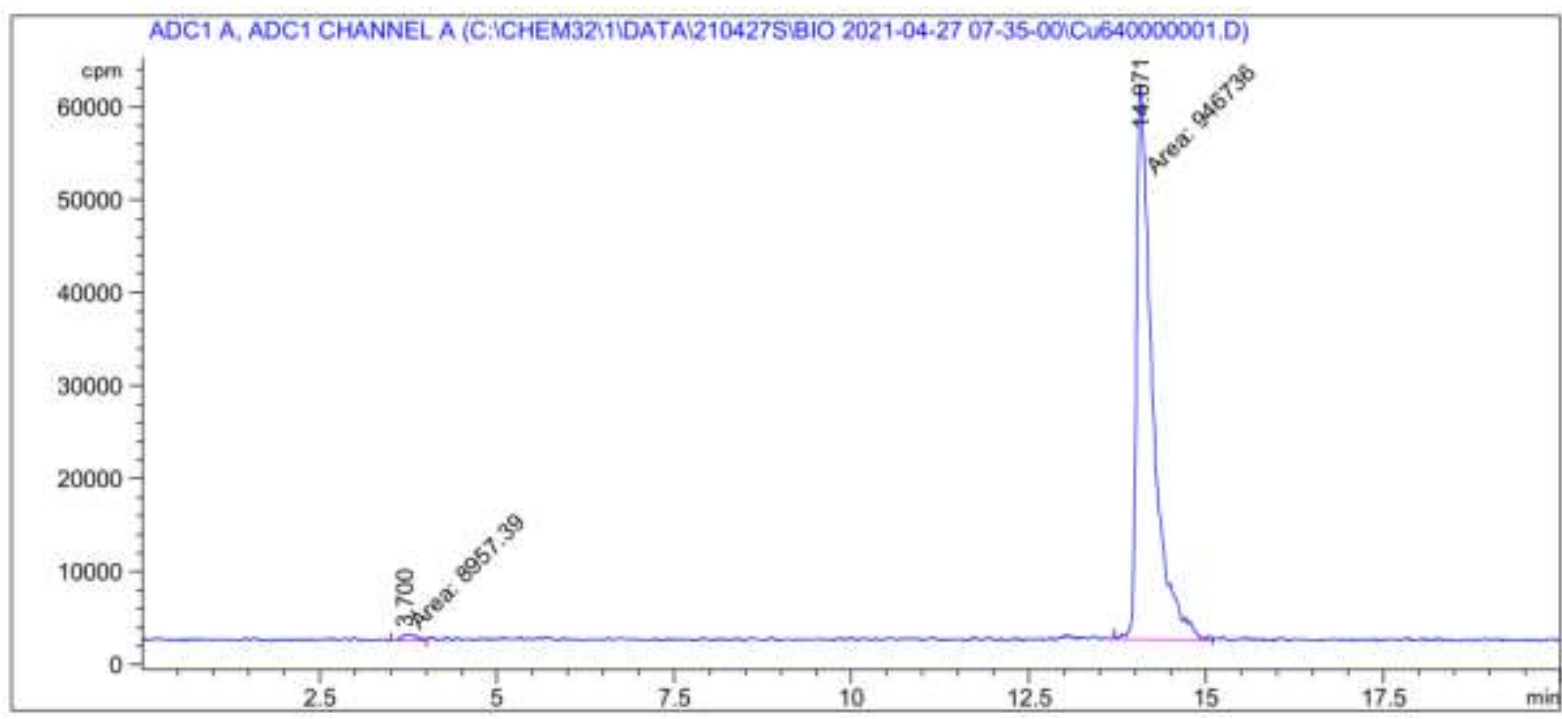

\begin{tabular}{|c|c|c|c|c|c|c|}
\hline $\begin{array}{c}\text { Peak } \\
\#\end{array}$ & $\begin{array}{c}\text { RetTime } \\
\text { [min] }\end{array}$ & Type & $\begin{array}{l}\text { Width } \\
\text { [min] }\end{array}$ & $\begin{array}{c}\text { Area } \\
{\left[\mathrm{cpm}^{\star} \mathrm{s}\right]}\end{array}$ & $\begin{array}{l}\text { Height } \\
{[\mathrm{cpm}]}\end{array}$ & $\begin{array}{c}\text { Area } \\
\text { 음 }\end{array}$ \\
\hline 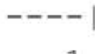 & & & ก: 0100 & $00-7000-0$ & $-1-0-1--1$ & $-\cdots$ \\
\hline 1 & 3.700 & MM & 0.2192 & 8957.39453 & 680.95319 & 0.9373 \\
\hline 2 & 14.071 & MM & 0.2632 & $9.46736 \mathrm{e} 5$ & $5.99486 e 4$ & 99.0627 \\
\hline
\end{tabular}

\section{$\left[{ }^{64} \mathrm{Cu}\right] \mathrm{Cu}-\mathrm{NODAGA}-\mathrm{CLAB3}-\mathrm{TATE}$}

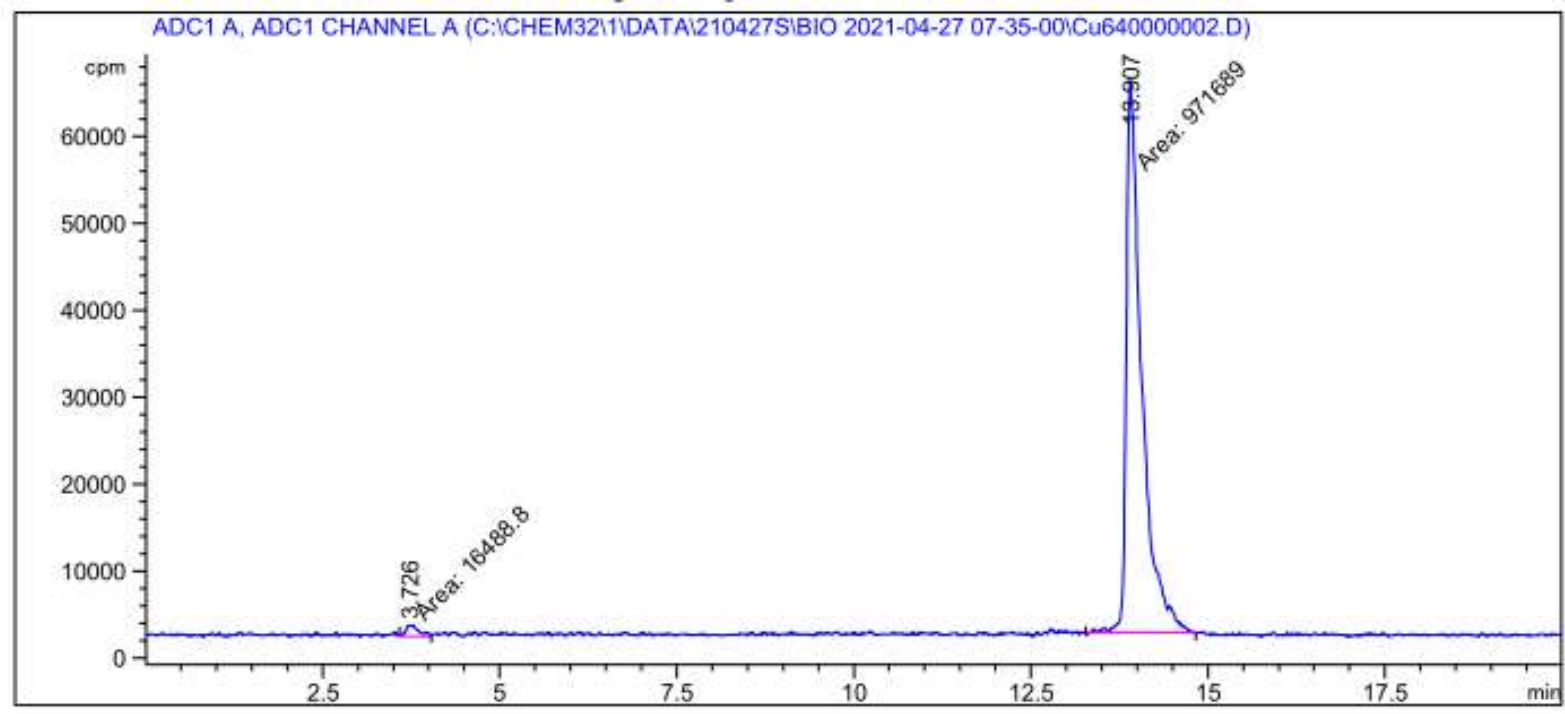

\begin{tabular}{|c|c|c|c|c|c|c|}
\hline $\begin{array}{c}\text { Peak } \\
\#\end{array}$ & $\begin{array}{c}\text { RetTime } \\
\text { [min] }\end{array}$ & Type & $\begin{array}{l}\text { Width } \\
\text { [min] }\end{array}$ & $\begin{array}{c}\text { Area } \\
{\left[\mathrm{cpm}^{\star} \mathrm{s}\right]}\end{array}$ & $\begin{array}{l}\text { Height } \\
\text { [cpm] }\end{array}$ & $\begin{array}{c}\text { Area } \\
\&\end{array}$ \\
\hline & & & 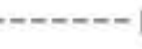 & - & 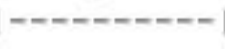 & $--\infty-\infty$ \\
\hline 1 & 3.726 & MM & 0.2114 & $1.64888 \mathrm{e} 4$ & 1299.83105 & 1.6686 \\
\hline 2 & 13.907 & $\mathrm{MM}$ & 0.2550 & $9.71689 e 5$ & $6.35134 \mathrm{e} 4$ & 98.3314 \\
\hline
\end{tabular}




\section{$\left[{ }^{64} \mathrm{Cu}\right] \mathrm{Cu}-N O D A G A-c L A B 4-T A T E$}

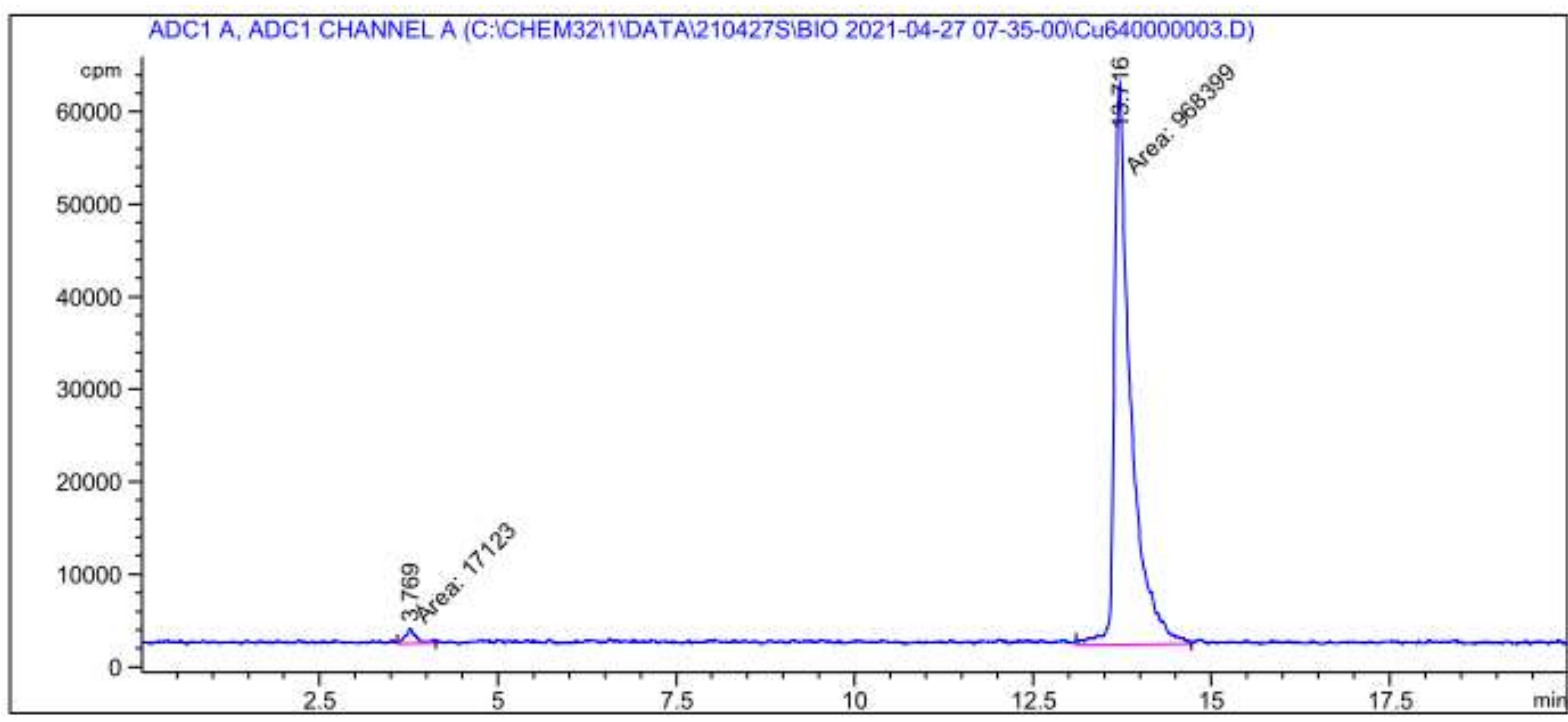

\begin{tabular}{|cccccc|}
\hline $\begin{array}{c}\text { Peak RetTime Type } \\
\text { \# } \\
\text { [min] }\end{array}$ & $\begin{array}{c}\text { Width } \\
\text { [min] }\end{array}$ & $\begin{array}{c}\text { Area } \\
\text { [cpm*s] }\end{array}$ & $\begin{array}{c}\text { Height } \\
\text { [cpm] }\end{array}$ & $\begin{array}{c}\text { Area } \\
\&\end{array}$ \\
\hdashline 1 & $3.769 \mathrm{MM}$ & 0.1717 & $1.71230 \mathrm{e} 4$ & 1662.56006 & 1.7375 \\
2 & $13.716 \mathrm{MM}$ & 0.2663 & $9.68399 \mathrm{e} 5$ & $6.05974 \mathrm{e} 4$ & 98.2625 \\
\hline
\end{tabular}

\section{$\left[{ }^{64} \mathrm{Cu}\right] \mathrm{Cu}-(S)-10$}

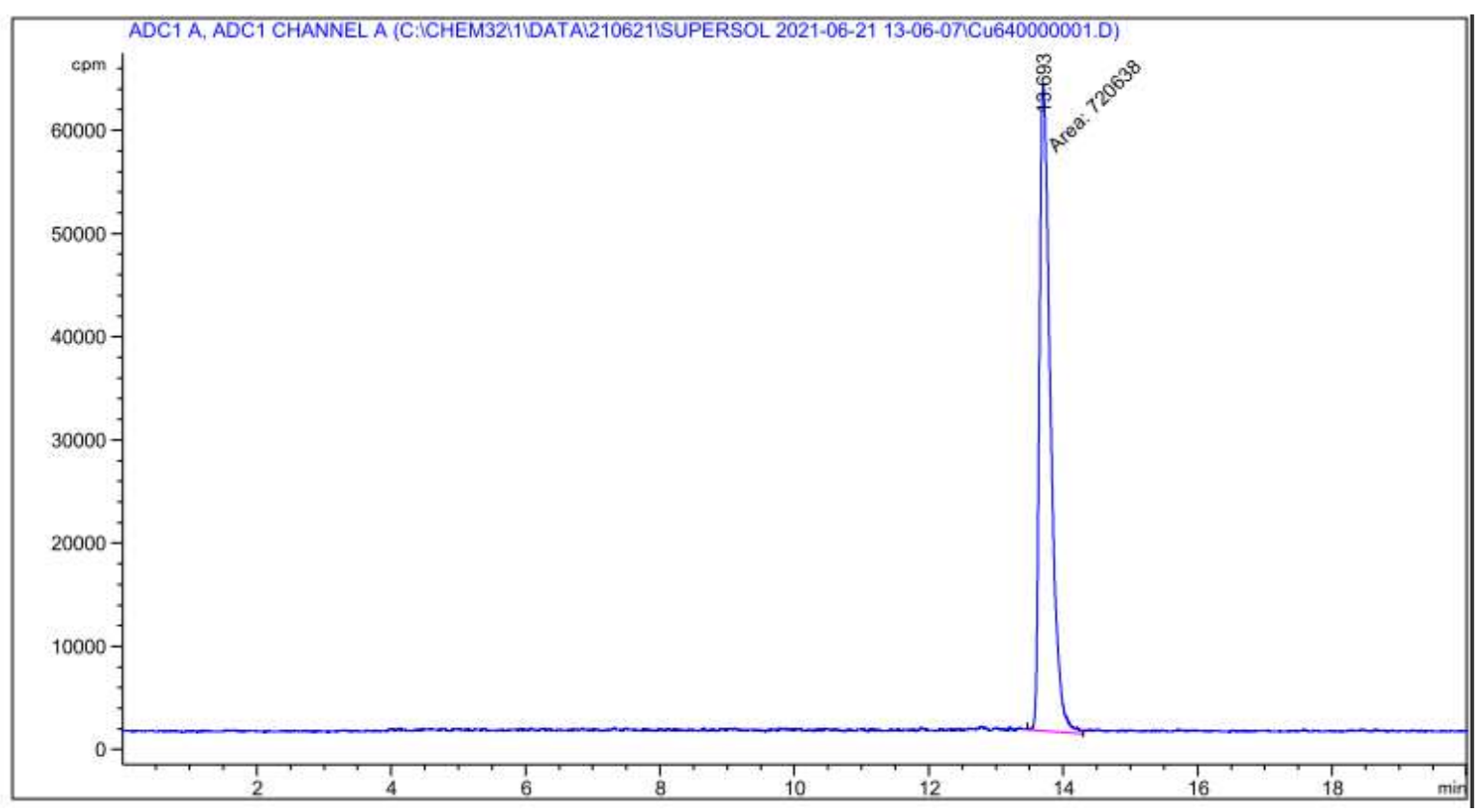

\begin{tabular}{|c|c|c|c|c|c|c|}
\hline $\begin{array}{c}\text { Peak } \\
\#\end{array}$ & $\begin{array}{l}\text { RetTime } \\
\text { [min] }\end{array}$ & Type & $\begin{array}{l}\text { Width } \\
\text { [min] }\end{array}$ & $\begin{array}{c}\text { Area } \\
{\left[\mathrm{cpm}^{\star} s\right]}\end{array}$ & $\begin{array}{l}\text { Height } \\
\text { [cpm] }\end{array}$ & $\begin{array}{c}\text { Area } \\
\text { \& }\end{array}$ \\
\hline 1 & 13.693 & MM & 0.1919 & $7.20638 \mathrm{e} 5$ & $6.25744 \mathrm{e}$ & 100.0000 \\
\hline
\end{tabular}




\section{References}

1. Farrera-Sinfreu, J.; Royo, M. and Albericio, F. Undesired removal of the Fmoc group by the free $\varepsilon$-amino function of a lysine residue. Tetrahedron Lett., 2002, 43, 7813-7815.

2. Wilson, K. R.; Sedberry, S.; Pescatore, R.; Vinton, D.; Love, B.; Ballard, S.; Wham, B. C.; Hutchison, S. K. and Williamson, E. J. Microwave-assisted cleavage of Alloc and Allyl Ester protecting groups in solid phase peptide synthesis. J. Pept. Sci., 2016, 22, 622-627.

3. Using microwave heating to expedite your allyl ester or alloc deprotection. https://selekt.biotage.com/peptideblogs/using-microwave-heating-to-expedite-your-allyl-ester-or-allocdeprotection.

4. Dumelin, C. E.; Trussel, S.; Buller, F.; Trachsel, E.; Bootz, F.; Zhang, Y.; Mannocci, L.; Beck, S. C.; DrumeaMirancea, M.; Seeliger, M. W.; Baltes, C.; Muggler, T.; Kranz, F.; Rudin, M.; Melkko, S.; Scheuermann, J. and Neri, D. A portable albumin binder from a DNA-encoded chemical library. Angew. Chem. Int. Ed. Engl., 2008, 47, 3196-3201.

5. Henczi, M. and Weaver, D. F. An Improved Synthesis of Ne-Fmoc-L-Lysine and Nס-Fmoc-L-Ornithine. OPPI Briefs, 1994, 26, 578-580.

6. Hulme, E. C. and Trevethick, M. A. Ligand binding assays at equilibrium: validation and interpretation. Br. J. Pharmacol., 2010, 161, 1219-1237.

7. Tummino, P. J. and Copeland, R. A. Residence time of receptor-ligand complexes and its effect on biological function. Biochemistry, 2008, 47, 5481-5492.

8. Reubi, J. C.; Schär, J. C.; Waser, B.; Wenger, S.; Heppeler, A.; Schmitt, J. S. and Mäcke, H. R. Affinity profiles for human somatostatin receptor subtypes SST1-SST5 of somatostatin radiotracers selected for scintigraphic and radiotherapeutic use. Eur. J. Nucl. Med., 2000, 27, 273-282.

9. Zeggari, M.; Viguerie, N.; Susini, C.; Esteve, J. P.; Vaysse, N.; Rivier, J.; Wunsch, E. and Ribet, A. Characterization of pancreatic somatostatin binding sites with a ${ }^{125}$ I-somatostatin 28 analog. Peptides, 1986, 7, 953-959.

10. Hansch, C. and Leo, A., Exploring QSAR Fundamentals and Applications in Chemistry and Biology, American Chemical Society, Washington, 1995.

11. Hansch, C.; Leo, A. and Hoekman, D., Exploring QSAR: Hydrophobic, Electronic, and Steric Constants, American Chemical Society, USA, 1995.

12. Druckrey, E. Regressionsanalyse von Struktur-Wirkungs-Beziehungen (Hansch-Analyse). Pharm. unserer Zeit, 1975, 4, 145-150.

13. Kubinyi, H., QSAR: Hansch Analysis and Related Approaches, VCH, Weinheim, 1993.

14. Benesova, M.; Umbricht, C. A.; Schibli, R. and Müller, C. Albumin-Binding PSMA Ligands: Optimization of the Tissue Distribution Profile. Mol. Pharm., 2018, 15, 934-946.

15. Fischer, R.; Mader, O.; Jung, G. and Brock, R. Extending the applicability of carboxyfluorescein in solid-phase synthesis. Bioconjug. Chem., 2003, 14, 653-660.

16. Kvach, M. V.; Stepanova, I. A.; Prokhorenko, I. A.; Stupak, A. P.; Bolibrukh, D. A.; Korshun, V. A. and Shmanai, V. V. Practical synthesis of isomerically pure 5- and 6-carboxytetramethylrhodamines, useful dyes for DNA probes. Bioconjug. Chem., 2009, 20, 1673-1682.

17. Qin, L.; Hu, B.; Neumann, K. D.; Linstad, E. J.; McCauley, K.; Veness, J.; Kempinger, J. J. and DiMagno, S. G. A Mild and General One-Pot Synthesis of Densely Functionalized Diaryliodonium Salts. Eur. J. Org. Chem., 2015, 2015, 5919-5924.

18. Fiala, T.; Wang, J.; Dunn, M.; Sebej, P.; Choi, S. J.; Nwadibia, E. C.; Fialova, E.; Martinez, D. M.; Cheetham, C. E.; Fogle, K. J.; Palladino, M. J.; Freyberg, Z.; Sulzer, D. and Sames, D. Chemical Targeting of Voltage Sensitive Dyes to Specific Cells and Molecules in the Brain. J. Am. Chem. Soc., 2020, 142, 9285-9301.

19. Kotali, A.; Tanaka, M.; Hirata, Y.; Sawaguchi, T. and Kurosawa, S. Synthesis and structures of zwitterionic polymers to induce electrostatic interaction with PDMS surface treated by air-plasma. Arkivoc, 2018, 2018, 330-343.

20. Eichenberger, L. S.; Patra, M. and Holland, J. P. Photoactive chelates for radiolabelling proteins. Chem. Commun., 2019, 55, 2257-2260. 